\title{
Characterization of Modern Ammunition and Background Profiles: A Novel Approach and Probabilistic Interpretation of Inorganic Gunshot Residue
}

\author{
Korina Layli Menking-Hoggatt \\ West Virginia University, klmenkinghoggatt@mail.wvu.edu
}

Follow this and additional works at: https://researchrepository.wvu.edu/etd

Part of the Analytical Chemistry Commons, Inorganic Chemistry Commons, Materials Chemistry

Commons, Multivariate Analysis Commons, and the Probability Commons

\footnotetext{
Recommended Citation

Menking-Hoggatt, Korina Layli, "Characterization of Modern Ammunition and Background Profiles: A Novel Approach and Probabilistic Interpretation of Inorganic Gunshot Residue" (2021). Graduate Theses, Dissertations, and Problem Reports. 8336.

https://researchrepository.wvu.edu/etd/8336

This Dissertation is protected by copyright and/or related rights. It has been brought to you by the The Research Repository @ WVU with permission from the rights-holder(s). You are free to use this Dissertation in any way that is permitted by the copyright and related rights legislation that applies to your use. For other uses you must obtain permission from the rights-holder(s) directly, unless additional rights are indicated by a Creative Commons license in the record and/ or on the work itself. This Dissertation has been accepted for inclusion in WVU Graduate Theses, Dissertations, and Problem Reports collection by an authorized administrator of The Research Repository @ WVU. For more information, please contact researchrepository@mail.wvu.edu.
} 


\title{
Characterization of Modern Ammunition and Background Profiles: A Novel Approach and Probabilistic Interpretation of Inorganic Gunshot Residue
}

\author{
Korina Menking-Hoggatt, M.S. \\ Dissertation submitted \\ to the Eberly College of Arts and Sciences \\ at West Virginia University \\ Doctor of Philosophy in \\ Forensic Science \\ Tatiana Trejos, Ph.D., Chair \\ Keith Morris, Ph.D. \\ Luis Arroyo, Ph.D. \\ Stacey Culp, Ph.D.
}

Department of Forensic and Investigative Science

\author{
Morgantown, West Virginia \\ 2021
}

Keywords: Scanning Electron Microscopy- Energy Dispersive X-ray Spectrometry (SEM-EDS),

Laser-Induced Breakdown Spectroscopy (LIBS), gunshot residue (GSR), rapid technology, validation, nontoxic ammunition

Copyright 2021 Korina Menking-Hoggatt 


\title{
Abstract \\ Characterization of Modern Ammunition and Background Profiles: A Novel Approach and Probabilistic Interpretation of Inorganic Gunshot Residue
}

\author{
Korina Menking-Hoggatt
}

Gun violence is a leading cause of premature death in the U.S., with 13,507 firearm-related deaths reported in the first quarter of 2021. ${ }^{1}$ The issue of gun violence is complex and includes unintentional shootings, defensive use, homicides, suicides, assault with a deadly weapon, and mass shootings. From a criminal justice perspective, the accurate reconstruction of events and prompt apprehension of individuals of interest is critical. During these investigations, gunshot residue (GSR) detection is essential and, therefore, a commonly submitted form of evidence to forensic laboratories. The standard method adopted at crime laboratories to detect inorganic gunshot residue (IGSR) is Scanning Electron Microscopy-Energy Dispersive X-ray Spectrometry (SEM-EDS). The main advantage of SEM-EDS technology is its capability for single particle analysis, including morphology and elemental composition. However, the SEM-EDS data acquisition process is time-consuming. Moreover, the emergence of new types of nontoxic ammunition is shifting the list of potential elements and moving away from the traditional lead, barium, and antimony elements.

As a result, the forensic community has recognized the need for complementary approaches to build knowledge on contemporary formulations and understand the implications in the evidence interpretation and, ultimately, its significance to the trier of fact. This research aims to fill some of these demands by developing innovative standard materials and alternative methods to characterize and interpret modern ammunition.

This study developed standards from discharged primer containing gunshot residue microparticles ranging from $0.5 \mu \mathrm{m}$ to $10 \mu \mathrm{m}$. Twenty standards with leaded and lead-free primer-GSR (pGSR) microparticles were created and characterized by three analytical techniques (SEM-EDS, Laser-Induced Breakdown Spectroscopy (LIBS), and Inductively Coupled Plasma- Mass Spectrometry (ICP-MS). The number of GSR particles, their composition, and elemental concentrations demonstrated stability for over a year, presenting an attractive resource to be used as ground truth for the quality control of GSR analysis, validation of existing and emerging methods, and interlaboratory testing. The pGSR standards showed some elemental profiles not yet reported in the literature, making available new relevant information to practitioners.

Also, this research conducted an extensive survey of low and high-risk background populations (i.e., professions and hobbies which may mimic the presence of GSR), along with samples from controlshooters who fired various firearms and primers. The authentic samples were analyzed by SEM-EDS and by a novel, alternative analysis proposed as a fast screening test (LIBS). A dataset was obtained from authentic samples from the hands of 975 individuals, providing an extensive characterization of over 2,900 samples. The comprehensive population study permitted testing different statistical methods for predictive classification, machine learning algorithms and probabilistic assessment of the evidential value of GSR, with power of the tests and accuracies generally better than $90 \%$ depending on the classifier applied.

This research provides the forensic science community with updated information of chemical composition of modern ammunition, assessment of current methods and a rapid screening method for GSR, and quantitative statistical interpretation of GSR evidence. The data generated in this study is anticipated to provide forensic examiners and the criminal justice with an essential leap of knowledge and the tools necessary to reach more definitive conclusions in the courtroom.

${ }^{1}$ GUN VIOLENCE Achieve http://www.gunviolencearchive.org/ (accessed Apr 19, 2021). 


\section{Acknowledgements}

My journey through my Ph.D. has been exciting, challenging, and an experience that I will carry with me throughout my life. As with any great accomplishment, I did not get through this alone. A great team of amazing individuals supported me through the good and the bad. There were times when I would hit a wall and become so overwhelmed, but they would not let me give up. They reminded me that I could get through this and were with me every set of the way At this time I would like to thank them all for their unwavering support.

I would like to acknowledge my committee chair, Dr. Tatiana Trejos, for her help and assistance through every step of this project. She has been a pillar of support and a wonderful mentor on my Ph.D. journey. She gave me every opportunity and resource to accomplish my project and professional goals. She has been a role model to look up to and emulate. Most of all, she has been a friend and confidant through the entire process, a shoulder to cry on and someone to share in my victories.

I would also like to thank my other committee members. Dr. Keith has been an invaluable member of the committee, teaching me all I know about firearms and criminalistics. Dr. Arroyo has provided insight into the analytical process and helped me to fine tune my analytical skills. Dr. Culp has assisted me with statistics I thought I would never understand and helped me become a more rounded scientist. All of them also provided guidance and support throughout my project.

I would also like to thank my research group family. All the members have helped me with data collection and analysis throughout this large population study. They shared the tears and victories, stayed by my side when research became challenging, and reminded me to walk away when needed. I never would have made it this far without them.

Finally, I would like to thank my personal support group of friends and family for always believing in me. Their words of support and encouragement remained with me through every challenge I have faced, even if they did not understand what I was doing. They reminded me that I was strong enough to accomplish this project and gave me their strength when I needed it the most. Their unwavering love and support kept me going.

Alone we can do so little; together we can do so much. - Helen Keller 


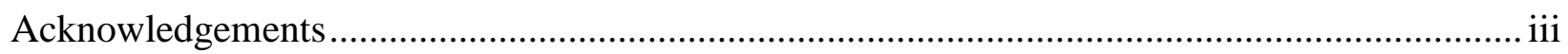

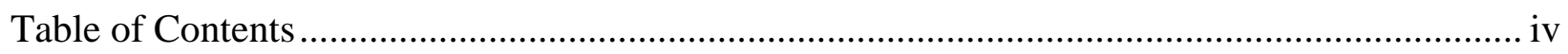

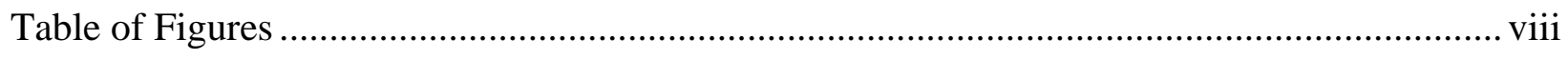

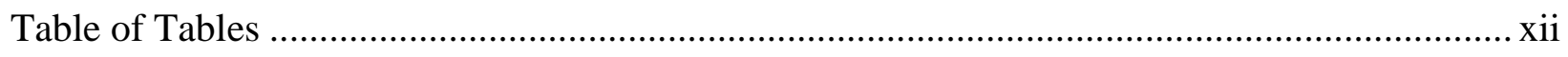

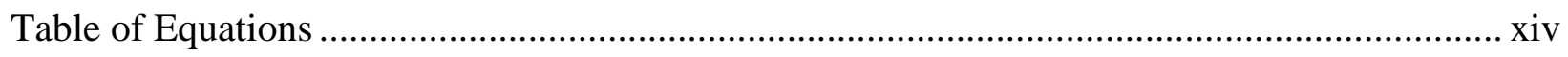

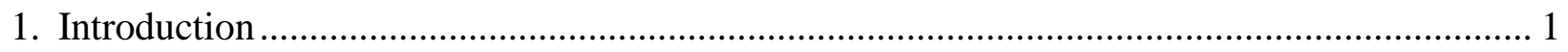

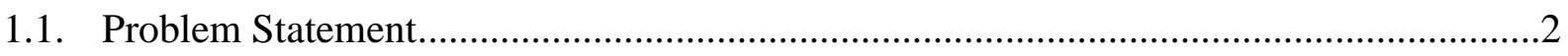

1.2. Composition, Morphology and Detection of Gunshot Residue .........................................3

1.3. Analytical Methods for Detection and Identification of GSR ........................................

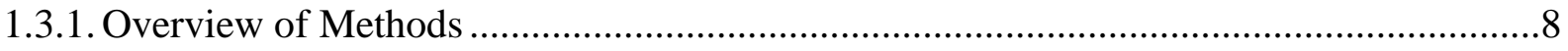

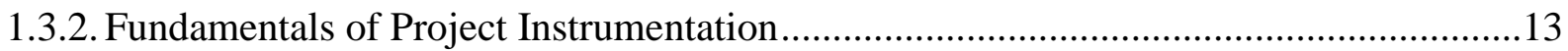

1.3.2.1. Inductively Coupled Plasma- Mass Spectrometry (ICP-MS) .................................13

1.3.2.2. Scanning Electron Microscopy- Energy Dispersive X-ray Spectroscopy (SEM-

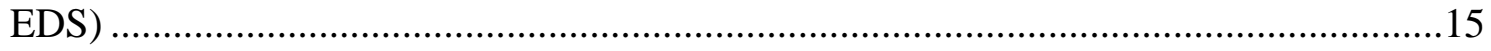

1.3.2.3. Laser Induced Breakdown Spectroscopy (LIBS) ………......................................16

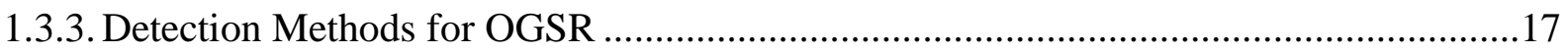

1.3.4. Simultaneous or Sequential Detection Methods for the Identification of OGSR and

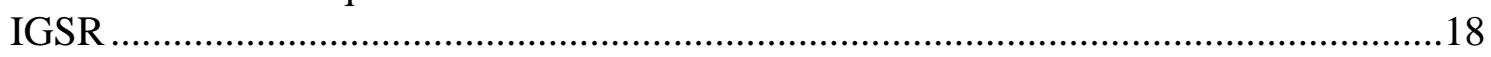

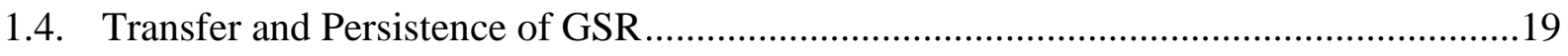

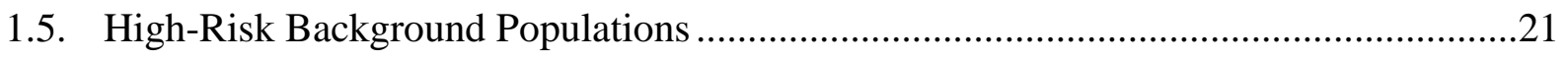

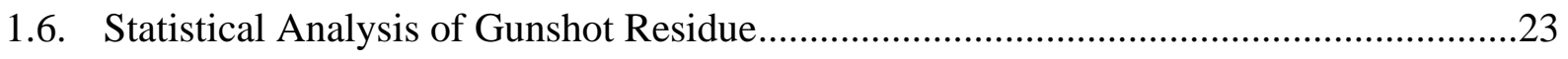

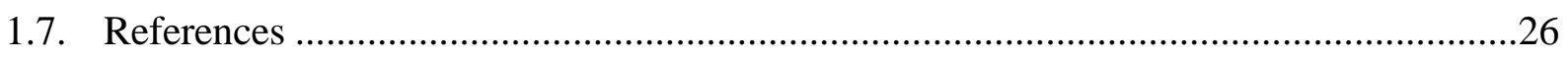

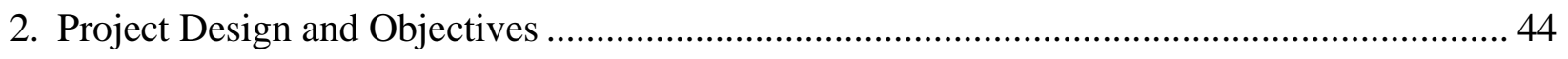

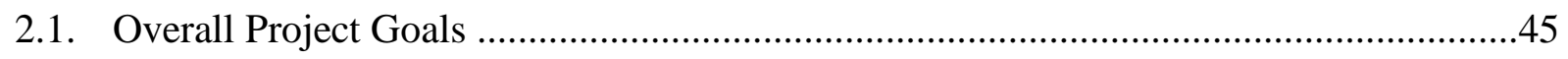

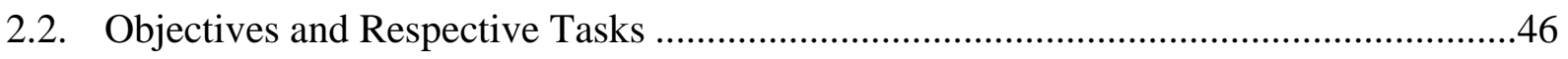

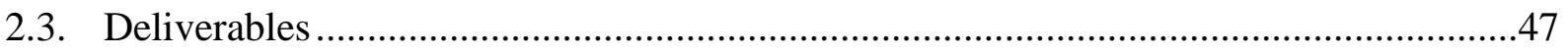

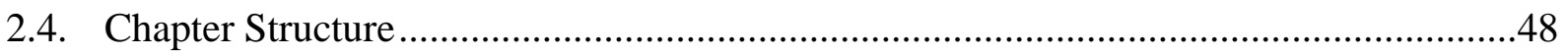

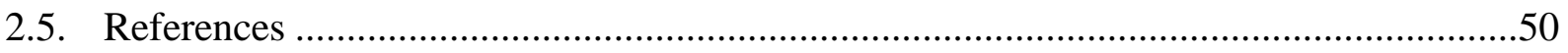

3. Chapter 1: Development and Validation of an ICP-MS Digestion Method for the Bulk Characterization of Tailor-Made IGSR Standards ................................................................. 51

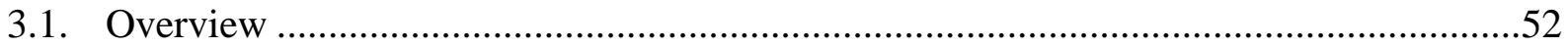

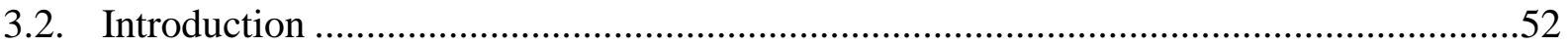

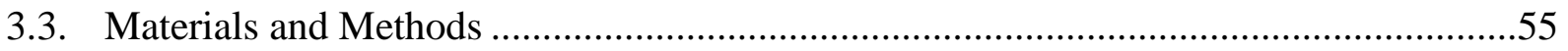




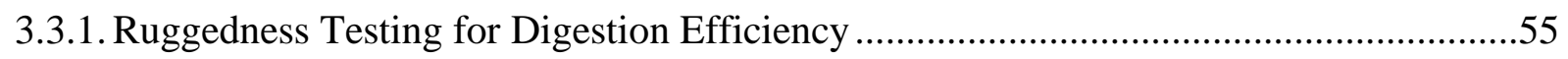

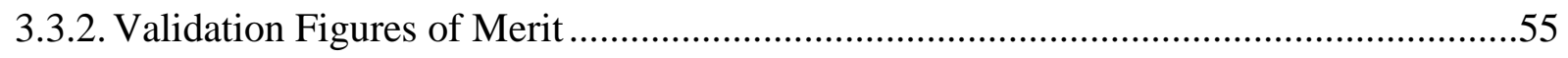

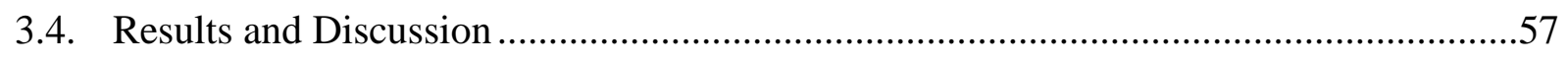

3.4.1. Ruggedness Testing of ICP-MS Digestion Methods...................................................57

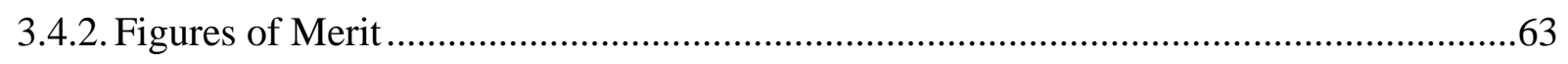

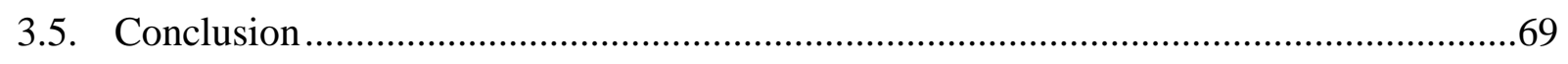

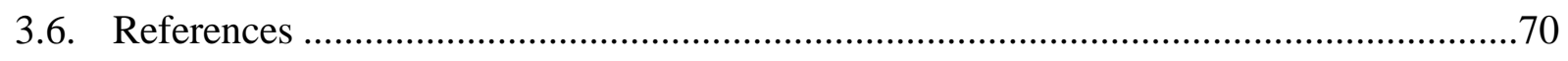

4. Chapter 2. Multi-Technique Approach to Characterization of the Tailor-Made IGSR

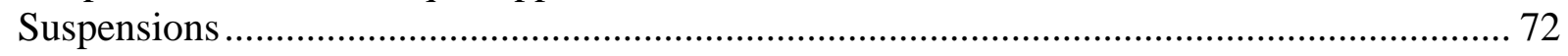

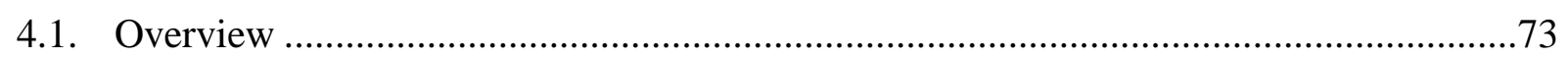

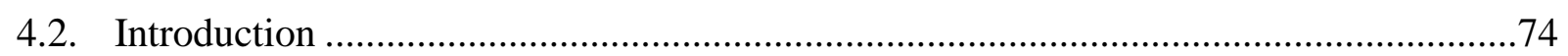

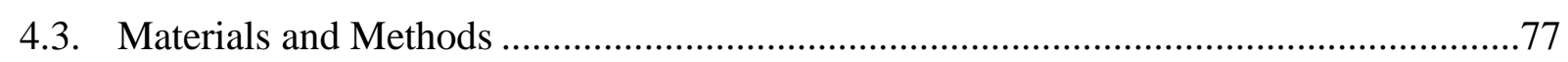

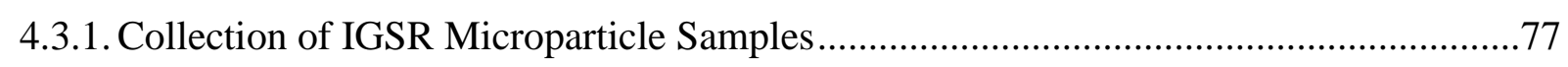

4.3.2. ICP-MS Analysis on IGSR Microparticle Samples .......................................................

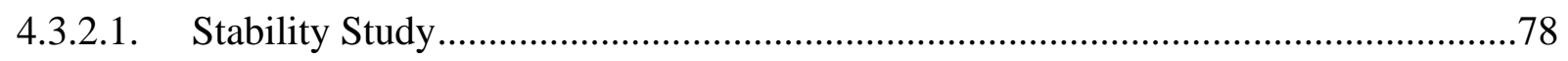

4.3.2.2. ICP-MS Instrumental Parameters …………….................................................

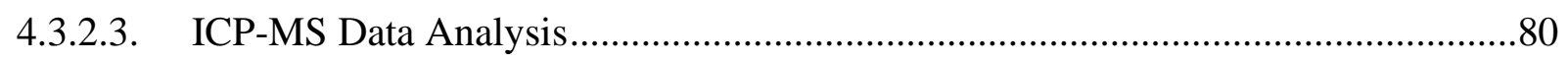

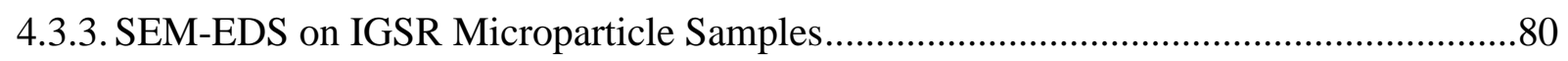

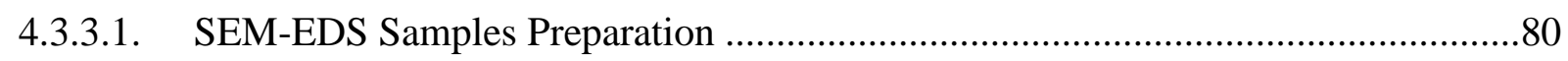

4.3.3.2. SEM-EDS Instrumental Parameters …………………................................... 81

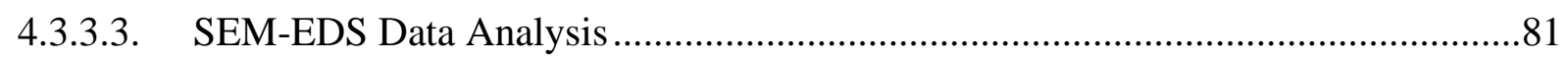

4.3.4. LIBS Application of IGSR Microparticle Samples .................................................... 82

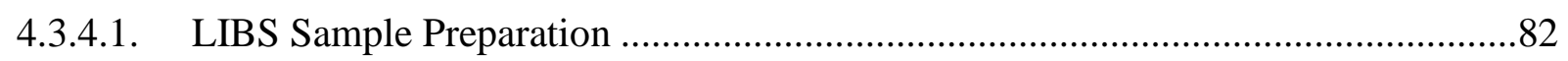

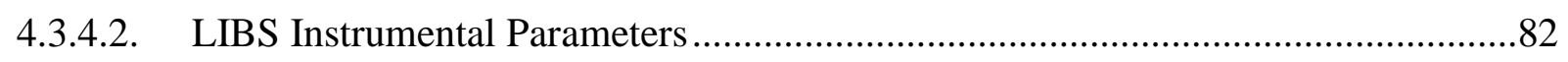

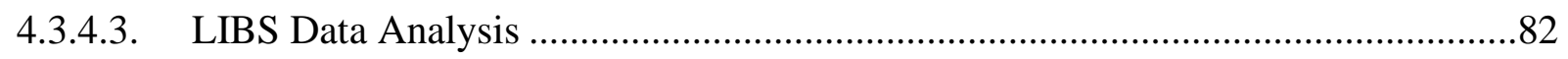

4.3.5. Collection of Known-Shooter Samples for Comparison ................................................. 83

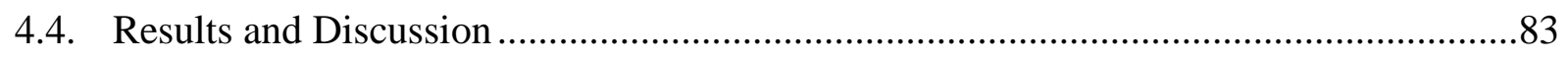

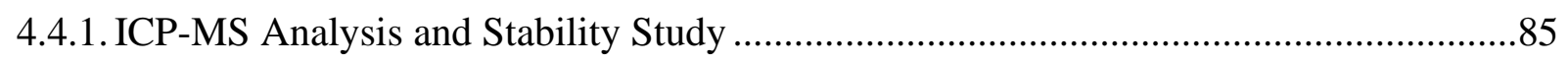

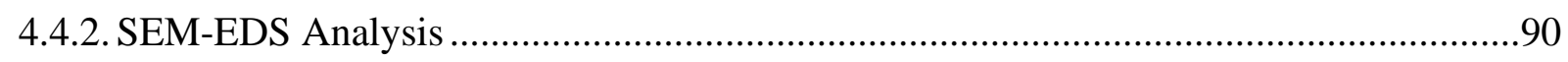

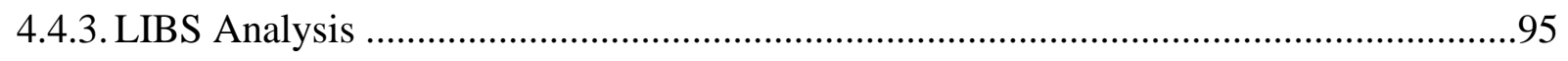

4.4.4. Comparison of the Microparticle Standards to IGSR Collected from Known Shooter's Hands . . . . .

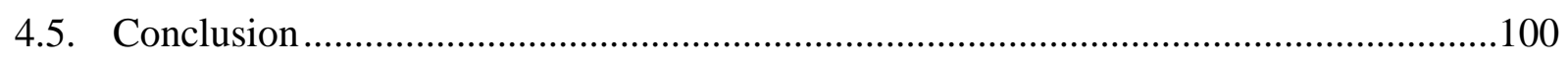

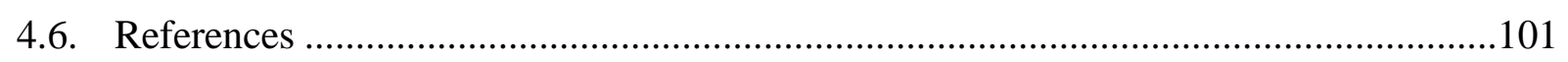

4.7. Expanded Characterization of the Microparticle IGSR Standards ..................................109 
5. Chapter 3: Development of the Rapid LIBS Micro-Spatial Method for the Detection of IGSR

5.1. Overview .

5.2. Introduction .122

5.3. Materials and Methods ..... .125

5.3.1. Sample Preparation for Method Optimization .125

5.3.2. Firearms and Ammunition Used for the Validation Study. 125

5.3.3. Sample Preparation and Collection of Residues from Shooter and Non-Shooter's Hands.

5.3.4. LIBS Experimental Setup. .127

5.3.5. Data Preprocessing and Statistical Analysis 129

5.4. Results and Discussion 131

5.4.1. Spot Micro-Sampling Method Optimization. .131

5.4.2. Performance Comparison of Ablation Methods and Classification Approach 133

5.5. Conclusion. 139

5.6. References 140

6. Chapter 4: Gunshot Residue Population Study of Hands 145

6.1. Overview 146

6.2. Introduction 147

6.2.1. Current versus Bayesian Interpretation for GSR Evidence 150

6.2.2. Goals of the Population Study 152

6.3. Materials and Methods 152

6.3.1. Sample Collection for the Population Study ...........................................................152

6.3.2. Quality Control Samples for Instrument Daily Performance ....................................159

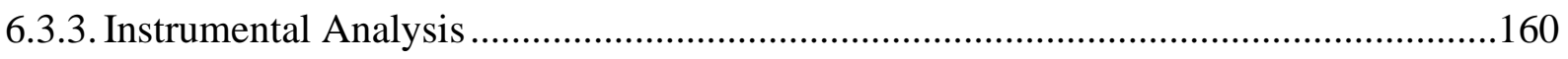

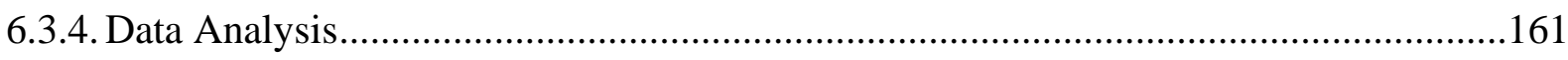

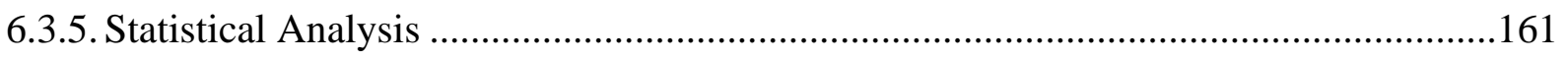

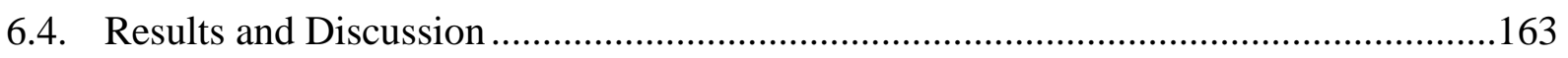

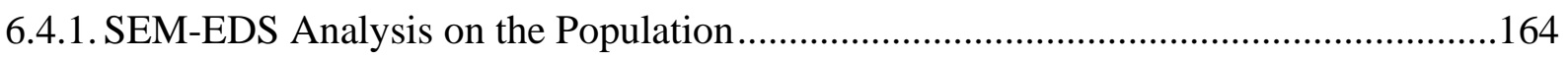

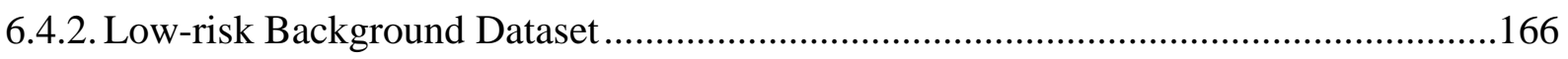

6.4.3. Shooter hands' Residues from Leaded Ammunition Dataset.....................................167

6.4.4. Shooter hands' residues from lead-free ammunition dataset ....................................167

6.4.5. LIBS Analysis on the Population Sets............................................................... 178

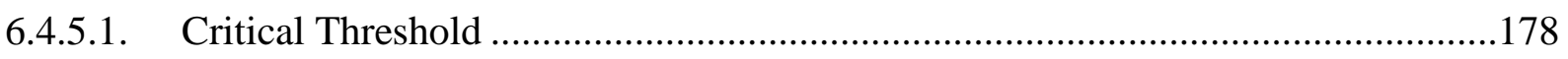


6.4.5.2. Machine Learning Algorithms for Classification of GSR and Non-GSR Residues.... 184

6.4.5.3. Probabilistic Interpretation of the Populations 185

6.5. Conclusion. 192

6.6. References 193

7. Overall Conclusions and Future Directions 200

7.1. Summary of the Study .201

7.1.1. Objective 1: Qualitative and Quantitative Characterization of Multiple Types of Standard and Lead-Free Primers using a Novel Collection and Analytical Approach .201

7.1.2. Objective 2: Validation of the Reliability of LIBS and SEM-EDS by the Statistical Analysis of a Large Population .203

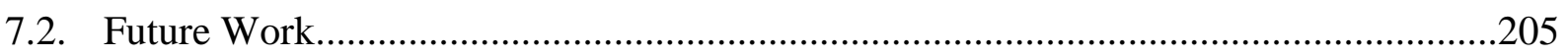

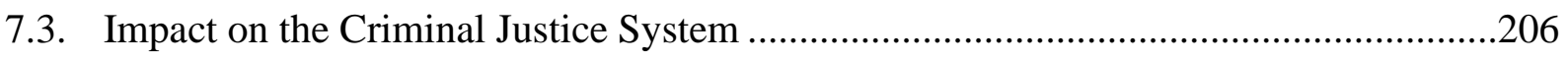

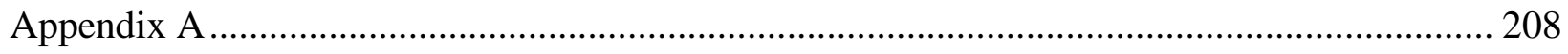




\section{Section 1}

Figure 1: Historical Timeline of GSR analysis.

Figure 2: A) Schematic of a typical ICP-MS with a zoomed in schematic of the sample introduction area (more detailed view from 65) B) Schematic of the construction and working of various components located within the electron column and specimen chamber of the SEM C)

Schematic representation of a typical LIBS setup. 13

Figure 3: Diagram of the interaction volume for the electron beam and different types information emitted. 15

\section{Section 2}

Figure 1: Flowchart of project implementation and method. Objective $=\mathrm{Ob}$. Task=T 45

\section{Section 3: Chapter 1}

Figure 1: Correlation plot of all main effects tested for Plackett-Burman ruggedness testing. The plot visually demonstrated the correlation between each factor at two different levels with a cross-wise comparison of the different factors at the different levels. Each square is a comparison point, and the plot shown here shows low correlation between different factors. The diagonal red square down the center are the point when the same factor is compared with itself, therefore a high correlation is to be expected.

Figure 2: Average standardized effects chart of Plackett-Burman ruggedness testing. 61

Figure 3: Chart displaying the inter- and intraday variability of the method at $25 \mu \mathrm{g} / \mathrm{L}$. Each

analyte was monitored on four different day with 6-9 replicate measurements each day. 67

Figure 4: Chart displaying the inter- and intraday variability of the method at $50 \mu \mathrm{g} / \mathrm{L}$. Each analyte was monitored on four different day with 6-9 replicate measurements each day. 68

\section{Section 4: Chapter 2}

Figure 1: ANOVA and Tukey-Kramer results for two in-house primer-only samples. The results confirm the elements of interest (possible GSR) are remaining stable during the 12-week storage period. 87 
Figure 2: Inceptor primer-only data from 12-week ICP-MS study ANOVA and Tukey-Kramer results. Ti and $\mathrm{Zn}$ are common elements in lead-free ammunition, and $\mathrm{Sb}, \mathrm{Ba}$, and $\mathrm{Pb}$ are common elements in leaded ammunition. The replicates collected show both types of GSR elements.

Figure 3: Examples of microparticles after 12-weeks of stability. Top is a particle generated by Winchester: standard, leaded primer with antimony, barium, and lead. Bottom is a particle generated by TULAMMO: standard, leaded primer that lacks barium. The imbedded images were taken using SEM and spectrum was generated using EDS 93

Figure 4: Examples of microparticles after 12-weeks of stability. Top is a particle generated by Fiocchi: lead-free primer with only a light atomic weight element as a GSR elements. Bottom is a particle generated by SYNTECH: lead-free primer with only one heavy atomic weight element as a GSR element. Both microparticles have common background elements (aluminum in both and silicon-Fiocchi only) incorporated into the particle. The imbedded images were taken using SEM and spectrum was generated using EDS. 94

Figure 5: Example of SEM-EDS particle mapping for a $2 \mu \mathrm{L}$ deposit of a TULAMMO microparticle reference suspension containing a total of 325 particles and SEM images of the varying sizes of particles in the microparticle reference suspension. 95

Figure 6: Spectral comparison of Syntech ammunition and the detection sensitivity of Bi with two different types of detectors and the same J200 LIBS configuration.

Figure 7: GSR particles collected from the hands of known-shooters with ammunition using the same brand and lot of primers as the IGSR microparticle standards created. The IGSR microparticles collected with only a primer and a cartridge case exhibit the sample elemental composition and morphology as known-shooter samples collected from the hands. Winchester is a leaded ammunition and Fiocchi is a lead-free ammunition. 99 Figure 8: ANOVA and Tukey-Kramer for two IGSR in-house primer-only standards. The results confirm the elements of interest (possible GSR markers) are remaining stable during the one-year storage period.

Figure 9: Example of a primer-only particle generated by TULAMMO: standard, leaded ammunition that lacks barium.

Figure 10: Example of particle generated by Fiocchi: nontoxic ammunition that lacks any characteristic GSR markers. 


\section{Section 5: Chapter 3}

Figure 1: Neural Network diagram. The input from the five elements is processed by each node (green circles) within the single hidden layer. The output is the form classification of shooter vs non-shooter. 130

Figure 2: Comparison of the pattern and spectral information for the two ablation methods. The line method only produces one accumulated spectrum for the $7 \mathrm{~mm}$ by $100 \mathrm{um}$ line pattern. The spot method produces one spectrum for each 100 um in diameter spot result in 25 spectra per pattern. The darker blue the square in the heat map, the higher signal-to-noise ratio (SNR) for that element.

\section{Section 6: Chapter 4}

Figure 1: Flowchart of the samples collected for the known-shooter population of the study. Each set consists of four samples: right back, right palm, left back, and left palm. 156

Figure 2: Flowchart of the samples collected for the background population of the study. Each set consists of two stubs: right hand and left hand, except for the 50 samples collected in the Mountainlair from only the dominant hand.

Figure 3: Low-risk background population and the classification with rankings for the total number of particles found on all the stubs analyzed $(\mathrm{n}=56)$.

Figure 4: SEM-EDS spectrum and image of the only characteristic GSR particle identified during the confirmation of low-risk background hand samples.

Figure 5: SEM-EDS spectrum and image of a spherical microparticle observed on a low-risk background samples during confirmation. 170

Figure 6: SEM-EDS spectrum and image of a Fiocchi shooter sample observed during the confirmation of the lead-free known shooter samples.

Figure 7: Leaded known shooter population and the classification with rankings for the total number of particles found on all the stubs analyzed $(n=52)$.

Figure 8: Lead-free known shooter from CCI ammunition and the classification with rankings for the total number of particles found on all the stubs analyzed $(n=19)$. 174 Figure 9: SEM-EDS spectrum and image of irregular (top) and spherical (bottom) CCI GSR particles identified during the confirmation of lead-free known shooter hand samples. 175 
Figure 10: Lead-free known shooter from SYNTECH ammunition and the classification with rankings for the total number of particles found on all the stubs analyzed $(n=14)$.

Figure 11: Lead-free known shooter from Fiocchi ammunition and the classification with rankings for the total number of particles found on all the stubs analyzed $(n=17)$.

Figure 12: Histograms displaying the distribution of likelihood ratio calculated from logistic regression (left) and a neural network (right). The likelihood ratio 1 equation was used for calculation. $\mathrm{H}_{1}$ is the prosecutor's hypothesis and $\mathrm{H}_{2}$ is the defense hypothesis. 188

Figure 13: Tippet plots displaying the proportion of misleading cases from logistic regression (left) and a neural network (right). The likelihood ratio 1 equation was used for calculation. $\mathrm{H}_{1}$ is the prosecutor's hypothesis and $\mathrm{H}_{2}$ is the defense hypothesis. Where the line plotting the likelihood ratio of the $\mathrm{H}_{p} / \mathrm{H}_{1}$ crosses the dotted line at $\log \mathrm{LR} 0$, the integrated area between the line and dotted line at the top is the rate of misleading evidence for that hypothesis, or also called the false negative rate. The opposing hypothesis is where the line plotting the likelihood ratio of the $\mathrm{H}_{d} / \mathrm{H}_{2}$ crosses the dotted line at 0 , the integrated area between the line and dotted line at the bottom is the rate of misleading evidence for that hypothesis, or also called the false positive rate.

Figure 14: Histograms (top) with the corresponding KDF below. From left to right: leaded shooter and low risk populations, mixed shooter and low risk populations, lead free and low risk populations, and all shooter and low risk populations. 


\section{Section 1}

Table 1: Classification Scheme for IGSR (Adapted from ASTM E1588-20) .......................... 5

Table 2: Different types of primer compositions analyzed by SEM-EDS to determine the elemental combinations present in the GSR particles created. 7

Table 3: Comparison of Common Techniques for Characterization of Firearm Discharge

Residues 11

\section{Section 3: Chapter 1}

Table 1: Factors and levels chosen for the Plackett-Burman experimental design. 54

Table 2: Standardized effects calculation table for Plackett-Burman ruggedness test ..... 59

Table 3: Alias matrix of Plackett-Burman ruggedness test.

Table 4: Figures of merit for the validation study of the acid digestion- ICP-MS method for analysis of gunshot residues from different primer in-house standards. 66

\section{Section 4: Chapter 2}

Table 1: List of the different primer types collected for the study. 78

Table 2: Summary of the elemental concentration for the ten primers collected (replicate B; $n=4$ each) 86

Table 3: Summary of the elements detected in the IGSR microparticle suspensions separated by all techniques and primer type.

Table 4: Summary of the elements and morphology detected in the IGSR microparticle suspensions separated by all techniques and primer type. 100

Table 5: List of the different primer types collected for characterization of the tailor-made IGSR standards. Each primer type was collected in triplicate.

Table 6: Summary of the elemental concentration for the ten leaded and five lead-free IGSRprimer standards collected and values represent mean concentrations observed over 52-weeks. Those with an asterisk (*) have only been monitored for 24-weeks and are still being monitored.

Table 7: Summary of the elements observed by SEM-EDS analysis. 118 
Table 8: Summary of the elements detected in the IGSR microparticle solutions by all techniques for different primers.

\section{Section 5: Chapter 3}

Table 1: Summary of ammunition and firearms used in the study ....................................... 126

Table 2: Box-Behnken experimental design for micro-spot method optimization..................... 128

Table 3: LIBS optimized parameters used for the ablation methods validation study ............... 129

Table 4: Comparison of the statistical analysis and performance measures for validation study.

\section{Section 6: Chapter 4}

Table 1: Example of hierarchy of proposition for GSR evidence. Adapted from Maitre et al.. 152

Table 2: Summary of the different types of firearm and ammunition used for the standard

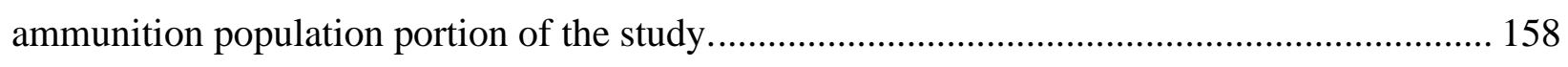

Table 3: List of the monitored emission lines per element.................................................... 179

Table 4: Critical threshold results for the leaded shooter $(n=150)$ and low-risk background $(\mathrm{n}=110)$ for a subset of the shooter-leaded population by LIBS.

Table 5: Summary of the positive calls criteria for the known shooter samples collected. A set was considered positive if the GSR marker combination specified was identified within a single ablation spot.

Table 6: Summary table of the difference between the number of positive sets depending on the positive criteria set.

Table 7: List of all the positive samples from the high-risk population and the elements observed (false positive). While $\mathrm{Cu}$ is not typically used to identify GSR, it is a major element in lead-free FIO and was monitored in this study. These samples are awaiting confirmation by SEM-EDS. Legend; FRA is firearm/impression research laboratory, AGL is farm and greenhouse, MEC is mechanic, and POL is police/station. 183

Table 8: Element and monitored wavelength (nm) used in the training of the machine learning algorithms and separated by the type of the ammunition the elements assist in classifying. * Mixed: leaded and lead-free group elements in addition to the elements listed here...... 185 
Table 9: Comparison of performance rates with the increase in the number of variables (elements and wavelengths) monitored to assist in the classification of modern, lead-free ammunition (350 low-risk backgrounds, 200 leaded shooters, 100 lead-free shooters, and 220 mixed shooters). 185

\section{Table of Equations}

\section{Section 3: Chapter 1}

Equation 1: Formula for LOD calculation with the standard deviation $\left(s^{\prime} 0\right)$ from the experimental standard deviation $(s 0)$, the number of replicate observations subjected to the entire procedure $(n)$, and the number of observations used to calculate the blank correction $(n b)$ during analysis.

Equation 2: Formula for relative percent bias using the average of the replicate samples subjected to the method $(x)$ and the target value spiked into the samples ( $x$ ref). 57

Equation 3: Formula for relative percent recovery using the average of the replicate samples subjected to the method $(x)$ and the target value spiked into the samples ( $x$ ref).

\section{Section 5: Chapter 3}

Equation 1: Formula for the Naïve Bayes Formula. 135

\section{Section 6: Chapter 4}

Equation 1: Likelihood ratio formula 1 where the shooter changes based on the population being considered (leaded, lead-free, or mixed)...... 163

Equation 2: Likelihood ratio formula 2 where all three shooter populations are considered simultaneously. 


\section{Section 1}

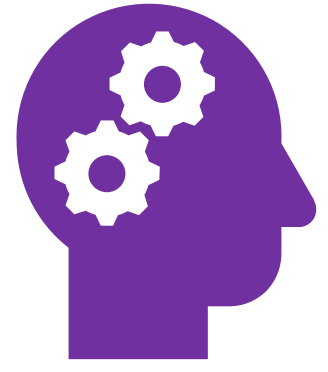

Problem statement

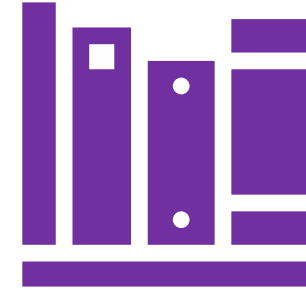

Literature Review

Research

focus

Problem Statement, Introduction, and Literature Review 


\section{Introduction}

\subsection{Problem Statement}

The most recent data from the United States (US) National Crime Victimization Survey (NCVS) reported over 481,000 victims of firearm-related crimes in the year 2019, and out of those, over 65,500 victims were injured. ${ }^{1}$ During the investigation of these crimes, residues collected from individuals of interest are commonly submitted to forensic laboratories to identify Gunshot Residue (GSR). ${ }^{2,3}$ Based on the presence or absence of GSR, a forensic examiner can make inferences about whether or not the individual could have been in the vicinity of a shooting event. These decisions are made based on the identification of particle morphology and elemental composition by Scanning Electron Microscopy-Energy Dispersive X-ray Spectrometry (SEM$\mathrm{EDS})$. Lead, barium, and antimony ( $\mathrm{Pb}, \mathrm{Ba}$, and $\mathrm{Sb}$ ) have been the primary elements of inorganic GSR (IGSR) as they are commonly found in standard ammunition. ${ }^{4}$ In the last couple of decades, however, lead-free and nontoxic ammunitions have been adopted due to environmental protection regulations and health concerns. ${ }^{5-10}$ Emerging modern ammunitions require an expansion of elemental profiles and thresholds indicative of IGSR.

Since firearm-related crimes pose a particular risk to individual safety, and society, it is essential that violent criminals are detained and receiving swift convictions, while innocents are not being wrongfully held in jails. ${ }^{11-14}$ Expedited and reliable methods not only influence prompt justice, but also reduce incarceration costs by decreasing the time between arrest and resolution. ${ }^{15-}$ ${ }^{18}$ Moreover, fast GSR collection and analysis are ideal due to the short-term persistence of GSR and the susceptibility to exogenous contamination, though these techniques are not readily available to crime laboratories. ${ }^{19-21}$

Consequently, current protocols for examination of GSR evidence face challenges regarding response times and continuous changes to GSR's chemical composition. Foremost, there is still a gap of guidance on the determination of thresholds and statistical information necessary to form definitive conclusions about the evidential value of detecting GSR on an individual. As a result, there is a critical need to overcome all these limitations. In the absence of extended research in these topics, the evidential value of GSR will be likely disputed in court. 
The overall goal of this study is to enhance the reliability of GSR evidence by speeding up the time between collection and detection, expand the body of knowledge on chemical composition of modern cartridge residues, and validating statistical methods for the quantitative assessment of the evidential value of GSR. These goals were achieved through two major objectives: 1) composition characterization of IGSR from modern ammunition, 2) validation of the reliability of Laser Induced Breakdown Spectroscopy (LIBS) and SEM-EDS by the statistical analysis of a population that includes hand residues from characterized ammunitions, and non-shooter background specimens, that contains both low and high-risk background populations.

Studies conducted in our research group have demonstrated that LIBS can provide a costeffective method, and ultra-fast analysis ( $<2$ minutes) for IGSR detection. The method is minimally destructive of the sample, allowing further confirmation by SEM-EDS when needed. Although LIBS lacks high magnification imaging, it can provide spatial chemical mapping of

multi-elemental compositions. ${ }^{22-29}$ It is hypothesized that the incorporation of LIBS will improve current response times, and can be particularly useful in cases where quick decision making is necessary to protect public safety.

The first step of this study is a full characterization of contemporary primers available on in the current market, in order to approach the project with a ground-truth knowledge of expected elemental profiles. This was accomplished with tailored-made GSR microparticle standards created from the discharge of primer-only ammunition and characterized by a multiple-technique approach, which was the ground truth for the authentic samples collected for this study.

This information served as an essential basis of the anticipated elemental GSR composition for a control set of known-shooters, and at the same time allowed the comparison and validation of novel methods (LIBS) and current practice (SEM-EDS). An important aspect of this research was to provide examiners with fundamental information and a statistical approach to strengthen their evidence assessment. In turn, this will hopefully improve the criminal justice system in the U.S. by providing quantitative and objective conclusions when examiners are presenting forensic evidence in court.

\subsection{Composition, Morphology and Detection of Gunshot Residue}

The composition of GSR can be separated into two main categories, the inorganic gunshot residues (IGSR) and the organic gunshot residues (OGSR). The IGSR compounds, such as 
antimony $(\mathrm{Sb})$, lead $(\mathrm{Pb})$, and barium $(\mathrm{Ba})$ are generated by the primer, with some elements, such as aluminum $(\mathrm{Al})$, copper $(\mathrm{Cu})$, and additional $\mathrm{Pb}$, contributed by the bullet and cartridge case. ${ }^{8,20,30,31}$ The majority of OGSR originates from the additives or explosives found in the propellant. Additives - such as flash inhibitors, stabilizers, or plasticizers - are present to extend the shelf-life and performance of the propellant. ${ }^{32-34}$ The smokeless powder in the propellant is characterized by the chemical compound(s) used; nitrocellulose (NC) if single-based, nitrocellulose and nitroglycerin (NG) if double based, and the further addition of nitroguanidine if triple-based powder. ${ }^{35}$ Stabilizers such as diphenylamine (DPA) and ethyl centralite (EC), or a flash suppressor such as 2-4-dinitrotoluenes (2,4-DNT) are also common compounds of interest for the detection of GSR. ${ }^{32-38}$ Nontoxic ammunition will contain very similar OGSR, but distinctive IGSR since the primer is the main source of the no environmentally-friendly heavy metals. ${ }^{31}$

When a firearm is discharged, the primer creates a spark which ignites the propellant and causes a plume of fine particles and material containing IGSR and OGSR. ${ }^{30,39-41}$ The plume of discharge is created by vaporized and partially molten primer and propellant materials at high temperatures $\left(1500-2000^{\circ} \mathrm{C}\right)$ and pressures $\left(\sim 10^{4} \mathrm{kPa}\right)$ within ten thousandth of a second. ${ }^{29,40}$ The rapid heating and cooling of the vaporized materials form spheroid particles due to cohesive properties of the compounds and the extreme temperature and pressures created during the firing process. ${ }^{42,43}$ Studies have also confirmed some irregular particles can be generated, depending on the variations in conditions of formation and the mechanical stress the particles may undergo. ${ }^{31,39,41}$ Current practice relies on identification of IGSR particles exhibiting distinctive elemental composition and spheroid shape, typically ranging in size from $0.5-5 \mu \mathrm{m} .{ }^{4}$

Similarly, the elemental composition of GSR can vary depending on the specific properties of the cartridge, including the manufacturer, design, and end use of the ammunition (e.g., target practice versus self-defense). A majority of standard ammunition utilizes lead styphnate as the initiator, barium nitrate as the oxidant, and antimony sulfide as the fuel, producing IGSR particles that contain $\mathrm{Pb}, \mathrm{Ba}$, and $\mathrm{Sb}$ elements. ${ }^{22,25,28,41,44}$ Non-toxic ammunition manufacturers can replace the standard heavy-metal compounds with strontium ( $\mathrm{Sr}$ ) or potassium $(\mathrm{K})$ oxidizers and other claimed environmentally friendly ingredients, such as diazodinitrophenol or calcium silicide. ${ }^{5,9,45,46}$ In the current ASTM standard for GSR evidence analysis, gadolinium (Ga), titanium (Ti), and zinc ( $\mathrm{Zn}$ ) are listed as some of the inorganic elements to identify non-toxic 
IGSR. ${ }^{4}$ According to the current standard, the listed elements must be found in certain combinations within a single particle in order for it to be considered GSR (Table 1). ${ }^{4}$ Even more challenging, a sample collected from someone who was not involved in a crime could still possess the elemental composition associated with IGSR, and this will be discussed in further detail throughout this document and specifically in section 1.5.

Confirmation of GSR is not a straightforward task, and the ASTM standard provides some guidelines for the analysis and interpretation of evidence. Since GSR is created under atypical conditions (e.g., rapidly, at high temperature and pressure), the elemental components must be present in the specific combinations described in Table 1 and within a particle generated during the firearm discharge. According to ASTM, identification of the GSR is related to the combination of elements confirmed by the EDS spectrum and an image of the particle used to collect the spectrum. The strongest confidence in a conclusion that a residue is GSR as opposed to other nonGSR trace, is the presence of a 'characteristic' GSR particle, which contains three or more elements of interest depending on the type of ammunition. A weaker identification would be a 'consistent with GSR' particle, which contains two out of three main elements, or one of them in

Table 1: Classification Scheme for IGSR (Adapted from ASTM E1588-20)

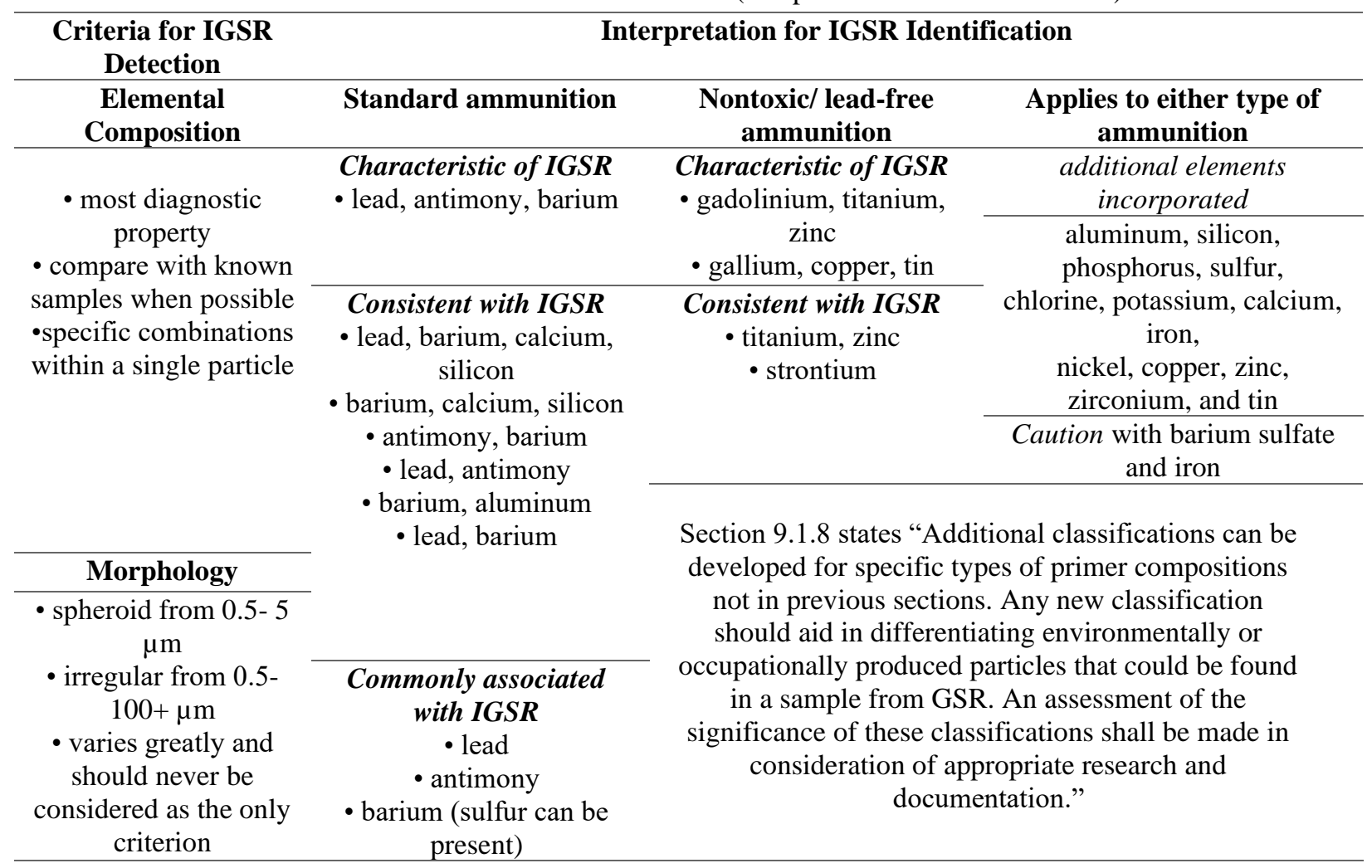


combination with secondary elements, such as calcium $(\mathrm{Ca})$ or silicon $(\mathrm{Si})$. Finally, the weakest identification would be a particle 'commonly associated with GSR' which is finding only one of the main elements $(\mathrm{Pb}, \mathrm{Ba}$, or $\mathrm{Sb})$. The current standard does not provide a criteria on 'commonly associated with' elemental combinations for non-toxic or lead-free ammunitions. ${ }^{4}$

Nontoxic ammunition has been adopted in European countries for over a decade, due to environmental and health concerns about the heavy metal contamination. 5,6,8,32,34 Consequently, more of these modern primers are also being seen in crime laboratories as the US adopts similar practices. For example, California began enforcement on July 1, 2019 of a ban on leaded ammunition for hunting and sport. ${ }^{47}$ For California, and other states enforcing these policies, the use of more nontoxic ammunition will create a new challenge for evidence interpretation. Therefore, it is necessary to update the current knowledge about industry available ammunition to increase the strength of the standards being applied to IGSR analysis. Nontoxic and heavy metalfree ammunition are a growing market, but the composition and behavior of the GSR of these modern ammunition has only begun to be researched. ${ }^{6-9,28,48-50}$ A summary of some nontoxic ammunitions studied in the past and with known primer compositions can be found in Table 2 .

Determining the original primer composition is challenging due to the proprietary nature of ammunition manufacturing. Still, without a thorough understanding of the elemental composition of nontoxic ammunition, and alternate method of detection, the field of GSR analysis is vulnerable to a large uncertainty in the interpretation of modern GSR.

Morphology, along with elemental composition, is also important for GSR identification. The shape and size of GSR particles assists in the differentiation of non-GSR sources that may share similar elemental compositions, and can be present in the background population as particles that mimic GSR morphology (more detail in section 1.5). ${ }^{453-55}$ GSR particles are commonly a particle on the micrometer scale and possess a spheroid shape as a result of the firearm discharge condition. ${ }^{31,42}$ However, studies have published images of nontoxic GSR particles that do not possess the spherical shape of standard GSR particles, and the current reasoning is the effect of the new elements in the ammunition. 7,39,44,50

The distribution of the elements and compounds within a GSR particle are also a relevant area of research. When analyzing GSR, some particles may only contain two of the three elements present int the primer, even when the particles are known to have originated from discharging a 
Table 2: Different types of primer compositions analyzed by SEM-EDS to determine the elemental combinations present in the GSR particles created.

\begin{tabular}{|c|c|c|c|c|}
\hline Ammunition & Elements & Primer Composition & $\begin{array}{l}\text { Residue } \\
\text { Particles }\end{array}$ & Reference \\
\hline Sintox & $\mathrm{Ti}, \mathrm{Zn}, \mathrm{Pb}, \mathrm{Ba}$ & $\begin{array}{l}\text { Diazodinitrophenol (DDNP), tetracene, zinc } \\
\text { peroxide, titanium } \\
\text { metal powder, nitrocellulose }\end{array}$ & $\begin{array}{l}\mathrm{Zn}-\mathrm{Ti} \\
\mathrm{Zn}-\mathrm{Pb} \\
\mathrm{Ti}-\mathrm{Zn}-\mathrm{Pb}-\mathrm{Ba}\end{array}$ & 47 \\
\hline $\begin{array}{l}\text { CCI Blazer }{ }^{\circledR} \\
\text { Lead free }\end{array}$ & $\mathrm{Sr}, \mathrm{Ba}$ & $\begin{array}{l}\text { Tetracene, DDNP, smokeless powder, strontium } \\
\text { nitrate }\end{array}$ & $\mathrm{Sr}, \mathrm{Sr}-\mathrm{Ba}$ & 48 \\
\hline $\begin{array}{l}\text { Winchester } \\
\text { WinClean }\end{array}$ & $\begin{array}{l}\mathrm{K}, \text { trace } \mathrm{Al}, \mathrm{Si} \\
\mathrm{Ca}, \mathrm{S}, \mathrm{Zn}, \mathrm{Cu} \\
\mathrm{Ni}, \mathrm{Cr}, \mathrm{Mg}, \mathrm{Fe}\end{array}$ & $\begin{array}{l}\text { Copper and zinc (primer cup), DDNP, potassium } \\
\text { nitrate, boron, nitrocellulose (MSDS contents) }\end{array}$ & $\begin{array}{l}\mathrm{Cu}-\mathrm{Zn} \\
\mathrm{K}-\mathrm{Al}-\mathrm{Si}-\mathrm{Na} \\
\mathrm{Al}-\mathrm{Na} \\
\mathrm{Ca} \text { and } \mathrm{Mg}\end{array}$ & 49 \\
\hline $\begin{array}{l}\text { Remington/ } \\
\text { UMC Leadless }\end{array}$ & $\begin{array}{l}\mathrm{Al}, \mathrm{Si}, \mathrm{K}, \mathrm{Na} \\
\mathrm{Ca}, \text { trace } \mathrm{Mg}\end{array}$ & $\begin{array}{l}\text { Copper and zinc (primer cup), DDNP, barium, } \\
\text { tetracene (MSDS contents) }\end{array}$ & $\begin{array}{l}\mathrm{Cu}-\mathrm{Zn} \\
\mathrm{Al}-\mathrm{Si}-\mathrm{K}(\mathrm{Na} \text { or } \\
\mathrm{Ca}) \\
\mathrm{Al}-\mathrm{Si}-\mathrm{K} \text { (trace } \\
\mathrm{Na})\end{array}$ & 49 \\
\hline $\begin{array}{l}\text { Speer } \\
\text { Lawman } \\
\text { CleanFire }\end{array}$ & $\mathrm{Sr}, \mathrm{O}, \mathrm{Al}, \mathrm{Cl}$ & $\begin{array}{l}\text { Copper, Zinc, nickel, DDNP, tetracene, strontium } \\
\text { nitrate, nitrocellulose, nitroglycerine }\end{array}$ & $\begin{array}{l}\mathrm{Cu} \\
\mathrm{Cu}-\mathrm{Zn} \\
\mathrm{Sr}-\mathrm{Al} / \mathrm{Si} \text { or } \mathrm{Cl}\end{array}$ & 49 \\
\hline $\begin{array}{l}\text { CleanRange } \\
\text { 1st generation }\end{array}$ & $\mathrm{Sr}, \mathrm{Na}, \mathrm{K}, \mathrm{Fe}$ & $\begin{array}{l}\text { DDNP, tetracene, nitrocellulose, strontium } \\
\text { nitrate, ground glass, calcium carbonate, gum } \\
\text { tragacanth (patent claim) }\end{array}$ & $\begin{array}{l}\mathrm{Sr} \text { (plume) } \\
\mathrm{Sr}-\mathrm{Na}-\mathrm{K}-\mathrm{Fe} \\
\text { (hand residue) }\end{array}$ & 50 \\
\hline $\begin{array}{l}\text { CleanRange } \\
\text { 2nd generation }\end{array}$ & $\mathrm{Al}, \mathrm{Si}, \mathrm{K}, \mathrm{Ca}, \mathrm{S}$ & $\begin{array}{l}\text { DDNP, tetracene, nitrocellulose, potassium } \\
\text { nitrate, aluminum powder, ground glass, calcium } \\
\text { carbonate, gum tragacanth (patent claim) }\end{array}$ & $\begin{array}{l}\mathrm{Al}, \mathrm{Si}, \mathrm{Ca} \text { (plume) } \\
\mathrm{Al}-\mathrm{K}-\mathrm{Si}-\mathrm{Ca}-\mathrm{Fe} \\
(\mathrm{S} \text { in } 0.38 \mathrm{SPL}) \\
\text { (hand residue) }\end{array}$ & 50 \\
\hline $\begin{array}{l}\text { Federal } \\
\text { BallistiClean }\end{array}$ & $\mathrm{Ba}, \mathrm{Si}, \mathrm{Al}$ & $\begin{array}{l}\text { Copper and zinc (primer cup), tetracene, barium } \\
\text { nitrate, aluminum, nitrocellulose, nitroglycerine }\end{array}$ & $\begin{array}{l}\mathrm{Al}, \mathrm{Si}, \mathrm{Ca} \text { (plume) } \\
\mathrm{Al}-\mathrm{K}-\mathrm{Si}-\mathrm{Ca}-\mathrm{Fe} \\
\text { ( } \mathrm{S} \text { in } 0.38 \mathrm{SPL} \text { ) } \\
\text { (hand residue) }\end{array}$ & 49 \\
\hline
\end{tabular}

firearm. Heterogeneous distribution of the elements within a sample spurred further research by a group on the surface chemistry of GSR. ${ }^{31,43}$ The study by Schwoeble et. al. found a large variation in not only the distribution of $\mathrm{Pb}, \mathrm{Ba}$, and $\mathrm{Sb}$ between particles, but the elemental combinations and variations which exist in a GSR particle. The study observed changes of the species within a particle as the depth of analysis was changed. For example, $\mathrm{Pb}$ on the surface of a particle tends to exist as only as ionic $\mathrm{Pb}$, but the deeper the analysis, more of the $\mathrm{PbO}$ species are detected. ${ }^{31}$

Multiple studies by Kara and research group, examined the morphological and chemical differences between different types of ammunition and the relationship between the size of the particle and the relation to the Boltzmann distribution. ${ }^{39,43}$ In one study, eight different types of pistol ammunition were tested and the elemental composition along with morphology was recorded. The study found that less than half of the particles created during a firing event and counted by the automated software were actually spheroid in shape, the rest of the particles could be irregular, hollow, spongy, half-spheroid, two spheroids with a bridge, or elliptical. Also, the 
elemental composition of the eight different types of ammunition did all contain $\mathrm{Pb}, \mathrm{Ba}, \mathrm{Sb}, \mathrm{Al}$, and $\mathrm{Zn}$, and all but one type of ammunition contained $\mathrm{Cu}$. Then there were additional elements that varied by the ammunition type such as $\mathrm{S}, \mathrm{Ca}, \mathrm{K}, \mathrm{Mg}, \mathrm{Si}, \mathrm{Cl}, \mathrm{Zr}$, Mo, and P. ${ }^{39}$ Another study determined that a large majority of GSR particles range from $0-5 \mu \mathrm{m}$, with a steep decline in the number of particles greater than $2 \mu \mathrm{m} .{ }^{43}$ The variability and heterogeneous distribution of elements could be greatly affected by the chemical and physical properties of the compounds used in any ammunition, therefore changing the distribution of the GSR particles recovered after a firing event.

When a firearm is discharged, there is also an abundance of OGSR present from the propellant, and a few from the primer. ${ }^{32,34,37,46}$ Numerous studies have shown the presence of OGSR of interest — such as 2,4-DNT, DPA, EC, MC, and NG—on the hands of control-shooters using Raman micro-spectroscopy ${ }^{56,57}$, Liquid Chromatography-Mass Spectrometry (LC-MS) ${ }^{21,58-}$ 61, and electrochemistry ${ }^{36,62}$. A preliminary study conducted in our research lab examined the combination of LIBS and electrochemistry for the analysis of GSR. The study resulted in a combined accuracy of $98 \%$, while the techniques individually showed accuracy of $83 \%$ and $75 \%$ respectively. ${ }^{23}$ The additional information provided by OGSR analysis increases the discriminating power of the analytical scheme, while still consuming minimal sample to conduct further analysis by SEM-EDS, if needed.

\subsection{Analytical Methods for Detection and Identification of GSR}

\subsubsection{Overview of Methods}

The three main categories of analytical tests used for the detection and identification of gunshot residues are a) methods of detection of inorganic components, b) methods of detection of organic compounds, and c) methods capable of simultaneous or sequential detection of IGSR and OGSR. Inorganic detection of gunshot residues has been reported by color tests, ${ }^{63}$ neutron activation analysis (NAA), ${ }^{64}$ atomic absorption spectroscopy (AAS), ${ }^{65-67}$ ICP-based methods, ${ }^{7,68-}$ ${ }^{75}$ SEM-EDS, ${ }^{39,40,43,50}$ and LIBS. ${ }^{22,23,25,28,76-78}$ The study of organic gunshot residues has also been accomplished through color assays, ${ }^{79,80}$ infrared spectroscopy techniques, ${ }^{48,56,81-83}$ and mass spectrometry (MS) coupled to gas chromatography (GC), ${ }^{84-87}$ liquid chromatography (LC), ${ }^{32,58,88-}$ 91 and Ion Mobility Spectrometry (IMS). ${ }^{35,61,86,92}$ Fewer techniques have shown the flexibility for 
dual detection of IGSR and OGSR. Most recently, electrochemistry, ${ }^{23,36,62}$ Electrospray-Trapped Ion Mobility-Mass Spectrometry (ESI-TIMS-MS) ${ }^{61}$ specialized LC/MS methods, ${ }^{59,60}$ Raman, ${ }^{46,48,56,57,93}$ and Mass Spectrometry Imaging (MSI) ${ }^{90,94}$ have been successfully applied to the simultaneous detection of inorganic and organic compounds. A brief description of the methods' capabilities and limitations will be discussed in this section, with a greater emphasis on the current state of techniques proposed in this study (SEM-EDS and LIBS). Table 3 lists a comparison of capabilities and limitation of analytical methods for GSR detection. Detection of GSR has evolved over time, and a visual of this progress can be seen in Figure 1.

Color tests have long been used as a preliminary screening of substrates suspected to contain GSR, and often used during the course of investigation due to rapidness of testing and ease of use. ${ }^{40}$ The Griess color test is still used in determining shooting distance, but not recommended for use on hand samples because of its lack of sensitivity and destructive nature. ${ }^{26,42,45,79} \mathrm{~A}$ positive result will turn an orange color in the presence of nitrites, which is a common by-product of OGSR. ${ }^{29,45,82,95}$ Another commonly used color test is sodium rhodizonate, which produces a pink color in the presence of $\mathrm{Ba}$, and purple color in the presence of lead. ${ }^{24,26,29,82}$ These color tests however are not effective for detection of GSR on individual's hands due to the lack of specificity for GSR and risk of preventing further confirmatory testing since the tests are destructive to the sample. Consequently, most departments and agencies have stopped their use for hands analysis due to high false positive rates. ${ }^{45}$ Presence of these compounds and elements are common in the environment, so it is entirely possible for a suspect or surface to test positive, yet never handled a firearm.

Some of the first reliable and specific techniques used for GSR analysis were AAS and NAA, most commonly used for analysis of IGSR. The instrumentation can quantitatively detect the elements of $\mathrm{Pb}, \mathrm{Ba}$, and $\mathrm{Sb}$ in standard ammunition, despite the lack of morphological information from the techniques. ${ }^{40,65}$ NAA can detect many of the GSR elements of interest, but the technique is challenging to apply in practice due to the neutron source being a nuclear reactor and must be operated by highly trained personal under controlled conditions since it is radioactive. ${ }^{45,82}$ AAS was also applied to the detection of GSR, and was successful at detecting $\mathrm{Pb}, \mathrm{Ba}$, and $\mathrm{Sb}$, but difficulty with extraction techniques for $\mathrm{Sb}$ made quantitation challenging. ${ }^{45}$ Still, the detection capability superseded the color tests being applied since 1959, causing the techniques to be advantageous to their time. ${ }^{82}$ As technology advanced, these techniques were 
replaced by robust and practical ones with the ability to detect more elements simultaneously, with less sample preparation, nondestructive to the evidence, and faster analysis time.

With the advances of Inductively Coupled Plasma (ICP) technology, the forensic laboratories adopted ICP-OES and ICP-MS methods to detect gunshot residues. Benefits of ICPbased methods included multielement quantitative analysis with excellent selectivity and precision and detection at levels that fit the requirements for identifying GSR on authentic samples. Collection of samples typically involved using cotton swabs with an acid solution, which then went through acid digestion before ICP-analysis. This approach represented a limitation of ICP analysis because the elemental concentrations represented a snapshot of the "bulk" composition on the individual's hands, which do not necessarily was distinctive of GSR as opposed to other environmental contaminants.

The advent of SEM-EDS overcome this limitation by conducting elemental analysis on a single microscopic particle. Moreover, the technology allows for automated mapping to discover individual particles within a stub. After a comprehensive evaluation of the efficiency of collection methods compatible with SEM-EDS and assessing the technique's reliability for GSR detection, the method was widely implemented in crime laboratories. A consensus-based standard guide was first developed in 1995 and has gone through periodic revisions, being the most recent the 2020 standard practice. 
Table 3: Comparison of Common Techniques for Characterization of Firearm Discharge Residues

\begin{tabular}{|c|c|c|c|c|c|c|c|c|c|c|c|c|c|}
\hline Method & $\begin{array}{c}\text { Detection } \\
\text { (OGSR or } \\
\text { IGSR) } \\
\end{array}$ & $\begin{array}{c}\text { Limit of } \\
\text { Detection }^{57}\end{array}$ & $\begin{array}{c}\text { Destructive } \\
\text { to the } \\
\text { sample } \\
\end{array}$ & $\begin{array}{c}\text { Sample } \\
\text { Preparation }\end{array}$ & Portable & $\begin{array}{c}\text { Adopted in } \\
\text { crime } \\
\text { laboratories }\end{array}$ & $\begin{array}{c}\text { Relative } \\
\text { cost* }\end{array}$ & $\begin{array}{c}\text { Relative } \\
\text { Analysis } \\
\text { Time } \\
\end{array}$ & $\begin{array}{l}\text { Analysis } \\
\text { Type }\end{array}$ & $\begin{array}{l}\text { Analytical } \\
\text { Selectivity }\end{array}$ & $\begin{array}{c}\text { Morphological } \\
\text { Data }\end{array}$ & $\begin{array}{c}\text { Spatial } \\
\text { information }\end{array}$ & References \\
\hline Colorimetric & Sequential & $>500 \mathrm{ppm}$ & Yes & $\begin{array}{l}\text { Yes, reagent } \\
\text { preparation }\end{array}$ & Yes & Yes & $\$$ & minutes & bulk & poor & No & No & 7,26 \\
\hline NAA & IGSR & sub-ppb & Yes & $\begin{array}{c}\text { Yes, } \\
\text { irradiation }\end{array}$ & No & Rarely & $\$ \$ \$$ & hours & bulk & good & No & No & 45,82 \\
\hline AAS & IGSR & sub-ppb & Yes & $\begin{array}{c}\text { Yes, } \\
\text { digestion }\end{array}$ & No & Rarely & $\$ \$ \$$ & hours & bulk & good & No & No & 45,82 \\
\hline IMS & OGSR & ppb-ppm & Yes & None & No & Some extent & $\$ \$$ & seconds & bulk & fair & No & No & 61,92 \\
\hline SEM-EDS & IGSR & $0.1-0.5 \mathrm{wt} \%$ & No & None & No & Yes & $\$ \$ \$ \$$ & hours & $\begin{array}{c}\text { micrometer } \\
\text { scale }\end{array}$ & $\begin{array}{c}\text { good- } \\
\text { excellent }\end{array}$ & Yes & $\begin{array}{c}\text { Yes - } \\
\text { submicron }\end{array}$ & 4,108 \\
\hline Raman & $\begin{array}{l}\text { Simultaneous } \\
\text { or Sequential }\end{array}$ & $\begin{array}{c}\text { single } \\
\text { particle sub- } \\
\text { ppb }\end{array}$ & No & $\begin{array}{l}\text { Depends on } \\
\text { collection }\end{array}$ & Yes & Some extent & $\$ \$-\$ \$ \$$ & minutes & $\begin{array}{l}\text { micrometer } \\
\text { scale }\end{array}$ & $\begin{array}{l}\text { good- } \\
\text { excellent }\end{array}$ & Some extent & $\begin{array}{c}\text { Yes - } \\
\text { submicron }\end{array}$ & $46,56,57$ \\
\hline $\begin{array}{c}\text { ICP-OES or } \\
\text { MS }\end{array}$ & IGSR & sub-ppb & Yes & $\begin{array}{c}\text { Yes, typically } \\
\text { digestion }\end{array}$ & No & Some extent & $\$ \$ \$$ & hours & bulk & excellent & No & No & $7,46,70$ \\
\hline GC-MS & OGSR & ppb-ppm & Yes & $\begin{array}{c}\text { Yes, } \\
\text { extraction } \\
\end{array}$ & No & Some extent & $\$ \$-\$ \$ \$$ & hours & bulk & excellent & No & No & 85 \\
\hline LC-MS & OGSR & ppb-ppm & Yes & $\begin{array}{c}\text { Yes, } \\
\text { extraction }\end{array}$ & No & Some extent & $\$ \$ \$-\$ \$ \$ \$$ & hours & bulk & excellent & No & No & 32,33 \\
\hline $\begin{array}{l}\text { MSI-TOF- } \\
\text { SIMS }\end{array}$ & Simultaneous & $\begin{array}{c}\text { single } \\
\text { particle, } \\
\text { qualitative }\end{array}$ & No & None & No & No & $\$ \$ \$ \$ \$$ & hours & $\begin{array}{l}\text { micrometer } \\
\text { scale }\end{array}$ & excellent & Yes & $\begin{array}{c}\text { Yes- } \\
\text { submicron }\end{array}$ & 90 \\
\hline LIBS & IGSR & low-ppm & Minimal & None & Yes & Some extent & $\$ \$$ & minutes & $\begin{array}{c}\text { micrometer } \\
\text { scale }\end{array}$ & $\begin{array}{c}\text { good- } \\
\text { excellent }\end{array}$ & No & Yes- micron & $\begin{array}{c}25,29,76 \\
113\end{array}$ \\
\hline $\begin{array}{c}\text { Electro- } \\
\text { chemistry }\end{array}$ & Simultaneous & ppb-ppm & $\begin{array}{l}\text { None to } \\
\text { minimal }\end{array}$ & $\begin{array}{l}\text { None to } \\
\text { minimal }\end{array}$ & Yes & Some extent & $\$$ & minutes & $\begin{array}{c}\text { bulk/ } \\
\text { micrometer } \\
\text { scale }\end{array}$ & $\begin{array}{l}\text { good- } \\
\text { excellent }\end{array}$ & No & No & 36 \\
\hline LA-ICP-MS & IGSR & sub-ppb & Minimal & None & No & Some extent & $\$ \$ \$$ & minutes & $\begin{array}{c}\text { micrometer } \\
\text { scale }\end{array}$ & excellent & No & Yes-micron & 72 \\
\hline
\end{tabular}




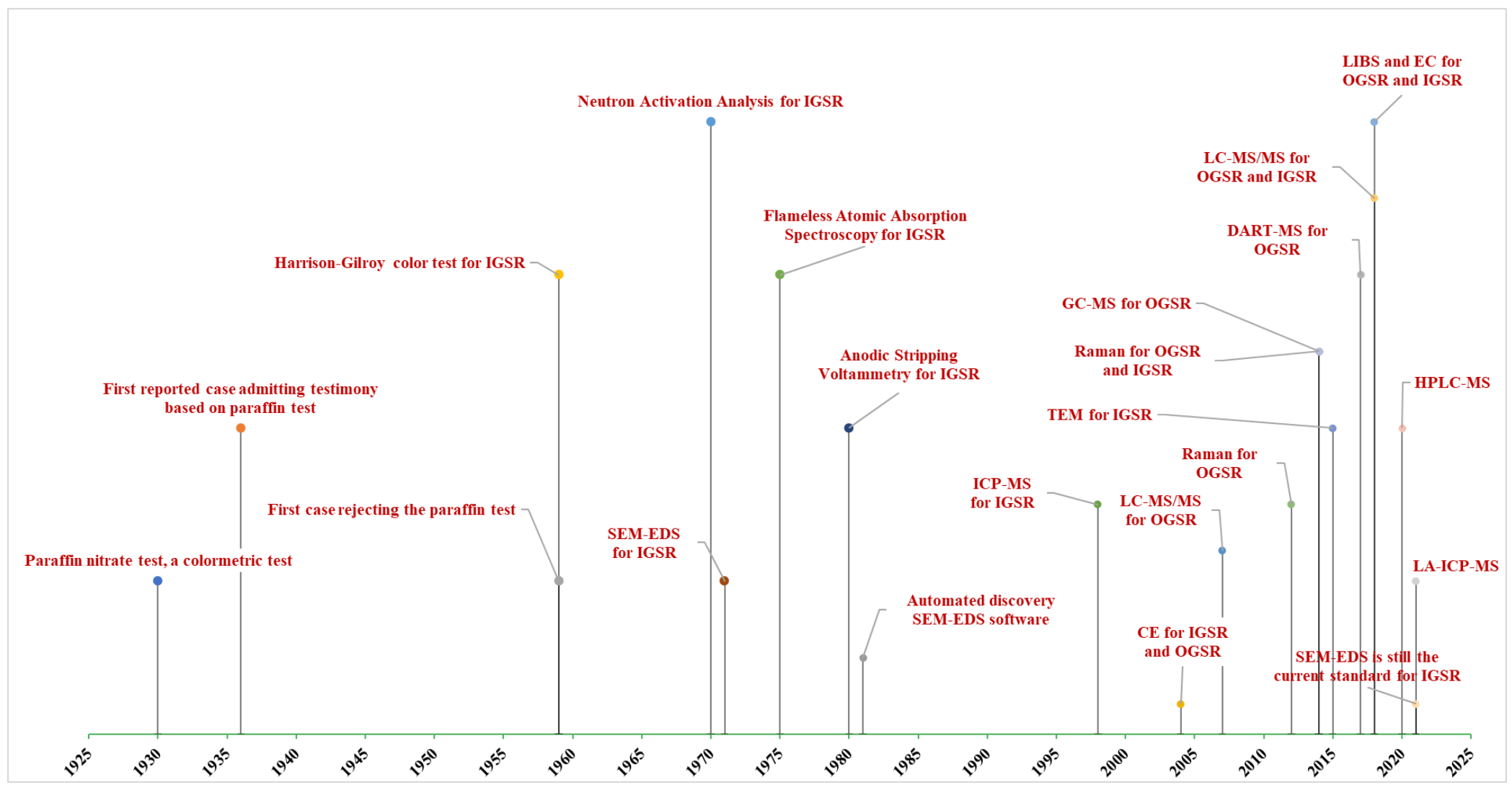

Figure 1: Historical Timeline of GSR analysis (adapted from Chang ${ }^{82}$, Hons ${ }^{69}$, and Giannelli ${ }^{167}$ )

12 


\subsubsection{Fundamentals of Project Instrumentation}

\subsubsection{Inductively Coupled Plasma-Mass Spectrometry (ICP-MS)}

Inductively coupled plasma (ICP) based techniques are attractive due to their high specificity and sensitivity along with the ability to detect trace elements. Two types of detection are commonly used with ICP ionization; MS and Optical Emission Spectrometry (OES), and both use the same mechanism of plasma creation, but different detection mechanisms. ${ }^{96,97}$ The typical instrumental components for ICP coupled to a mass spectrometer (MS) can be seen in Figure 2.A, alongside the other techniques of interest for this research project.

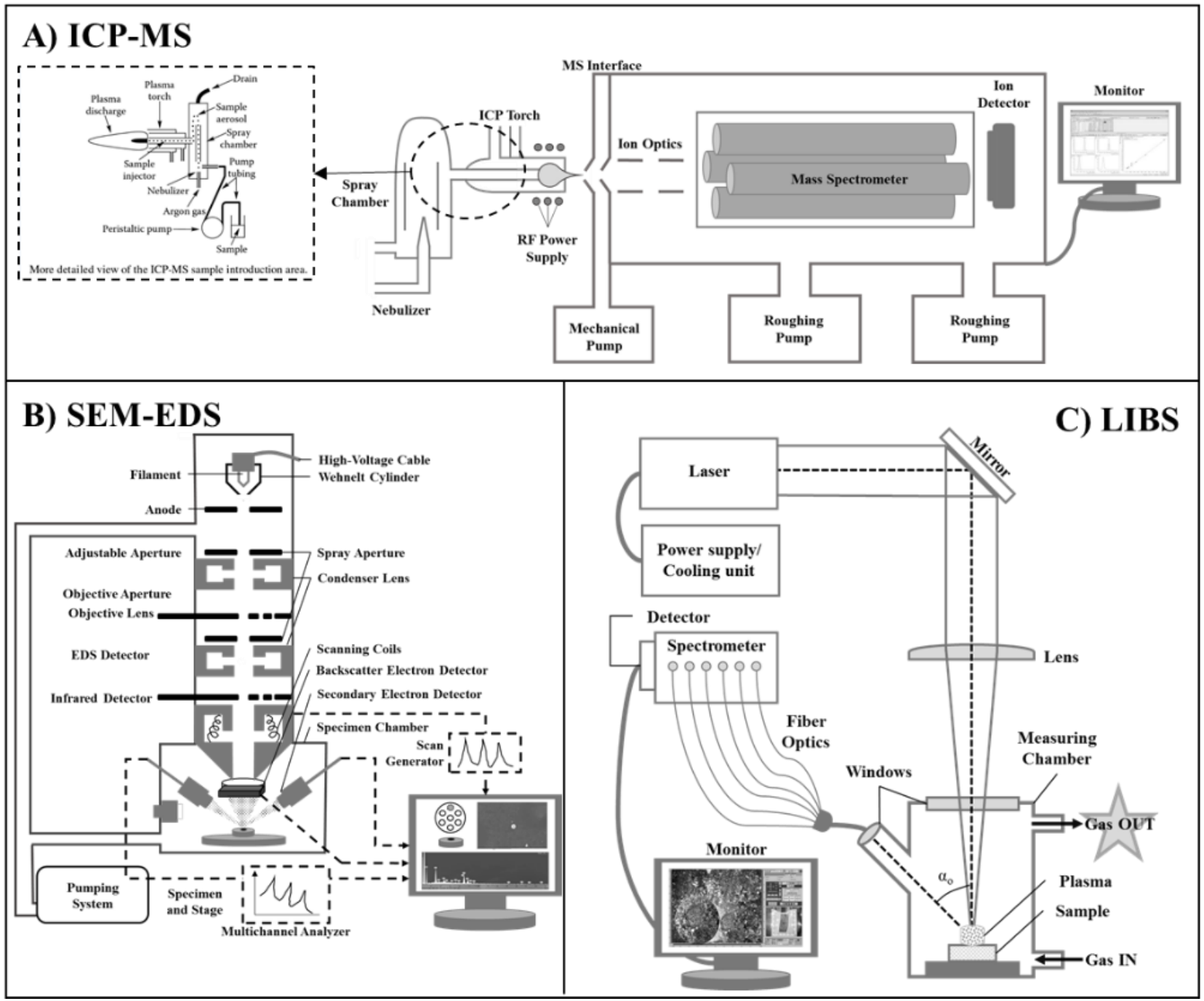

Figure 1: A) Schematic of a typical ICP-MS with a zoomed in schematic of the sample introduction area (more detailed view from 65) B) Schematic of the construction and working of various components located within the electron column and specimen chamber of the SEM C) Schematic representation of a typical LIBS setup. 
ICP is dependent upon a high-temperature plasma of around 6,000K-10,000 K produced using an argon gas flow, typically $15 \mathrm{~L} / \mathrm{min}$, and induced by a radio frequency running through a copper coil surrounding the plasma. Samples are introduced into the plasma in aerosolized formeither using a nebulizer and peristaltic pump for liquids or laser ablation for solids - the fine droplets are then atomized and ionized inside the plasma. ${ }^{97,98}$ ICP-OES depends on the ability of the detector to capture the emission of photons created by the excited species as the electrons fall back to ground state ${ }^{71,96,99}$ On the other hand, in ICP-MS, the ionized species are carried to a mass spectrometer and separated according to their mass-to-charge ratio by mass analyzer systems and then detected. ${ }^{99}$ MS provides superior selectivity than OES and detection limits often an order of magnitude lower. ${ }^{97}$ The main limitation of the application of these methods to GSR investigations is their destructive nature, as samples need to be digested with acid reagents and temperature prior analysis.

Despite the challenges, ICP-based techniques have been applied to the detection and quantitation of IGSR due to the high selectivity and sensitivity, with the added advantage of providing simultaneous analysis of multiple trace elements. ${ }^{68,70,100-102}$ Several studies conducted by Koons et. al. proved the utility of ICP based methods for the detection of IGSR, and even conducted a comparative study with AAS to demonstrate the differences in the techniques, and moving the field towards more use of ICP-OES. ${ }^{65,66,69,103}$ Then a more recent study conducted by Vanini et. al. optimized an ICP-OES method for the detection and quantification of $\mathrm{Pb}, \mathrm{Ba}$, and $\mathrm{Sb}$ in standard ammunition. ${ }^{70}$ Another study conducted by Costa et. al. applied ICP-mass spectrometry (ICP-MS) to the detection of multiple elements in nontoxic ammunition, including $\mathrm{Al}, \mathrm{Zn}$, and $\mathrm{Ti}^{7}$ While the use of ICP method is reliable, the samples require tedious preparation and consumption of the evidence without any morphological information. Laser Ablation-ICP-MS (LA-ICP-MS) has been proposed to overcome the sample preparation and consumption challenges, since a small area on the sample surface can be ablated and introduced as a dry-aerosol for elemental composition by ICP-MS. ${ }^{46,72,94,104,105}$

Solution-based ICP-MS has a place in research as an efficient technique, yet the technique lacks practical application to GSR casework. ICP techniques require the preparation of the sample in the form of digestion, making the technique time consuming and expensive. ${ }^{96,97}$ In forensic science, especially trace evidence, preserving as much of the sample for future analysis is an advantageous trait of a technique, especially since morphological information is still a critical part 
of GSR analysis. As a result, ICP-based methods are no longer used for GSR analysis in most forensic laboratories as they have been replaced by SEM-EDS. With the advent of modern laser ablation instruments, LA-ICP-MS has gained interest as the method is practically non-destructive and certain configurations can provide valuable spatial information and single particle analysis capabilities. ${ }^{46,72,94,104-106}$ The application is not yet mature enough to be adopted in the area of GSR but shows promise.

\subsubsection{Scanning Electron Microscopy- Energy Dispersive X-ray Spectroscopy (SEM-EDS)}

As an instrument, SEM-EDS is very versatile, and multiple types of detector are available depend on the purpose of analysis. A general design of a SEM-EDS instrument is shown in Figure 2.B. SEM instruments use a high-energy electron beam source created in a vacuum and finelyfocused in the column. This is accomplished by modifying and focusing the beam using apertures and various magnetic or electrostatic lenses, then precisely measuring an $\mathrm{x}-\mathrm{y}$ raster pattern in discrete locations on the sample to build a composite gray-scale image of the sample surface from the scan information. ${ }^{107} \mathrm{~A}$ schematic of information generated within the interaction volume of the electron beam and the sample can be seen in Figure 3. The image information of the discrete locations is contained within two types of electron products; lower kinetic energy secondary electrons (SEs) and higher incident energy backscatter electrons (BSEs). ${ }^{107,108}$ The energy difference between the two types of electrons is exploited in the analysis of IGSR. The higher atomic number elements will produce more BSEs, therefore the brightness of the image will indicate heavier elements, such as $\mathrm{Pb}, \mathrm{Ba}$, and $\mathrm{Sb}$, while low atomic number elements will appear darker in comparison. ${ }^{41,108}$ Subsequently, this improves the efficiency of automated detection since the heavier elements are easier to

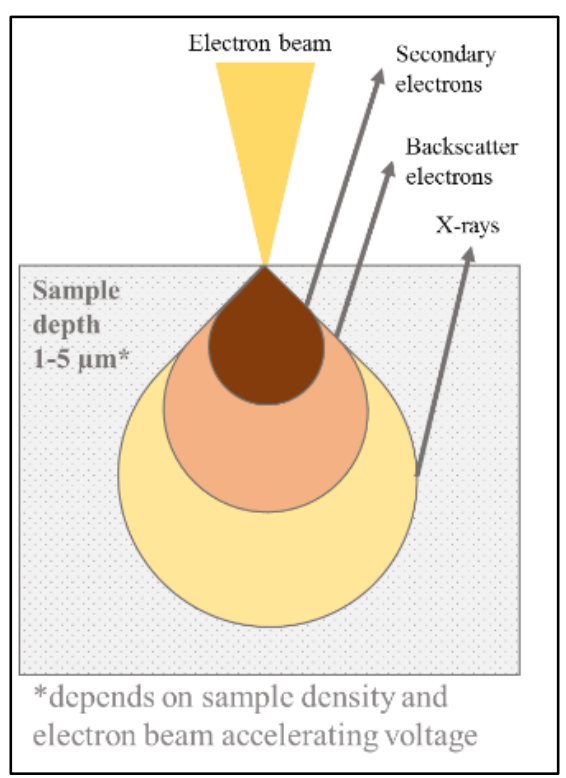

Figure 2: Diagram of the interaction volume for the electron beam and different types information emitted. detect based on brightness in the image. ${ }^{4}$

$\mathrm{X}$-rays are also produced as the electrons from the same beam interact with electrons in the sample through inelastic collisions, which cause the ejection of electrons from electronic shells. 
The movement of an electron leads to orbital transitions and subsequent characteristic discrete emission lines as they return to lower energy levels. ${ }^{108,109}$ Characteristic x-rays can be used to identify and quantify elements in a sample interaction volume ranging from $100 \mathrm{~nm}$ to $10 \mu \mathrm{m}$, depending on composition of the sample and beam energy. ${ }^{107}$ For the analysis of GSR, suspected particles are imaged and analyzed at magnification ranging from 500-10,000x, depending on the particle size. The electron beam is non-destructive to the IGSR, so once a spectrum is collected and corresponding particle(s) imaged, the sample can be stored for further testing or quality control.

Even though there is no sample preparation involved in the analysis of GSR stubs, the shortcomings of SEM-EDS is the high cost of the instrument and the extensive time to complete the analysis. ${ }^{90}$ There is also the emerging market of nontoxic ammunition with less heavy metals, which will require different parameters for accurate SEM-EDS detection. ${ }^{5}$ SEM-EDS has long been accepted by the forensic community and currently holds the standard for GSR analysis in crime laboratories due to its ability to simultaneously detect both elemental composition and the morphology of individual GSR particles, while preserving the evidence for future analysis. ${ }^{4,8,39-41}$ The run time on a sample can be several hours to days, even with current automation available. Then an analyst must review the morphology of the particles identified by the software, along with the spectrum collected, and compile their results and interpretation into a report. Depending of the number of items per case, the SEM-EDS data acquisition often takes days, while the whole process from submission to report take 29 to 105 days. ${ }^{110}$ Therefore, the criminal justice could benefit from complementary approaches that can reduce backlogs and improve response times.

\subsubsection{Laser Induced Breakdown Spectroscopy (LIBS)}

LIBS is an attractive alternative to the analysis of GSR because it provides a solution to some of the challenges faced by current instrumentation, such as speed of analysis, multi-elemental detection, and superior selectivity and sensitivity. A typical schematic for a LIBS instrument can be seen in Figure 2.C. A LIBS instrument focuses a laser beam into to a microscopic area ranging from 4-200 $\mu \mathrm{m}$ diameter, and the high energy concentrated in a small space establishes a high irradiance (power per unit area) that breaks down the molecules within the sample. The laserinduced breakdown process occurs when irradiances in the order of $10^{9}-10^{10} \mathrm{~W} / \mathrm{cm}^{2}$ are reached. A temporal plasma is then created that reaches temperatures of over $8,000 \mathrm{~K}$, causing the sample 
to vaporize and the excitation of ions, atoms and molecules. ${ }^{111}$ As the temporal plasma relaxes, the excited species within the plasma return to ground states, producing the emission of radiation at specific wavelengths for the elements present in the sample. ${ }^{112}$ The element-specific emissions are rapidly collected and dispersed by a spectrograph to obtain a spectrum of the sample's elemental composition in terms of intensities as a function of wavelength; the most common type being a Czerny-Turner for the ability to perform simultaneous detection with high spectral resolution as low as $0.03 \mathrm{~nm}$, depending on the spectrometer and detector combination. ${ }^{112}$ The output is measured by a detector, such as a charge coupled device or an intensified charge coupled device, and provides spectral composition by interpreting the radiation emitted by the plasma. ${ }^{111}$ An ideal spectrometer-detection system is one that has wide wavelength range, high spectral resolution, wide dynamic range, while still capable of short readout and data acquisition time. ${ }^{111}$

In addition, LIBS technology offers field portable applicability, and is a growing intrest in numerous disciplines, especially the environmental and forensic sciences, since there is no sample preparation involved which is ideal for field work. ${ }^{76,113-116}$ Doña-Fernández et. al. performed a comparison between current SEM-EDS technology and a portable LIBS using 135 samples collected from shooters, non-shooters, and the clothing of police officers. The portable LIBS instrument was found to have over $90 \%$ accuracy of classification into the groups of shooter and non-shooter. ${ }^{76}$ Additional studies have shown LIBS and its ability to provide spatial information using different ablation patterns in combination with the multielemental information provided. ${ }^{24-}$ ${ }^{26}$ The results of these studies demonstrate the promise of a technique such as LIBS to strengthen the value of GSR evidence using accurate and rapid multielement analysis with instruments that can be operated in the laboratory or on-site. ${ }^{22,23,25,29,76}$

\subsubsection{Detection Methods for OGSR}

OGSR has recently become of interest to the forensic community due to the additional value for the interpretation of GSR evidence. ${ }^{21,34,89}$ Tarifa et. al. examined Capillary Microextraction of Volatiles (CMV) for headspace extraction of volatile OGSR compounds, and then thermally desorbing the compounds from the capillary into a GC-MS injector. ${ }^{85}$ Another group utilized liquid chromatography-quadrupole time-of flight mass spectrometry (LC-QTOF) to identify 17 analytes - including, but not limited to MC, EC, DPA, 2,4-DNT — associated with OGSR. Liquid extraction was conducted from both swabs and tape lifts at concentrations in the 
ppb range. ${ }^{32}$ To overcome the issue of sample preparation, thermal desorption techniques, such as IMS, have been successfully used as screening techniques for OGSR and explosives. ${ }^{35,61,86,92}$ Research on the use of these techniques is important for understanding the nature of OGSR, yet destruction of the evidence, and lack of morphology leave these types of methods at a disadvantage for a confirmatory technique. Infrared techniques, such as Fourier Transform Infrared Spectroscopy (FTIR) or Raman Spectroscopy, have also been investigated for OGSR detection. Studies have successfully identified major compounds associated with OGSR, such as DPA and EC. ${ }^{48,56,57,83,117}$ An advantage of these methods is no sample preparation involved, but consideration must be taken if the adhesive background is a dark color.

\subsubsection{Simultaneous or Sequential Detection Methods for the Identification of OGSR and IGSR}

Electrospray Trapped Ion Mobility Mass Spectrometry (ESI-TIMS-MS) studied by McKenzie-Coe et. al. is an example of a sequential method which forms organometallic complexes followed by fast, post-ionization, high resolution mobility and mass separations. ${ }^{61}$ The use of organometallic complexes and the transfer of volatile materials into the gas phase make an TIMS technique able to detect inorganic elements and organic compounds associated with GSR ${ }^{61}$ While the technique has the advantage of sequential detection, the disadvantage is the requirement of the sample preparation using capillary electrophoresis, along with the time and equipment cost necessary for the analysis. Raman has also been recently reported as a sequential technique for the detection of OGSR in combination with cathodoluminescence, X-ray diffraction (XRD), and EDS. ${ }^{48,118}$ A study conducted by Bueno and Lednev successfully mapped the presence of OGSR particles, identified the inorganic elements in GSR, and differentiated from the tape background, determining it provided no interference to the sample. ${ }^{57}$

A simultaneous technique utilizing sub-micrometric Mass Spectrometry Imaging- Time of Flight- Secondary Ion Mass Spectrometry (MSI-TOF-SIMS) can simultaneously detect IGSR and OGSR by building composite images with both the organic and inorganic information of a sample, including the morphology of the particle on a micrometer scale. ${ }^{90}$ The process of building the image requires the use of Principal Component Analysis (PCA). The image is combined using the first two principal components to display information about the spatial placement of the IGSR and OGSR on the surface of the sample, and subsequently the morphology. 
Another very appealing technique is electrochemistry which can complete analysis in the matter of minutes and detect simultaneously multiple inorganic elements and organic compounds of interest with minimal sample preparation during a single voltametric run. ${ }^{36,62}$ The electrochemistry involves the induced reduction and oxidation of a sample while applying a range of voltages, and is able to identify analytes based on their peak potential which is displayed by a voltammogram. ${ }^{23,36}$ Electrochemistry has the advantage of high sensitivity and specificity on small amounts of samples at low concentrations, while also being complementary to SEM-EDS confirmatory analysis. ${ }^{23,62,119}$ The technique being develop in our laboratory allows the sample to be collected directly from a typical GSR stub, and identifies elements such as $\mathrm{Pb}, \mathrm{Cu}$, and $\mathrm{Sb}$ along with compounds such as 2,4-DNT, DPA and NG, while leaving a majority of the stub untouched for additional analysis. ${ }^{23,36}$

Lastly, mass spectrometry (MS) is a technique that can be coupled to different ionization sources and separation techniques, providing high sensitivity and specificity for OGSR compounds of interest. While MS is typically considered a "confirmatory" method in most forensic disciplines, the mere confirmation of organic (i.e., by chromatographic separation using GC or LC coupled to a MS) or inorganic species (i.e., by ICP-MS) does not provide unambiguous identification of gunshot residues since many compounds associated with OGSR and IGSR are also present in the environment. ${ }^{33,120}$ Instead, the strongest support for the identification of GSR is provided by the combined characteristics of particle's morphology and chemical composition by SEM-EDS, or by providing combined evidence of the presence of IGSR and OGSR on the same sample.

\subsection{Transfer and Persistence of GSR}

Understanding the importance of transfer is essential to the interpretation of any trace evidence, and GSR is no exception since the evidence is exposed to both secondary and tertiary transfer. ${ }^{42,54,121-128}$ The particles created during discharge are micrometers in size, making contaminated surfaces difficult to identify and particle transfer imperceptible. The GSR particles can be dislodged and transferred depending on the post-shooting activity of an individual, or if something comes into contact with a contaminated surface, such as physical contact with someone who was recently near a discharged firearm or a surface where a firearm was placed. ${ }^{54,123}$ Therefore, including locations, professions, and hobbies where someone might come into contact with these sources of contamination are essential in population studies. 
If an individual is close to the discharging of a firearm, handles the spent cartridge cases, or conceals the weapon prior to being sampled, then particles of IGSR are likely to be physically displaced onto the individual. ${ }^{54,129}$ Factors, such as distance from the firing event, the weapon involved, the type of ammunition used, or type of clothing, can also affect the transfer of GSR, and in turn, these can also introduce variation in the GSR evidence recovered. ${ }^{32,82,130-135} \mathrm{~A}$ study conducted by Brozek-Mucha counted the number of particles at different shooting vicinities, and on different substrates, to examine their chemical composition and size. The larger particles consisted of $\mathrm{Sb}$ and $\mathrm{Sn}$ and traveled further down range from the point of discharge, while smaller characteristics particles of $\mathrm{Pb}, \mathrm{Ba}$, and again $\mathrm{Sb}$, did not travel as far due to less mass and more resistance. ${ }^{136}$ The trend of particle sizes held for both leather and cotton samples, but the leather samples contained less GSR particles than its matched pair cotton sample. A different study by French and Morgan saw that the GSR deposited on an individual in the vicinity of a firearm discharge could also transfer dozens of particles (21-51 particles) to another person-secondary transfer - and that person could transfer between 12-22 of those particles to another persontertiary transfer- through as little contact as a handshake. ${ }^{123}$

The previous studies only examined IGSR, and the particles susceptibility to deposition on surfaces during firearm discharge process. Recently, studies into the transfer of OGSR have been also conducted. A study by Hofstetter et. al. examined the amount of OGSR deposited on the clothing and hands of control-shooters. ${ }^{21}$ In the study, OGSR was found in higher concentrations on the clothing than the hands of control-shooters, and this was attributed to the fibers trapping the compounds, but secondary transfer of OGSR between individuals was not as common. ${ }^{21,124,135,137}$ However, the nature of OGSR makes the evidence susceptible to evaporation and absorption since its comprised of volatile and reactive compounds. ${ }^{37,61}$ This causes a short-lived deposit of evidence, only ranging from a couple hours to at a maximum a day before OGSR compound of interest are lost.

Multiple studies have been conducted by Andrasko, et. al., on the persistence of different types of ammunitions used in shotguns, rifles, pistols, and revolvers. Typical hand samples were collected, along with direct sampling from the recently discharged firearm, or spent cartridge cases, to determine the persistence GSR evidence on different surfaces of the firearm evidence. ${ }^{131-}$ 134 The researchers utilized Solid Phase Micro-Extraction (SPME) for the detection of volatile chemicals in the headspace of cartridges to determine combustion products, mainly types of 
OGSR, such as naphthalene and nitroglycerin. The study supported the conclusion that there is a loss of OGSR over time, due to their volatile nature. Determination of an exact time of loss is challenging due to many factors involved during the discharge of a firearm - environmental factors and different industry combinations of primer and propellant compounds for different end use purposes. ${ }^{131,133,134}$ Still, the multipart study examined the loss of OGSR while awaiting examination, and the results showed that within 6 days, the volatiles of interest evaporated. ${ }^{132}$ Multiple studies have determined the stability of OGSR on collection stubs to be to up to approximately 15 days for most compounds of interest, if stored at $4^{\circ} \mathrm{C}$ and in the dark as soon as collected. ${ }^{37,61,90,137}$ The extreme differences in the stability OGSR and IGSR can add information, but the rapid loss of OGSR causes time between firing, collection, and analysis to be critical for preservation of evidence. Preservation of GSR in its entirety is important for interpretation and reporting of the evidence.

\subsection{High-Risk Background Populations}

Components related to GSR, both organic and inorganic, are common in the environment and can cause false positives during analysis. ${ }^{30,37,40,41,138}$ Numerous activities, hobbies, and professions can create substances which can be mistaken for GSR or with a firearm discharge, such as mechanics, pyrotechnicians, and security officers. ${ }^{22,125,138,139}$ Other populations for sources that mimic GSR includes electricians, painters, percussion cap operated tools, and even individuals who handle paper. ${ }^{7,22,24,29,46,105,140}$ The research purposed by this project includes as many highrisk activities and occupations as possible to expand the current body of knowledge and our understanding of the influence these populations have on the interpretation of GSR evidence.

A subpopulation often studied because of the propensity to cause false positive results for IGSR are mechanics, specifically people that handle brake pads, which contain barium sulfate as a heat stabilizer and antimony sulfide as a solid lubricant. ${ }^{22,24,46,105,139,140}$ In a study by Tucker et. al. 75 different brake pads were collected and examined by SEM-EDS. ${ }^{139}$ Eleven different subject's hands were sampled, and over 115,000 particles were collected from individuals who handled the brake pads. Combinations of elements consistent with IGSR were identified, along with other elements such as titanium, copper, and zinc. ${ }^{4}$ The study did not find any characteristic particles of IGSR but over 600 consistent particles. Further, the particles imaged did not possess the spherical shape, and the study suggested the lack of shape was due to the difference in 
temperature and pressure during formation. ${ }^{139}$ The confirmation of additional elements present in the composition of brake pads is especially important for this purposed project, since nontoxic GSR may have elemental compositions that overlap, such as iron, titanium, tin, and strontium.

Another form of false positives associated with automobiles are the particles created when some airbags deploy during a collision and the airbag system uses a mechanism that is triggered by a solid propellent very similar to a GSR primer. ${ }^{138,141}$ The use of a primer provides a primary explosive to trigger a controlled explosion that rapidly deploys the airbags. Even though there are filters in place to prevent the particle/ gas mixture from escaping, particles typically eject during the process and are transferred onto individuals during wreck and any subsequent on-site medical personnel. A two-part study conducted by Robert Berk in Illinois first examined airbag debris for particle shape and composition. The study collected samples from inside a deployed airbag and analyzed them by SEM-EDS. On average, each stub contained almost 1600 particles containing elements found in GSR, with the majority possessing iron, zinc, and copper, along with minor elements being zirconium, aluminum, and strontium. During SEM-EDS analysis, numerous spherical particles were imaged, and the corresponding spectrum contained combinations of elements that could be mistaken for GSR. ${ }^{138}$ The second part of the study collected samples from different model cars, and came to the conclusion that airbags deployed with primer cap systems can create particles similar to GSR. ${ }^{141}$ The deployment of an airbag is a larger, complex process with many other materials involved, such as different gases for the inflation of a fiber based cushion.

Another study by Grima et. al. examined the airborne discharge produced by a firework's display, something very common all over the world. ${ }^{142}$ Carbon stubs were placed in the area surrounding the display and collected the airborne debris and residues as the fireworks were discharged, in addition to one hair sample. This design was an attempt to gauge the exposure of technicians and audience members during a firework display. The study found particles containing elements commonly found in IGSR, and 5 particles that were indistinguishable from IGSR in both elemental composition and morphology according to criteria of the ASTM standard. ${ }^{142}$ There is a possibility some particles could have mimicked nontoxic GSR for two reasons; 1) many of the same elements are present in both nontoxic ammunition and fireworks (for example, concussion powders and zinc), and 2) fireworks are the ideal combination of temperature and pressure to create the micrometer particles examined for IGSR. 
There is also concern for the secondary transfer of GSR by individuals who regularly handle firearms. Studies have researched the transfer of GSR by police officers, since they have an occupation where a firearm is typically handled on every shift. ${ }^{42,54,122,125,135,143-145}$ As a result, precautions should be taken if a police officer should collect GSR evidence from a suspect or transport them in their vehicle. A study conducted by Hannigan et. al. sampled police officer's clothing and found 131 particles on the cuffs of 98 garments. Of the particles discovered, only 3 were characteristic of GSR, but over 100 were consistent with GSR. ${ }^{42}$ Another study examined the hands of officers following the start of their shift, immediately following the first handling of their firearm. ${ }^{54}$ Thirty-three officer's hand were sampled and the number of characteristic GSR particles ranged from none on some officers to hundreds on others. As for consistent GSR particles, all the sampled hands had at least one, while others had hundreds. From this study, it was concluded that by the beginning of their shift, these officers already had possible sources of contamination on them.

\subsection{Statistical Analysis of Gunshot Residue}

The numerous challenges in the analysis and interpretation of GSR evidence have been demonstrated by the discussion of multiple external factors producing the elemental composition and morphology similar to GSR, along with the prevalence of actual GSR on the hands of individuals not involved in criminal events. As a result, bigger picture questions need to be answered through population studies and statistical analysis. Inclusion of large background populations in statistical models are becoming an essential area of forensic research. A variety of statistical approaches have been studied to enhance objectivity on decision thresholds, such as principal component analysis, ${ }^{8}$ machine learning algorithms, ${ }^{146}$ Bayesian approaches, ${ }^{130,147}$ and likelihood ratios. ${ }^{6,45,82,120,130,147,148}$ An aim of this study is to provide a large dataset that includes broader subpopulations, to validate overarching statistical methods to strengthen evidence interpretation and scientific validity of GSR.

Principal Component Analysis (PCA) is a powerful visualization technique for examining the natural clustering and inherent variability of a data set, offering support for the further study of classification and prediction techniques. ${ }^{890} \mathrm{PCA}$ is unsupervised, meaning the original classification of the data is unknown to the model, and any separation in the groups is based purely on the variability within the data. A study conducted by Castellanos et. al. performed PCA on 
samples from the hands of control-shooters using Mass Spectrometry Imaging Time of FlightSecondary Ion Mass Spectrometry (MSI TOF-SIMS). ${ }^{90}$ PCA allowed for the chemical mapping of both IGSR and OGSR due to the inherent separation of PC1 being influenced by the presence of OGSR, and PC2 being influenced by the presence of IGSR. ${ }^{90}$ Another study by Hogg et. al. examined six brands of nontoxic ammunition, and a road flare, and then conducted PCA on the SEM-EDS data. PCA showed clustering of the different manufactured ammunition, but only after all the spectra obtained from a single firing event were truncated and averaged to help overcome the variability of ammunition's composition. ${ }^{8}$ This research laid the framework for exploratory data analysis that can be further used for classification and prediction models.

Multivariate techniques are appealing for GSR analysis due to the variety of elements and variable concentrations found in different manufactured primers, and the resulting GSR ${ }^{87,149,150} \mathrm{~A}$ type of multivariate technique is Linear Discriminant Analysis (LDA), and it is advantageous because the model receives supervised training using data where the ground truth of the sample classes are known, and allowing for the subsequent estimation of the model's performance measures. LDA aims to maximize the separation between the known classes using the training dataset, and then provides prediction of unknown samples inputted into the model through testing and validation datasets. ${ }^{151}$ LDA is one of the many applications where discriminant analysis is applied, and they are widely available and commonly applied to scientific data analysis. ${ }^{8,45,152}$

One type of well-documented, supervised, prediction algorithm is Logistic Regression (LR) and had been applied to the classification of GSR. ${ }^{153}$ The method can be used to classify binary outcomes, such as the presence of GSR versus the absence of GSR. LR determines for the optimal decision boundary to best separate the classes using supervised training and the ground truth knowledge of the data's class. A logistic function works to maximize the likelihood of observing a set of sample values, then adjusts the linear separation based on this observation. In addition, LR transforms the prediction to a value between 0 and 1 and models the probability of class membership, leading to the ability to build likelihood ratios from the probabilites. ${ }^{154}$ Another strength of LR is the wide acceptance of the algorithm in the scientific community.

Naïve Bayes (NB) is also another widely used algorithm and provides probabilities as on output. While NB and LR are similar in they are both supervised, prediction algorithms, NB is based on Bayes Theorem and determines probability of a given sample values being associated with a class. This is possible because NB assumes that the predictor variables are independent, 
although this is not always the case. The assumption is practical since it reduces multidimensional tasks, simplifies classification, and allows the class conditional to be calculated separately for each variable, the training data set can be small, and the predictions can be scaled. ${ }^{155}$ All of these features make NB an attractive prediction algorithm.

Another predictive approach is Neural Networks (NN). ${ }^{20,146,151}$ Much like the name implies, the training process of neural networks is akin to the biological process of the neurons in the brain, but on a much more simplistic level. ${ }^{156}$ A major advantage to neural networks is the ability of the training algorithm to respond to small changes within the data environment. ${ }^{157}$ These types of classification techniques have been successfully applied for decades in the food industry, and more recently to the analysis of GSR. ${ }^{25,36,146,156}$ This research will apply a similar model previously validated at WVU for the interpretation of OGSR, ${ }^{146}$ using backpropagation neural networks. The outputs are used to create decision thresholds to perform probability density modeling, provide the probability of a sample belonging to a specific group, and then give the ability to calculate likelihood ratios for evidence interpretation. ${ }^{130}$

Probabilistic methods have been proposed for applying a more intuitive approach to evidence interpretation, therefore making the presentation in court simpler to explain and represent. Explaining the strengths and weaknesses of evidence to any criminal justice system stakeholder is challenging, but these probabilities can assist to support the analyst's decision making process in a more understandable way. ${ }^{158-160}$ A common metric presented to stakeholders, such as judges and lawyers, is the likelihood ratio. Forensic experts sometimes implement the use of likelihood ratios to support the weight of the evidence, and then the tier of fact reaches the final conclusion, called the posterior odds. ${ }^{130,146-148,161-163}$ The value of the likelihood ratio from the expert, along with any additional information that may have been withheld from the expert to avoid bias during analysis, is applied to reach a final conclusion. An additional advantage of a Bayesian likelihood ratio approach is the ability to consider multiple variables and circumstances in the decision process, not only the answer to the question of if the evidence collected is in fact GSR.

Bayesian Networks (BN) is another approach recently explored for GSR interpretation. Bayesian networks become a popular tool in the evaluation of multiple variables and relationships between the evidence and the circumstances under which the evidence was collected. $^{20,130,147,148,164,165}$ Variables that can be considered during the analysis of GSR are the background content on the person's hands, the time since the shooting event, various activities, 
combination of OGSR and IGSR, the number of particles recovered, and other possible environmental conditions. ${ }^{130,147,148,164,165}$ The Bayesian network takes into account multiple interacting variables and sources of uncertainty, to provide a final assessment of the value of the evidence as to whether it is or is not GSR, depending on the output of the network after considering all the variables. ${ }^{87}$ It also has the advantage to include aspects of transfer and persistence, also known as activity factors. The proposed study aims to expand these probabilistic models mentioned to larger populations, including those in contact with nontoxic primers and individuals with anticipated high-risk backgrounds.

\subsection{References}

(1) National Crime Victimization Survey https://www.bjs.gov/index.cfm?ty=nvat (accessed $\operatorname{Mar} 4$, 2021).

(2) Burch, A. M.; Durose, M. R.; Walsh, K. A.; Tiry, E.; Statistics, B. of J. Publicly Funded Forensic Crime Laboratories: Resources and Services, 2014; 2016.

(3) Durose, M. R.; Walsh, K. A.; Burch, A. M. Census of Publicly Funded Forensic Crime Laboratories, 2009.; Bureau of Justice Statistics, 2012.

(4) Standard Guide for Gunshot Residue Analysis by Scanning Electron Microscopy / Energy Dispersive X-Ray Spectrometry 1; West Conshohocken, PA, 2009. https://doi.org/10.1520/E1588-20.2.

(5) Brozek-Mucha, Z. Trends in Analysis of Gunshot Residue for Forensic Purposes. Anal. Bioanal. Chem. 2017, 409, 5803-5811.

(6) Fambro, L. A.; Miller, E. T.; Vanderbos, D. D. Characterization of Lead-Free Gunshot Residue Analogs. Anal. Methods 2016, 8, 3132-3139. https://doi.org/10.1039/c6ay00725b.

(7) Costa, R. A.; Motta, L. C.; Destefani, C. A.; Rodrigues, R. R. T.; Santo, K. S. D.; Aquije, G.; Boldrini, R.; Athayde, G. P. B.; Carneiro, M.; Romao, W. Gunshot Residues (GSR) Analysis of Clean Range Ammunition Using SEM/EDX, Colorimetric Test and ICP-MS: A Comparative Approach between the Analytical Techniques. Microchem. J. 2016, 129, 339-347. https://doi.org/10.1016/j.microc.2016.07.017.

(8) Hogg, S. R.; Hunter, B. C.; Smith, R. W. Elemental Characterization and Discrimination of Nontoxic Ammunition Using Scanning Electron Microscopy with Energy Dispersive 
X-Ray Analysis and Principal Components Analysis. J. Forensic Sci. 2016, 61 (1), 35-42. https://doi.org/10.1111/1556-4029.12881.

(9) Donghi, M.; Mason, K.; Romolo, F. S. Detecting Gunshot Residue from Sellier \& Bellot Nontox Heavy Metal-Free Primer by in Situ Cathodoluminescence. J. Forensic Sci. 2019, 64 (6), 1658-1667. https://doi.org/10.1111/1556-4029.14110.

(10) Romano, S.; De-Giorgio, F.; D’Onofrio, C.; Gravina, L.; Abate, S.; Romolo, F. S.

Characterisation of Gunshot Residues from Non-Toxic Ammunition and Their Persistence on the Shooter's Hands. Int. J. Legal Med. 2020, 134 (3), 1083-1094.

https://doi.org/10.1007/s00414-020-02261-9.

(11) Lowenkamp, C. T.; VanNostrand, M.; Holisinger, A. The Hidden Costs of Pretrial Detention; Arnold Foundation, 2013.

(12) Pretrial Justice: How Much Does It Cost?; Pretrial Justice Institute, 2017.

(13) Henrichson, C.; Rinaldi, J.; Delaney, R.; Justice, V. I. of. The Price of Jails: Measuring the Taxpayer Cost of Local Incarceration; 2015.

(14) Gold, R. M. Jail as Injunction LK - Https://Libwvu.on.Worldcat.Org/Oclc/8260064021. Georg. LAW J. TA - TT - 2019, 107 (3), 501-560.

(15) Ostrom, B. J.; Hanson, R. A. Efficiency, Timeliness, Quality: A New Perspective from Nine State Criminal Trial Courts; National Center for State Courts, 1999.

(16) McCollister, K. E.; French, M. T.; Fang, H. The Cost of Crime to Society: New CrimeSpecific Estimates for Policy and Program Evaluation. Drug Alcohol Depend. 2010, 108, 98-109.

(17) Lydersen, K. Wrongful Conviction Costs Keep Climbing. 2013.

(18) S.B., B. Costs of Pretrial Detention LK Https://Libwvu.on.Worldcat.Org/Oclc/7065880635. Bost. Univ. Law Rev. TA - TT 2017, 97 (1), 1-30.

(19) Maitre, M.; Horder, M.; Kirkbride, K. P.; Gassner, A.-L.; Weyermann, C.; Roux, C.; Beavis, A. A Forensic Investigation on the Persistence of Organic Gunshot Residues. Forensic Sci. Int. 2018, 292, 1-10. https://doi.org/10.1016/j.forsciint.2018.08.036.

(20) Maitre, M.; Kirkbride, K. P.; Horder, M.; Roux, C.; Beavis, A. Current Perspectives in the Interpretation of Gunshot Residues in Forensic Science: A Review. Forensic Sci. Int. 2017, 270, 1-11. 
(21) Hofstetter, C.; Maitre, M.; Beavis, A.; Roux, C. P.; Weyermann, C.; Gassner, A.-L. A Study of Transfer and Prevalence of Organic Gunshot Residues. Forensic Sci. Int. 2017, 277, 241-251. https://doi.org/https://doi.org/10.1016/j.forsciint.2017.06.013.

(22) Dockery, C. R.; Goode, S. R. Laser-Induced Breakdown Spectroscopy for the Detection of Gunshot Residues on the Hands of a Shooter. Appl. Opt. 2003, 42 (30), 6153-6158. https://doi.org/10.1364/ao.42.006153.

(23) Trejos, T.; Vander Pyl, C.; Menking-Hoggatt, K.; Alvarado, A. L.; Arroyo, L. E. Fast Identification of Inorganic and Organic Gunshot Residues by LIBS and Electrochemical Methods . Forensic Chem. 2018, 8, 146-156. https://doi.org/doi.org/10.1016/j.forc.2018.02.006.

(24) Lopez-Lopez, M.; Alvarez-Llamas, C.; Pisonero, J.; Garcia-Ruiz, C.; Bordel, N. An Exploratory Study of the Potential of LIBS for Visualizing Gunshot Residue Patterns. Forensic Sci. Int. 2017, 273, 124-131. https://doi.org/10.1016/j.forsciint.2017.02.012.

(25) Menking-Hoggatt, K.; Arroyo, L.; Curran, J.; Trejos, T. Novel LIBS Method for MicroSpatial Chemical Analysis of Inorganic Gunshot Residue. J. Chemom. 2019, e3208, 13. Pyl, C. Vander; Ovide, O.; Ho, M.; Yuksel, B.; Trejos, T. Spectrochemical Mapping Using Laser Induced Breakdown Spectroscopy as a More Objective Approach to Shooting Distance Determination. Spectrochim. Acta Part B At. Spectrosc. 2019, 152, 93-101. https://doi.org/https://doi.org/10.1016/j.sab.2018.12.010.

(27) Vander Pyl, C.; Morris, K.; Arroyo, L.; Trejos, T. Assessing the Utility of LIBS in the Reconstruction of Firearm Related Incidents. Forensic Chem. 2020, 19 (May), 100251. https://doi.org/10.1016/j.forc.2020.100251.

(28) Fambro, L. A.; Vandenbos, D. D.; Rosenberg, M. B.; Dockery, C. R. Laser-Induced Breakdown Spectroscopy for the Rapid Characterization of Lead-Free Gunshot Residues. Appl. Spectrosc. 2017, 71 (4), 699-708. https://doi.org/10.1177/0003702816689099. Silva, M. J.; Cortez, J.; Pasquini, C.; Honorato, R. S.; Paima, A. P. S.; Pimentel, M. F.; Paim, A. P. S.; Pimentel, M. F. Gunshot Residues: Screening Analysis by Laser-Induced Breakdown Spectroscopy. J. Braz. Chem. Soc. 2009, 20 (10), 1887-1894. https://doi.org/10.1590/S0103-50532009001000017.

(30) Rosenberg, M. B.; Dockery, C. R. Determining the Lifetime of Detectable Amounts of Gunshot Residue on the Hands of a Shooter Using Laser-Induced Breakdown 
Spectroscopy. Appl. Spectrosc. 2008, 62 (11), 1238-1241.

https://doi.org/10.1366/000370208786401473.

(31) Schwoeble, A. J.; Strohmeier, B. R.; Piasecki, J. D.; Spie, N. I. S. The Influence of Surface Chemistry on GSR Particles: Using XPS to Complement SEM/EDS Analytical Techniques. In Conference on Scanning Microscopy; Technol, Ed.; Monterey, CA, 2010; Vol. 7729. https://doi.org/10.1117/12.863906.

(32) Benito, S.; Abrego, Z.; Sánchez, A.; Unceta, N.; Goicolea, M. A.; Barrio, R. J. Characterization of Organic Gunshot Residues in Lead-Free Ammunition Using a New Sample Collection Device for Liquid Chromatography-Quadrupole Time-of-Flight Mass Spectrometry. For. Sci. Int. 2015, 246 (79-85).

(33) Taudte, R. V; Roux, C.; Blanes, L.; Horder, M.; Kirkbride, K. P.; Beavis, A. The Development and Comparison of Collection Techniques for Inorganic and Organic Gunshot Residues. Anal. Bioanal. Chem. 2016, 408 (10), 2567-2576. https://doi.org/10.1007/s00216-016-9357-7.

(34) Feeney, W.; Vander Pyl, C.; Bell, S.; Trejos, T. Trends in Composition, Collection, Persistence, and Analysis of IGSR and OGSR: A Review. Forensic Chem. 2020, 19. https://doi.org/10.1016/j.forc.2020.100250.

(35) Joshi, M.; Delgado, Y.; Guerra, P.; Lai, H.; Almirall, J. R. Detection of Odor Signatures of Smokeless Powders Using Solid Phase Microextraction Coupled to an Ion Mobility Spectrometer. Forensic Sci. Int. 2009, 188 (1), 112-118. https://doi.org/https://doi.org/10.1016/j.forsciint.2009.03.032.

Ott, C. E.; Dalzell, K. A.; Calderon-Arce, P. J.; Alvarado-Gamez, A. L.; Trejos, T.; Arroyo, L. E.; Calderón-Arce, P. J.; Alvarado-Gámez, A. L.; Trejos, T.; Arroyo, L. E. Evaluation of the Simultaneous Analysis of Organic and Inorganic Gunshot Residues Within a Large Population Data Set Using Electrochemical Sensors*(,)Dagger. J. Forensic Sci. 2020, 65 (6), 1935-1944. https://doi.org/10.1111/1556-4029.14548.

(37) Taudte, R. V; Roux, C.; Beavis, A. Stability of Smokeless Powder Compounds on Collection Devices. For. Sci. Int. 2017, 270, 55-60.

(38) Goudsmits, E.; Sharples, G. P.; Birkett, J. W. Preliminary Classification of Characteristic Organic Gunshot Residue Compounds. Sci. Justice 2016, 56 (6), 421-425. https://doi.org/https://doi.org/10.1016/j.scijus.2016.06.007. 
(39) Kara, I.; Sarikavak, Y.; Lisesivdin, S. B.; Kasap, M. Evaluation of Morphological and Chemical Differences of Gunshot Residues in Different Ammunitions Using SEM/EDS Technique. Environ. Forensics 2016, 17 (1), 68-79. https://doi.org/10.1080/15275922.2015.1133729.

(40) Andrasko, J.; Maehly, A. C. Detection of Gunshot Residues on Hands by Scanning Electron Microscopy. J. Forensic Sci. 1977, 22 (2), 279-287.

(41) Basu, S. Formation of Gunshot Residues. J. Forensic Sci. 1982, 27 (1), 72-91. https://doi.org/10.1520/jfs11453j.

(42) Hannigan, T. J.; McDermott, S. D.; Greaney, C. M.; O’Shaughnessy, J.; O’Brien, C. M. Evaluation of Gunshot Residue (GSR) Evidence: Surveys of Prevalence of GSR on Clothing and Frequency of Residue Types. Forensic Sci. Int. 2015, 257, 177-181.

(43) Kara, I. The Relationship between Gunshot-Residue Particle Size and Boltzmann Distribution. Forensic Sci. Res. 2020, 6. https://doi.org/10.1080/20961790.2020.1713433.

(44) Menking-Hoggatt, K.; Martinez, C.; Vander Pyl, C.; Heller, E.; Pollock, E. “Chip”; Arroyo, L.; Trejos, T. Development of Tailor-Made Inorganic Gunshot Residue (IGSR) Microparticle Standards and Characterization with a Multi-Technique Approach. Talanta 2021. https://doi.org/10.1016/j.talanta.2020.121984.

(45) Dalby, O.; Butler, D.; Birkett, J. W. Analysis of Gunshot Residue and Associated Materials: A Review. J. Forensic Sci. 2010, 55 (4), 924-943. https://doi.org/10.1111/j.1556-4029.2010.01370.x.

(46) Abrego, Z.; Grijalba, N.; Unceta, N.; Maguregui, M.; Sanchez, A.; Fernandez-Isla, A.; Goicolea, M. A.; Barrio, R. J. A Novel Method for the Identification of Inorganic and Organic Gunshot Residue Particles of Lead-Free Ammunitions from the Hands of Shooters Using Scanning Laser Ablation-ICPMS and Raman Micro-Spectroscopy. Analyst 2014, 139 (23), 6232-6241. https://doi.org/10.1039/c4an01051e.

(47) Nonlead Ammunition in California http://www.wildlife.ca.gov/hunting/nonleadammunition.

(48) Mason, K.; Wuhrer, R. Detection and Characterization of Heavy-Metal-Free (HMF) Gunshot Residues Using CL, EDS and Raman Together with XRD. Mircoscopic Microanal. 2018, 24 (1), 1174-1175. https://doi.org/10.1017/S1431927618006359. (49) Oommen, Z.; Pierce, S. M. Lead-Free Primer Residues: A Qualitative Characterization of 
Winchester WinClean (TM), Remington/UMC LeadLess (TM), Federal BallistiClean (TM), and Speer Lawman CleanFire (TM) Handgun Ammunition. J. Forensic Sci. 2006, 51 (3), 509-519. https://doi.org/10.1111/j.1556-4029.2006.00107.x.

(50) Martiny, A.; Campos, A. P. C.; Sader, M. S.; Pinto, M. A. L. SEM/EDS Analysis and Characterization of Gunshot Residues from Brazilian Lead-Free Ammunition. Forensic Sci. Int. 2008, 177 (1), E9-E17. https://doi.org/10.1016/j.forsciint.2007.07.005.

(51) Gunaratnam, L.; Himberg, K. The Identification of Gunshot Residue Particles from LeadFree Sintox Ammunition. J. Forensic Sci. 1994, 39 (2), 532-536. https://doi.org/10.1520/jfs13626j.

(52) Charpentier, B.; Desrochers, C. Analysis of Primer Residue from Lead Free Ammunition by X-Ray Microfluorescence. J. Forensic Sci. 2000, 45 (2), 447-452. https://doi.org/10.1520/jfs14705j.

(53) Zeichner, A.; Levin, N. More on the Uniqueness of Gunshot Residue (GSR) Particles. $J$. Forensic Sci. TA - TT - 1997, 42 (6), 14255J. https://doi.org/10.1520/JFS14255J LK https://libwvu.on.worldcat.org/oclc/7355020110.

(54) Cook, M. Gunshot Residue Contamination of the Hands of Police Officers Following Start-of-Shift Handling of Their Firearm. Forensic Sci. Int. 2016, 269, 56-62. https://doi.org/10.1016/j.forsciint.2016.11.002.

(55) Blakey, L. S.; Sharples, G. P.; Chana, K.; Birkett, J. W. Fate and Behavior of Gunshot Residue-A Review. Journal of Forensic Sciences. Blackwell Publishing Inc. January 1, 2018, pp 9-19. https://doi.org/10.1111/1556-4029.13555.

(56) Doty, K. C.; Lednev, I. K. Raman Spectroscopy for Forensic Purposes: Recent Applications for Serology and Gunshot Residue Analysis. Trends Anal. Chem. 2018, 103, 215-222. https://doi.org/10.1016/j.trac.2017.12.003.

(57) Bueno, J.; Lednev, I. K. Advanced Statistical Analysis and Discrimination of Gunshot Residue Implementing Combined Raman and FT-IR Data Electronic Supplementary Information (ESI) Available. See DOI: 10.1039/C3ay40721g. Anal. Methods 2013, 5 (22), 6292-6296. https://doi.org/10.1039/c3ay40721g.

(58) Minzi?re, V. R.; Werner, D.; Schneider, D.; Manganelli, M.; Jung, B.; Weyermann, C.; Gassner, A. L. Combined Collection and Analysis of Inorganic and Organic Gunshot Residues. J. Forensic Sci. 2020, 65 (4), 1102-1113. https://doi.org/10.1111/1556- 
4029.14314.

(59) Bonnar, C.; Moule, E. C.; Lucas, N.; Seyfang, K. E.; Dunsmore, R. P.; Popelka-Filcoff, R. S.; Redman, K.; Paul Kirkbride, K. Tandem Detection of Organic and Inorganic Gunshot Residues Using LC-MS and SEM-EDS. Forensic Sci. Int. 2020, 314, 110389. https://doi.org/https://doi.org/10.1016/j.forsciint.2020.110389.

(60) Laza, D.; Nys, B.; Kinder, J. D.; Kirsch-De Mesmaeker, A.; Moucheron, C. Development of a Quantitative LC-MS/MS Method for the Analysis of Common Propellant Powder Stabilizers in Gunshot Residue. J. For. Sci. 2007, 52 (4), 842-850.

(61) McKenzie-Coe, A.; Bell, S.; Fernandez-Lima, F. Detection of Firearm Discharge Residue from Skin Swabs Using Trapped Ion Mobility Spectrometry Coupled to Mass Spectrometry. Anal. Methods 2018, 10 (35), 7. https://doi.org/10.1039/c8ay00658j.

(62) Vuki, M.; Shiu, K. K.; Galik, M.; O’Mahony, A. M.; Wang, J. Simultaneous Electrochemical Measurement of Metal and Organic Propellant Constituents of Gunshot Residues. Analyst 2012, 137 (14), 3265-3266.

(63) Werner, D.; Gassner, A. L.; Marti, J.; Christen, S.; Wyss, P.; Weyermann, C. Comparison of Three Collection Methods for the Sodium Rhodizonate Detection of Gunshot Residues on Hands. Sci. Justice 2020, 60 (1), 63-71. https://doi.org/10.1016/j.scijus.2019.09.004.

(64) Chohra, M.; Beladel, B.; Baba Ahmed, L.; Mouzai, M.; Akretche, D.; Zeghdaoui, A.; Mansouri, A.; Benamar, M. E. A. E. A. Study of Gunshot Residue by NAA and ESEM/EDX Using Several Kinds of Weapon and Ammunition. J. Radiat. Res. Appl. Sci. 2015, 8 (3), 404-410. https://doi.org/10.1016/j.jrras.2015.02.012.

(65) Koons, R. D.; Havekost, D. G.; Peters, C. A. Analysis of Gunshot Primer Residue Collection Swabs Using Flameless Atomic-Absorption Spectrophotometry and Inductively Coupled Plasma-Atomic Emission-Spectrometry-Effects of a Modified Extraction Procedure and Storage of Standards. J. Forensic Sci. 1989, 34 (1), 218-221. https://doi.org/10.1520/jfs12624j.

(66) Koons, R. D. Flameless Atomic Absorption Spectrophotometric Determination of Antimony and Barium in Gunshot Residue Collection Swabs: A Collaborative Study. Crime Lab. Dig. 1993, 20 (1), 19-23.

(67) B., Y.; A., O.-Y.; T., B.; N., S.; Z., K.; Yüksel, B.; Ozler-Yigiter, A.; Bora, T.; Sen, N.; Kayaalti, Z. GFAAS Determination of Antimony, Barium, and Lead Levels in Gunshot 
Residue Swabs: An Application in Forensic Chemistry LK -

Https://Libwvu.on.Worldcat.Org/Oclc/6873036042. At. Spectrosc. TA - TT - 2016, 37 (4), 164-169. https://doi.org/10.46770/as.2016.04.006.

(68) Costa, R. A.; dosSantos, N. A.; Correa, T. S. M.; Wyatt, N. L. P.; Chamoun, C. A.; Carneiro, M.; Romao, W. Detection of $\mathrm{Pb}, \mathrm{Ba}$, and $\mathrm{Sb}$ in Cadaveric Maggots and Pupae by ICP-MS*. J. Forensic Sci. 2020, 65 (6), 2188-2193. https://doi.org/10.1111/15564029.14526.

(69) Koons, R. D.; Havekost, D. G.; Peters, C. A. Determination of Barium in Gunshot Residue Collection Swabs Using Inductively Coupled Plasma-Atomic EmissionSpectrometry. J. Forensic Sci. 1988, 33 (1), 35-41.

(70) Vanini, G.; Souza, R. M.; Destefani, C. A.; Merlo, B. B.; Piorotti, T. M.; de Castro, E. V. R.; Carneiro, M.; Romao, W. Analysis of Gunshot Residues Produced by .38 Caliber Handguns Using Inductively Coupled Plasma-Optical Emission Spectroscopy (ICP OES). Microchem. J. 2014, 115, 106-112. https://doi.org/10.1016/j.microc.2014.03.003.

(71) Halim, M. I. A.; Safian, M. F.; Elias, E.; Shazali, S. S.; Abdul Halim, M. I.; Safian, M. F.; Elias, E.; Shazali, S. S. Identification of Gunshot Residue from Trace Element by Using ICP/OES Identifikasi Residu Tembakan Pistol Daripada Unsur Surih Menggunakan ICP/OES. In 2013 IEEE Symposium on Computers \& Informatics (ISCI); IEEE, 2013; pp 231-235. https://doi.org/10.1109/ISCI.2013.6612409.

(72) Aliste, M.; Arranz, S.; S?nchez-Ortega, A.; Sampedro, M. C.; Unceta, N.; G?mezCaballero, A.; Vallejo, A.; Goicolea, M. A.; Barrio, R. J. Particle Analysis for the Detection of Gunshot Residue (GSR) in Nasal Samples Using Scanning Laser Ablation and Inductively Coupled Plasma-Mass Spectrometry (SLA-ICPMS). J. Forensic Sci. 2020, 65 (4), 1094-1101. https://doi.org/10.1111/1556-4029.14278.

(73) Turillazzi, E.; Di Peri, G. P.; Nieddu, A.; Bello, S.; Monaci, F.; Neri, M.; Pomara, C.; Rabozzi, R.; Riezzo, I.; Fineschi, V. Analytical and Quantitative Concentration of Gunshot Residues ( $\mathrm{Pb}, \mathrm{Sb}, \mathrm{Ba})$ to Estimate Entrance Hole and Shooting-Distance Using Confocal Laser Microscopy and Inductively Coupled Plasma Atomic Emission Spectrometer Analysis: An Experimental Study. Forensic Sci. Int. 2013, 231 (1), 142149. https://doi.org/https://doi.org/10.1016/j.forsciint.2013.04.006.

(74) Diaz, E.; Souza Sarkis, J. E.; Viebig, S.; Saldiva, P. Measurement of Airborne Gunshot 
Particles in a Ballistics Laboratory by Sector Field Inductively Coupled Plasma Mass Spectrometry. Forensic Sci. Int. TA - TT - 2012, 214 (1-3), 44-47.

https://doi.org/10.1016/j.forsciint.2011.07.016 LK https://libwvu.on.worldcat.org/oclc/5902539928.

(75) Lagoo, L.; Schaeffer, L. S.; Szymanski, D. W.; Smith, R. W. Detection of Gunshot Residue in Blowfly Larvae and Decomposing Porcine Tissue Using Inductively Coupled Plasma Mass Spectrometry (ICPMS)*. J. Forensic Sci. 2010, 55 (3), 624-632. https://doi.org/10.1111/j.1556-4029.2010.01327.x.

(76) Dona-Fernandez, A.; de Andres-Gimeno, I.; Santiago-Toribio, P.; Valtuille-Fernandez, E.; Aller-Sanchez, F.; Heras-Gonzalez, A. Real-Time Detection of GSR Particles from Crime Scene: A Comparative Study of SEM/EDX and Portable LIBS System. Forensic Sci. Int. 2018, 292, 167-175. https://doi.org/10.1016/j.forsciint.2018.09.021.

(77) Senesi, G. S.; Harmon, R. S.; Hark, R. R. Field-Portable and Handheld Laser-Induced Breakdown Spectroscopy: Historical Review, Current Status and Future Prospects. Spectrochim. Acta Part B At. Spectrosc. 2021, 175, 106013. https://doi.org/https://doi.org/10.1016/j.sab.2020.106013.

(78) Rinke-Kneapler, C. N.; Sigman, M. E. Applications of Laser Spectroscopy in Forensic Science. In Laser Spectroscopy for Sensing: Fundamentals, Techniques and Applications; Baudelet, M., Ed.; Woodhead Publ Ltd: Cambridge, 2014; pp 461-495. https://doi.org/10.1533/9780857098733.3.461.

(79) Niewöhner, L.; Barth, M.; Neimke, D.; Latzel, S.; Stamouli, A.; Nys, B.; Gunaratnam, L.; Fries, K.; Uhlig, S.; Baldauf, H. Development, Design, and Realization of a Proficiency Test for the Forensic Determination of Shooting Distances - FDSD 2015. Forensic Chem. 2016, 1, 22-30. https://doi.org/10.1016/j.forc.2016.06.002.

(80) Gandy, L.; Najjar, K.; Terry, M.; Bridge, C. A Novel Protocol for the Combined Detection of Organic, Inorganic Gunshot Residue. Forensic Chem. 2018, 8, 1-10. https://doi.org/https://doi.org/10.1016/j.forc.2017.12.009.

(81) Lee, H.-C.; Meng, H.-H. The Identification of Two Unusual Types of Homemade Ammunition. J. Forensic Sci. 2012, 57, 1102-1107.

(82) Chang, K. H.; Jayaprakash, P. T.; Yew, C. H.; Abdullah, A. F. L. Gunshot Residue Analysis and It Evidential Values: A Review. Aust. J. Forensic Sci. 2013, 45 (1), 3-23. 
https://doi.org/10.1080/00450618.2012.691546.

(83) Alvarez, A.; Yanez, J. Screening of Gunshot Residue in Skin Using Attenuated Total Reflection Fourier Transform Infrared (ATR FT-IR) Hyperspectral Microscopy. Appl. Spectrosc. 2020, 74 (4), 400-407. https://doi.org/10.1177/0003702819892930.

(84) Stevens, B.; Bell, S.; Adams, K. Initial Evaluation of Inlet Thermal Desorption GC-MS Analysis for Organic Gunshot Residue Collected from the Hands of Known Shooters. Forensic Chem. 2016, 2, 55-62. https://doi.org/https://doi.org/10.1016/j.forc.2016.10.001.

(85) Tarifa, A.; Almirall, J. R. Fast Detection and Characterization of Organic and Inorganic Gunshot Residues on the Hands of Suspects by CMV-GC-MS and LIBS. Sci. Justice 2015, 55 (3), 168-175. https://doi.org/10.1016/j.scijus.2015.02.003.

(86) Zeichner, A.; Eldar, B.; Glattstein, B.; Koffman, A.; Tamiri, T.; Muller, D. Vacuum Collection of Gunpowder Residues from Clothing Worn by Shooting Suspects, and Their Analysis by GC/TEA, IMS, and GC/MS. J. Forensic Sci. 2003, 48 (5), 2002390. https://doi.org/10.1520/jfs2002390.

(87) Gallidabino, M.; Weyermann, C.; Romolo, F. S.; Taroni, F. Estimating the Time since Discharge of Spent Cartridges: A Logical Approach for Interpreting the Evidence. Sci. Justice 2013, 53 (1), 41-48. https://doi.org/10.1016/j.scijus.2011.12.004.

(88) Thomas, J. L.; Lincoln, D.; McCord, B. R. Separation and Detection of Smokeless Powder Additives by Ultra Performance Liquid Chromatography with Tandem Mass Spectrometry (UPLC/MS/MS). J. For. Sci. 2013, 58 (3), 609-615.

(89) Maitre, M.; Kirkbride, K. P.; Horder, M.; Roux, C.; Beavis, A. Thinking beyond the Lab: Organic Gunshot Residues in an Investigative Perspective. Aust. J. Forensic Sci. 2018, 50 (6), 659-665. https://doi.org/10.1080/00450618.2018.1457718.

(90) Castellanos, A.; Bell, S.; Fernandez-Lima, F. Characterization of Firearm Discharge Residues Recovered from Skin Swabs Using Sub-Micrometric Mass Spectrometry Imaging. Anal. Methods 2016, 8 (21), 4300-4305. https://doi.org/10.1039/c6ay00096g.

(91) Zuy, Y.; Sweck, S. O.; Dockery, C. R.; Potts, G. E. HPLC Detection of Organic Gunshot Residues Collected with Silicone Wristbands. Anal. Methods 2020, 12 (1), 85-90. https://doi.org/10.1039/c9ay02305d.

(92) Yeager, B.; Bustin, K.; Stewart, J.; Dross, R.; Bell, S. Evaluation and Validation of Ion Mobility Spectrometry for Presumptive Testing Targeting the Organic Constituents of 
Firearms Discharge Residue. Anal. Methods 2015, 7 (22), 9683-9691.

https://doi.org/10.1039/c5ay02417j.

(93) Harshey, A.; Das, T.; Srivastava, A. Analytical Contributions of Lanthanide Based MetalOrganic Frame Works as Luminescent Markers: Recent Trends in Gunshot Residue Analysis. Microchem. J. 2020, 154, 6. https://doi.org/10.1016/j.microc.2020.104597.

(94) Pluháček, T.; Švidrnoch, M.; Maier, V.; Havlíček, V.; Lemr, K. Laser Ablation Inductively Coupled Plasma Mass Spectrometry Imaging: A Personal Identification Based on a Gunshot Residue Analysis on Latent Fingerprints. Anal. Chim. Acta 2018, 1030, 2532. https://doi.org/https://doi.org/10.1016/j.aca.2018.05.074.

(95) Brozek-Mucha, Z. Distribution and Properties of Gunshot Residue Originating from a Luger 9 Mm Ammunition in the Vicinity of the Shooting Gun. Forensic Sci. Int. 2009, 183 (1-3), 33-44. https://doi.org/10.1016/j.forsciint.2008.10.010.

(96) Thomas, R. Practical Guide to ICP-MS : A Tutorial for Beginners. Third edit. CRC Press: Boca Raton, FL 2013.

(97) Olesik, J. W. Elemental Analysis Using ICP-OES and ICP/MS. Anal. Chem. 1991, 63 (1), 12A-21A. https://doi.org/10.1021/ac00001a001.

(98) Vanhaecke, F.; Degryse, P.; Wiley, I. Isotopic Analysis : Fundamentals and Applications Using ICP-MS. Wiley-VCH: Weinheim 2012.

(99) Khandpur, R. S. Handbook of Analytical Instruments. 2nd editio. McGraw-Hill Education LLC: New York, N.Y. 2006.

(100) Schijf, J.; Garvin, M. C. Validation and Application of a New Microwave-Digestion/ICPMS Method for the Analysis of Trace Metals in Tree Increment Cores. Geochem. J. 2018, 52 (4), 347-358. https://doi.org/10.2343/geochemj.2.0524.

(101) Chevallier, E.; Chekri, R.; Zinck, J.; Guerin, T.; Noel, L. Simultaneous Determination of 31 Elements in Foodstuffs by ICP-MS after Closed-Vessel Microwave Digestion: Method Validation Based on the Accuracy Profile. J. Food Compos. Anal. 2015, 41, 35-41. https://doi.org/10.1016/j.jfca.2014.12.024.

(102) Astolfi, M. L.; Marconi, E.; Protano, C.; Vitali, M.; Schiavi, E.; Mastromarino, P.; Canepari, S. Optimization and Validation of a Fast Digestion Method for the Determination of Major and Trace Elements in Breast Milk by ICP-MS. Anal. Chim. Acta 2018, 1040, 49-62. https://doi.org/10.1016/j.aca.2018.07.037. 
(103) Koons, R. D. Analysis of Gunshot Primer Residue Collection Swabs by Inductively Coupled Plasma-Mass Spectrometry. J. Forensic Sci. 1998, 43 (4), 748-754. https://doi.org/10.1520/jfs14301j.

(104) Abrego, Z.; Ugarte, A.; Unceta, N.; Fernández-Isla, A.; MA, G.; RJ, B. Unambiguous Characterization of Gunshot Residue Particles Using Scanning Laser Ablation and Inductively Coupled Plasma-Mass Spectrometry. Anal. Chem. TA - TT - 2012, 84 (5), 2402-2409. https://doi.org/10.1021/ac203155r LK https://libwvu.on.worldcat.org/oclc/779544517.

(105) Ferreira, I. M. de S.; Braz, B. F.; da Silva, L.; Luna, A. S.; Santelli, R. E. Gunshot Residue and Gunshot Residue-like Material Analysis Using Laser Ablation Inductively Coupled Plasma Mass Spectrometry Imaging. Spectrochim. Acta Part B At. Spectrosc. TA - TT 2021, 177. https://doi.org/10.1016/j.sab.2021.106087 LK https://libwvu.on.worldcat.org/oclc/8893745971.

(106) Hendriks, L.; Gundlach-Graham, A.; Günther, D. Performance of Sp-ICP-TOFMS with Signal Distributions Fitted to a Compound Poisson Model. J. Anal. At. Spectrom. 2019, 34 (9), 1900-1909. https://doi.org/10.1039/c9ja00186g.

(107) Goldstein, J.; Newbury, D. E.; Michael, J. R.; Ritchie, N. W. M.; Scott, J. H. J.; Joy, D. C. Scanning Electron Microscopy and X-Ray Microanalysis. Fourth edi. Springer: New York, NY 2018. https://doi.org/10.1007/978-1-4939-6676-9.

(108) Ul-Hamid, A. A Beginners' Guide to Scanning Electron Microscopy. Springer: Cham, Switzerland 2018. https://doi.org/10.1007/978-3-319-98482-7.

(109) Rakovan, J. A Word to the Wise: Energy Dispersive Spectrometry (EDS). Rocks Miner. 2004, 79 (3), 194-195.

(110) Speaker, P. J. Project FORESIGHT Annual Report, 2018-2019; West Virginia University, 2020.

(111) Musazzi, S.; Perini, U. Laser-Induced Breakdown Spectroscopy : Theory and Applications. Springer: Berlin, Heidelberg 2014. https://doi.org/10.1007/978-3-64245085-3.

(112) Noll, R. Laser-Induced Breakdown Spectroscopy: Fundamentals and Applications; Springer-Verlag Berlin Heidelberg: Heidelberg ;, 2012. https://doi.org/10.1007/978-3-64220668-9. 
(113) Rakovský, J.; Čermák, P.; Musset, O.; Veis, P. A Review of the Development of Portable Laser Induced Breakdown Spectroscopy and Its Applications. At. Spectrosc. 2014, 101 (C), 269-287.

(114) Yang, P.; Zhou, R.; Zhang, W.; Yi, R.; Tang, S.; Guo, L.; Hao, Z.; Li, X.; Lu, Y.; Zeng, X. High-Sensitivity Determination of Cadmium and Lead in Rice Using Laser-Induced Breakdown Spectroscopy. Food Chem. 2019, 272, 323-328.

https://doi.org/https://doi.org/10.1016/j.foodchem.2018.07.214.

(115) Motto-Ros, V.; Syvilay, D.; Bassel, L.; Negre, E.; Trichard, F.; Pelascini, F.; El Haddad, J.; Harhira, A.; Moncayo, S.; Picard, J.; Devismes, D.; Bousquet, B. Critical Aspects of Data Analysis for Quantification in Laser-Induced Breakdown Spectroscopy. Spectrochim. Acta Part B-Atomic Spectrosc. 2018, 140, 54-64. https://doi.org/10.1016/j.sab.2017.12.004.

(116) Rammelkamp, K.; Schröder, S.; Ortenzi, G.; Pisello, A.; Stephan, K.; Baqué, M.; Hübers, H.-W.; Forni, O.; Sohl, F.; Thomsen, L.; Unnithan, V. Field Investigation of Volcanic Deposits on Vulcano, Italy Using a Handheld Laser-Induced Breakdown Spectroscopy Instrument. Spectrochim. Acta Part B At. Spectrosc. 2021, 177, 106067. https://doi.org/https://doi.org/10.1016/j.sab.2021.106067.

(117) López-López, M.; Delgado, J. J.; García-Ruiz, C. Ammunition Identification by Means of the Organic Analysis of Gunshot Residues Using Raman Spectroscopy. Anal. Chem. 2012, 84 (8), 3581-3585. https://doi.org/10.1021/ac203237w.

(118) Mason, K.; Wuhrer, R. Phenom Desktop SEM for Gunshot Residue and Cathodoluminescence Imaging and Analysis. Mircoscopic Microanal. 2017, 23 (1), 2. https://doi.org/10.1017/S1431927617006031.

(119) O’Mahony, A. M.; Wang, J. Electrochemical Detection of Gunshot Residue for Forensic Analysis: A Review. Electroanalysis 2013, 25 (6), 1341-1358. https://doi.org/10.1002/elan.201300054.

(120) Taudte, R. V.; Beavis, A.; Blanes, L.; Cole, N.; Doble, P.; Roux, C. Detection of Gunshot Residues Using Mass Spectrometry. BioMed. Res. Int. 2014, 2014 (3), 1-16. https://doi.org/10.1155/2014/965403.

(121) Lucas, N.; Cook, M.; Kirkbride, K. P.; Kobus, H. Gunshot Residue Background on Police Officers: Considerations for Secondary Transfer in GSR Evidence Evaluation. Forensic 
Sci. Int. 2019, 297 (2019), 293-301. https://doi.org/10.1016/j.forsciint.2019.02.017.

(122) Lucas, N.; Cook, M.; Wallace, J.; Kirkbride, K. P.; Kobus, H. Quantifying Gunshot Residues in Cases of Suicide: Implications for Evaluation of Suicides and Criminal Shootings. Forensic Sci. Int. 2016, 266, 289-298. https://doi.org/https://doi.org/10.1016/j.forsciint.2016.06.006.

(123) French, J.; Morgan, R. An Experimental Investigation of the Indirect Transfer and Deposition of Gunshot Residue: Further Studies Carried out with SEM-EDX Analysis. Forensic Sci. Int. 2015, 247, 14-17. https://doi.org/10.1016/j.forsciint.2014.10.023.

(124) Moran, J. W.; Bell, S. Skin Permeation of Organic Gunshot Residue: Implications for Sampling and Analysis. Anal. Chem. 2014, 86 (12), 6071-6079. https://doi.org/10.1021/ac501227e.

(125) Berk, R. E.; Rochowicz, S. A.; Wong, M.; Kopina, M. A. Gunshot Residue in Chicago Police Vehicles and Facilities: An Empirical Study. J. Forensic Sci. 2007, 52 (4), 838841. https://doi.org/10.1111/j.1556-4029.2007.00457.x.

(126) Gassner, A.-L.; Ribeiro, C.; Kobylinska, J.; Zeichner, A.; Weyermann, C. Organic Gunshot Residue: Observations about Sampling and Transfer Mechanisms. Forensic Sci. Int. 2016, 266, 369-378.

(127) Szynkowska, M. I.; Parczewski, A.; Szajdak, K.; Rogowski, J. Examination of Gunshot Residues Transfer Using ToF-SIMS. Surf. Interface Anal. 2013, 45 (1), 596-600. https://doi.org/10.1002/sia.5142.

(128) Greely, D.; Weber, E. Transfer and Distribution of Gunshot Residue through Glass Windows. J. Forensic Sci. TA - TT - 2017, 62 (4), 869-873. https://doi.org/10.1111/1556-4029.13331 LK https://libwvu.on.worldcat.org/oclc/7081465646.

(129) Brozek-Mucha, Z.; Brożek-Mucha, Z. On the Prevalence of Gunshot Residue in Selected Populations - An Empirical Study Performed with SEM-EDX Analysis. Forensic Sci. Int. TA - TT - 2014, 237 (2014), 46-52. https://doi.org/10.1016/j.forsciint.2014.01.020 LK https://libwvu.on.worldcat.org/oclc/5900381250.

(130) Gauriot, R.; Gunaratnam, L.; Moroni, R.; Reinikainen, T.; Corander, J. Statistical Challenges in the Quantification of Gunshot Residue Evidence. J. Forensic Sci. 2013, 58 (5), 1149-1155. https://doi.org/10.1111/1556-4029.12179. 
(131) Andrasko, J.; Stahling, S. Time since Discharge of Spent Cartridges. J. Forensic Sci. 1999, 44 (3), 487-495.

(132) Andrasko, J.; Stahling, S. Time since Discharge of Pistols and Revolvers. J. Forensic Sci. 2003, 48 (2), 307-311.

(133) Andrasko, J.; Norberg, T.; Stahling, S. Time since Discharge of Shotguns. J. Forensic Sci. 1998, 43 (5), 1005-1015. https://doi.org/10.1520/jfs14349j.

(134) Andrasko, J.; Stahling, S. Time since Discharge of Rifles. J. Forensic Sci. 2000, 45 (6), $1250-1255$.

(135) Gassner, A. L.; Weyermann, C. Prevalence of Organic Gunshot Residues in Police Vehicles. Sci. Justice 2020, 60 (2), 136-144. https://doi.org/10.1016/j.scijus.2019.09.009.

(136) Brozek-Mucha, Z. Variation of the Chemical Contents and Morphology of Gunshot Residue in the Surroundings of the Shooting Pistol as a Potential Contribution to a Shooting Incidence Reconstruction. Forensic Sci. Int. 2011, 210 (1-3), 31-41. https://doi.org/10.1016/j.forsciint.2011.01.031.

(137) Arndt, J.; Bell, S.; Crookshanks, L.; Lovejoy, M.; Oleska, C.; Tulley, T.; Wolfe, D. Preliminary Evaluation of the Persistence of Organic Gunshot Residue. Forensic Sci. Int. 2012, 222 (1-3), 137-145. https://doi.org/10.1016/j.forsciint.2012.05.011.

(138) Berk, R. E. Automated SEM/EDS Analysis of Airbag Residue I: Particle Identification. $J$. Forensic Sci. 2009, 54 (1), 60-68. https://doi.org/10.1111/j.1556-4029.2008.00918.x.

(139) Tucker, W.; Lucas, N.; Seyfang, K. E.; Kirkbride, K. P.; Popelka-Filcoff, R. S. Gunshot Residue and Brakepads: Compositional and Morphological Considerations for Forensic Casework. Forensic Sci. Int. 2017, 270, 76-82.

(140) Lafleche, D. J. N.; Briere, S. J. J.; Faragher, N. F.; Hearns, N. G. R. Gunshot Residue and Airbags: Part I. Assessing the Risk of Deployed Automotive Airbags to Produce Particles Similar to Gunshot Residue. Can. Soc. Forensic Sci. J. 2018, 51 (2), 48-57. https://doi.org/10.1080/00085030.2018.1463202.

(141) Berk, R. E. Automated SEM/EDS Analysis of Airbag Residue. II: Airbag Residue as a Source of Percussion Primer Residue Particles. J. Forensic Sci. 2009, 54 (1), 69-76. https://doi.org/10.1111/j.1556-4029.2008.00919.x.

(142) Grima, M.; Butler, M.; Hanson, R.; Mohameden, A. Firework Displays as Sources of Particles Similar to Gunshot Residue. Sci. Justice 2012, 52 (1), 49-57. 
https://doi.org/10.1016/j.scijus.2011.04.005.

(143) Anders, D. H.; Miller, S. A.; Graziano, C. R.; Castellano, J.; Conte, J. Technical Note: Presence of Gunshot Residue in and around a Police Station. Int. J. Legal Med. 2020, 134 (6), 2195-2198. https://doi.org/10.1007/s00414-020-02357-2.

(144) Ali, L.; Brown, K.; Castellano, H.; Wetzel, S. J. A Study of the Presence of Gunshot Residue in Pittsburgh Police Stations Using SEM/EDS and LCMS/MS. J. Forensic Sci. 2016, 61 (4), 928-938. https://doi.org/10.1111/1556-4029.13077.

(145) Charles, S.; Geusens, N. A Study of the Potential Risk of Gunshot Residue Transfer from Special Units of the Police to Arrested Suspects. Forensic Sci. Int. 2012, 216 (1), 78-81. https://doi.org/https://doi.org/10.1016/j.forsciint.2011.08.022.

(146) Bell, S.; Seitzinger, L. From Binary Presumptive Assays to Probabilistic Assessments: Differentiation of Shooters From Non-Shooters Using IMS, OGSR, Neural Networks, and Likelihood Ratios. Forensic Sci. Int. 2016, 263, 176-185.

(147) Biedermann, A.; Bozza, S.; Taroni, F. Probabilistic Evidential Assessment of Gunshot Residue Particle Evidence (Part II): Bayesian Parameter Estimation for Experimental Count Data. Forensic Sci. Int. 2011, 206 (1-3), 103-110. https://doi.org/10.1016/j.forsciint.2010.07.009.

(148) Biedermann, A.; Bozza, S.; Taroni, F. Probabilistic Evidential Assessment of Gunshot Residue Particle Evidence (Part I): Likelihood Ratio Calculation and Case Pre-Assessment Using Bayesian Networks. Forensic Sci. Int. 2009, 191 (1-3), 24-35. https://doi.org/10.1016/j.forsciint.2009.06.004.

(149) El Haddad, J.; Canioni, L.; Bousquet, B. Good Practices in LIBS Analysis: Review and Advices. Spectrochim. Acta Part B-Atomic Spectrosc. 2014, 101, 171-182. https://doi.org/10.1016/j.sab.2014.08.039.

(150) Gallidabino, M. D.; Barron, L. P.; Weyermann, C.; Romolo, F. S. Quantitative ProfileProfile Relationship (QPPR) Modelling: A Novel Machine Learning Approach to Predict and Associate Chemical Characteristics of Unspent Ammunition from Gunshot Residue (GSR). Analyst 2019, 144 (4), 1128-1139. https://doi.org/10.1039/C8AN01841C.

(151) Kumar, R.; Sharma, V. Chemometrics in Forensic Science. Trends Anal. Chem. 2018, 105, 191-201. https://doi.org/10.1016/j.trac.2018.05.010.

(152) Yañez, J.; Paz Farías, M.; Zúñiga, V.; Soto, C.; Contreras, D.; Pereira, E.; Mansilla, H. D.; 
Saavedra, R.; Castillo, R.; Sáez, P. Differentiation of Two Main Ammunition Brands in Chile by Regularized Discriminant Analysis (RDA) of Metals in Gunshot Residues.

Microchem. J. 2012, 101, 43-48.

https://doi.org/https://doi.org/10.1016/j.microc.2011.10.003.

(153) Dai, S. Y.; Sang, H.; Lee, K. M.; Herrman, T. J. Cost of Speed: A Practical Approach to Evaluate a Screening Method from a Bayesian Perspective. Chemom. Intell. Lab. Syst. 2016, 156, 273-279. https://doi.org/10.1016/j.chemolab.2016.05.019.

(154) Kleinbaum, D. G.; Klein, M. Logistic Regression : A Self-Learning Text. 3rd ed. Springer: New York 2010. https://doi.org/10.1007/978-1-4419-1742-3.

(155) Bali, R.; Sarkar, D.; Lantz, B.; Lesmeister, C. R : Unleash Machine Learning Techniques : Find out How to Build Smarter Machine Learning Systems with R : Follow This Three Module Course to Become a More Fluent Machine Learning Practitioner : A Course in Three Modules. Packt Publishing: Birmingham, UK 2016.

(156) Marini, F. Artificial Neural Networks in Foodstuff Analyses: Trends and Perspectives A Review. Anal. Chim. Acta 2009, 635 (2), 121-131. https://doi.org/10.1016/j.aca.2009.01.009.

(157) Wasserman, P. D. Neural Computing : Theory and Practice; Van Nostrand Reinhold: New York, 1989.

(158) Ellison, S. L. R. Uncertainties in Qualitative Testing and Analysis. Accred. Qual. Assur. 2000, 5, 346-348.

(159) Ellison, S. L. R.; Fearn, T. Characterising the Performance of Qualitative Analytical Methods: Statistics and Terminology. Trends Anal. Chem. 2005, 24 (6), 468-476.

(160) Mandel Micha, M.; Azulay, O. I.; Zidon, Y.; Tsach, T.; Cohen, Y. Classification Improvements in Automated Gunshot Residue (GSR) Scans. J. Forensic Sci. 63 (4), 1269-1274.

(161) Damary, N. K.; MandelY, M.; Levin, N.; Izraeli, E. Calculation of Likelihood Ratios for Gunshot Residue Evidence-Statistical Aspects. Law, Probab. Risk 2016, 15 (2), 107-125. https://doi.org/10.1093/lpr/mgw001.

(162) Rijnders, M. R.; Stamouli, A.; Bolck, A. Comparison of GSR Composition Occurring at Different Locations Around the Firing Position. J. Forensic Sci. TA - TT - 2010, 55 (3), 616-623. https://doi.org/10.1111/j.1556-4029.2009.01292.x LK - 
https://libwvu.on.worldcat.org/oclc/5155841637.

(163) Bolck, A.; Stamouli, A. Likelihood Ratios for Categorical Evidence; Comparison of LR Models Applied to Gunshot Residue Data. Law Probab. Risk 2017, 16 (3), 19.

(164) Taroni, F.; Biedermann, A.; Garbolino, P.; Aitken, C. G. G. A General Approach to Bayesian Networks for the Interpretation of Evidence. Forensic Sci. Int. 2004, 139 (1), 516. https://doi.org/10.1016/j.forsciint.2003.08.004.

(165) Jensen, F. V.; Nielsen, T. D. Bayesian Networks and Decision Graphs. Statistics for Engineering and Information Science; 2007.

(167) Giannelli, Paul C., "Gunshot Residue Tests" (1991). Faculty Publications. 335. 


\section{Section 2}
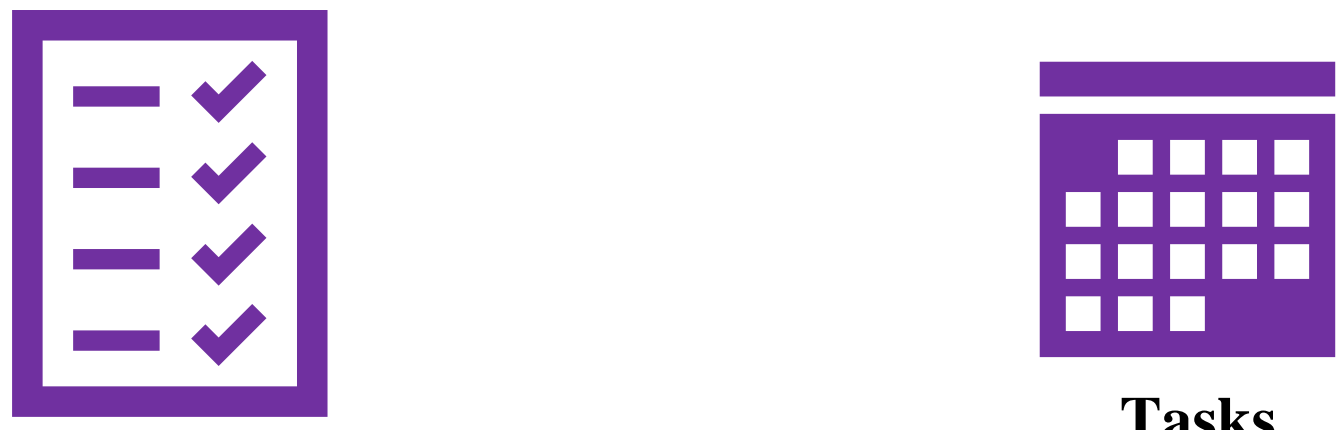

Objectives

Tasks

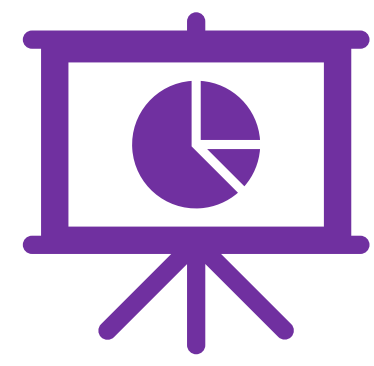

Deliverables

Overall Goals, Objectives, Tasks, and Deliverables

General Chapter Outline 


\section{Project Design and Objectives}

\subsection{Overall Project Goals}

The overall goal of this study was to enhance the reliability of GSR evidence by speeding up detection, increasing the body of knowledge on chemical composition of modern cartridge residues, and validating statistical methods for the quantitative assessment of the evidential value of GSR. This goal was achieved through two major objectives: 1) characterization of the composition of IGSR from modern ammunition, 2) validation of the reliability of Laser Induced Breakdown Spectroscopy (LIBS) and Scanning Electron Microscopy- Electron Dispersive X-ray Spectroscopy (SEM-EDS).

The objectives were accomplished by the statistical analysis of a population that includes hand residues collected at 200 individuals who have fired characterized ammunitions originating from leaded and lead-free cartridges and 200 non-shooter backgrounds originating from low-risk and high-risk individuals. Upon completion of this research, samples were collected from 975 individuals from the different population types, resulting in over 2,900 samples/ GSR stubs analyzed by LIBS. Moreover, a novel approach for the creation of tailor-made microparticle pGSR standards provided a unique opportunity to characterize modern ammunition. The project consisted of eight specific tasks listed in the next section under their respective objectives (Figure $1)$.

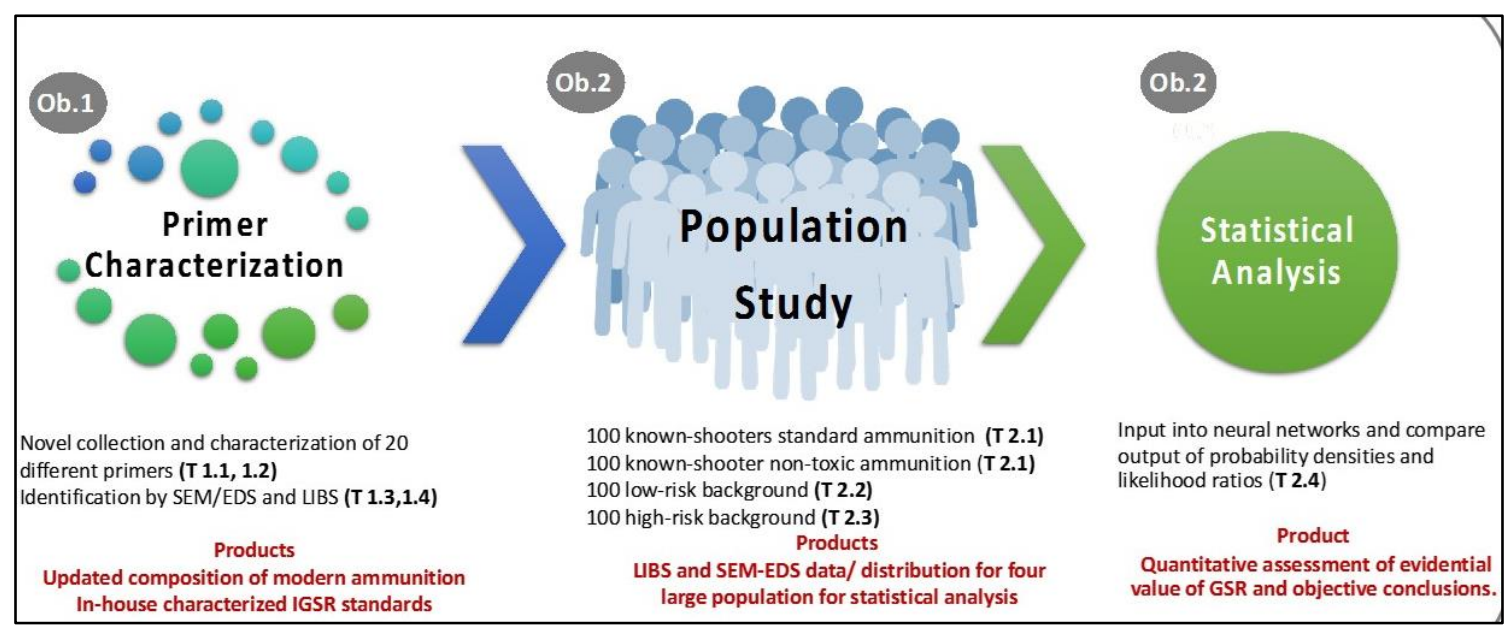

Figure 1: Flowchart of project implementation and method. Objective $=\mathrm{Ob}$. Task $=\mathrm{T}$ 


\subsection{Objectives and Respective Tasks}

\section{Objective 1 and respective tasks}

Objective 1. Qualitative and quantitative characterization of multiple types of standard and leadfree primers using a novel collection approach and the combination of three different analytical techniques for characterization purposes: Inductively Coupled Plasma- Mass Spectrometry (ICPMS), SEM-EDS and LIBS.

Task 1.1. Collect standard primers (fifteen) and lead-free primers (ten) by directly recovering the primer particles in a polypropylene container and then suspending them in acetone. Casings were fired without the bullet or the propellant in order to identify the GSR residues coming directly from the primer. Each primer was collected in three replicates for a total of 75 samples (45 standard and 30 lead-free primers).

Task 1.2. Characterize the primer samples qualitatively and quantitatively using ICP-MS. This spectrochemical method was only proposed as a confirmation test for the primer's bulk concentration and not for the application to casework.

Task 1.3 Deposit a known volume of the characterized in-house primer suspensions onto a SEM specimen carbon stub and analyze by SEM-EDS to monitor morphology and to determine if the elemental composition agrees with the anticipated chemical profiles. The IGSR particle count per volume were estimated for a small portion of the in-house standards.

Task 1.4. Characterize the primers by LIBS using the GSR carbon stub commonly employed in GSR collection. The LIBS results were compared to ICP-MS and SEM-EDS to confirm the elemental composition of the sample. The controlled conditions of primer deposits on the SEM-EDS stubs allows the evaluation of the spatial distributions of the LIBS chemical mapping and estimation the number of IGSR particles detected.

\section{Objective 2 and respective tasks}

Objective 2. Validation of the reliability of LIBS and SEM-EDS by the statistical analysis of a population that included hand residues from known shooters and non-shooters using the characterized leaded and lead-free ammunitions, along with low and high-risk background populations. 
Task 2.1. Collect and analyze by LIBS sample sets consisting of four stubs each the control-known shooters hands after firing leaded and lead-free ammunition with the characterized and uncharacterized primers. When collecting characterized ammunition, the type of bullet, the cartridge case, and the gunpowder remained constant. A total metal jacket bullet was used to ensure no lead contamination from the core of the bullet. A random selection of 100 stubs (50 leaded and 50 lead-free) were analyzed by SEM-EDS. The target number of samples in this set, was initially established for 200 sets (100 leaded and 100 lead-free). However, the goal was exceeded for a total of 520 samples analyzed by LIBS (200 leaded, 100 lead-free, and 220 mixed shooter sets). Also, SEM-EDS analysis was completed on 52 leaded samples and 56 lead-free samples.

Task 2.2 Collect and analyze by LIBS 100 sets consisting of two stubs each from the lowrisk background population. The exception was 50 individuals consisting of only one stub each collected from the dominant hand. A random selection of 52 stubs were analyzed by SEM-EDS.

Task 2.3 Collect and analyze by LIBS 100 sets consisting of two stubs each from the highrisk background population. A total of 105 high-risk sets were analyzed by LIBS. A selection of 50 stubs are in progress to be analyzed by SEM-EDS, starting first with any positive stubs and then selecting the remaining stub randomly.

Task 2.4 Conduct data preprocessing and statistical analysis. Identify the relevant elements which contain the most information about the absence or presence of GSR. The data was then be processed by different types of machine learning algorithms to create a predictive model based on the respective classification (i.e., low-risk background, high-risk background, shooter with standard ammunition, or shooter with lead-free ammunition). The different machine learning outputs are used to generate probability density distributions and likelihood ratios.

\subsection{Deliverables}

This project was funded by NIJ under award \# 2018-R2-CX-0009, and this dissertation corresponds to one of the deliverables expected upon completion of the project grant, which will end June $30^{\text {th }}$, 2021. In addition to the financial, progress, and products required by the funding agency, other deliverables of this research include the dissemination of data collected within the 
forensic examiners and stakeholders. All data sets and methods for data processing created during this study will be made available to interested stakeholders and archived by the NIJ. As part of the dissemination strategy, the PI published two scientific publications in peer-review journals, shared research results at 19 different scientific meetings in the form of posters and oral presentations, and an additional publication about the population study is in preparation.

\subsection{Chapter Structure}

This dissertation describes the accomplishments of each of the goals into 4 main chapters. Section 3 (Chapter 1) describes a full validation and ruggedness test of an ICP-MS digestion method for the characterization of tailor-made IGSR standards. This was necessary to establish that the developed method was fit for the purpose of detecting the elements present in tailor-made standards and had the ability to determine their elemental composition. The evaluation of the analytical performance of the ICP-MS for detection and quantitation of GSR particles served as an essential basis to this study. A portion of this chapter was published as supplemental material in Talanta. ${ }^{1}$

Section 4 (Chapter 2) describes a novel approach for the creation of a microparticle standard for the detection of gunshot residues. In this study, tailor-made standards that simulate different inorganic gunshot residues from leaded and non-toxic ammunition were created and characterized. Inductively Coupled Plasma Mass Spectrometry (ICP-MS), Scanning Electron Microscopy Energy Dispersive X-Ray Spectroscopy (SEM-EDS), and Laser-Induced Breakdown Spectroscopy (LIBS) methods were used to characterize the elemental composition of the microparticles, to evaluate the morphology, and their stability. The ASTM standard practice for SEM-EDS analysis was followed as the gold standard for the examination of GSR, while ICP-MS and LIBS methods were developed as alternative tools for the analysis and characterization of the standards.

The current dearth of GSR standard materials limits the study of gunshot residues in fields such as forensic and environmental sciences, where the research of transfer, fate, and persistence of residues derived from a firearm is of interest. One advantage of the proposed standard materials is its flexibility to be used in suspension or dry form, as it allows for its use for a multitude of techniques and matrices. The results show that the proposed approach can provide a leap of knowledge by offering a laboratory standard material that can be used for quality control, 
interlaboratory testing, and development and validation of conventional and emerging analytical methodologies for GSR detection. The research presented in this chapter was published in Talanta as the main article with the supplemental material mentioned previously. ${ }^{1}$ Additional standards and replicates were characterized since the time of the publication of the article. Therefore, this chapter expands to the reported findings in section 4 chapter 2.

Section 5 (Chapter 3) presents the development of novel LIBS method for detection of gunshot residue. The proposed LIBS method is capable of documenting the micro-spatial distribution of IGSR components on substrates dabbed from an individual's hands. The 3D spectrochemical data includes simultaneous information about the analyte characteristic wavelengths, their respective intensity, and $\mathrm{x}-\mathrm{y}$ location coordinates within the sample.

The study compared the efficiency of single spot analysis to raster line ablation patterns. Superior performance was obtained with the micro-spot method, while offering the possibility of performing GSR particle analysis instead of micro-bulk sampling. The simultaneous detection of characteristic elements on a given ablation time and from a single micro-region increases the confidence that the trace originated from GSR rather than other non-GSR sources. In addition, this approach allows the collection of 25 individual spectra per stub adding more information to the analysis. Finally, this chapter reports the use of machine learning algorithms for the interpretation of GSR data and its validation with a large set of authentic specimens. Logistic Regression, Naïve Bayes, and Neural Network provided effective predictive methods, with classification accuracy better than $93.7 \%$ for a validation set of 326 authentic samples collected from shooters and background populations. This chapter was published in the Journal of Chemometrics. ${ }^{3}$

Section 6: Chapter 4 presents the results of the large population study performed on leaded and lead-free shooters, along with the low and high-risk background samples. We examined the distribution of GSR elements of interest in the background population and determined the base thresholds for different elements. The GSR profiles were analyzed by LIBS for a set of nearly one thousand individuals, from almost 3,000 samples, generating over 80,0000 spectral data files. A subset of 200 items were also characterized SEM-EDS on the same sample, after LIBS analysis. To the best of our knowledge, this is the largest population study conducted on GSR from individual's hands. A study of this size also allowed for the use of predictive machine learning methods that provide probabilities outputs. The data was then used to determine likelihood ratios to aid in a probabilistic interpretation of GSR evidence. Section 6/ Chapter 4 will be part of an 
article in preparation, where we will also include the results from electrochemical analysis in our results and interpretation.

\subsection{References}

(1) Menking-Hoggatt, K.; Martinez, C.; Vander Pyl, C.; Heller, E.; Pollock, E. “Chip”; Arroyo, L.; Trejos, T. Development of Tailor-Made Inorganic Gunshot Residue (IGSR) Microparticle Standards and Characterization with a Multi-Technique Approach. Talanta 2021. https://doi.org/10.1016/j.talanta.2020.121984.

(2) Trejos, T.; Vander Pyl, C.; Menking-Hoggatt, K.; Alvarado, A. L.; Arroyo, L. E. Fast Identification of Inorganic and Organic Gunshot Residues by LIBS and Electrochemical Methods . Forensic Chem. 2018, 8, 146-156.

https://doi.org/doi.org/10.1016/j.forc.2018.02.006.

(3) Menking-Hoggatt, K.; Arroyo, L.; Curran, J.; Trejos, T. Novel LIBS Method for MicroSpatial Chemical Analysis of Inorganic Gunshot Residue. J. Chemom. 2019, e3208, 13. 


\section{Section 3}

\section{Validation, digestion, and detection of 34 elements}
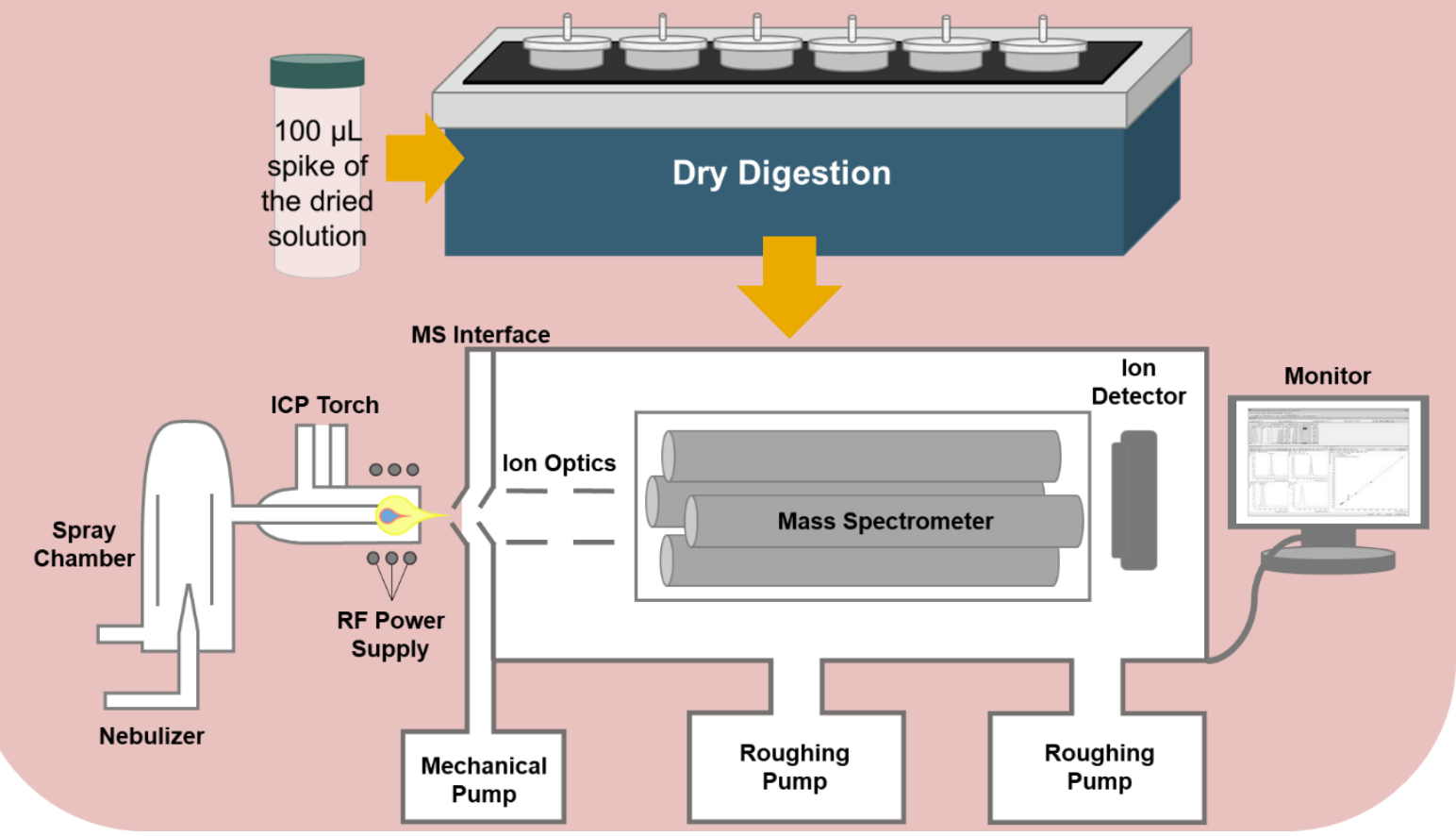

\section{Chapter 1}

Development and Validation of an ICP-MS Digestion Method for the Bulk Characterization of Tailor-Made IGSR Standards 


\section{Chapter 1: Development and Validation of an ICP-MS Digestion Method for the Bulk Characterization of Tailor-Made IGSR Standards}

\subsection{Overview}

The field of GSR in the forensic community currently only uses SEM-EDS for the analysis of evidence, therefore there is only one type of standard material available and it is designed specifically for SEM-EDS analysis and is not consumable. Through a collaboration with a publicly funded crime laboratory (Sacramento, CA), we developed a collection method for IGSR that originated only from the ammunition primer and mimics authentic GSR. Before fully characterizing the tailor-made IGSR standards, we developed a method for the bulk characterization of the standards by ICP-MS. Due to the novelty of this standard material, an exhaustive extraction and quantitative detection method needed development, optimization and validation to determine that it was fit for our purpose of bulk characterization of the elemental concentration of GSR components.

To accomplish this, we carried out ruggedness testing to establish the effect of small changes in the method conditions on the quantitative abilities of the method. We conducted the ruggedness test using the Plackett-Burman design and determine the factors that needed to be tightly controlled and monitored during the procedure. After determining this, we completed a full validation of the developed method. Figures of merits were evaluated: selectivity, working range, limits of detection, limits of quantitation, trueness, and precision. From these results, we determined if the values were within acceptable ranges for the reproducible and accurate monitoring of the bulk elemental concentration of the tailor-made IGSR standards over time as well as established quality control protocols for the next stage of the project. These results were included as part of the supplemental material for the research article published in Talanta. ${ }^{1}$

\subsection{Introduction}

One of this study's objectives was to characterize the elemental composition of modern ammunition of tailored-made microparticle GSR standards. To achieve a complete characterization, a multiple technique approach was designed using ICP-MS for the bulk quantitative composition of the standards, SEM-EDS for elemental composition and particle shape, and LIBS as an additional elemental analysis tool. Therefore, the ICP-MS method was 
created to gain a deep knowledge of the GSR standards' chemical composition that can be further used as a "ground" truth for the additional application of the GSR standard materials. Still, ICPMS is not intended to be used as a technique for analyzing authentic GSR samples or for casework due to its destructive nature.

Before beginning the full characterization study of the tailor-made IGSR standards, a hot block acid digestion followed by ICP-MS was optimized as an exhaustive extraction and detection method to characterize and quantify the bulk elemental composition of GSR-related elements. ICPMS digestion method was developed and validated for 34 elements of interest $\left({ }^{7} \mathrm{Li},{ }^{9} \mathrm{Be},{ }^{11} \mathrm{~B},{ }^{23} \mathrm{Na}\right.$, ${ }^{24} \mathrm{Mg},{ }^{27} \mathrm{Al},{ }^{28} \mathrm{Si},{ }^{39} \mathrm{~K},{ }^{44} \mathrm{Ca},{ }^{47} \mathrm{Ti},{ }^{51} \mathrm{~V},{ }^{52} \mathrm{Cr},{ }^{55} \mathrm{Mn},{ }^{57} \mathrm{Fe},{ }^{60} \mathrm{Ni},{ }^{63} \mathrm{Cu},{ }^{66} \mathrm{Zn},{ }^{71} \mathrm{Ga},{ }^{85} \mathrm{Rb},{ }^{88} \mathrm{Sr},{ }^{90} \mathrm{Zr},{ }^{93} \mathrm{Nb}$, ${ }^{97} \mathrm{Mo},{ }^{118} \mathrm{Sn},{ }^{121} \mathrm{Sb},{ }^{133} \mathrm{Cs},{ }^{137} \mathrm{Ba},{ }^{157} \mathrm{Gd},{ }^{178} \mathrm{Hf},{ }^{181} \mathrm{Ta},{ }^{182} \mathrm{~W},{ }^{195} \mathrm{Pt},{ }^{208} \mathrm{~Pb},{ }^{209} \mathrm{Bi}$ ), and 3 internal standards $\left({ }^{45} \mathrm{Sc}\right.$ for low $\mathrm{m} / \mathrm{z}$ analytes, ${ }^{89} \mathrm{Y}$ for intermediate $\mathrm{m} / \mathrm{z}$ analytes, ${ }^{113} \mathrm{In}$ for high $\mathrm{m} / \mathrm{z}$ analytes). The elements were chosen based on an exhaustive research literature review and patents about the composition of modern ammunition, ${ }^{2-8}$ along with the list of elements provided in the ASTM 1588-20 standard for GSR analysis. ${ }^{9}$ The method was validated according to recommendations from the EPA 6020B standard and the Eurachem guidelines. ${ }^{10,11}$ These documents were used as references for the quality control and validation design, along with defining the acceptable range of recoveries.

Validation of new method is the first step in any method development. Any new method must determine if the procedure and parameters are "fit for the purpose" of the experimentation, and the equipment being used is working correctly, within the specifications provided by the manufacturer, and properly calibrated. ${ }^{11}$ This can be performed on the single laboratory scale or be conducted by multiple laboratories performing interlaboratory testing of new, emerging methods. Either way, the goal is the same, to ensure the method is valid and statistical analysis of the method uses generally accepted terminology. In the case of the ICP-MS digestions method, we needed to ensure that the results would accurately determine the bulk concentration of the tailormade IGSR standards over the course of a year long stability study. We examined the interday and intraday variability to determine if the results would be reproducible over time and have acceptable recovery of the analytes of interest, so we had an accurate understanding of the true concentration of the tailor-made IGSR standards.

Our validation design began with a ruggedness test following the protocol described in the ASTM 1169-18 standard for the Plackett-Burman design. ${ }^{12}$ The experimental design was used to 
develop the most efficient and reliable method by assessing the controlled variables before proceeding to the full-validation stage. Ruggedness, or robustness, testing is not always performed during validation, but we chose to start with this experiment to ensure that any factors that greatly affected our recovery of analytes were tightly controlled and monitored during the validation study to ensure the validation was performed with the proper controls in place and the more influential factors were controlled.

After ruggedness testing, the figures of merit assessed were selectivity, limit of detection (LOD), limit of quantitation (LOQ), working range, trueness, and precision; all of which are essential for establishing if the developed method is fit for purpose. Selectivity was the first step and involved researching the monoisotopic and polyatomic interferences of the $\mathrm{m} / \mathrm{z}$ analytes of interest and choosing the $\mathrm{m} / \mathrm{z}$ with fewest interferences, along with testing the type of gas used in the collision cell, which helps to reduce the creation polyatomic compounds. Since the method is quantitative, the next thing we determined was the working range of the method and the subsequent LOD and LOQ of the method. This was necessary to determine the linearity of the working range, along with the lowest level at which we could be confident that an element was present (LOD) and when concentration could be confidently determine (LOQ). In quantitative techniques, the LOD is lower than the LOQ.

Once those figures of merit were established, then we could evaluate the trueness and precision of the developed method, and therefore the quality of the results with the appropriate uncertainty attached. Trueness can be compared to the how close a mean of an infinite number of results is to a reference value, but since this is an impractical approach, we use bias and recovery to assess this concept and compare it with a reference material with a known concentration. ${ }^{11}$ Precision examines the replication of the method, taking into account all the different operational conditions that might arise when the method is applied. We assessed this by calculation the Relative Standard Deviation (RSD\%) within the same day (intraday variability) and between different days (interday variability). The purpose of the developed ICP-MS was to monitor the bulk concentrations over time, and the validated method needed to confirm that it was fit for purpose by having low LOQ, was accurate to the true concentration in the solution, and had acceptable precision over time. 


\subsection{Materials and Methods}

\subsubsection{Ruggedness Testing for Digestion Efficiency}

The ruggedness experiment was conducted to assess six factors at two levels each (Table 1), and their effect on the daily performance of the method was measured and statistically examined the factors that greatly affect the outcome of the results. Once the critical factors are identified, the method can be designed to control for these variables during a typical analysis and to improve efficiency. Statistical analysis of the Plackett-Burman experimental design was completed using the statistical software JMP Pro 14.0.0 (SAS Institute Inc., NC) using the concentrations reported by ICP-MS as the response. The results of the ruggedness testing provided a robust and efficient method for the analysis of the IGSR microparticle samples collected that could be carried forward through a full validation.

Table 1: Factors and levels chosen for the Plackett-Burman experimental design.

\begin{tabular}{lcc}
\hline Factor & Low Level & High Level \\
\hline Digestion Temperature $\left({ }^{\circ} \mathbf{C}\right)$ & 80 & 100 \\
\hline Digestion Time $(\mathbf{h})$ & 1 & 2 \\
\hline Time between preparation and digestion (days) & 0 & 2 \\
\hline Time between digestion and analysis (days) & 0 & 2 \\
\hline Acid Concentration $(\mathbf{v} / \mathbf{v} \%)$ & 5 & 10 \\
\hline Spike Amount $(\boldsymbol{\mu g} / \mathbf{L})$ & 5 & 50 \\
\hline
\end{tabular}

\subsubsection{Validation Figures of Merit}

Standard solutions were prepared using ICP-MS quality standards ( $\mathrm{Li}, \mathrm{Be}, \mathrm{B}, \mathrm{Na}, \mathrm{Mg}, \mathrm{Al}$, $\mathrm{Si}, \mathrm{K}, \mathrm{Ca}, \mathrm{Ti}, \mathrm{V}, \mathrm{Cr}, \mathrm{Mn}, \mathrm{Fe}, \mathrm{Ni}, \mathrm{Cu}, \mathrm{Zn}, \mathrm{Ga}, \mathrm{Rb}, \mathrm{Sr}, \mathrm{Zr}$, Nb, Mo, Sn, Sb, Cs, Ba, Gd, Hf, Ta, W, $\mathrm{Pt}, \mathrm{Pb}, \mathrm{Bi}$ ) at 25 and $50 \mu \mathrm{g} / \mathrm{L}$, acid nitric optima grade (XXX) and deionized water (XXX). Scandium, Ytriem and Indium were used as internal standards (Alfa Aesar, Ward Hill, MA; Accu standard, New Haven, CT; Inorganic ventures, Christiansburg, VA; RICCA, Arlington, TX; Ultra scientific, Kingstown, RI; VHG Labs, Manchester, NH).

The selectivity was initially evaluated by monitoring isobaric and polyatomic interferences present for the analytes of interest. During analysis, selectivity was assessed daily using an interference mix that contained all the analytes of interest, internal standards, and 2\% (v/v) Optima grade nitric acid matrix solution. Daily performance of the instrument was monitored through a daily performance report and a tune report. The report provided the general background counts 
and sensitivity for low, medium, and high elements, along with the doubly charged ratio for cobalt and the oxide ratio of cerium oxide to cerium. In order to monitor run performance, two quality control (QC) samples at 25 and $50 \mu \mathrm{g} / \mathrm{L}$ were analyzed every 20 samples and contained all the elements of interest in the same $2 \%(\mathrm{v} / \mathrm{v})$ nitric acid solution as the calibration curve. These samples also served as continuing calibration verification samples (CCV). A typical run consisted of 40-70 samples, in addition to QC samples and the calibration curve.

The limits of detection (LOD) and quantitation (LOQ) were evaluated using the reagent blank samples containing only the blank acetone sample and internal standards. The standard deviation of the blank was calculated using eleven replicate samples using the formula shown in Equation 1. The calculation for LOD and LOQ reflected both the number of method reagent blanks $(n)$ and instrument blanks analyzed by ICP-MS $\left(n_{b}\right)$. The method blanks consisted of eleven

$$
s^{\prime}{ }_{0}=s_{0} \sqrt{\frac{1}{n}+\frac{1}{n_{b}}}
$$

Equation 1: Formula for LOD calculation with the standard deviation $\left(s^{\prime}{ }_{0}\right)$ from the experimental standard deviation $\left(s_{0}\right)$, the number of replicate observations subjected to the entire procedure $(n)$, and the number of observations used to calculate the blank correction $\left(n_{b}\right)$ during analysis.

replicate samples subjected to the entire digestion process, while the instrument blank was a $2 \%$ (v/v) nitric acid and internal standard solution that was not digested.

A 14-point calibration curve $(0-300 \mu \mathrm{g} / \mathrm{L})$ was selected to determine the working range of the method. The working range was tested to account for small amounts $(0-5 \mu \mathrm{g} / \mathrm{L})$ and for higher concentrations of the analyte in a sample $(100-300 \mu \mathrm{g} / \mathrm{L})$ as per the Eurachem guidelines. ${ }^{11}$ The calibration curve was analyzed on five different days to determine the acceptable working range of the method by assessing normality, homoscedasticity, and linearity over time.

Method trueness was evaluated through bias using relative spike recovery and percent recovery. Nine samples were spiked with the analytes of interest at two concentration levels (25 and $50 \mu \mathrm{g} / \mathrm{L}$ ), along with the internal standard. The replicate samples were digested, diluted, and analyzed by ICP-MS on the same day for a total of 18 samples, not including QC and calibration curve samples. Percent recovery and relative recovery were calculated using Equation 2 and 3, respectively, for the $25 \mu \mathrm{g} / \mathrm{L}$ and $50 \mu \mathrm{g} / \mathrm{L}$ samples. The results were examined to determine if the ranges were in acceptable tolerance according to the EPA 6020 standard. ${ }^{10}$ 
The interday and intraday relative standard deviations (RSD) of the percent recovery were used to evaluate the precision of the developed method. A spiked amount of either 25 or $50 \mu \mathrm{g} / \mathrm{L}$ for the 34 different elements was added to each sample on four different days using 6-9 replicate per day. The RSD of the different analytes was calculated using the mean and standard deviation of the analyte concentrations. The results were assessed to determine if they were in acceptable range according to the EPA 6020 standard. ${ }^{10}$

$$
R(\%)=\frac{\bar{x}}{x_{\text {ref }}} \times 100
$$

$$
b(\%)=\frac{\bar{x}-x_{\mathrm{ref}}}{x_{\mathrm{ref}}} \times 100
$$

Equation 2: Formula for relative percent recovery using the average of the replicate samples subjected to the method $(\bar{x})$ and the target value spiked into the samples $\left(x_{\text {ref }}\right)$.
Equation 3: Formula for relative percent bias using the average of the replicate samples subjected to the method $(\bar{x})$ and the target value spiked into the samples $\left(x_{\text {ref }}\right)$.

\subsection{Results and Discussion}

\subsubsection{Ruggedness Testing of ICP-MS Digestion Methods}

A ruggedness test allows the identification of conditions that are critical to the overall method performance and the establishment of system suitability criteria. The implementation of the ruggedness test included aspects such as the selection of factors and levels to test, choosing an experimental design, interpreting the results, and finally, the application of the findings for the validated method.

Plackett-Burman experimental design (PB) was selected to determine the experimental parameters that must be closely controlled during routine analysis to ensure accurate and reproducible results. The $\mathrm{PB}$ experimental design is a statistical tool that estimates the main effects of different factors being tested. The larger the primary effect value is, the more significant the impact of the factor on the results of the experiment (in this study we monitored the percent recovery of analytes). Once the main effects with the highest impact on recovery have been identified, then a method and procedure can be designed to minimize variability of the results by tightly controlling and monitoring these factors.

In this study six main factors were evaluated at two levels: 1$)$ digestion temperature (80C or 100C), 2) digestion time (1 or 2 hours), 3) time between sample preparation and digestion (same 
day or 2 days after), 4) time between sample digestion and analysis (same day or 2 days after), 5) concentration of acid used for digestion ( $5 \%$ or 105), and 6) sample concentration (5 or $50 \mathrm{ug} / \mathrm{L}$ ). The response being monitored was percent recovery of isotopes of elements associated with inorganic gunshot residues (Table 2). The average response of all monitored elements was used as the standardized response factor for further calculations. To evaluate the main effects of the design, standardized effect calculations were performed according to the ASTM standard for conducting ruggedness testing (Table 2$)^{12}$

With an absolute standardized effect of 6.04, the acid concentration was determined to have the greatest impact on percent recovery of target gunshot residue elements. Additionally, time between preparation and digestion, and time between digestion and analysis also have a large effect on percent recovery with standardized effect values of 3.95 and 3.20, respectively. As a result, these determined main effects should be controlled when performing further analysis to ensure adequate recovery of all elements monitored. The last three factors of digestion time, digestion temperature, and spike concentration are less impactful and can be varied without percent recovery suffering.

A Plackett-Burman design assumes that main effects are, in general, heavily confounded with two-factor interactions. In order for the main effects to be meaningful, these interactions must be assumed to be negligible. To test the adequacy of the test, we evaluated if those assumptions were met in our data set by using correlation plots and alias matrices. The correlation plot and alias matrix showed that effects were only highly correlated to themselves and not correlated to other effects within the design, therefore, satisfying the assumption of orthogonal main effects (Figure 1 and Table 3). When effects are highly correlated, it becomes difficult to determine which specific effects are responsible for impacting the response. Furthermore, Plackett-Burman assumes that interactions between effects are negligible. This was also evaluated and validated using the correlation plots, proving that interaction between effects are negligible. In this graph, effects highly correlated will appear in red shade while low correlation will appear as blue shade with lowest correlations presented as darker blue. With all assumptions satisfied, the Plackett-Burman model was determined to be a valid design of experiment for ruggedness testing of the developed ICP/MS method for the analysis of the tailor-made IGSR microparticle standards. 
Table 2: Standardized effects calculation table for Plackett-Burman ruggedness test

\begin{tabular}{|c|c|c|c|c|c|c|c|}
\hline Pattern & $\begin{array}{l}\text { Digestion } \\
\text { Temperature } \\
\left({ }^{\circ} \mathrm{C}\right)\end{array}$ & $\begin{array}{l}\text { Digestion } \\
\text { Time (hrs) }\end{array}$ & $\begin{array}{l}\text { Time } \\
\text { between } \\
\text { preparation } \\
\text { and digestion } \\
\text { (days) }\end{array}$ & $\begin{array}{l}\text { Time } \\
\text { between } \\
\text { digestion and } \\
\text { analysis } \\
\text { (days) }\end{array}$ & $\begin{array}{l}\text { Acid } \\
\text { Concentratio } \\
\text { n }(\mathbf{v} / \mathbf{v} \%)\end{array}$ & $\begin{array}{l}\text { Spike } \\
\text { Amount } \\
(\mu \mathrm{g} / \mathrm{L})\end{array}$ & $\begin{array}{l}\text { Average } \\
\text { Percent } \\
\text { Recovery (\%) }\end{array}$ \\
\hline--+--+ & 80 & 1 & 2 & 0 & 5 & 50 & 102.0 \\
\hline+---+- & 100 & 1 & 0 & 0 & 10 & 5 & 96.8 \\
\hline-+--+- & 80 & 2 & 0 & 0 & 10 & 5 & 104.0 \\
\hline---+-- & 80 & 1 & 0 & 2 & 5 & 5 & 100.7 \\
\hline+--+-+ & 100 & 1 & 0 & 2 & 5 & 50 & 102.1 \\
\hline+++--- & 100 & 2 & 2 & 0 & 5 & 5 & 95.2 \\
\hline-+++-- & 80 & 2 & 2 & 2 & 5 & 5 & 124.3 \\
\hline++---+ & 100 & 2 & 0 & 0 & 5 & 50 & 99.5 \\
\hline++++++ & 100 & 2 & 2 & 2 & 10 & 50 & 99.2 \\
\hline+++--- & 100 & 2 & 2 & 0 & 5 & 5 & 119.7 \\
\hline ++---+ & 100 & 2 & 0 & 0 & 5 & 50 & 98.0 \\
\hline+---+ & 100 & 1 & 0 & 0 & 10 & 5 & 95.2 \\
\hline+-+++- & 100 & 1 & 2 & 2 & 10 & 5 & 104.2 \\
\hline-+--+- & 80 & 2 & 0 & 0 & 10 & 5 & 89.8 \\
\hline+---+- & 100 & 1 & 0 & 0 & 10 & 5 & 83.5 \\
\hline--+-++ & 80 & 1 & 2 & 0 & 10 & 50 & 97.8 \\
\hline-+-+++ & 80 & 2 & 0 & 2 & 10 & 50 & 97.7 \\
\hline-+-+++ & 80 & 2 & 0 & 2 & 10 & 50 & 96.7 \\
\hline---+-- & 80 & 1 & 0 & 2 & 5 & 5 & 103.1 \\
\hline--+--+ & 80 & 1 & 2 & 0 & 5 & 50 & 101.7 \\
\hline++++++ & 100 & 2 & 2 & 2 & 10 & 50 & 100.5 \\
\hline ++---+ & 100 & 2 & 0 & 0 & 5 & 50 & 99.9 \\
\hline-+-+++ & 80 & 2 & 0 & 2 & 10 & 50 & 97.5 \\
\hline-+-+++ & 80 & 2 & 0 & 2 & 10 & 50 & 96.7 \\
\hline+--+-+ & 100 & 1 & 0 & 2 & 5 & 50 & 100.9 \\
\hline--+--+ & 80 & 1 & 2 & 0 & 5 & 50 & 104.8 \\
\hline+-+++- & 100 & 1 & 2 & 2 & 10 & 5 & 87.0 \\
\hline+-+++- & 100 & 1 & 2 & 2 & 10 & 5 & 107.2 \\
\hline+--+-+ & 100 & 1 & 0 & 2 & 5 & 50 & 101.3 \\
\hline ++---+ & 100 & 2 & 0 & 0 & 5 & 50 & 101.5 \\
\hline--+-++ & 80 & 1 & 2 & 0 & 10 & 50 & 100.3 \\
\hline++++++ & 100 & 2 & 2 & 2 & 10 & 50 & 101.1 \\
\hline+++--- & 100 & 2 & 2 & 0 & 5 & 5 & 101.0 \\
\hline--+--+ & 80 & 1 & 2 & 0 & 5 & 50 & 105.9 \\
\hline-+++-- & 80 & 2 & 2 & 2 & 5 & 5 & 99.0 \\
\hline+--+-+ & 100 & 1 & 0 & 2 & 5 & 50 & 101.8 \\
\hline++++++ & 100 & 2 & 2 & 2 & 10 & 50 & 100.7 \\
\hline-+++-- & 80 & 2 & 2 & 2 & 5 & 5 & 99.0 \\
\hline-+++-- & 80 & 2 & 2 & 2 & 5 & 5 & 115.8 \\
\hline ---+-- & 80 & 1 & 0 & 2 & 5 & 5 & 93.8 \\
\hline+++--- & 100 & 2 & 2 & 0 & 5 & 5 & 88.4 \\
\hline-+--+- & 80 & 2 & 0 & 0 & 10 & 5 & 91.4 \\
\hline+-+++- & 100 & 1 & 2 & 2 & 10 & 5 & 94.3 \\
\hline +---+- & 100 & 1 & 0 & 0 & 10 & 5 & 94.1 \\
\hline-+--+- & 80 & 2 & 0 & 0 & 10 & 5 & 91.4 \\
\hline--+-++ & 80 & 1 & 2 & 0 & 10 & 50 & 99.8 \\
\hline---+-- & 80 & 1 & 0 & 2 & 5 & 5 & 112.0 \\
\hline \multirow[t]{2}{*}{--+-++} & 80 & 1 & 2 & 0 & 10 & 50 & 98.9 \\
\hline & & & & & & $\begin{array}{c}\text { Average } \\
\text { Response }\end{array}$ & 99.9 \\
\hline Average + & 98.96 & 100.34 & 101.84 & 101.52 & 96.91 & 100.23 & \\
\hline Average - & 100.51 & 99.51 & 97.89 & 98.32 & 102.94 & 99.62 & \\
\hline Main Effect & -1.55 & $\mathbf{0 . 8 3}$ & 3.95 & 3.20 & -6.04 & 0.62 & \\
\hline $\begin{array}{c}\text { Standardized } \\
\text { Effect }\end{array}$ & -1.55 & 0.83 & 3.95 & 3.20 & -6.04 & 0.62 & \\
\hline
\end{tabular}


Acid concentration, time between preparation and digestion, and time between digestion and analysis had the greatest impact on the recovery of target elements resulting in the largest standardized effect for the majority of the elements (Figure 2). As a result, 10\% nitric acid concentration was chosen to ensure the complete digestion of the IGSR microparticles into solution since all elements had recoveries between $90-110 \%$ at this level. Time between preparation and digestion, and time between digestion and analysis was difficult to control due to limited digestion space on the heating block system used in this study (only 10 samples in triplicate, plus controls could be processed at a time). However, samples were prepared at most one day before digestion and were run no more than $12 \mathrm{~h}$ after digestion was completed.

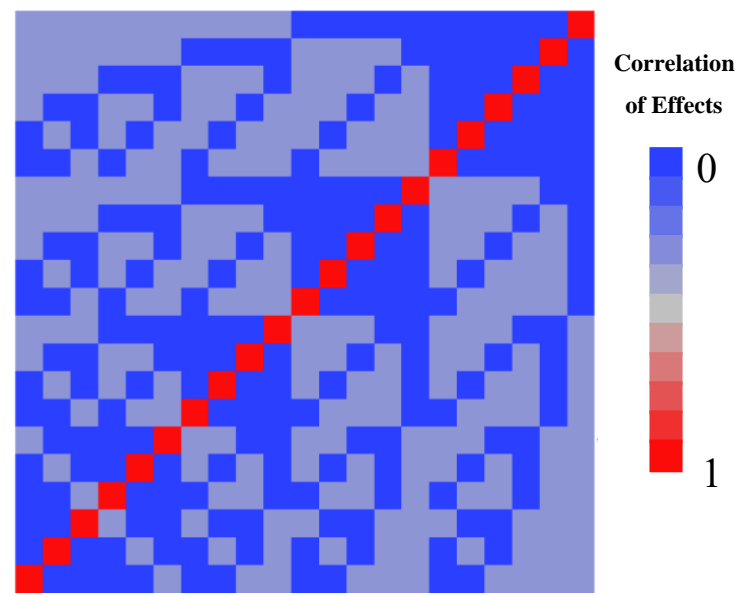

Figure 1: Correlation plot of all main effects tested for Plackett-Burman ruggedness testing. The plot visually demonstrated the correlation between each factor at two different levels with a cross-wise comparison of the different factors at the different levels. Each square is a comparison point, and the plot shown here shows low correlation between different factors. The diagonal red square down the center are the point when the same factor is compared with itself, therefore a high correlation is to be expected. 


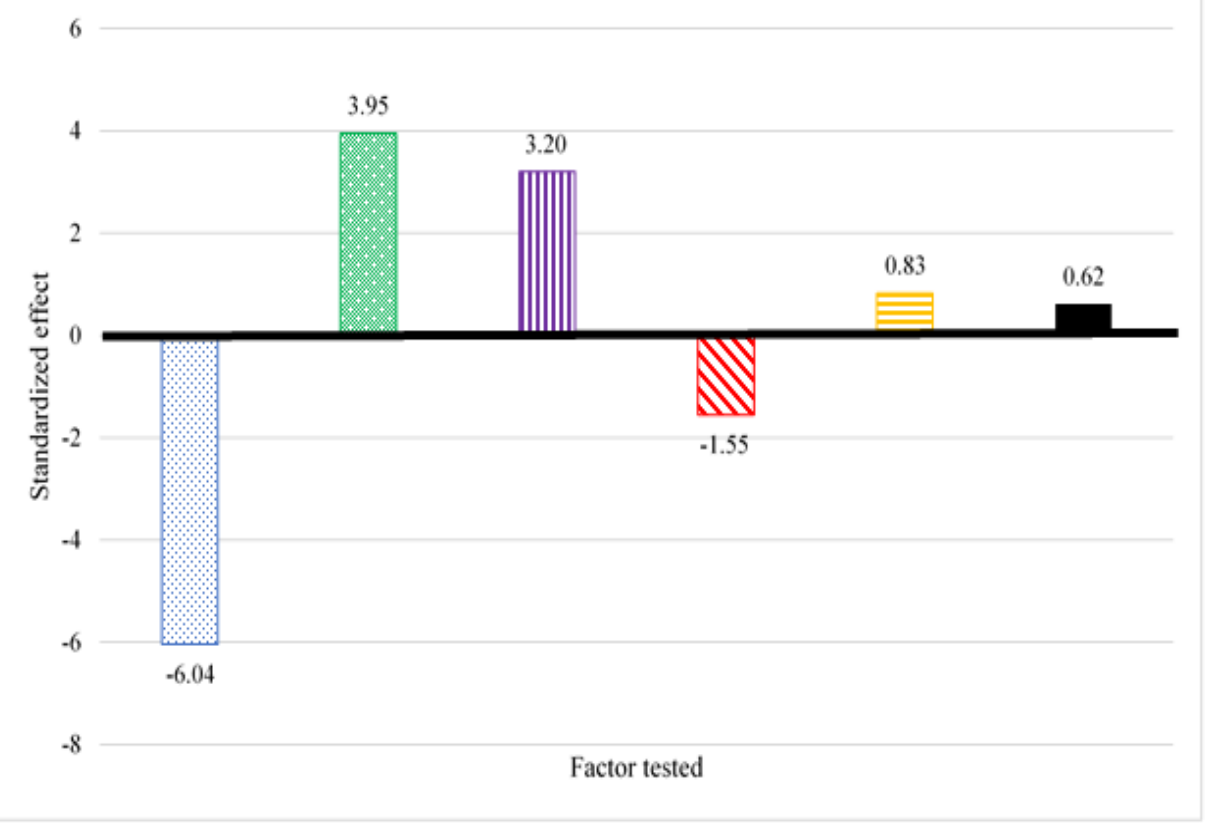

\section{Acid Concentration $\quad \mathbb{N}$ Digestion Temperature \\ Time between preparation and digestion}

血 Time between digestion and analysis $\quad \square$ Spike Amount

Figure 2: Average standardized effects chart of Plackett-Burman ruggedness testing. 
Table 3: Alias matrix of Plackett-Burman ruggedness test

\begin{tabular}{|c|c|c|c|c|c|c|}
\hline Effect & $\begin{array}{l}\text { Digestion Temperature } \\
\left({ }^{\circ} \mathrm{C}\right)^{*} \text { Digestion Time }(\mathrm{h})\end{array}$ & $\begin{array}{l}\text { Digestion Temperature } \\
\left({ }^{\circ} \mathrm{C}\right)^{*} \text { Time between } \\
\text { preparation and digestion } \\
\text { (days) }\end{array}$ & $\begin{array}{l}\text { Digestion Temperature } \\
\left({ }^{\circ} \mathrm{C}\right) * \text { Time between digestion } \\
\text { and analysis (days) }\end{array}$ & $\begin{array}{l}\text { Digestion Temperature } \\
\left({ }^{\circ} \mathbf{C}\right)^{*} \text { Acid Concentration } \\
(\mathrm{v} / \mathrm{v} \%)\end{array}$ & $\begin{array}{l}\text { Digestion Temperature } \\
\left({ }^{\circ} \mathrm{C}\right)^{*} \text { Spike Amount }(\mu \mathrm{g} / \mathrm{L})\end{array}$ & \\
\hline Intercept & 0 & 0 & 0 & 0 & 0 & \\
\hline Digestion Temperature $\left({ }^{\circ} \mathrm{C}\right)$ & 0 & 0 & 0 & 0 & 0 & \\
\hline Digestion Time (h) & 0 & -0.33 & 0.33 & 0.33 & -0.33 & \\
\hline Time between preparation and digestion (days) & -0.33 & 0 & -0.33 & -0.33 & 0.33 & \\
\hline Time between digestion and analysis (days) & 0.33 & -0.33 & 0 & -0.33 & -0.33 & \\
\hline Acid Concentration (v/v\%) & 0.33 & -0.33 & -0.33 & 0 & 0.33 & \\
\hline Spike Amount $(\mu \mathrm{g} / \mathrm{L})$ & -0.33 & -0.33 & -0.33 & 0.33 & 0 & \\
\hline Effect & $\begin{array}{l}\text { Time between preparation and } \\
\text { digestion (days)*Time between } \\
\text { digestion and analysis (days) }\end{array}$ & $\begin{array}{l}\text { Time between preparation and } \\
\text { digestion (days)*Acid } \\
\text { Concentration }(\mathbf{v} / \mathbf{v} \%)\end{array}$ & $\begin{array}{l}\text { Time between preparation and } \\
\text { digestion (days) }{ }^{*} \text { Spike Amount } \\
(\mu \mathrm{g} / \mathrm{L})\end{array}$ & $\begin{array}{l}\text { Time between digestion and } \\
\text { analysis (days)*Acid } \\
\text { Concentration }(\mathrm{v} / \mathrm{v} \%)\end{array}$ & $\begin{array}{l}\text { Time between digestion and } \\
\text { analysis (days)*Spike Amount } \\
(\mu \mathrm{g} / \mathrm{L})\end{array}$ & $\begin{array}{l}\text { Acid Concentration } \\
(\mathrm{v} / \mathrm{v} \%)^{*} \text { Spike Amount }(\mu \mathrm{g} / \mathrm{L})\end{array}$ \\
\hline Intercept & 0 & 0 & 0 & 0 & 0 & 0 \\
\hline Digestion Temperature $\left({ }^{\circ} \mathrm{C}\right)$ & -0.33 & -0.33 & 0.33 & -0.33 & -0.33 & 0.33 \\
\hline Digestion Time (h) & -0.33 & 0.33 & 0.33 & -0.33 & -0.33 & -0.33 \\
\hline Time between preparation and digestion (days) & 0 & 0 & 0 & -0.33 & 0.33 & -0.33 \\
\hline Time between digestion and analysis (days) & 0 & -0.33 & 0.33 & 0 & 0 & -0.33 \\
\hline Acid Concentration (v/v\%) & -0.33 & 0 & -0.33 & 0 & -0.33 & 0 \\
\hline Spike Amount $(\mu \mathrm{g} / \mathrm{L})$ & 0.33 & -0.33 & 0 & -0.33 & 0 & 0 \\
\hline
\end{tabular}


The rest of the factors affected the recoveries to a lesser extent, and their levels were chosen to simplify sample preparation while still maintaining the highest percent recovery for a majority of the elements. Concentrations of 25 and $50 \mu \mathrm{g} / \mathrm{L}$ were chosen for the spiked samples prepared and analyzed during each sample digestion. The lowest time $(1 \mathrm{~h})$ and temperature $\left(80^{\circ} \mathrm{C}\right)$ were chosen to ensure all the elements had satisfactory recovery $(90-110 \%)$ with the added benefit of reducing the sample preparation time and avoiding loss of volatiles at that digestion temperature.

\subsubsection{Figures of Merit}

After completing the ruggedness testing, a validation study was designed with the PB results incorporated into the ICP-MS digestion method procedure. The calculated figures of merit included selectivity, limit of detection (LOD), limit of quantitation (LOQ), trueness, and precision (Table 4).

Selectivity was established by researching and monitoring expected monoisotopic and polyatomic interferences for the different elements of interest. The chosen mass to charge $(\mathrm{m} / \mathrm{z})$ for specific elements were those with the least interferences. Polyatomic interferences from oxides and doubly charges species were monitored daily by measuring the oxide ratio using cobalt (75/59 $\mathrm{m} / \mathrm{z}$ ) and the doubly charged ratio using cerium $(70 / 140 \mathrm{~m} / \mathrm{z})$. Both ratios were less than $3 \%$ on the days the experiments were completed. The polyatomic interferences can be greatly reduced if helium gas is introduced into the collision chamber, so under helium mode the cobalt ratio was reduced to less than $1 \%$ during analysis.

The purpose of the developed digestion method was to determine the concentration of trace amounts of inorganic components present in modern IGSR by analyzing an external calibration with internal standard. All elements possessed a linear response with an $\mathrm{R}^{2}$ value of at least 0.999 except for silicon, calcium, iron, zinc, and cesium which had a value of 0.996 or higher (Table 4). The calibration working range was determined to be from 0 to $300 \mu \mathrm{g} / \mathrm{L}$ based on the high $\mathrm{R}^{2}$ values obtained from the linear line of fit for the replicate plots of the calibration points. Also, all the residuals were randomly distributed when compared to the predicted values, with less than $10 \%$ RSD. The residuals' variance did not increase with concentration, confirming that the data was normally distributed, linear, and possess homogeneous variance (homoscedasticity) across the data points. 
The validated measurement range allowed calculation of the method limits of detection (LOD) and quantitation (LOQ) for the 34 analytes of interest. The method LOD (Equation 1) refers to the lowest value at which an analyte of interest can be determined as present or absent in a qualitative manner. The method LOQ refers to the lowest value at which the analyte concentration can be obtained in a quantitative manner. ${ }^{11}$ Table 4 lists the method LOD and LOQ determined and the calculation factored for both the method blank measurements (10) and the instrument blank measurements (3). A majority of the elements had LOD and LOQ in the single digit $\mu \mathrm{g} / \mathrm{L}$ range or better. Elements, such as Al, Si, and Fe had higher limits and ranged between 14 to $36 \mu \mathrm{g} / \mathrm{L}$. This was to be expected since these elements are quite common in the environment or present common interferences that require the use of less sensitive isotopes (e.g. ${ }^{57} \mathrm{Fe}, 2.19 \%$ abundance, instead of ${ }^{56} \mathrm{Fe}, 91.66 \%$ abundance, to avoid the common argon interferences at $\left.\mathrm{m} / \mathrm{z} 56\right) .{ }^{13}$ The low LOD and LOQ corroborated that this ICP-MS digestion method is fit for the purpose of determining trace concentrations of 34 possible IGSR elements in modern primers, even when these are present at trace levels.

Bias and recovery were calculated to evaluate the trueness of the developed method. Trueness is a measurement of how close the results obtained from samples subjected to the entire method are to the reference, or "true", value spiked into the samples. ${ }^{11}$ The results of this study indicated that all elements had less than $10 \%$ bias, except for aluminum and silicon; the concentrations of these two elements are affected by common sources of contamination and instrumental interferences. Calculated percent recovery ranged between 90-110\%, except for silicon, which exhibited a recovery between $113-122 \%$; this is most likely due to interference with nitrogen at mass/charge of 28 , which is a known isobaric interference with the most abundant $\mathrm{Si}$ isotope $\left({ }^{28} \mathrm{Si}\right)$, but sensitivity was compromised by using the alternative less abundant isotope $\left.{ }^{29} \mathrm{Si}\right) .{ }^{13}$

Precision was evaluated at two different levels (i.e., $25 \mu \mathrm{g} / \mathrm{L}$ and $50 \mu \mathrm{g} / \mathrm{L}$ ) to assess repeatability and reproducibility of the method over time and to determine the variability in the results. Figures 3 and 4 show the bar graphs comparing the \%RD of percent recoveries for each element at the two concentration levels $(25 \mu \mathrm{g} / \mathrm{L}$ and $50 \mu \mathrm{g} / \mathrm{L}$; the bars represent and compare the inter and intra-day variability). When validating this method, the same analyst would prepare all the samples within the same run (intraday variability), but the analyst that prepared the samples could change between different days (interday variability). Therefore, in addition to normal 
instrument variation between days, analyst variation could also be a source of variability. Sample variability was acceptable with an intraday RSD around 5\% or lower and interday RSD around $15 \%$, with none over $20 \%$. The figures of merit assessed the feasibility of the validated method for the analysis of the inorganic components on GSR over time. 
Table 4: Figures of merit for the validation study of the acid digestion- ICP-MS method for analysis of gunshot residues from different primer in-house standards.

\begin{tabular}{|c|c|c|c|c|c|c|c|}
\hline \multirow[t]{2}{*}{ Analyte } & \multirow{2}{*}{$\begin{array}{c}\text { LOD } \\
(\mu \mathrm{g} / \mathrm{L})\end{array}$} & \multirow{2}{*}{$\begin{array}{c}\text { LOQ } \\
(\mu \mathrm{g} / \mathrm{L})\end{array}$} & \multirow{2}{*}{$\begin{array}{c}\mathbf{R}^{2} \\
\text { linear range }\end{array}$} & \multicolumn{2}{|c|}{ Percent recovery $(\%)$} & \multicolumn{2}{|c|}{ Bias $(\%)$} \\
\hline & & & & $25 \mu \mathrm{g} / \mathrm{L}$ & $50 \mu \mathrm{g} / \mathrm{L}$ & $25 \mu \mathrm{g} / \mathrm{L}$ & $50 \mu \mathrm{g} / \mathrm{L}$ \\
\hline $7 \mathrm{Li}$ & 0.08 & 0.26 & 0.9996 & $101.2 \pm 1.3$ & $100.6 \pm 1.6$ & $1.2 \pm 0.015$ & $0.6 \pm 0.009$ \\
\hline 9 Be & 0.02 & 0.06 & 0.9996 & $99.6 \pm 1.5$ & $100.5 \pm 0.8$ & $-0.4 \pm 0.007$ & $0.5 \pm 0.004$ \\
\hline $11 \mathrm{~B}$ & 0.16 & 0.54 & 0.9995 & $101.2 \pm 4.3$ & $99.1 \pm 1.6$ & $1.2 \pm 0.053$ & $-0.9 \pm 0.015$ \\
\hline $23 \mathrm{Na}$ & 2.31 & 7.71 & 0.9996 & $107.1 \pm 2.8$ & $103.6 \pm 1.6$ & $7.1 \pm 0.182$ & $3.6 \pm 0.055$ \\
\hline $24 \mathrm{Mg}$ & 0.23 & 0.78 & 0.9996 & $105.9 \pm 1.5$ & $103.5 \pm 0.9$ & $5.9 \pm 0.084$ & $3.5 \pm 0.031$ \\
\hline $27 \mathrm{Al}$ & 10.76 & 35.87 & 0.9989 & $111.6 \pm 2.1$ & $107.3 \pm 1.7$ & $11.6 \pm 0.217$ & $7.3 \pm 0.117$ \\
\hline $28 \mathrm{Si}$ & 4.56 & 15.21 & 0.9992 & $119.9 \pm 2.8$ & $115.0 \pm 1.7$ & $19.9 \pm 0.472$ & $15.0 \pm 0.216$ \\
\hline $39 \mathrm{~K}$ & 2.50 & 8.35 & 0.9995 & $102.4 \pm 3.1$ & $101.4 \pm 2.8$ & $2.4 \pm 0.074$ & $1.4 \pm 0.040$ \\
\hline $44 \mathrm{Ca}$ & 0.48 & 1.59 & 0.9988 & $105.7 \pm 2.5$ & $104.1 \pm 1.8$ & $5.7 \pm 0.134$ & $4.1 \pm 0.073$ \\
\hline $47 \mathrm{Ti}$ & 0.05 & 0.17 & 0.9996 & $102.8 \pm 2.1$ & $101.6 \pm 0.7$ & $2.8 \pm 0.057$ & $1.6 \pm 0.011$ \\
\hline $51 \mathrm{~V}$ & 0.02 & 0.06 & 0.9996 & $101.2 \pm 0.6$ & $100.6 \pm 0.5$ & $1.2 \pm 0.007$ & $0.6 \pm 0.003$ \\
\hline $52 \mathrm{Cr}$ & 0.23 & 0.77 & 0.9997 & $101.3 \pm 0.3$ & $101.2 \pm 0.4$ & $1.3 \pm 0.004$ & $1.2 \pm 0.005$ \\
\hline $55 \mathrm{Mn}$ & 0.04 & 0.13 & 0.9996 & $100.0 \pm 2.6$ & $100.4 \pm 0.8$ & $-0.1 \pm 0.003$ & $0.4 \pm 0.003$ \\
\hline $57 \mathrm{Fe}$ & 4.33 & 14.43 & 0.9976 & $106.0 \pm 4.0$ & $101.8 \pm 1.2$ & $6.0 \pm 0.229$ & $1.8 \pm 0.020$ \\
\hline $60 \mathrm{Ni}$ & 0.17 & 0.58 & 0.9996 & $98.8 \pm 2.4$ & $99.5 \pm 0.7$ & $-1.2 \pm 0.028$ & $-0.5 \pm 0.004$ \\
\hline $63 \mathrm{Cu}$ & 0.36 & 1.21 & 0.9997 & $101.2 \pm 4.3$ & $100.6 \pm 1.0$ & $1.2 \pm 0.049$ & $0.6 \pm 0.006$ \\
\hline $66 \mathrm{Zn}$ & 1.08 & 3.59 & 0.9969 & $98.7 \pm 3.7$ & $99.0 \pm 1.7$ & $-1.3 \pm 0.048$ & $-1.0 \pm 0.018$ \\
\hline $71 \mathrm{Ga}$ & 0.01 & 0.03 & 0.9998 & $100.6 \pm 0.5$ & $100.5 \pm 0.4$ & $0.6 \pm 0.003$ & $0.5 \pm 0.002$ \\
\hline $85 \mathrm{Rb}$ & 0.03 & 0.10 & 0.9998 & $101.6 \pm 0.5$ & $101.6 \pm 0.2$ & $1.6 \pm 0.008$ & $1.6 \pm 0.004$ \\
\hline $88 \mathrm{Sr}$ & 0.01 & 0.04 & 0.9996 & $101.3 \pm 0.4$ & $101.1 \pm 0.3$ & $1.3 \pm 0.006$ & $1.1 \pm 0.004$ \\
\hline $90 \mathrm{Zr}$ & 0.02 & 0.06 & 0.9997 & $99.6 \pm 2.4$ & $100.6 \pm 0.7$ & $-0.4 \pm 0.011$ & $0.6 \pm 0.004$ \\
\hline $93 \mathrm{Nb}$ & 0.06 & 0.21 & 0.9997 & $97.6 \pm 2.4$ & $98.3 \pm 0.6$ & $-2.4 \pm 0.060$ & $-1.7 \pm 0.010$ \\
\hline 97 Mo & 0.10 & 0.35 & 0.9996 & $99.9 \pm 2.5$ & $100.8 \pm 0.8$ & $-0.1 \pm 0.002$ & $0.8 \pm 0.006$ \\
\hline $118 \mathrm{Sn}$ & 0.09 & 0.28 & 0.9990 & $101.3 \pm 2.8$ & $105.6 \pm 7.4$ & $1.3 \pm 0.037$ & $5.6 \pm 0.392$ \\
\hline $121 \mathrm{Sb}$ & 0.12 & 0.40 & 0.9993 & $98.5 \pm 2.5$ & $100.2 \pm 0.7$ & $-1.5 \pm 0.037$ & $0.2 \pm 0.002$ \\
\hline $133 \mathrm{Cs}$ & 0.01 & 0.05 & 0.9989 & $102.0 \pm 0.4$ & $102.6 \pm 0.3$ & $2.0 \pm 0.008$ & $2.6 \pm 0.007$ \\
\hline $137 \mathrm{Ba}$ & 0.11 & 0.35 & 0.9995 & $101.8 \pm 0.8$ & $102.8 \pm 0.8$ & $1.8 \pm 0.014$ & $2.8 \pm 0.022$ \\
\hline $157 \mathrm{Gd}$ & 0.01 & 0.03 & 0.9991 & $103.2 \pm 0.6$ & $105.6 \pm 0.5$ & $3.2 \pm 0.018$ & $5.6 \pm 0.025$ \\
\hline $178 \mathrm{Hf}$ & 0.01 & 0.02 & 0.9994 & $104.5 \pm 0.8$ & $107.3 \pm 0.4$ & $4.5 \pm 0.036$ & $7.3 \pm 0.027$ \\
\hline $181 \mathrm{Ta}$ & 0.01 & 0.03 & 0.9992 & $92.1 \pm 1.2$ & $99.8 \pm 0.7$ & $-7.9 \pm 0.101$ & $-0.2 \pm 0.002$ \\
\hline $182 \mathrm{~W}$ & 0.44 & 1.45 & 0.9991 & $103.1 \pm 1.4$ & $105.1 \pm 0.5$ & $3.1 \pm 0.042$ & $5.1 \pm 0.024$ \\
\hline $195 \mathrm{Pt}$ & 0.01 & 0.03 & 0.9993 & $103.3 \pm 1.0$ & $106.8 \pm 0.4$ & $3.3 \pm 0.031$ & $6.8 \pm 0.027$ \\
\hline $208 \mathrm{~Pb}$ & 0.11 & 0.38 & 0.9995 & $99.3 \pm 0.7$ & $101.6 \pm 0.6$ & $-0.7 \pm 0.005$ & $1.6 \pm 0.009$ \\
\hline $209 \mathrm{Bi}$ & 0.01 & 0.02 & 0.9993 & $98.3 \pm 0.8$ & $103.4 \pm 0.5$ & $-1.7 \pm 0.013$ & $3.4 \pm 0.017$ \\
\hline
\end{tabular}




\title{
Precision of the analytes for the $25 \mu \mathrm{g} / \mathrm{L}$ spiked solutions
}

\author{
- Interday Precision (RSD\%) \$Intraday Precision (RSD\%)
}

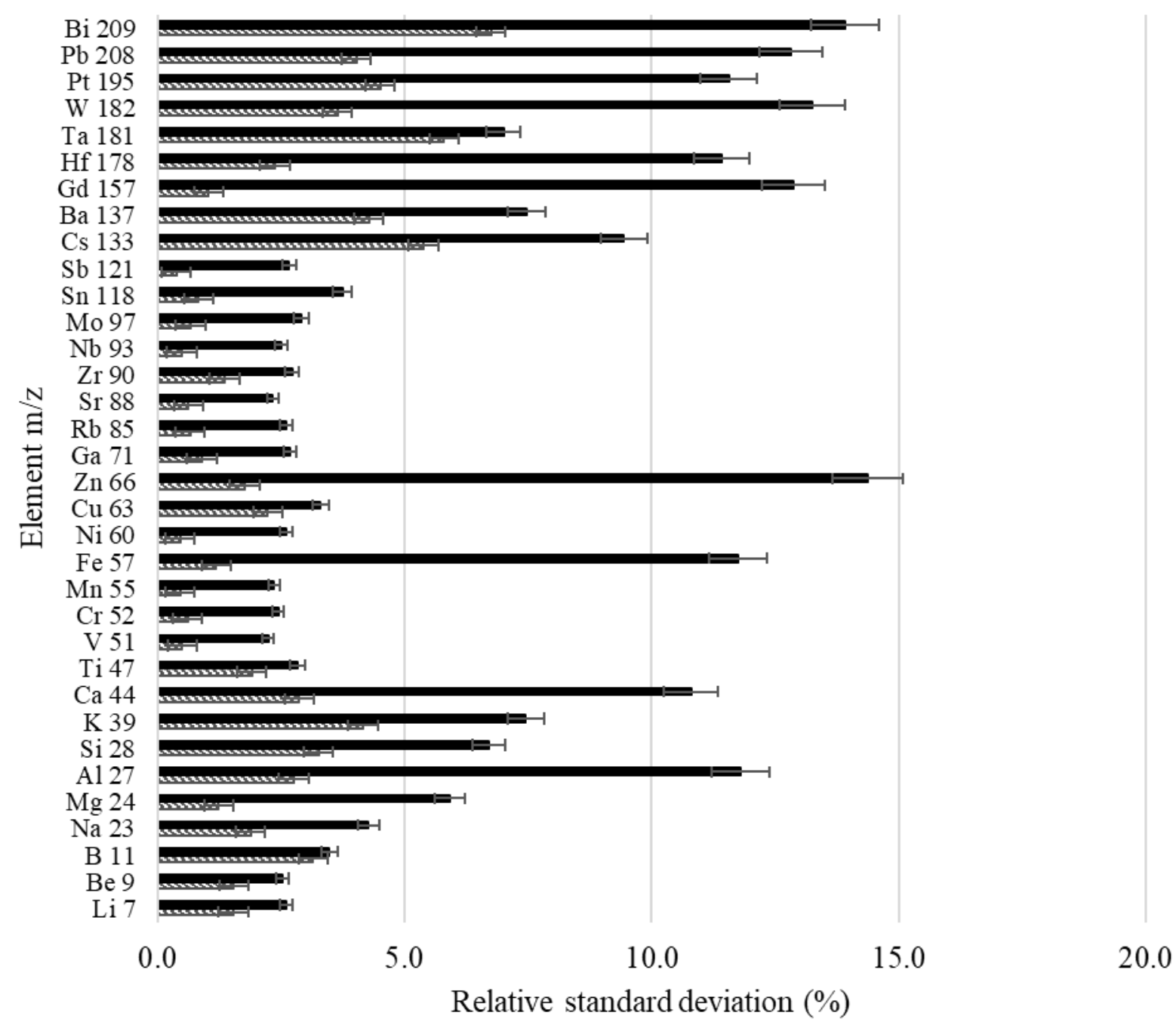

Figure 3: Chart displaying the inter- and intraday variability of the method at $25 \mu \mathrm{g} / \mathrm{L}$. Each analyte was monitored on four different day with 6-9 replicate measurements each day. 


\section{Precision of the analytes for the $50 \mu \mathrm{g} / \mathrm{L}$ spiked solutions}

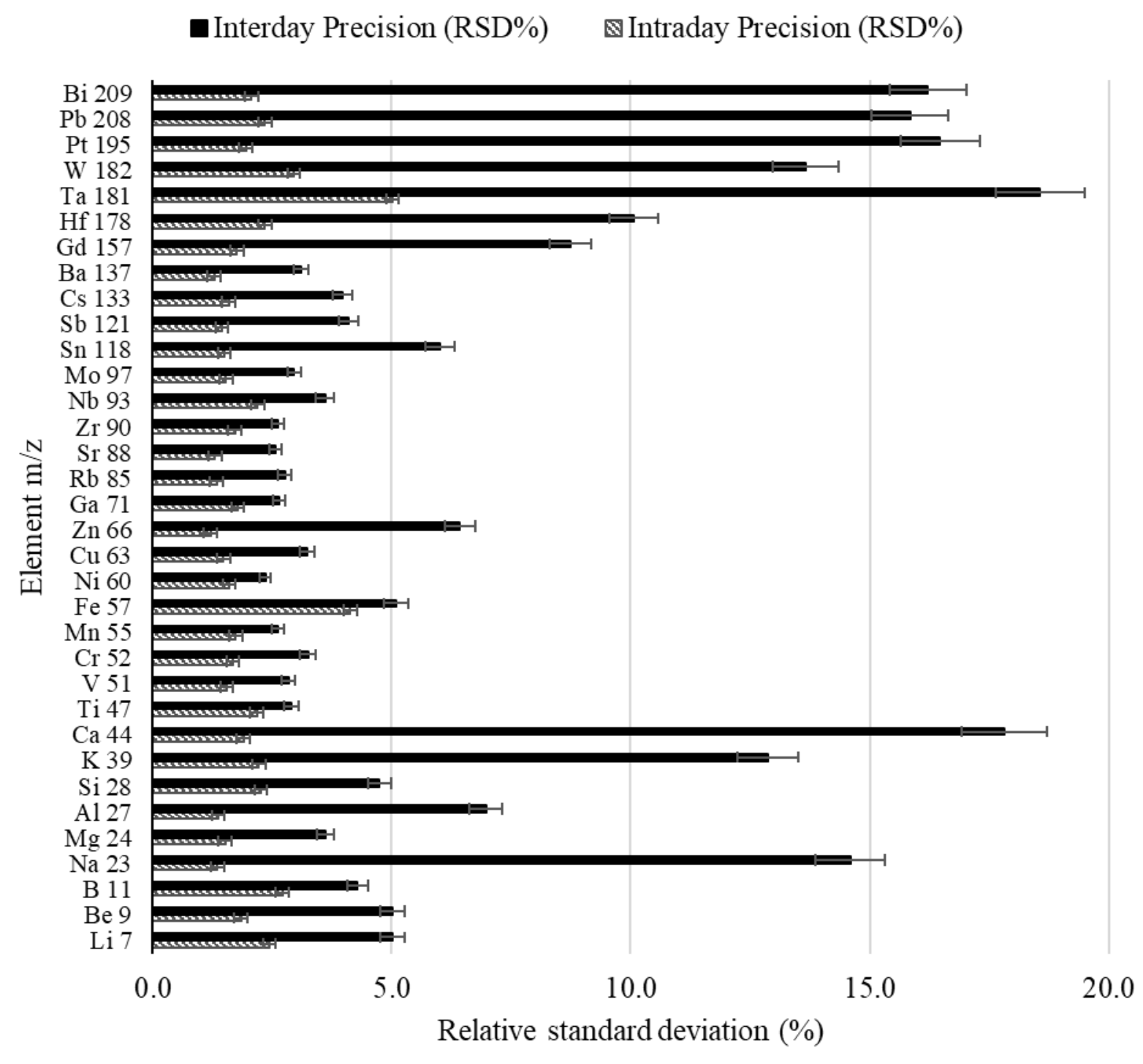

Figure 4: Chart displaying the inter- and intraday variability of the method at $50 \mu \mathrm{g} / \mathrm{L}$. Each analyte was monitored on four different day with 6-9 replicate measurements each day. 


\subsection{Conclusion}

The objective of a validation procedure is to demonstrate that it is suitable for its intended purpose. This is critical in analytical chemistry and forensic sciences, where the scientific validity of a method should be unambiguously established.

In this study, the characterization of the elemental composition of gunshot residues from primer served as a "ground truth" of the profiles expected during the transfer and persistence of GSR of hands of a shooter. The accurate characterization of these ammunition was critical for the several goals of this study: 1) the utility of tailored made standards (chapter 2), 2) the validation of emerging methods (chapter 3) and 3) the interpretation of GSR evidence (chapter 4). Therefore, a thorough validation of the characterization of GSR microparticles by acid digestion-ICP-MS was a fundamental first step in this research.

After completing the validation of the method through ruggedness testing and evaluation of figures of merit, the digestion protocol for the detection of 34 possible IGSR element was deemed successful and fit for purpose. The results from the Plackett-Burman experimental design served as the basis to identify the conditions that were critical to control in the digestion process. All the main factors (acid concertation, time between preparation and digestions, and time between digestion and analysis) were written into a standard operating procedure and monitored closely using spiked samples of acceptable reference materials. Also, correlation between the different factors was not observed, so the results of the experimental design showed valid. Therefore, an efficient digestion method was written based on the results of the.

The validation of the method reports selectivity for elements of interest within acceptable range for ICP-MS analysis. ${ }^{10}$ The linear working range for all 34 elements of interest provided $\mathrm{R}^{2}$ values of .99 or better. LOD and LOQ were found to be in the single ppb range, apart from $\mathrm{Al}, \mathrm{Si}$, and Fe which were still in the low ppb range. These results provided confidence that the method could detect and quantify trace elements in the standards at anticipated low concentration levels.

In addition, the trueness and precision of the method was successfully assessed. The trueness was evaluated though bias and recovery. All the elements, except $\mathrm{Al}$ and $\mathrm{Si}$, had less than $10 \%$ bias and exhibited recoveries between 90-110\%. Precision examined the interday and intraday variability of the recovered analytes by calculating the RSD\% at two different analyte concentrations. The intraday variability was around 5\% or lower, and the interday was around $15 \%$ with no elements being over $20 \%$. These results provided confidence that the concentrations in the 
tailor-made IGSR standards could be accurately and reproducibly monitored over the stability study.

After ruggedness testing and full validation, this method was deemed "fit for purpose" and continued to be used for the bulk stability study discussed in the next chapter. The low LOQ and tight recovery values provided an accurate assessment of the analyte concentrations for the bulk digestion of the standard. These results provided of clear picture that the method could be used to assess concentration stability over time for the tailor-made IGSR standards.

\subsection{References}

(1) Menking-Hoggatt, K.; Martinez, C.; Vander Pyl, C.; Heller, E.; Pollock, E. “Chip”; Arroyo, L.; Trejos, T. Development of Tailor-Made Inorganic Gunshot Residue (IGSR) Microparticle Standards and Characterization with a Multi-Technique Approach. Talanta 2021. https://doi.org/10.1016/j.talanta.2020.121984.

(2) Hogg, S. R.; Hunter, B. C.; Smith, R. W. Elemental Characterization and Discrimination of Nontoxic Ammunition Using Scanning Electron Microscopy with Energy Dispersive X-Ray Analysis and Principal Components Analysis. J. Forensic Sci. 2016, 61 (1), 35-42. https://doi.org/10.1111/1556-4029.12881.

(3) Martiny, A.; Campos, A. P. C.; Sader, M. S.; Pinto, M. A. L. SEM/EDS Analysis and Characterization of Gunshot Residues from Brazilian Lead-Free Ammunition. Forensic Sci. Int. 2008, 177 (1), E9-E17. https://doi.org/10.1016/j.forsciint.2007.07.005.

(4) Vanini, G.; Souza, R. M.; Destefani, C. A.; Merlo, B. B.; Piorotti, T. M.; de Castro, E. V. R.; Carneiro, M.; Romao, W. Analysis of Gunshot Residues Produced by .38 Caliber Handguns Using Inductively Coupled Plasma-Optical Emission Spectroscopy (ICP OES). Microchem. J. 2014, 115, 106-112. https://doi.org/10.1016/j.microc.2014.03.003.

(5) Abrego, Z.; Grijalba, N.; Unceta, N.; Maguregui, M.; Sanchez, A.; Fernandez-Isla, A.;

Goicolea, M. A.; Barrio, R. J. A Novel Method for the Identification of Inorganic and Organic Gunshot Residue Particles of Lead-Free Ammunitions from the Hands of Shooters Using Scanning Laser Ablation-ICPMS and Raman Micro-Spectroscopy. Analyst 2014, 139 (23), 6232-6241. https://doi.org/10.1039/c4an01051e.

(6) Mason, K.; Wuhrer, R. Detection and Characterization of Heavy-Metal-Free (HMF) Gunshot Residues Using CL, EDS and Raman Together with XRD. Mircoscopic 
Microanal. 2018, 24 (1), 1174-1175. https://doi.org/10.1017/S1431927618006359.

(7) Donghi, M.; Mason, K.; Romolo, F. S. Detecting Gunshot Residue from Sellier \& Bellot Nontox Heavy Metal-Free Primer by in Situ Cathodoluminescence. J. Forensic Sci. 2019, 64 (6), 1658-1667. https://doi.org/10.1111/1556-4029.14110.

(8) Fambro, L. A.; Miller, E. T.; Vanderbos, D. D. Characterization of Lead-Free Gunshot Residue Analogs. Anal. Methods 2016, 8, 3132-3139.

https://doi.org/10.1039/c6ay00725b.

(9) Standard Guide for Gunshot Residue Analysis by Scanning Electron Microscopy / Energy Dispersive X-Ray Spectrometry 1; West Conshohocken, PA, 2009.

https://doi.org/10.1520/E1588-20.2.

(10) Agency, U. S. E. P. Method 6020B (SW-846): Inductively Coupled Plasma-Mass Spectrometry. Washington, DC 2014, p 33.

(11) Eurachem Guide: The Fitness for Purpose of Analytical Methods - A Laboratory Guide to Method Validation and Related Topics, 2nd ed.; Magnusson, B., Ornemark, U., Eds.; 2014.

(12) ASTM E30 Committee, Standard Practice for Conducting Rugedness Tests. ASTM E 1169-18. ASTM International: West Conshohocken, PA 2018, p 13.

(13) May, T. W.; Wiedmeyer, R. H. A Table of Polyatomic Interferences in ICP-MS. At. Spectrosc. 1998, 19 (5), 150-155. 


\section{Section 4}
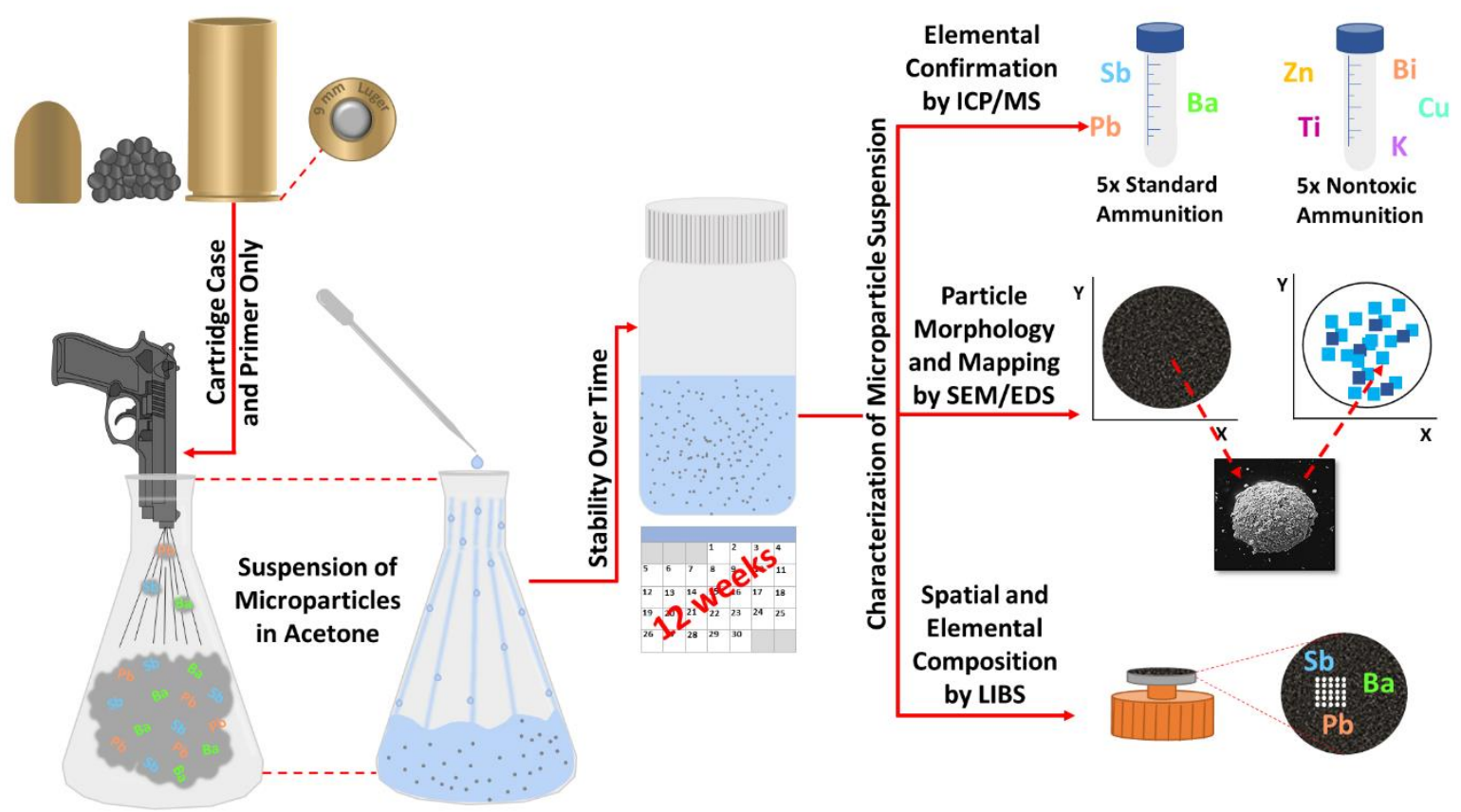

Chapter 2

Multi-Technique Approach to Characterization of the Tailor-Made IGSR Suspensions 


\section{Chapter 2. Multi-Technique Approach to Characterization of the Tailor-Made IGSR Suspensions}

\subsection{Overview}

The forensic analysis of inorganic gunshot residues (IGSR) involves analytical measurements from samples taken from skin and other substrates. The standard practice for IGSR analysis recommends the use of Scanning Electron Microscopy-Energy Dispersive X-Ray Spectroscopy (SEM-EDS) to identify the gunshot residues using combined information of the particle's morphology and elemental composition. However, the current deficit on IGSR standard reference materials (SRM) limits the optimization of SEM-EDS for modern, lead-free ammunition and the development of emerging analytical techniques. This study aims to enhance existing capabilities by producing tailor-made microparticle suspensions that can be used for the quality control of GSR analysis, validation of existing and emerging methods, interlaboratory testing, and systematic transfer and persistence studies. To fill this gap, IGSR microparticle standards were developed by discharging various leaded and lead-free primers under controlled conditions and creating suspensions in an organic medium, then evaluated for homogeneity and stability of morphology and elemental composition. The IGSR microparticles suspensions were evaluated by three analytical techniques-SEM-EDS, Laser-Induced Breakdown Spectroscopy (LIBS) and Inductively Coupled Plasma-Mass Spectrometry (ICP-MS) - to characterize the elemental composition and particle morphology. The ICP-MS digestion method was validated for these novel IGSR microparticle suspensions, and figures of merit and ruggedness testing are reported. The standard demonstrated stability in its dry and suspension forms, providing versatility for use in multiple types of analytical methods and substrates. This research is anticipated to assist forensic and environmental scientists by providing IGSR standards that can strengthen research, expand access to new detection techniques, and enhance laboratories' cross-validation and quality assurance.

The following chapter is an adaptation of a previously published article (O2021:

K Menking-Hoggatt, C Martinez, C Vander Pyl, E Heller, E Pollock, L Arroyo, and T Trejos. Development of Tailor-Made Inorganic Gunshot Residue (IGSR) Microparticle Standards and Characterization with a Multi-technique Approach. Talanta. Published online December 2020, hardcopy publication April 2021, 225. https://doi.org/10.1016/j.talanta.2020.121984 


\subsection{Introduction}

Gunshot residue (GSR) is a common form of trace evidence, typically analyzed by Scanning Electron Microscopy-Energy Dispersive X-ray Spectroscopy (SEM-EDS), for its elemental composition and morphology. ${ }^{1}$ However, regardless of its scientific reliability, current practice and research in this field have identified the needs for the development of IGSR standard materials that can represent the diversity of modern ammunition and the assessment of complementary methods of analysis. ${ }^{2}$

Crime laboratories currently test for Inorganic GSR (IGSR) because it is a valuable form of trace evidence that can aid in investigation and crime reconstruction. During a firearm discharge incident, a plume of residues is ejected from the firearm and deposited as microscopic IGSR on surfaces in the vicinity of the event. ${ }^{3,4}$ The particles vary in elemental composition depending on the type of ammunition. For instance, leaded ammunition typically contains lead, barium, and antimony. In contrast, lead-free ammunition varies more in composition and can include elements such as potassium, calcium, titanium, zinc, copper, and strontium, among others.$^{5-12}$ Regardless of the type of ammunition used, the particles are created under high temperatures and pressures in a fraction of a second. ${ }^{13,14}$ After the particles exit the firearm, they undergo rapid cooling during their flight trajectory where cohesive forces typically produce spheroid microparticles (often between $0.5-10 \mu \mathrm{m}) .^{3,15-17}$ These IGSR microparticles cannot be seen by the naked eye, but possess the ability to undergo primary, secondary, and tertiary transfer processes between surfaces. ${ }^{16,18-21}$ This attribute of IGSR makes it valuable in forensics because it can assist in understanding not only the source (e.g. is it GSR rather than other trace?), but also the relationship between the person(s) of interest and the activities connecting the use of a firearm to an event.

Over the years, research has been designed to assess the capabilities of multiple types of instrumentation for IGSR detection focusing mainly on their sensitivity and selectivity. The earliest types were sensitive instruments such as Neutron Activation Analysis (NAA) and Atomic Absorption Spectroscopy (AAS). ${ }^{22-26}$ Inductively Coupled Plasma (ICP) coupled to either Optical Emission Spectrometry (OES) or Mass Spectrometry (MS) has also been studied to quantitatively detect the trace amounts of inorganic elements. ${ }^{27-29}$ While all these methods are scientifically valid, they are destructive to the evidence and provide no information about the morphology of the IGSR microparticles. Time of Flight-Secondary Ion Mass Spectrometry (TOF-SIMS) and Focused Ion 
Beam (FIB) analysis provides composition and morphological information with better resolution and limits of detection than SEM-EDS. ${ }^{17,30-33}$

However, SEM-EDS is by far the most widely available and confirmatory tool for IGSR detection. ${ }^{1,34}$ SEM-EDS is recognized as the gold standard for IGSR identification due to its ability for imaging, counting number of particles, and its capacity to conduct single particle elemental analysis on GSR particles as small as $0.5 \mu \mathrm{m}$, along with the availability at crime laboratories and versatility of SEM-EDS analysis for trace evidence in general. The sole use of SEM-EDS for IGSR analysis may become challenging with some modern ammunition, particularly in those cases with lead-free gunshot residues. For instance, lead-free ammunition produces IGSR that lacks heavy elements such as lead, barium, and antimony and in some formulations, the generated IGSR particles may contain only low atomic number elements, such as potassium. ${ }^{1}$ These low atomic particles are more difficult to detect by backscatter imaging and to differentiate from other background materials during the automated discovery analysis.

Another limitation of SEM-EDS is that the method is relatively time-consuming. ${ }^{35}$ As a result, there is a growing interest to find complementary methods that can help with on-site detection and more efficient and comprehensive case management in the laboratory. For instance, faster methods can help to make informed decisions about both collection at the crime scene and analysis at the laboratory by quickly screening the evidence and limiting the number of items requiring confirmatory analysis by SEM-EDS. Moreover, having rapid testing available can allow the incorporation of more holistic approaches, such as including additional GSR samples collected from nostrils, clothing, and surfaces from the scene. ${ }^{36}$ These types of samples are sometimes limited due to the time it would take to analyze multiple item types per case, which would further stress the backlogs in the laboratory using SEM-EDS.

In response to these limitations, novel approaches using Raman Spectroscopy, Laser Induced Breakdown Spectroscopy (LIBS), electrochemistry, Raman, and mass spectrometry have been proposed to complement SEM-EDS analysis. ${ }^{8,28-30,37-57}$ Of particular interest to the study presented here is LIBS, which has emerged as viable screening tool for IGSR detection due to the many benefits, such as ease of use, relative low cost, rapid analysis ( $\sim 1$ min per stub), no sample preparation needed, portability, simultaneous multielemental information, spatial information on the micrometer scale, and negligible sample destruction that allows further SEM-EDS confirmation on the same sample. ${ }^{8,37,56,57,38,49-55}$ Adopting more thorough complementary 
approaches can increase reliability and efficiency, providing valuable data and a streamlined process with fewer errors and delays.

In addition, another need identified in the GSR discipline is increasing the availability of standard reference materials that mimic modern ammunition compositions. ${ }^{2,58,59}$ Authentic GSR samples are useful for purposes of estimating error rates and population information, but due to the stochastic nature of a firing event, these types of residues are not feasible as routine quality control standards with known particle count, elemental composition, and morphological features. $1,22,39,53,57,60$

Instead, SEM-EDS examiners have access to a commercial synthetic standard specifically created for the detection of IGSR from leaded ammunition. The standard is used as quality control, with known particle count, size, and location. Although the substrate surface is not identical to the analysis surface of a typical GSR collection stub, these standards have demonstrated to fit for purpose and are a valuable asset as inter-laboratory testing materials. ${ }^{34,60-62}$ Nonetheless, the standard only contains $\mathrm{Pb}, \mathrm{Ba}$ and $\mathrm{Sb}$, and cannot be applied to the testing of other emerging methods that need more versatile and consumable configurations.

A common need during the evaluation of analytical techniques is to rely on quality control matrix-matched laboratory standards. Although standard reference materials such as the Planotech are widely used in SEM-EDS, the expansion to complementary standard reference material (SRM) with more variable elemental compositions may become useful in the future. ${ }^{34}$ Moreover, more versatile standards that allow for dry and liquid forms can also help researchers assess novel technologies more effectively. An SRM such as this could facilitate interlaboratory testing and cross-validation of techniques and open doors to research on alternative analytical tools and better understanding of the fundamental mechanisms of deposition, transfer and persistence of IGSR.

This study aimed to fulfill the existing gap on IGSR standards by developing and validating novel IGSR microparticle suspension with characterized elemental composition and morphology in a variety of elemental combinations from modern primers. The suspensions were designed to be used as liquid or dry configurations, as reference material or quality control samples for various methods for IGSR detection. The purpose of these standards is to provide the forensic community with multiuse standard materials that can be deposited, imaged, and analyzed in the same manner as typical IGSR. Moreover, the use of the suspensions is envisioned for expanded use in many other environmental and occupational safety applications, such as the monitoring of airborne 
particulate in shooting ranges and studies on the fate and persistence of heavy metal gunshot residues in the environment.

\subsection{Materials and Methods}

\subsubsection{Collection of IGSR Microparticle Samples}

A novel method was developed to collect IGSR microparticle samples. To accomplish this, the IGSR samples were created by using a piece of ammunition that contained only a cartridge case and a primer with no propellant or bullet. The cartridge case and primer ammunition was seated in the barrel of a 9mm semi-automatic pistol (Springfield Armory, IL) and the chamber was closed. If the ammunition was loaded by the manufacturer, the bullet and propellant were removed, and the cartridge case and primer remained intact. Pressurized air was used to remove any propellant left in the cartridge case. All ammunition was similarly discharged by placing the end of the barrel (muzzle) into a clean 2 L Nalgene ${ }^{\circledR}$ Erlenmeyer flask (Thermo Scientific, NY) and pulling the trigger to release the firing pin, which discharged the primer. The mouth of the flask was immediately covered with a piece of Parafilm (Pechiney, WI) and allowed to settle for $1 \mathrm{~min}$ before proceeding.

The flask was rinsed five times with approximately $100 \mathrm{~mL}$ of Optima grade acetone (Fisher Chemical, NJ), and the rinse was collected in a Nalgene ${ }^{\circledR}$ high-density polypropylene container with a screw-on lid (Thermo Scientific, NY). A blank sample was also collected by filling the same type of container with approximately $100 \mathrm{~mL}$ of Optima grade acetone (same lot). The blank sample was used to monitor any possible contamination that might originate from the solvent or the container. The entire collection was performed under a negative pressure hood. Appropriate laboratory personal protective equipment was worn at all times. Once collected, the capped stock suspensions were stored at room temperature when not in use. All ten of the different primers used in this study were collected in triplicate (i.e. replicate A, B, and C) using the process described. This resulted in a total of 30 separate stock IGSR microparticles suspensions. The firearm was cleaned with a solvent (Hoppe's No. 9; Cody Overland Park, KS), wiped with cotton swatches until the residues were removed, and then allowed to dry between each primer change to minimize memory effects. A summary of the different primer specifications used in this study can be found in Table 1 . 
Table 1: List of the different primer types collected for the study.

\begin{tabular}{|c|c|c|c|c|}
\hline Brand & $\begin{array}{l}\text { Abbreviation } \\
\text { for this study }\end{array}$ & $\begin{array}{l}\text { Lead- } \\
\text { free }\end{array}$ & $\begin{array}{l}\text { Manufacture } \\
\text {-loaded }\end{array}$ & Specifications \\
\hline Federal & FED & no & no & $\begin{array}{l}\text { Small pistol primers for standard pistol loads for } \\
\text { loading } 9 \mathrm{~mm}\end{array}$ \\
\hline Remington & REM & no & no & $\begin{array}{l}\text { No. } 11 / 2 \text { Kleanbore }{ }^{\circledR} \text { small pistol primers for loading } \\
9 \mathrm{~mm}\end{array}$ \\
\hline $\begin{array}{l}\text { Sellier \& } \\
\text { Bellot }\end{array}$ & $\mathrm{SAB}$ & no & no & $\begin{array}{l}\text { Non-corrosive, non-erosive } 4,4 \text { SR BOXER primers } \\
\text { for loading } 9 \mathrm{~mm}\end{array}$ \\
\hline TulAmmo & TUL & no & no & $\begin{array}{l}\text { Non-corrosive KVB-9 Staynless } ® \text { small pistol primers } \\
\text { for loading } 9 \mathrm{~mm}\end{array}$ \\
\hline Winchester & WIN & no & no & $\begin{array}{l}\text { Small pistol primers for standard pistol loads for } \\
\text { loading } 9 \mathrm{~mm}\end{array}$ \\
\hline $\begin{array}{l}\text { CCI } \\
\text { non-leaded }\end{array}$ & $\mathrm{CCX}$ & yes & no & Lead-free small pistol primer for loading $9 \mathrm{~mm}$ \\
\hline Fiocchi & FIO & yes & no & $\begin{array}{l}\text { Heavy metal and zinc free small pistol primers for } \\
\text { loading } 9 \mathrm{~mm}\end{array}$ \\
\hline Hevi-shot & HEV & yes & yes & Frangible non-leaded 9mm 100 grain bullet \\
\hline $\begin{array}{l}\text { Inceptor } \\
\text { RNPTM }\end{array}$ & INC & yes & yes & $\begin{array}{l}\text { RNP } 65 \text { grain frangible copper-polymer matrix bullet } \\
\text { for loading } 9 \mathrm{~mm}\end{array}$ \\
\hline $\begin{array}{l}\text { SYNTECH } \\
\text { Federal }\end{array}$ & SYN & yes & yes & 9mm Luger TSJ 124 grain bullet \\
\hline
\end{tabular}

\subsubsection{ICP-MS Analysis on IGSR Microparticle Samples}

\subsubsection{Stability Study}

Sample preparation for ICP-MS began with digestion and then dilution of the IGSR microparticles. The acetone samples containing the microparticles were thoroughly vortexed for 1 min. An aliquot of $100 \mu \mathrm{L}$ of the microparticle samples was added to $50 \mathrm{~mL}$ digestion tubes (Environmental Express, SC). The tubes remained uncapped for approximately $1 \mathrm{~h}$ at room temperature in a negative pressure chemical hood. Once all the acetone evaporated, $100 \mu \mathrm{L}$ of 10 $\mathrm{mg} / \mathrm{L}$ stock solutions of scandium, yttrium, and indium mix were added as internal standards and filled to $5 \mathrm{~mL}$ with $10 \%$ (v/v) Optima grade nitric acid (Fisher Chemical, NJ). Samples were placed 12 at a time in a 12-channel HotBlock dry heating block (Environmental Express, SC) set to $80^{\circ} \mathrm{C}$. The samples were refluxed for $1 \mathrm{~h}$ with polypropylene watch glass covers (Environmental Express, $\mathrm{SC})$ over the mouths of the tubes.

Each digestion set of 12 samples contained reagent blanks and spiked quality control samples to monitor any contamination and differences between digestions. The spiked quality control samples included ICP-MS standards of each of the elements of interest (34 analytes of 
interest). These samples were used as quality control to monitor recovery, bias, precision, and accuracy. The reagent blanks and the spiked quality control samples also contained the $100 \mu \mathrm{L}$ of the internal standard mix (scandium, yttrium, and indium).

After digestion, the tubes were removed from the heating block, and placed in a rack under the hood to cool while covered with the watch glasses. Once cooled, the watch glasses were carefully removed, rinsed and the volume was reconstituted to $5 \mathrm{~mL}$ with the same stock of $10 \%$ (v/v) nitric acid using a transfer pipette (Fisher Brand, PA). The samples were finally vortexed for 30 seconds before dilution.

Dilution of the samples was performed to lower the acid concentration from $10 \%(\mathrm{v} / \mathrm{v})$ to approximately $2.5 \%$, which is appropriate for ICP-MS analyses. This was accomplished by transferring $2.5 \mathrm{~mL}$ of the digested solution to round-bottom polypropylene tubes (Fisher Scientific, KY) and diluting to $10 \mathrm{~mL}$ with deionized water. The tubes were vortexed again for 30 seconds and placed in a tube rack until analysis. All the samples were prepared and digested in a negative pressure chemical hood lined with clean butcher paper, using a clean lab coat and disposable nitrile gloves to prevent contamination.

A 12-week study was designed to test the quantitative and qualitative stability of the IGSR microparticles suspensions over time at weeks $0,2,6$, and 12 . The experimental design aimed to assess the stability of the elemental composition of the IGSR microparticle reference standards suspended in the organic medium and stored in the laboratory for extended periods of time. Each sample was prepared in triplicate for ICP-MS analysis using the validated procedure described in section 3: chapter 1 and repeated at the week intervals specified.

\subsubsection{ICP-MS Instrumental Parameters}

The ICP-MS instrument (Agilent 7800, Santa Clara, CA) was equipped with a MicroMist nebulizer, a double pass quartz Scott-type spray chamber, and a quartz torch with an inner diameter of $2.5 \mathrm{~mm}$. Automated sample introduction was controlled by an autosampler (SPS 4; Santa Clara, CA) and a peristaltic pump (PeriPump; Santa Clara, CA). The operational parameters were a radio frequency of $1550 \mathrm{~W}$, an auxiliary gas flow of argon at $0.90 \mathrm{~L} / \mathrm{min}$, and a plasma gas flow of argon at $15.0 \mathrm{~L} / \mathrm{min}$. Three replicates were collected for every sample at 100 sweeps each. All the elements of interest were monitored with $\mathrm{He}$ in the collision cell, except for $\mathrm{Si}$, which was analyzed using no gas mode. Concentrations were determined using an external calibration curve with 
internal standard made from the same ICP-MS standards listed in section 2.2.1. All conditions and parameters remained constant during the validation and stability study.

\subsubsection{ICP-MS Data Analysis}

The concentrations for the analytes were reported by the ICP-MS data analysis software Mass Hunter 4.4 (version C.01.04; Santa Clara, CA) and exported for further statistical analysis using both Excel ProPlus (Microsoft 365) and JMP Pro 14.0.0. Analysis of Variance (ANOVA) and post-hoc Tukey-Kramer test were performed on the calculated concentrations to determine the stability of the elemental composition for the IGSR microparticles standards over the 12-week experiment.

\subsubsection{SEM-EDS on IGSR Microparticle Samples}

\subsubsection{SEM-EDS Samples Preparation}

The samples were prepared using the same design of carbon stubs currently used by practitioners for the collection and subsequent analysis of GSR evidence by SEM-EDS. The GSR stubs consisted of $12 \mathrm{~mm}$ diameter, double-sided adhesive carbon conductive tabs (PELCO ${ }^{\mathrm{TM}}$, TedPella, CA) mounted on a $12.7 \mathrm{~mm}$ aluminum pin mount (Zeiss specimen mounts, Al). The stubs were placed in plastic storage tubes specifically designed for aluminum pin mounts (TedPella, CA).

The IGSR microparticle samples contain numerous IGSR particles. A dilution of the sample was necessary to reduce the number of particles deposited and, in turn, the analysis time by SEM-EDS. To dilute the samples, $100 \mu \mathrm{L}$ of the IGSR microparticles standard were transferred to a $14 \mathrm{~mL}$ polypropylene round-bottom tube (Fisher Scientific, KY) and diluted with $900 \mu \mathrm{L}$ of Optima grade acetone (Fisher Chemical, NJ). A volume of the $2 \mu \mathrm{L}$ diluted suspension was then transferred to the center of a $\sim 6 \mathrm{~mm}$ circle that was scored onto the surface of a GSR carbon stub. The purpose of the scored circle was to decrease the mapping area of the stub, and therefore reduce the time of SEM-EDS analysis. The stubs were left uncapped to dry at room temperature in a negative pressure hood for at least $24 \mathrm{~h}$, allowing the acetone to evaporate while leaving the microparticles of GSR on the adhesive stub. Once the samples were dry, the stubs were stored at 
room temperature in GSR storage tubes inside cardboard boxes until analysis. The diluted samples in the $14 \mathrm{~mL}$ polypropylene round-bottom tubes were stored in a refrigerator at $4^{\circ} \mathrm{C}$ when not in use.

The deposition process of the IGSR microparticles standards was performed at 0, 6, and 12 weeks and created from one replicate of each of the collected primers. The analysis consisted of three deposits for each of the 10 primer types, which resulted in a total of 30 samples to be analyzed by SEM-EDS. A blank sample of acetone was diluted and spiked in the same manner and included in every analysis to monitor possible contamination from the acetone and/or the storage container. A synthetic quality control sample (ENFSI, Germany) was analyzed between each sample to monitor the performance of the instrument.

\subsubsection{SEM-EDS Instrumental Parameters}

SEM-EDS analysis was conducted on a JOEL 6490LV (Peabody, MA) in accordance with ASTM 1588-17 standard for the analysis of GSR. ${ }^{1}$ The manufacturer SEM user interface software was version 8.14. The instrumental parameters used during analysis and spectra collection consisted of an accelerating voltage of $25 \mathrm{kV}$, a spot size of $64 \mu \mathrm{m}$, a working distance of approximately $18 \mathrm{~mm}$, and a magnification of 1000x. A backscatter and a secondary electron detector were used to image particles, while an Oxford Instrument INCAx-sight 7623 Energy Dispersive X-ray Spectroscopy (EDS, England) detector collected elemental information about the particles of interest.

\subsubsection{SEM-EDS Data Analysis}

The SEM-EDS portion of the experiment was designed to determine if the IGSR microparticles maintained the elemental composition and morphology of the GSR particles. The analysis was automated and organized by INCAx-sight software (Microanalysis Suite Issue 17b+SP2 and Version 4.09: Concord, MA), which is designed for the management of image and spectral data. Images of represented features, and their respective spectra, were collected for each stage of the study. Particle count and size was also documented in the study. 


\subsubsection{LIBS Application of IGSR Microparticle Samples}

\subsubsection{LIBS Sample Preparation}

A volume of $100 \mu \mathrm{L}$ of the concentrated IGSR microparticle sample was deposited on a typical carbon GSR stub. This volume covered the entire surface of the stub, so LIBS analyses could be performed repeatedly on the same stub. In this study, preliminary examination of one type of standard ammunition (Remington) was conducted to assess the use of the sample as a quality control standard for daily testing of instrument performance. In addition, all ten IGSR suspensions were characterized by LIBS.

\subsubsection{2. $\quad$ LIBS Instrumental Parameters}

A J200 Tandem LIBS system (Applied Spectra, CA) equipped with a $266 \mathrm{~nm}$, high-power, Q-switched, Neodymium doped Yttrium Aluminum Garnet (Nd YAG) nanosecond laser was used for analysis. The instrument detector optics and sensor consisted of a six-channel Czerny-Turner spectrometer with a spectral range from 190 to $1040 \mathrm{~nm}$ and a Charge Coupled Device (CCD) based broadband detector, respectively. A second J200 tandem LIBS instrument almost identical to the one described was also used on a sub-set of the samples. The only difference was a second Intensified CCD (ICCD) based detector in the UV range of the spectrum was used for increased resolution and sensitivity.

The prepared samples were placed in the ablation chamber using a custom stage platform with a hole in the center that securely held the pin mount; this ensured that the surface remained even during analysis to maintain a consistent focus on the stub surface. The chamber was purged for 45 seconds with $1 \mathrm{~L} / \mathrm{min}$ of argon (ultrahigh purity, Matheson, WV) before performing analysis. The samples were examined using a previously validated micro-spot LIBS method developed by our research group. ${ }^{53,57}$

\subsubsection{LIBS Data Analysis}

The data analysis software Aurora (Applied Spectra, CA) was used to crop the collected spectra in order to isolate the desired spectral regions. Background subtraction, peak identification, and peak integration on the peaks of interest were subsequentially performed. The integrated peak 
areas were imported into Excel ProPlus (Microsoft 365) and combined into one workbook. Signalto-noise ratios (SNR) for all the emission lines of interest (I- atomic and II- ionic) - Al $394.4 \mathrm{~nm}$ (I), Al 396.1 nm (I), Ba 455.4 nm (II), Ba 493.4 nm (II), Ba 614.2 nm (II), Ba 649.7 nm (II), Bi 293.8 nm (I), Bi $412.1 \mathrm{~nm}$ (I), Bi $472.2 \mathrm{~nm}$ (I), Cu 324.7 nm (I), Cu 327.4 nm (I), Cu $515.3 \mathrm{~nm}$ (I), K 766.4 nm (I), K 769.9 nm (I), Pb 368.3 nm (I), Pb 405.8 nm (I), Sb 252.8 nm (I), Sb 259.8 nm (I), Sb 287.8 nm (I), Sb 326.7 nm (I), Si 251.4 nm (I), Si 288.1 nm (I), Si 390.5 nm (I), Sn $284.0 \mathrm{~nm}$ (I), Sn $317.5 \mathrm{~nm}$ (I), Sn $326.2 \mathrm{~nm}$ (I), Ti 334.9 nm (II), Ti $368.5 \mathrm{~nm}$ (II), Ti $375.9 \mathrm{~nm}$ (II), Ti $376.1 \mathrm{~nm}$ (II), Zn $328.2 \mathrm{~nm}$ (I), Zn $334.5 \mathrm{~nm}$ (I), Zn $481.0 \mathrm{~nm}$ (I)-were calculated by using the background of the spectrum in proximity to the peak of interest. The SNR for the most informative peaks of $\mathrm{Al}(396.1 \mathrm{~nm}), \mathrm{Ba}(455.4 \mathrm{~nm}), \mathrm{Bi} 472.2 \mathrm{~nm}(\mathrm{I}), \mathrm{Cu}(324.7 \mathrm{~nm}), \mathrm{K} 766.4 \mathrm{~nm}$ (I), Pb (405.8 nm), Sb (259.8 nm), Si $288.1 \mathrm{~nm}$ (I), Sn $326.2 \mathrm{~nm}$ (I), Ti 334.9 nm (II), and Zn 481.0 nm (I) were imported into Excel ProPlus (Microsoft 365) to perform further statistical testing.

\subsubsection{Collection of Known-Shooter Samples for Comparison}

Known-shooter samples were collected using the same firearm and the full ammunition (bullet, cartridge case, propellant, and primer). Ammunition was fired as loaded by the manufacturer. If the ammunition was specialty loaded in our department, then the same lot of Starline brass cartridge case (Sedalia, MO), Speer 115 grain total metal jacket bullet (Lewiston, ID), and Winchester 231 smokeless powder (New Haven, CT) was used. Specifics about the primers in this experiment can be found in Table 1 and follow the same abbreviation scheme as the ammunition listed. Any hand samples collected followed the previously validated method and used a typical GSR collection carbon stub. ${ }^{53,57}$

\subsection{Results and Discussion}

ICP-MS, SEM-EDS, and LIBS were strategically selected to evaluate the standards' suitability in the suspension and dry states. IGSR microparticles were collected and analyzed overtime to monitor the stability of the elemental composition and particle morphology by ICP-MS and SEM-EDS, respectively. ICP-MS was chosen as the method for characterizing the liquid standards due to its bulk quantitative capabilities, sensitivity, and selectivity. The suspensions were also deposited on GSR collection stubs and used as reference standards to optimize the detection of IGSR on the hands of shooters by LIBS and provide an additional set of complementary data 
about the elemental composition of the microparticle suspensions. ${ }^{57}$ Therefore, LIBS allowed for additional cross-validation of the standards' ruggedness during the deposition from suspension to dry form. Finally, SEM-EDS allowed for single-particle analysis, particle mapping, and testing the materials' primary application.

The SEM-EDS method was previously validated in our laboratory for routine GSR analysis, and the validation of LIBS method was previously reported by our group. ${ }^{53,57}$ However, the ICP-MS method used for the quantitative and qualitative characterization of the IGSR suspensions was first fully validated and deemed fit for purpose before performing the stability study, and the details of the results are discussed in section 3: chapter 1 of this dissertation, including results for the Plackett-Burman ruggedness test and figures of merit such as selectivity, limits of detection, limits of quantitation, trueness, and precision. ICP-MS provided a bulk and exhaustive method capable of characterizing the concentration of IGSR elements per a given volume of a suspension, while SEM-EDS offered a means to evaluate particle morphology, size, and particle count per a specific deposited amount. The combination of information derived from these two complementary methods is essential for characterizing the number of particles per volume of the suspension and absolute concentration values and uncertainty that can serve in the future for assessment of an analytical method's performance. The use of LIBS serves as a proof of concept of the applicability of these standards for the development of emerging techniques for GSR detection and for creating quality control protocols.

In this study, we developed and demonstrated the feasibility of IGSR microparticle suspensions for their use as standard materials in research and forensic laboratories. The nature of these materials can also expand to areas that involve gunshot residues outside of forensics, such as environmental monitoring. The microparticle standards were designed to offer:

(1) versatile configurations in liquid or dry forms adaptable for multiple applications,

(2) quantitative characterization of bulk concentration and the number of particles per a given volume,

(3) characterization of elemental profiles and particle morphology in the dry form/stubs,

(4) expanded microparticle IGSR standards beyond the most common $\mathrm{Pb} / \mathrm{Ba} / \mathrm{Sb}$ configuration, including various standards representative of leaded and lead-free ammunition, 
(5) large throughput of the stock suspensions to facilitate its use in quality control and interlaboratory distribution,

(6) similar composition to IGSR particles for future use in fundamental and systematic studies of deposition, transfer, and persistence of IGSR.

To achieve all those desired features, we developed a multi-technique approach for characterizing the standards, assessing their stability over time, and demonstrating its applicability in various forms and analytical tools. Of primary importance for this study was demonstrating the feasibility for future use as standard materials for standard practice (SEM-EDS), while also envisioning its application to emerging complementary methods. Likewise, we aimed to develop a reference material that is consumable, stable, and reproducible to open opportunities for fundamental studies that are currently limited due to lack of such known and characterized materials.

\subsubsection{ICP-MS Analysis and Stability Study}

ICP-MS is a sensitive analytical technique capable of detecting trace elements in samples and ideal for this research study, since we aim to provide a comprehensive characterization and quantification of the components of IGSR standards, and to correlate the elemental composition to particle counts and elemental profiles by SEM-EDS. The validation of a reference standard requires state-of-the-art instrumentation with excellent precision, sensitivity, and selectivity. However, it is important to clarify that the use of ICP-MS in this work was exclusively for validation purposes and characterization of the standards and not intended for casework, given the complicated sample preparation and destructive nature. This technique is only being utilized as a research tool and not suggested for the analysis of IGSR evidence in the crime laboratories.

The validated digestion method was applied to evaluate the stability and homogeneity of the collected IGSR microparticle suspensions in bulk form. Over the course of the stability study, the samples were digested and analyzed by ICP-MS to qualitatively and quantitatively monitor their elemental composition. Analysis of Variance (ANOVA) was performed for each element above the LOQ to statistically determine if the elemental concentrations varied over time. The final average of the four replicate concentrations over the 12-week study at week 0, 2, 6, and 12 are summarized in Table 2. These concentrations will be critical in the next stage of the project to develop the IGSR microparticle suspension further and to study the correlation of concentrations 
per a given standard volume with number and composition of IGSR particles. In other words, our overall goal is to provide suspension standards with known concentrations, composition, particle counts, and size. This feasibility study represents a first step towards that ultimate goal.

Table 2: Summary of the elemental concentration for the ten primers collected (replicate B; $\mathrm{n}=4$ each)

\begin{tabular}{|c|c|c|c|c|c|c|c|c|c|c|}
\hline \multirow[b]{3}{*}{ Element } & \multicolumn{10}{|c|}{ Primer Type and concentration $(\mathrm{mg} / \mathrm{L})$} \\
\hline & \multicolumn{5}{|c|}{ Lead-free } & \multicolumn{5}{|c|}{ Leaded } \\
\hline & CCX & FIO & HEV & INC & SYN & FED & REM & SAB & TUL & WIN \\
\hline $\mathbf{K}$ & - & $8.19 \pm 0.90$ & - & - & - & - & - & - & - & - \\
\hline $\mathbf{T i}$ & - & - & - & $1.2 \pm 0.1$ & - & - & - & - & - & - \\
\hline $\mathrm{Cu}$ & - & $0.27 \pm 0.02$ & - & - & - & - & - & - & - & - \\
\hline $\mathbf{Z n}$ & - & - & - & $10.4 \pm 0.9$ & - & - & - & - & - & - \\
\hline $\mathbf{S b}$ & - & - & - & - & - & $3.0 \pm 0.3$ & $1.5 \pm 0.2$ & $1.0 \pm 0.1$ & $1.7 \pm 0.3$ & $3.1 \pm 0.3$ \\
\hline $\mathbf{B a}$ & $3.5 \pm 0.5$ & - & $5.9 \pm 0.8$ & - & - & $11.8 \pm 2.1$ & $8.1 \pm 1.6$ & $3.6 \pm 0.4$ & - & $6.1 \pm 1.0$ \\
\hline $\mathbf{P b}$ & - & - & - & - & - & $7.1 \pm 1.4$ & $2.4 \pm 0.3$ & $2.5 \pm 0.4$ & $16.1 \pm 3.5$ & $5.0 \pm 0.9$ \\
\hline $\mathbf{B i}$ & - & - & $0.012 \pm 0.007$ & - & $0.6 \pm 0.1$ & - & - & - & - & - \\
\hline
\end{tabular}

ANOVA is a statistical test that groups the data by a factor of interest (in this study, the week of analysis) and represents the total variation of replicate sets based on the between and within group variance. Each result of ANOVA testing provides information about the different sources of variance that can be compared to samples of the same group and samples of different groups. The probability ( $\mathrm{p}$-value) of the replicate sets being statistically different can be determined by dividing the between group mean squares by the within group mean squares which results in the experimental F-value. If the ratio of calculated F-value to F-critical using an Fdistribution chart results in a p-value less than $0.05(\alpha)$, then the null hypothesis (no significant differences of concentrations over time) can be rejected and the groups are found significantly different at a given confidence interval. In this study, each of the tests at weeks $0,2,6$, and 12 was consider a factor, or group, and the elemental concentration was considered stable if there was no significant difference in the concentration of the IGSR suspension over time (i.e., a p-value greater than 0.05). If a significant difference is observed, then a post-hoc Tukey-Kramer test was used to determine which of the replicates varied in the collection. 
Figure 1 is an example of a replicate (cartridge B) for two primers, one of a standard ammunition (TULAMMO) and one of a lead-free ammunition (Inceptor) evaluated over the 12week stability study. The line in the center of the graphs represents the overall mean between the groups, the line in the center of the diamonds represents the individual group mean, and the vertical span represents the $95 \%$ confidence interval. The similar size of the Tukey-Kramer circles confirms comparable variance across weeks, and the overlapping circles indicate the samples belong to the same group. All the elements above LOQ showed no significant difference in the elemental concentrations over time. This analysis was performed for five leaded and five lead-free primer samples for the elemental concentrations above the LOQ and the average results for the 12week study is shown in Table 2. The elements showed 4-20\% intra-week variation for the calculated elemental concentrations and can be found in section 4: chapter 1. Table 2 displays the measurements for one GSR microparticles suspension replicate (B) for each of the collected ammunition types. The process of discharging the primer varies due to many uncontrollable

\section{TULAMMO primer-only Replicate B}
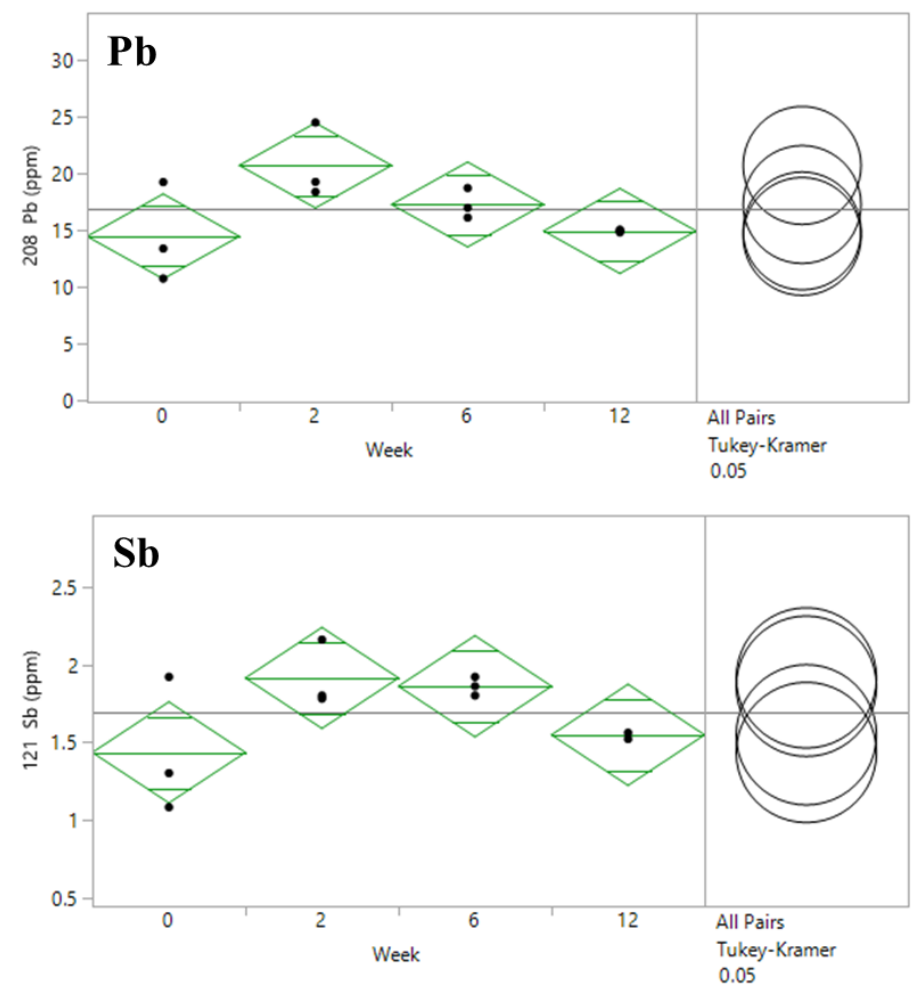

Inceptor primer-only Replicate B
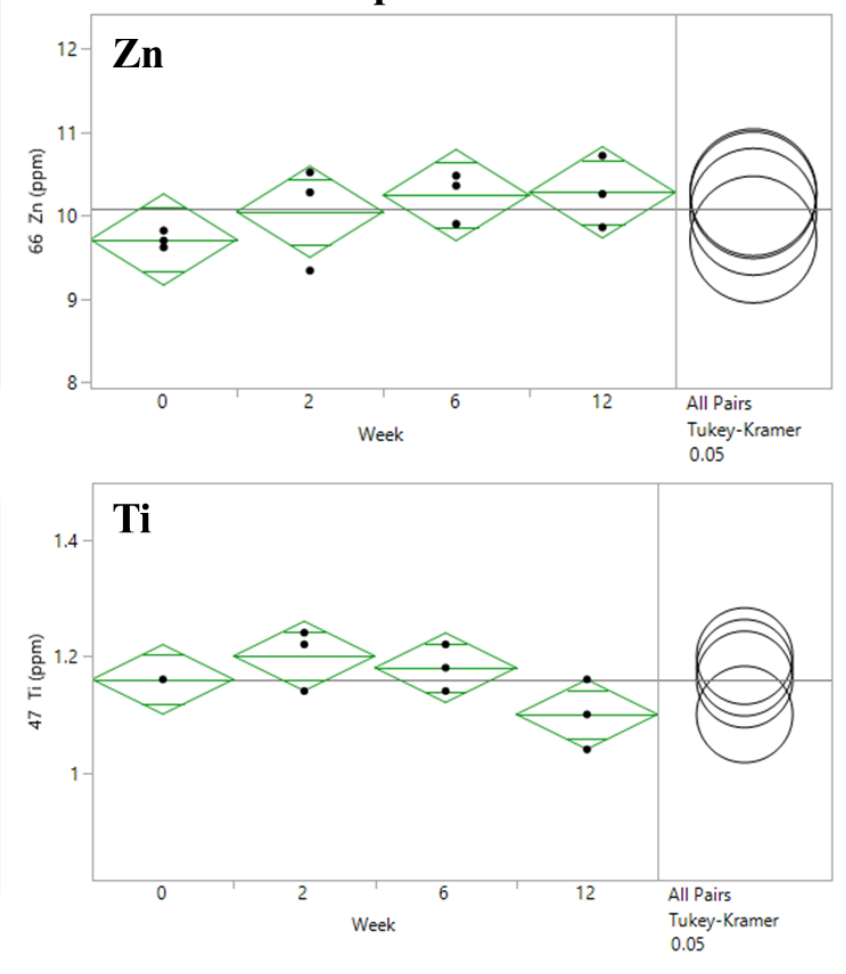

Figure 1: ANOVA and Tukey-Kramer results for two in-house primer-only samples. The results confirm the elements of interest (possible GSR) are remaining stable during the 12-week storage period. 
variables inherent to the discharge event. It is experimentally difficult, if not impossible, to exactly reproduce the number of GSR microparticles produced in a firing event, so all the replicate suspensions were treated as independent stock standards, although the overall elemental composition was consistent within each primer type.

There was only one exception to all the replicate primer collections containing consistent elemental composition and had different IGSR elements observed from the three different primer collections: Inceptor brand primer (INC) replicate C. Replicate A and B of INC contained leadfree IGSR elements of titanium and zinc, while replicate $\mathrm{C}$ had unexpected leaded elements of lead, barium and antimony, and the ANOVA and Tukey-Kramer results are statistically illustrated in figure 2. All three primers collected originated from the same box of ammunition, but the elemental composition within the same box was not uniform. Therefore, the significant difference in primer composition was attributed to a mixed composition in the manufacturer's ammunition box, which was sold as lead-free. This result is an example of unintentional mistakes during the storage of the primers, and the eventual loading and packaging into boxes for sale. Primers have a very long shelf-life when stored properly; therefore, large batches can be made and stored until ready to be loaded into ammunition, meaning manufacturer lots can be unintentionally mixed, resulting in ammunition having primers with different elemental compositions. In figure 2, the mean of replicate $\mathrm{C}$ over the 12-week study, along with the Tukey-Kramer test, confirm a significant difference between the first two pieces of ammunition (A and B) and the third one (C): the former has titanium and zinc, while the latter contains antimony, lead, and barium. Also interesting is the absence of titanium and zinc when lead, barium, and antimony are present and vice versa.

These results were further confirmed by LIBS and SEM-EDS. This situation provides an example of differences in elemental composition that can be observed between ammunition within the same box and the additional challenges to determining specific combinations of elements for a specific brand of ammunition. These results, however, do not have negative implications for the IGSR suspension standards, as every standard suspension is fully characterized and considered its own entity. Given the massive amount of IGSR particles contained in each 100mL standard ( 12 to 48 million microparticles) and small volumes $(20-100 \mathrm{uL})$ required to create a dry-form singlestandard, each $100 \mathrm{~mL}$ suspension can generate up to 5,000-1,000 individual standard samples. 

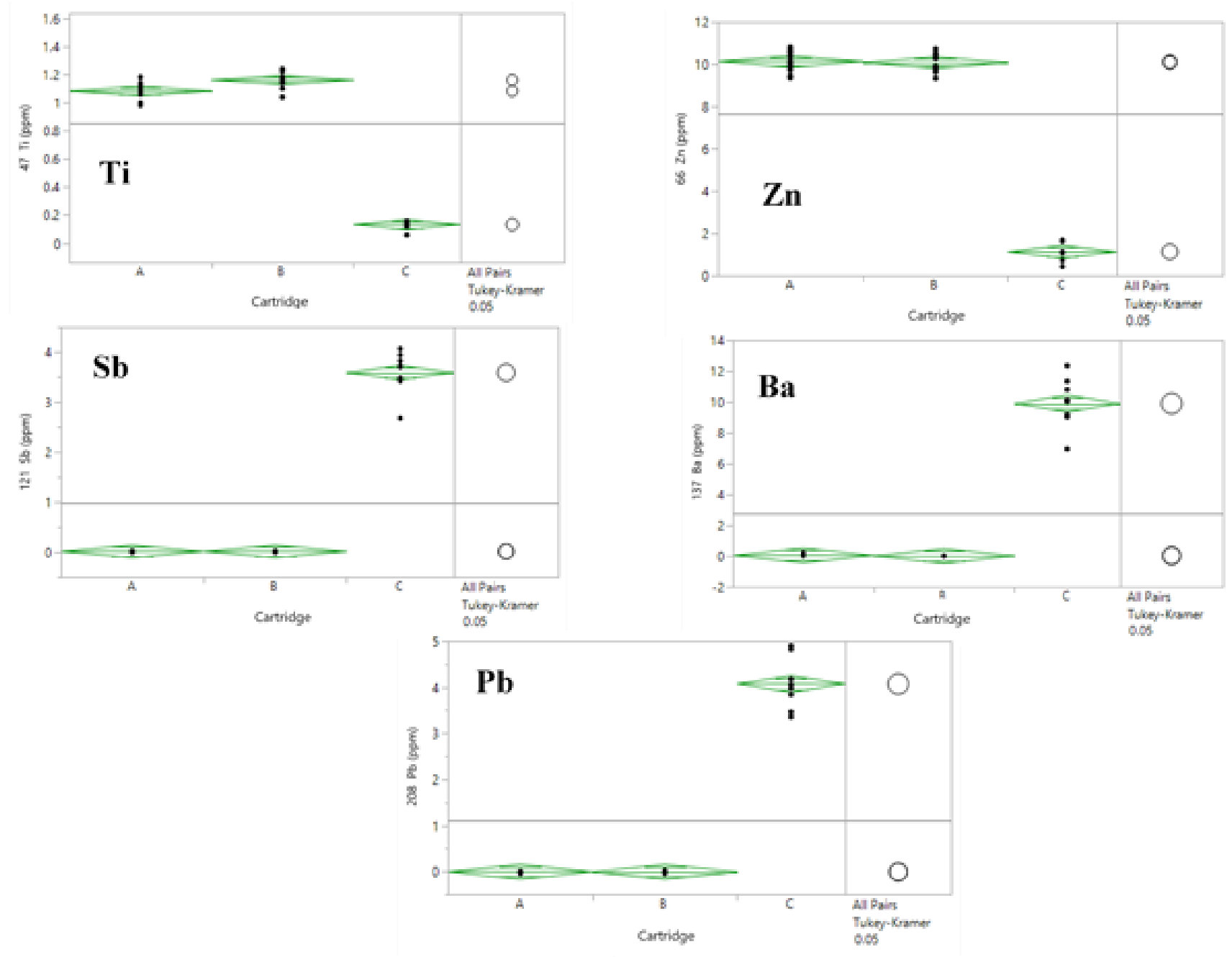

Figure 2:Inceptor primer-only data from 12-week ICP-MS study ANOVA and Tukey-Kramer results. Ti and Zn are common elements in lead-free ammunition, and $\mathrm{Sb}, \mathrm{Ba}$, and $\mathrm{Pb}$ are common elements in leaded ammunition. The replicates collected show both types of GSR elements. 
Overall, it was found that lead-free primers have a more variable elemental composition, while the leaded ammunition has relatively less variable elemental signatures at different concentrations, as can be seen in Table 2. SYNTECH (SYN) ammunition only contains bismuth, while Fiocchi (FIO) has potassium with a low concentration of copper. In the case of INC, only titanium and zinc were detected in two replicates, while only lead, barium, and antimony were detected in the third replicate, as previously discussed. Federal (FED), Remington (REM), Sellier and Bellot (SAB), and Winchester (WIN) are all leaded ammunition types and contain the typical GSR elements: antimony, lead, and barium. However, TULAMMO (TUL) is an interesting leaded ammunition because it lacks barium, which is an important element in the identification of GSR. Under the current ASTM standard practice E1588, the lack of barium in the TUL particles would direct to the classification of the particles as "consistent with GSR" since the particle only contains lead and antimony. A higher degree of confidence to be classified as "characteristic of GSR" would require the presence of $\mathrm{Ba}$ in addition to $\mathrm{Pb}$ and $\mathrm{Sb}$.

These preliminary results highlight the relevance of characterizing IGSR primers to provide better tools for understanding and evaluating IGSR, as the composition of modern ammunition is relatively variable. Despite the inherent between-cartridge variation for the number of IGSR particles collected, the stability observed on a single cartridge/primer standard suspension serves as proof of principle that the proposed method can be used to create leaded and lead-free IGSR reference standards for quality control purposes. Moreover, the typical volume of the standard required for analysis (typically $20-100 \mu \mathrm{L}$ in this study) would allow a single primer standard to prepare thousands of IGSR reference standard stubs that could be further diluted depending on the sensitivity of technique being tested. Therefore, each standard suspension has the potential to be used in future method validations, as daily quality control, and for interlaboratory studies.

\subsubsection{SEM-EDS Analysis}

Critical components of the characterization of the IGSR microparticle standards are to confirm the elemental composition, monitor the integrity of the morphology during prolonged storage, and estimate the position and count of the particles deposited for a given volume. Ideally, the reference standards could be stored in a laboratory for a prolonged period of time and the suspensions could be accessed as needed. SEM-EDS analysis is ideal for detecting elemental 
composition, monitoring the morphology, and mapping the homogeneity of the deposited microparticle suspensions. The results provided information about the elemental composition, morphology, and deposition of the particles selected by the software and manually confirmed by the researcher.

When analyzing the GSR microparticles samples, the SEM-EDS data was reviewed by the analyst to evaluate the elemental composition and the morphology of the GSR microparticles. An important part of GSR analysis is the confirmation of the elemental composition within a micrometer-sized single particle. The morphology is important because of how GSR is created during the firing process. When a firearm is discharged, the ammunition undergoes a rapid, high temperature and pressure thermodynamic process as it expels the bullet from the barrel. The plume of the created GSR is subjected to rapid heating and cooling, causing the molten components to condense and form spheroid particles. This process of particle formation can be explained by the cohesive forces applied as the particle cools. ${ }^{4}$ While particles irregular in shape are also observed in IGSR, identifying specific elemental combinations within a particle greatly increases the confidence in GSR identification.

The IGSR microparticle suspension standards produced the spheroid microparticles expected when analyzing IGSR. Figure 3 provides examples of GSR particles from leaded ammunition with their typical shape and size. Winchester is a typical leaded primer that contains all three characteristic GSR elements of lead, barium, and antimony. TULAMMO is an interesting example of a leaded primer because the particles in this standard ammunition lack barium as part of the elemental composition, which was corroborated by ICP-MS and LIBS.

On the other hand, Figure 4 provides examples of lead-free primers with an irregular surface morphology and elements that are not distinctive of GSR. Some compositions from leadfree traces are more difficult to distinguish from non-GSR environmental particles. An item of evidence using Fiocchi or SYNTECH ammunition (e.g., K, Si, $\mathrm{Cu}$, and $\mathrm{Al}$ or $\mathrm{Bi}$ and $\mathrm{Al}$ particle respectively) would not be identified as characteristic or consistent with GSR based on the current ASTM criterion, demonstrating the relevance of this study in increasing the knowledge of morphology and chemical composition of diverse modern ammunition and providing an expanded variety of IGSR standards.

Moreover, the occurrence of lead-free ammunition residues presents instrumental challenges to the forensic scientist as the traces are often lower atomic number elements that lack the bright 
contrast of heavy metals under the backscatter detector, making it more difficult to distinguish from the background during the automated particle discovery phase. Indeed, alternative detectors such as cathodoluminescence and Raman have been recently suggested to aid SEM-EDS examination of atypical elemental compositions. ${ }^{45,46}$ The availability of heavy metal-free standards would be useful for the optimization of automated analysis by SEM-EDS.

The deposition of the IGSR microparticle suspension was tested using the dried, diluted form of the IGSR microparticle suspensions and placing the dried samples directly in the SEM-EDS sample chamber to map the areas containing microparticles using the automated software. After analysis, the features detected were manually examined to determine if the microparticles spread homogenously within the deposited area. Figure 5 shows the map of particles generated by the deposition of the IGSR microparticle for a TULAMMO primer suspension. The edges of the spot are well-defined, and the particles are dispersed relatively evenly throughout the area. The reproducible distribution of the IGSR microparticle suspension in a finite area shows the potential of the reference standards for future research on transfer and persistence of GSR evidence, where it is essential to know both the elemental composition along with the ground truth number of microparticles. The microparticle suspensions also vary in size $(1-10 \mu \mathrm{m})$ as can be seen in Figure 5 , and this provides a variety of microparticles to analyze. In general, approximately 120-500 particles were present per microliter of the stock suspension, depending on the primer type. These finding show the standards are viable as quality assurance controls for SEM-EDS and for interlaboratory testing. Ongoing interlaboratory studies in our research group aim to further evaluate the reproducibility of particle count and size that will allow a more comprehensive characterization of the materials to be used as laboratory reference samples.

The results from the particles identified by SEM-EDS analysis are also in agreement with the elemental profiles observed by ICP-MS, providing a greater level of confidence in the conclusions for the different primer standards. While ICP-MS cannot provide morphological information, the elemental composition can be determined with high sensitivity, reproducibility, and accuracy, making it ideal for validation, cross-validation, and interlaboratory studies. The use of complementary methods is important when analyzing the elemental composition of modern ammunition and to further expand the understanding of reported IGSR composition in the literature. Also, the multi-technique cross-validation performed in this study provides a basis for future applications of these laboratory standards for quality control and method development in a 


\section{Winchester}

\section{Typical leaded primer}

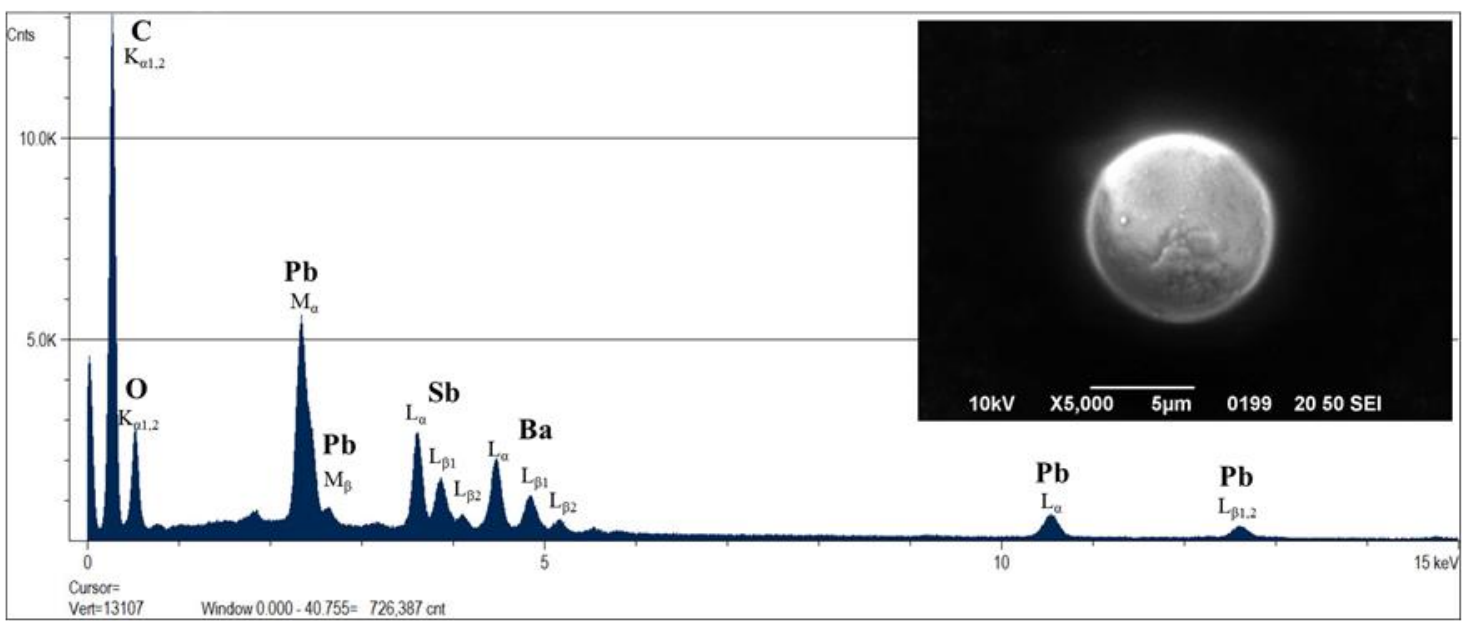

\section{TULAMMO}

Leaded primer without $\mathrm{Ba}$

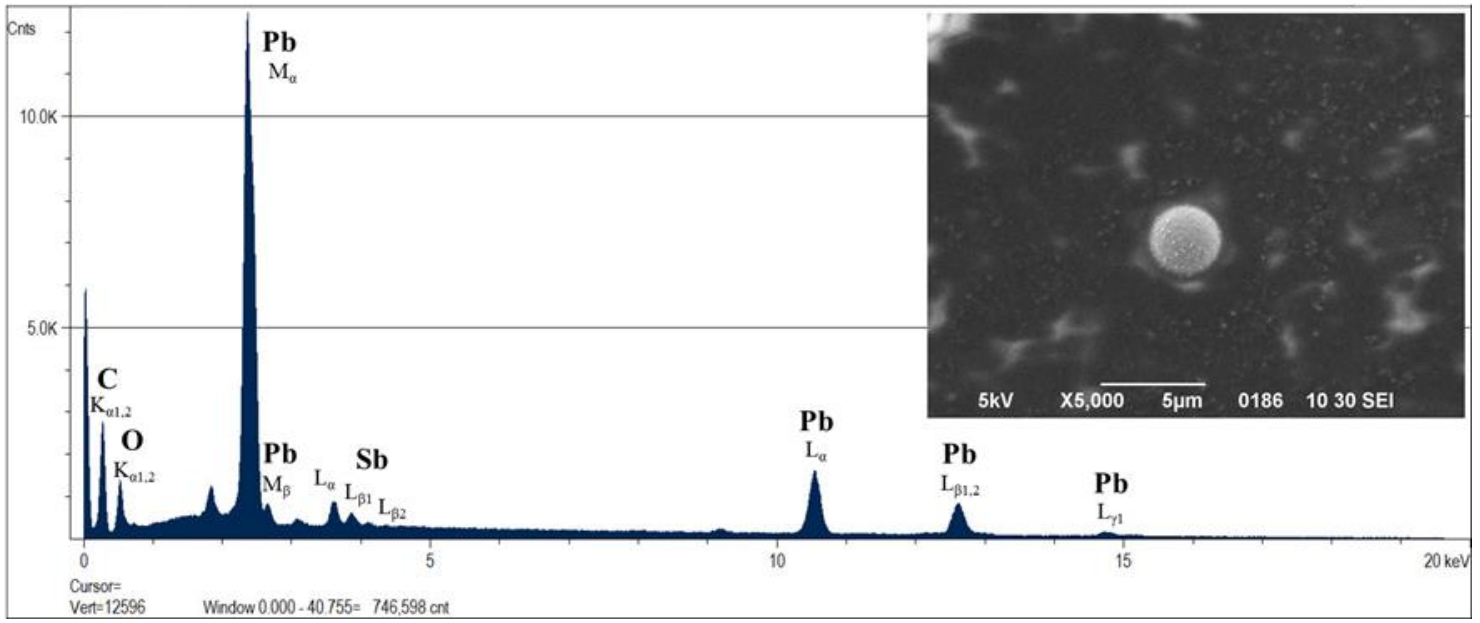

Figure 3: Examples of microparticles after 12-weeks of stability. Top is a particle generated by Winchester: standard, leaded primer with antimony, barium, and lead. Bottom is a particle generated by TULAMMO: standard, leaded primer that lacks barium. The imbedded images were taken using SEM and spectrum was generated using EDS 


\section{Fiocchi}

\section{Lead-free primer with a light elements}

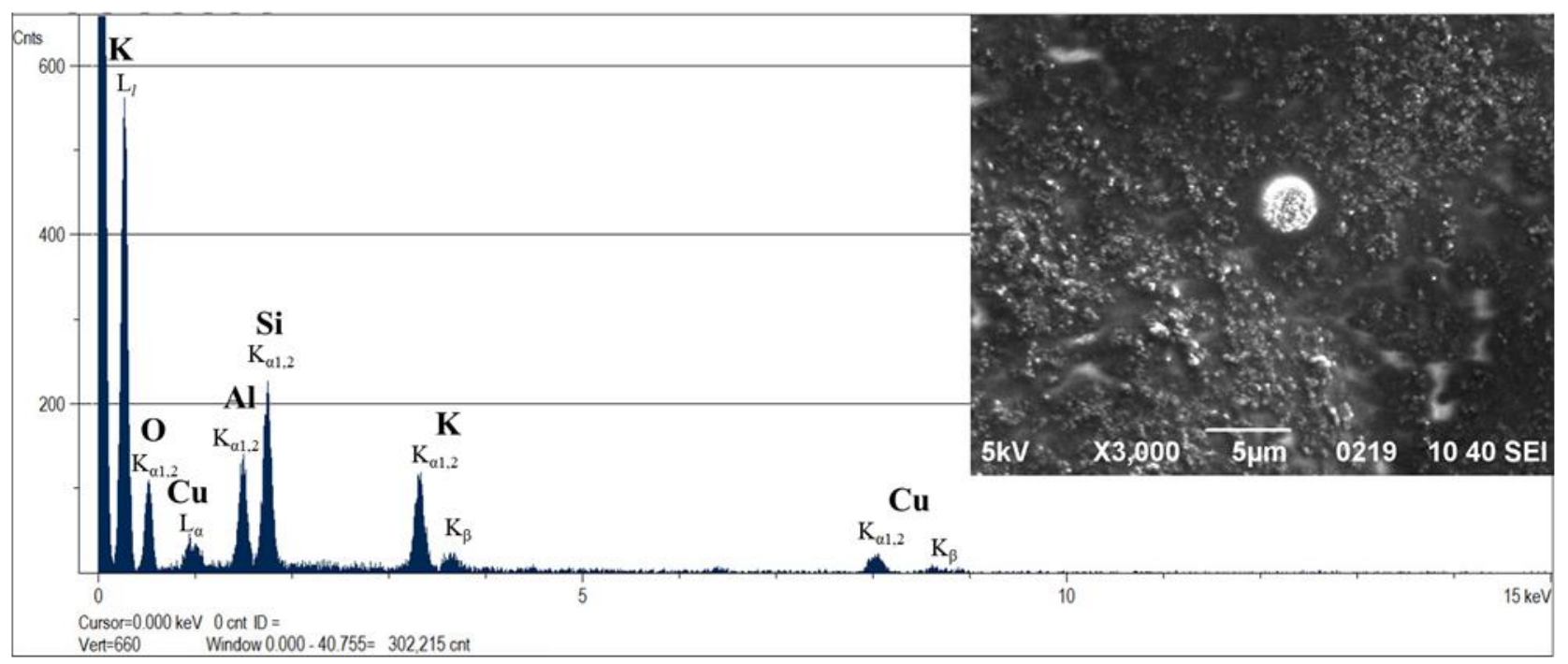

\section{SYNTECH}

\section{Lead-free primer with a heavy element}

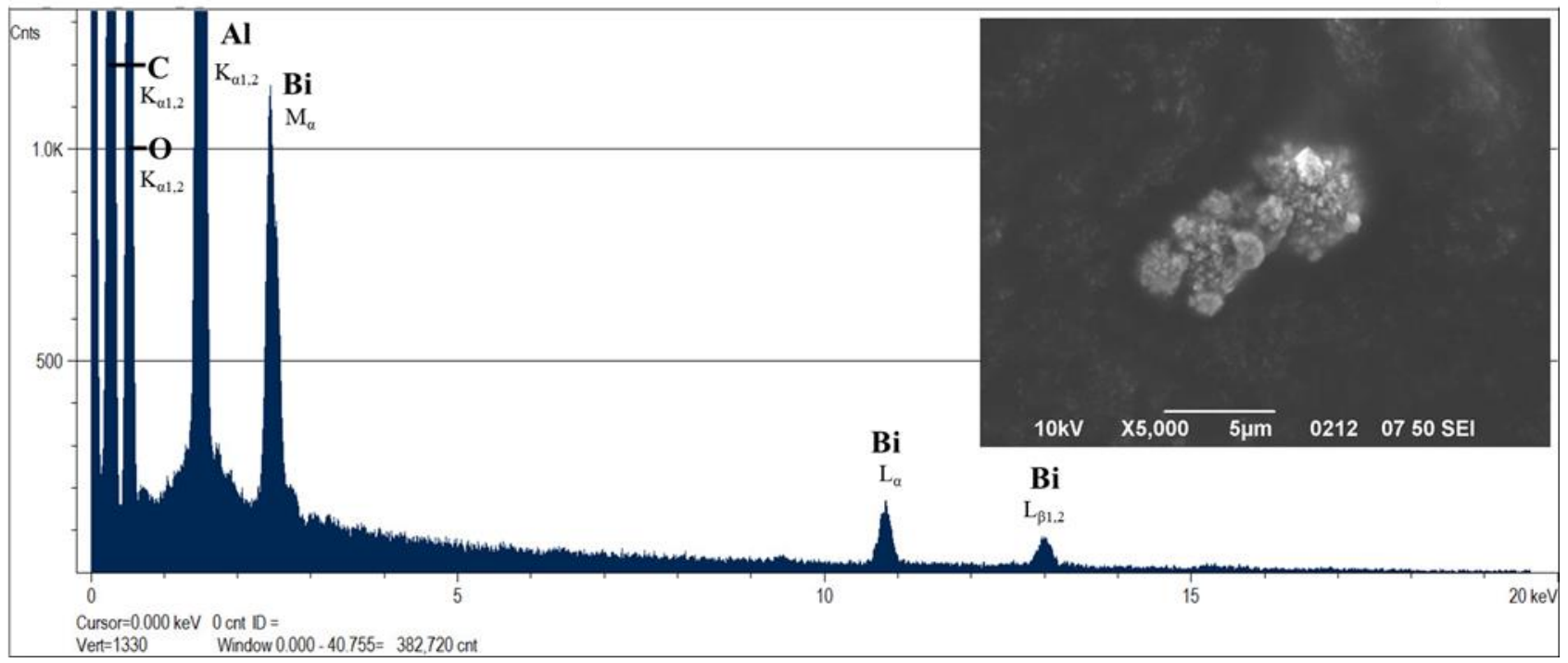

Figure 4: Examples of microparticles after 12-weeks of stability. Top is a particle generated by Fiocchi: lead-free primer with only a light atomic weight element as a GSR elements. Bottom is a particle generated by SYNTECH: lead-free primer with only one heavy atomic weight element as a GSR element. Both microparticles have common background elements (aluminum in both and silicon-Fiocchi only) incorporated into the particle. The imbedded images were taken using SEM and spectrum was generated using EDS. 


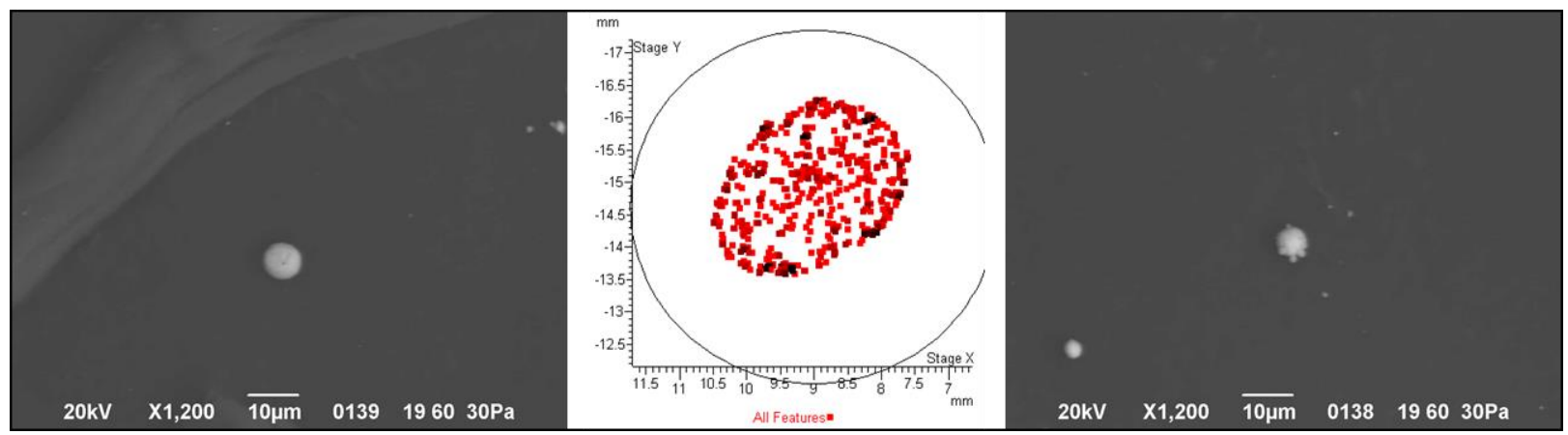

Figure 5: Example of SEM-EDS particle mapping for a $2 \mu \mathrm{L}$ deposit of a TULAMMO microparticle reference suspension containing a total of 325 particles and SEM images of the varying sizes of particles in the microparticle reference suspension.

variety of fields. The capability to monitor residues in microparticle form is particularly useful in studies where knowledge of persistence over time are critical, such as forensic, environmental, or occupational safety analyses.

\subsubsection{LIBS Analysis}

The IGSR microparticle suspensions collected showed stability in elemental concentration and morphology over time. After completing ICP-MS and SEM-EDS analysis on the samples, they were applied to the development and validation of two LIBS methods, as reported in MenkingHoggatt et. al.. ${ }^{57}$ The IGSR microparticle suspensions were applied to optimize and compare different ablation patterns and settings. Some of the suspensions were also used as daily quality controls and instrument performance tests for the developed method. This was possible because the microparticles were evenly distributed across the carbon stub allowing the use of the standards to build quality control charts over several months. The application of the IGSR microparticle suspensions demonstrated the utility of these reference suspensions for the development, optimization, and validation of new, rapid LIBS method for the detection of IGSR and for quality control and daily performance. These tailored standards have also been applied in our research group for the development and validation of Liquid Chromatography-Mass Spectrometry (LCMS) and electrochemical methods for IGSR detection. Ongoing research is also making use of these standards for the study of transfer and persistence mechanisms, demonstrating their versatility and applicability in the field. 
LIBS also provided a third source of complementary information about the elemental composition of the IGSR microparticles suspensions. Table 3 provides a summary of all three techniques and the elements identified above the LOD. Analysis by LIBS confirmed the presence of elements detected by the other techniques, with the advantage that LIBS is much faster $(\sim 1$ $\mathrm{min} / \mathrm{sample}$ by LIBS vs. $2-8 \mathrm{~h} / \mathrm{sample}$ by SEM-EDS, depending on the sample and instrumental setup), does not require any sample preparation, and analysis can be conducted directly on carbon

SEM stubs with over $99 \%$ of the stub remaining unaltered after analysis. ${ }^{57}$ Also, we have reported in previous studies the ability of LIBS to provide spatial information along with simultaneous detection of multiple elements (e.g., $\mathrm{Pb}, \mathrm{Ba}$, and $\mathrm{Sb}$; $\mathrm{Ti} / \mathrm{Zn}$; $\mathrm{Bi}$; etc.) from the same location $(\sim 100 \mu \mathrm{m}) .{ }^{57}$ This sampling approach increases the confidence in the results that the elements come from a particle or group of particles, located in a small area rather than somewhere else within the trace. This spatial information does not match the ideal single-particle information provided by SEM-EDS but provides added value over bulk analysis, mainly when the complementary nature to SEM-EDS is considered in specific case circumstances (e.g., challenging lead-free or heavy metal-free ammunition, rapid screening in multiple items cases). The spatial information that LIBS does provide, along with the limited sample destruction, is another advantage of LIBS as a screening method complementary to SEM-EDS.

Table 3: Summary of the elements detected in the IGSR microparticle suspensions separated by all techniques and primer type.

\begin{tabular}{cccccc}
\hline $\begin{array}{c}\text { Primer Brand } \\
\text { Name }\end{array}$ & $\begin{array}{c}\text { Primer } \\
\text { Abbreviation }\end{array}$ & $\begin{array}{c}\text { Lead- } \\
\text { free }\end{array}$ & ICP-MS & SEM-EDS & LIBS \\
\hline CCI- non-leaded & $\mathrm{CCX}$ & yes & $\mathrm{Ba}$ & $\mathrm{Ba}$ & $\mathrm{Ba}$ \\
Fiocchi & $\mathrm{FIO}$ & yes & $\mathrm{K}, \mathrm{Cu}$ & $\mathrm{K}, \mathrm{Cu}$ & $\mathrm{K}, \mathrm{Cu}$ \\
Hevi-shot & $\mathrm{HEV}$ & yes & $\mathrm{Ba}$ & $\mathrm{Ba}$ & $\mathrm{Ba}$ \\
Inceptor RNPTM & $\mathrm{INC}$ & yes & $\mathrm{Ti}, \mathrm{Zn}$ & $\mathrm{Ti}, \mathrm{Zn}$ & $\mathrm{Ti}$ \\
SYNTECH federal & $\mathrm{SYN}$ & yes & $\mathrm{Bi}$ & $\mathrm{Bi}$ & - \\
Federal & $\mathrm{FED}$ & no & $\mathrm{Sb}, \mathrm{Pb}, \mathrm{Ba}$ & $\mathrm{Sb}, \mathrm{Pb}, \mathrm{Ba}$ & $\mathrm{Sb}, \mathrm{Pb}, \mathrm{Ba}$ \\
Remington & REM & no & $\mathrm{Sb}, \mathrm{Pb}, \mathrm{Ba}$ & $\mathrm{Sb}, \mathrm{Pb}, \mathrm{Ba}$ & $\mathrm{Sb}, \mathrm{Pb}, \mathrm{Ba}$ \\
Sellier \& Bellot & $\mathrm{SAB}$ & no & $\mathrm{Sb}, \mathrm{Pb}, \mathrm{Ba}$ & $\mathrm{Sb}, \mathrm{Pb}, \mathrm{Ba}$ & $\mathrm{Sb}, \mathrm{Pb}, \mathrm{Ba}$ \\
TulAmmo & TUL & no & $\mathrm{Sb}, \mathrm{Pb}$ & $\mathrm{Sb}, \mathrm{Pb}$ & $\mathrm{Sb}, \mathrm{Pb}$ \\
Winchester & WIN & no & $\mathrm{Sb}, \mathrm{Pb}, \mathrm{Ba}$ & $\mathrm{Sb}, \mathrm{Pb}, \mathrm{Ba}$ & $\mathrm{Sb}, \mathrm{Pb}, \mathrm{Ba}$ \\
\hline
\end{tabular}

The examination of SYN ammunition proved to be challenging due to the low concentration of Bi present in the suspension $(\sim 0.7 \mathrm{mg} / \mathrm{L})$, which was below LIBS limits of 
detection for this element. When the samples were analyzed using a sensitive Intensified Charge Coupled Device (ICCD) detector, Bi was clearly detected in the sample (figure 6). Therefore, the IGSR suspensions provided a way to test the instrument capabilities and can be used as a tool to monitor instrument performance and validate new methodologies for the detection of inorganic gunshot residues.

Finally, although LIBS is not used routinely in crime laboratories, several researchers have demonstrated its potential utility in the field. ${ }^{7,37,38,49-57}$ For example, one advantage of LIBS is its capability to assist end-users to make quick, informed decisions at the scene and laboratory that can lead to more effective case management. LIBS has shown to provide a fast and effective tool for IGSR detection, on the hands of shooters and other surfaces. The IGSR suspension standards presented in this research study provided our earlier research with a consumable IGSR material that gave us the ability to optimize a method for the screening of IGSR on the hands of shooters and an additional sample for daily monitoring of instrument performance that was similar to the hand samples being analyzed. A suspension of IGSR is not only applicable to forensic methods but versatile enough to be broadly applied to other disciplines, such as exposure and environmental studies of GSR residues in the air, soil, and other surfaces.

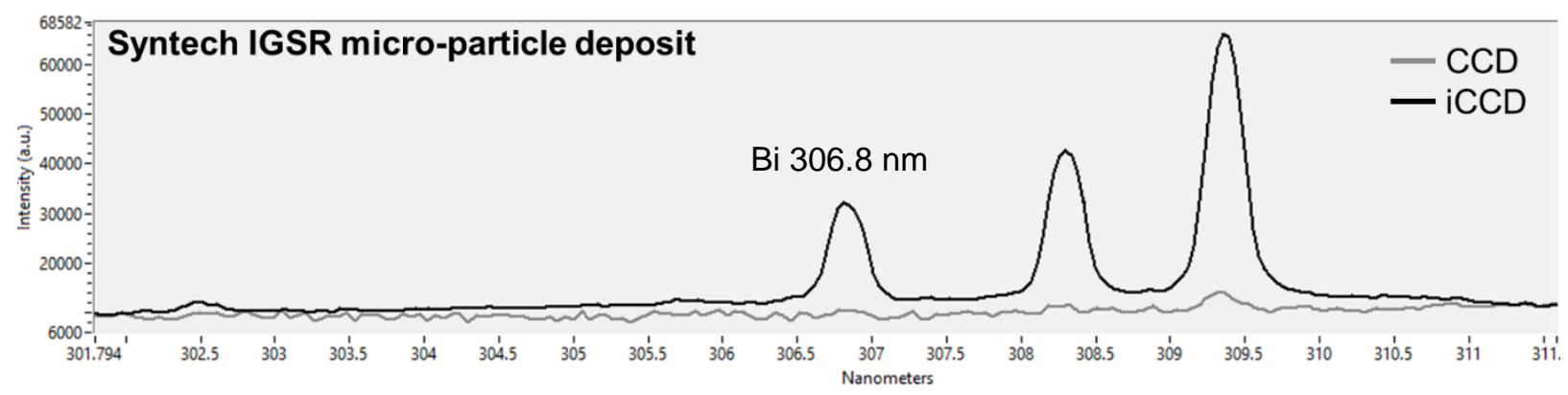

Figure 6: Spectral comparison of Syntech ammunition and the detection sensitivity of Bi with two different types of detectors and the same J200 LIBS configuration.

\subsubsection{Comparison of the Microparticle Standards to IGSR Collected from Known Shooter's Hands}

One of the purposes of the microparticle standards was to create IGSR-like standard materials of varied composition that can be used primarily for quality control purposes and for research. Although discharging a cartridge where the propellant and projectile have been removed provides a different thermal environment to a normal discharge, it was demonstrated the IGSR 
particles of the tailor-made standards were very similar in composition and morphology. To illustrate this, Figure 7 and Table 4 shows similar compositions observed for some primer-only standards as compared to the samples collected from the shooter's hands using full ammunition. Figure 7 illustrates the comparable morphology and elemental composition from a leaded WIN ammunition and a lead-free FIO particle collected from the hands of a shooter. Table 4 provides the primary elemental composition observed in the samples, although some additional commonly associated elements were randomly observed, such as aluminum, titanium, zinc, copper, and silicon. Detection of these additional elements were variable in both types of samples analyzed.

As shown in the Winchester example in Figures 3 and 7, the relative ratios of lead, barium, and antimony are not identical in all the IGSR particles. However, rather than creating "identical" composition, morphology, and distribution of particles for each case/ammunition, the intended use of these tailor-made materials is to provide a variety of matrix-matched standards resembling typical IGSR and expanded compositions representative of leaded and lead-free ammunition.

It is important to clarify that the tailor-made standards developed in this study are intended to be used as characterized control materials rather than "known case reference" samples. In some cases, examiners can compare the IGSR composition from hands of an individual of interest to those residues of related items such as spent cartridges or clothing. In such circumstances, those compared items are used as "known" primer reference samples to aid in the interpretation of the evidence. There are various ways in which the laboratories prepare reference samples, and a full characterization of those standards, like the multi-method approach proposed here, is not needed. Likewise, a composition and distribution of particles "identical" to a fired loaded ammunition is not needed for developing the microparticle standards, because the aim is to produce "similar" IGSR particles with known features and composition, which can be reproducibly used as quality controls. Therefore, the comparison of primer-only and full ammunition IGSR was conducted as a proof of principle that the microparticles resemble features that will be valuable for standard materials in quality assurance, and fundamental or application research settings. 


\section{Winchester GSR particle from the hand of a known-shooter}

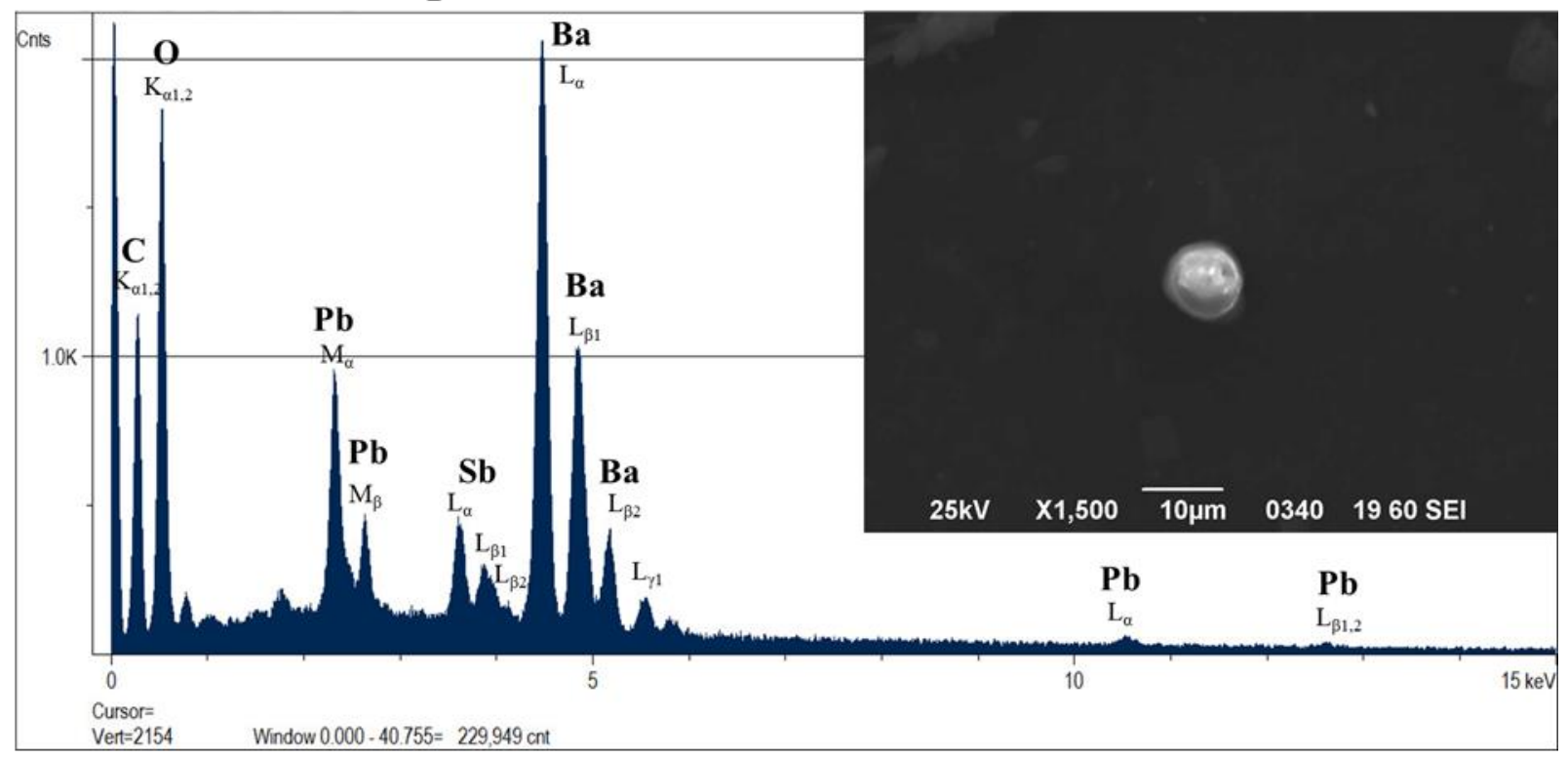

Fiocchi GSR particle from the hand of a known-shooter

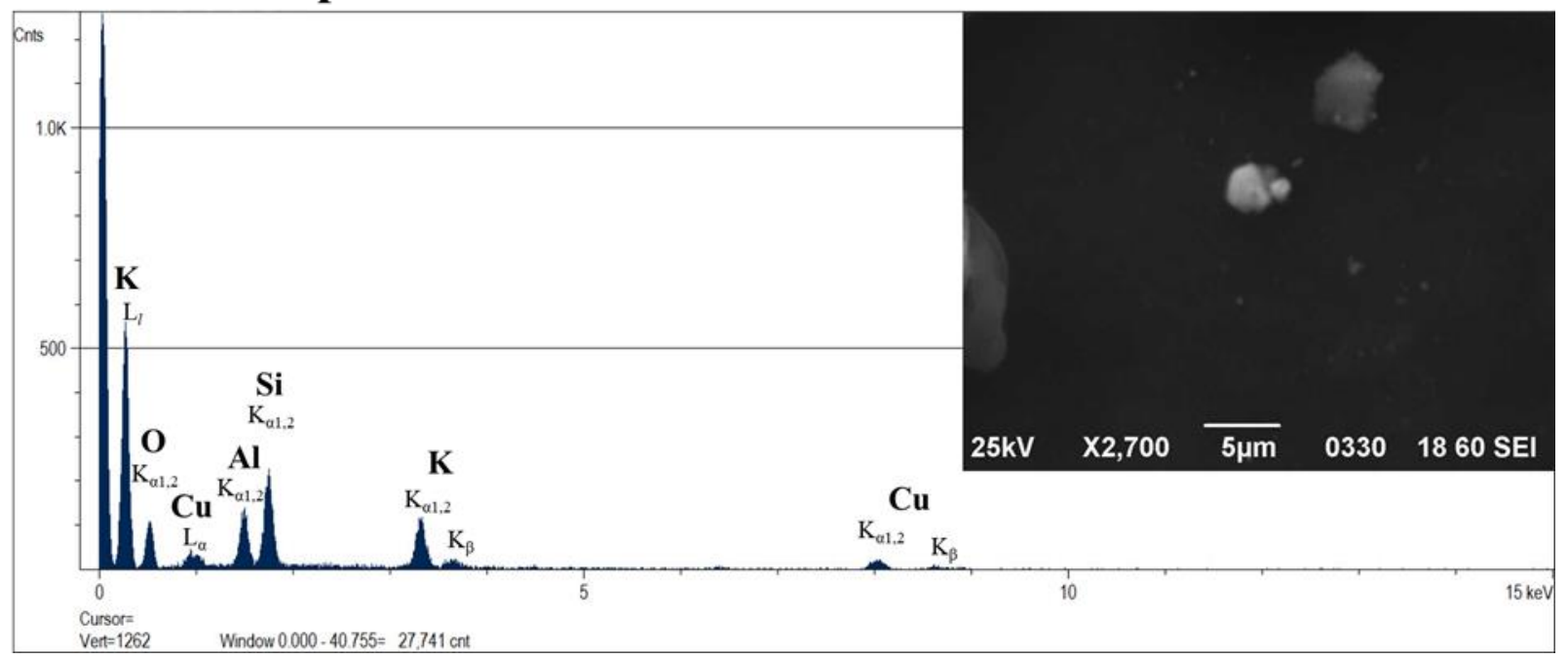

Figure 7: GSR particles collected from the hands of known-shooters with ammunition using the same brand and lot of primers as the IGSR microparticle standards created. The IGSR microparticles collected with only a primer and a cartridge case exhibit the sample elemental composition and morphology as known-shooter samples collected from the hands. Winchester is a leaded ammunition and Fiocchi is a lead-free ammunition. 
Table 4: Summary of the elements and morphology detected in the IGSR microparticle suspensions separated by all techniques and primer type.

\begin{tabular}{|c|c|c|c|c|c|c|}
\hline \multirow[b]{2}{*}{$\begin{array}{c}\text { Primer } \\
\text { Brand Name }\end{array}$} & \multirow[b]{2}{*}{$\begin{array}{c}\text { Primer } \\
\text { Abbreviation }\end{array}$} & \multirow[b]{2}{*}{$\begin{array}{l}\text { Lead- } \\
\text { free }\end{array}$} & \multicolumn{2}{|c|}{ IGSR microparticle standard } & \multicolumn{2}{|c|}{ Known-shooter hand samples } \\
\hline & & & $\begin{array}{c}\text { Primary } \\
\text { elemental } \\
\text { composition }\end{array}$ & Morphology & $\begin{array}{c}\text { Primary } \\
\text { elemental } \\
\text { composition }\end{array}$ & Morphology \\
\hline $\begin{array}{l}\text { CCI- non- } \\
\text { leaded }\end{array}$ & $\mathrm{CCX}$ & yes & $\mathrm{Ba}$ & $\begin{array}{l}\text { Spheroid and } \\
\text { irregular }\end{array}$ & $\mathrm{Ba}$ & $\begin{array}{l}\text { Spheroid and } \\
\text { irregular }\end{array}$ \\
\hline Fiocchi & FIO & yes & $\mathrm{K}, \mathrm{Cu}$ & $\begin{array}{l}\text { Spheroid and } \\
\text { irregular }\end{array}$ & $\mathrm{K}, \mathrm{Cu}$ & $\begin{array}{l}\text { Spheroid and } \\
\text { irregular }\end{array}$ \\
\hline Hevi-shot & HEV & yes & $\mathrm{Ba}$ & $\begin{array}{l}\text { Spheroid and } \\
\text { irregular }\end{array}$ & $\mathrm{Ba}$ & $\begin{array}{l}\text { Spheroid and } \\
\text { irregular }\end{array}$ \\
\hline $\begin{array}{l}\text { Inceptor } \\
\text { RNPTM }\end{array}$ & INC & yes & $\mathrm{Ti}, \mathrm{Zn}$ & $\begin{array}{l}\text { Spheroid and } \\
\text { irregular }\end{array}$ & $\mathrm{Ti}, \mathrm{Zn}$ & $\begin{array}{l}\text { Spheroid and } \\
\text { irregular }\end{array}$ \\
\hline $\begin{array}{c}\text { SYNTECH } \\
\text { federal }\end{array}$ & SYN & yes & $\mathrm{Bi}$ & $\begin{array}{l}\text { Spheroid and } \\
\text { irregular }\end{array}$ & $\mathrm{Bi}$ & $\begin{array}{l}\text { Spheroid and } \\
\text { irregular }\end{array}$ \\
\hline Federal & FED & no & $\mathrm{Sb}, \mathrm{Pb}, \mathrm{Ba}$ & $\begin{array}{l}\text { Spheroid and } \\
\text { irregular }\end{array}$ & $\mathrm{Sb}, \mathrm{Pb}, \mathrm{Ba}$ & $\begin{array}{l}\text { Spheroid and } \\
\text { irregular }\end{array}$ \\
\hline Remington & REM & no & $\mathrm{Sb}, \mathrm{Pb}, \mathrm{Ba}$ & $\begin{array}{l}\text { Spheroid and } \\
\text { irregular }\end{array}$ & $\mathrm{Sb}, \mathrm{Pb}, \mathrm{Ba}$ & $\begin{array}{l}\text { Spheroid and } \\
\text { irregular }\end{array}$ \\
\hline $\begin{array}{c}\text { Sellier \& } \\
\text { Bellot }\end{array}$ & SAB & no & $\mathrm{Sb}, \mathrm{Pb}, \mathrm{Ba}$ & $\begin{array}{l}\text { Spheroid and } \\
\text { irregular }\end{array}$ & $\mathrm{Sb}, \mathrm{Pb}, \mathrm{Ba}$ & $\begin{array}{l}\text { Spheroid and } \\
\text { irregular }\end{array}$ \\
\hline Winchester & WIN & no & $\mathrm{Sb}, \mathrm{Pb}, \mathrm{Ba}$ & $\begin{array}{l}\text { Spheroid and } \\
\text { irregular }\end{array}$ & $\mathrm{Sb}, \mathrm{Pb}, \mathrm{Ba}$ & $\begin{array}{l}\text { Spheroid and } \\
\text { irregular }\end{array}$ \\
\hline
\end{tabular}

\subsection{Conclusion}

IGSR microparticles that originated from modern leaded and lead-free primers were collected using a novel method that suspended the IGSR microparticles in an organic medium and assisted in expanding our current knowledge of modern ammunition and its elemental composition. The collected suspensions of microparticles showed excellent stability after being stored for prolonged periods of time and maintained the elemental composition and morphological integrity. The diameter of the collected microparticles, when analyzed by SEM-EDS, ranged from $0.5 \mu \mathrm{m}$ to $10 \mu \mathrm{m}$, and contained spheroid particles, demonstrating its applicability for SEM-EDS and other methods. Further analysis by different instrumentation is feasible on the collected standards because the suspensions remain stable over time, and each suspension $(\sim 100 \mathrm{~mL})$ can prepare approximately 1,000-5,000 IGSR reference stubs or spikes of 20-100uL, depending on the application and detection method. The stock standard can produce approximately 120 to 500 particles per microliter, depending on the primer type. The ability of the IGSR microparticle standards to be used in suspension or dry form allows for a multitude of uses that otherwise would be unavailable to the field.

Multiple validated analytical techniques (ICP-MS, SEM-EDS, and LIBS) confirmed the elemental composition of the IGSR microparticle suspensions and demonstrated the versatility and utility of these reference standards for research and future applications at forensic laboratories. Access to characterized reference standards would serve as a ground truth material for GSR 
research, development, and as a valuable quality control tool in routine laboratory analysis. In addition, the use of the reference suspensions could expand the detection of IGSR to further research into emerging techniques for analysis and provide a reference material for crossvalidation and interlaboratory studies. A suspension such as the ones tested in this study could provide the field of IGSR analysis access to a consumable standard, for the first time, and help to address a critical research need in GSR analysis. ${ }^{2}$

The future paths of this study are anticipated to assist many areas of IGSR analysis, including forensic and environmental applications, and provide access to a deeper study into the standardization of reference materials. The collected samples will continue to be monitored in our group to test stability over longer times. Further work also includes evaluating the uncertainty of the number of particles deposited in a given volume of suspension and testing of different storage containers. Once particle number can be estimated, this will open the door to research on the transfer and persistence of IGSR. Ongoing interlaboratory collaborations are addressing this second stage of the study. Other analytical techniques will be tested for application as a reference standard, to evaluate instrumental performance, and to validate other methodologies for IGSR detection.

\subsection{References}

(1) Standard Guide for Gunshot Residue Analysis by Scanning Electron Microscopy / Energy Dispersive X-Ray Spectrometry 1; West Conshohocken, PA, 2009.

https://doi.org/10.1520/E1588-20.2.

(2) OSAC Research Needs Assessment Form: Development of Characterized Reference Stubs https://www.nist.gov/system/files/documents/2016/12/06/rd_needs-_gsr_development_of_characterized_reference_stubs.pdf.

(3) Brozek-Mucha, Z. Variation of the Chemical Contents and Morphology of Gunshot Residue in the Surroundings of the Shooting Pistol as a Potential Contribution to a Shooting Incidence Reconstruction. Forensic Sci. Int. 2011, 210 (1-3), 31-41. https://doi.org/10.1016/j.forsciint.2011.01.031.

(4) Brozek-Mucha, Z. Distribution and Properties of Gunshot Residue Originating from a Luger 9 Mm Ammunition in the Vicinity of the Shooting Gun. Forensic Sci. Int. 2009, 183 (1-3), 33-44. https://doi.org/10.1016/j.forsciint.2008.10.010. 
(5) Kara, I.; Sarikavak, Y.; Lisesivdin, S. B.; Kasap, M. Evaluation of Morphological and Chemical Differences of Gunshot Residues in Different Ammunitions Using SEM/EDS Technique. Environ. Forensics 2016, 17 (1), 68-79.

https://doi.org/10.1080/15275922.2015.1133729.

(6) Hogg, S. R.; Hunter, B. C.; Smith, R. W. Elemental Characterization and Discrimination of Nontoxic Ammunition Using Scanning Electron Microscopy with Energy Dispersive X-Ray Analysis and Principal Components Analysis. J. Forensic Sci. 2016, 61 (1), 35-42. https://doi.org/10.1111/1556-4029.12881.

(7) Fambro, L. A.; Miller, E. T.; Vanderbos, D. D. Characterization of Lead-Free Gunshot Residue Analogs. Anal. Methods 2016, 8, 3132-3139. https://doi.org/10.1039/c6ay00725b.

(8) Fambro, L. A.; Vandenbos, D. D.; Rosenberg, M. B.; Dockery, C. R. Laser-Induced Breakdown Spectroscopy for the Rapid Characterization of Lead-Free Gunshot Residues. Appl. Spectrosc. 2017, 71 (4), 699-708. https://doi.org/10.1177/0003702816689099.

(9) Gunaratnam, L.; Himberg, K. The Identification of Gunshot Residue Particles from LeadFree Sintox Ammunition. J. Forensic Sci. 1994, 39 (2), 532-536. https://doi.org/10.1520/jfs13626j.

(10) Charpentier, B.; Desrochers, C. Analysis of Primer Residue from Lead Free Ammunition by X-Ray Microfluorescence. J. Forensic Sci. 2000, 45 (2), 447-452. https://doi.org/10.1520/jfs14705j.

(11) Oommen, Z.; Pierce, S. M. Lead-Free Primer Residues: A Qualitative Characterization of Winchester WinClean (TM), Remington/UMC LeadLess (TM), Federal BallistiClean (TM), and Speer Lawman CleanFire (TM) Handgun Ammunition. J. Forensic Sci. 2006, 51 (3), 509-519. https://doi.org/10.1111/j.1556-4029.2006.00107.x.

(12) Martiny, A.; Campos, A. P. C.; Sader, M. S.; Pinto, M. A. L. SEM/EDS Analysis and Characterization of Gunshot Residues from Brazilian Lead-Free Ammunition. Forensic Sci. Int. 2008, 177 (1), E9-E17. https://doi.org/10.1016/j.forsciint.2007.07.005.

(13) Basu, S. Formation of Gunshot Residues. J. Forensic Sci. 1982, 27 (1), $72-91$. https://doi.org/10.1520/jfs11453j.

(14) Blakey, L. S.; Sharples, G. P.; Chana, K.; Birkett, J. W. Fate and Behavior of Gunshot Residue-A Review. Journal of Forensic Sciences. Blackwell Publishing Inc. January 1, 
2018, pp 9-19. https://doi.org/10.1111/1556-4029.13555.

(15) Hannigan, T. J.; McDermott, S. D.; Greaney, C. M.; O'Shaughnessy, J.; O'Brien, C. M. Evaluation of Gunshot Residue (GSR) Evidence: Surveys of Prevalence of GSR on Clothing and Frequency of Residue Types. Forensic Sci. Int. 2015, 257, 177-181.

(16) Lucas, N.; Cook, M.; Kirkbride, K. P.; Kobus, H. Gunshot Residue Background on Police Officers: Considerations for Secondary Transfer in GSR Evidence Evaluation. Forensic Sci. Int. 2019, 297 (2019), 293-301. https://doi.org/10.1016/j.forsciint.2019.02.017.

(17) Lucas, N.; Seyfang, K. E.; Plummer, A.; Cook, M.; Kirkbride, K. P.; Kobus, H. Evaluation of the Sub-Surface Morphology and Composition of Gunshot Residue Using Focussed Ion Beam Analysis. Forensic Sci. Int. 2019, 297, 100-110. https://doi.org/10.1016/j.forsciint.2019.01.030.

(18) French, J.; Morgan, R. An Experimental Investigation of the Indirect Transfer and Deposition of Gunshot Residue: Further Studies Carried out with SEM-EDX Analysis. Forensic Sci. Int. 2015, 247, 14-17. https://doi.org/10.1016/j.forsciint.2014.10.023.

(19) Gassner, A. L.; Weyermann, C. Prevalence of Organic Gunshot Residues in Police Vehicles. Sci. Justice 2020, 60 (2), 136-144. https://doi.org/10.1016/j.scijus.2019.09.009.

(20) Berk, R. E.; Rochowicz, S. A.; Wong, M.; Kopina, M. A. Gunshot Residue in Chicago Police Vehicles and Facilities: An Empirical Study. J. Forensic Sci. 2007, 52 (4), 838 841. https://doi.org/10.1111/j.1556-4029.2007.00457.x.

(21) Cook, M. Gunshot Residue Contamination of the Hands of Police Officers Following Start-of-Shift Handling of Their Firearm. Forensic Sci. Int. 2016, 269, 56-62. https://doi.org/10.1016/j.forsciint.2016.11.002.

(22) Dalby, O.; Butler, D.; Birkett, J. W. Analysis of Gunshot Residue and Associated Materials: A Review. J. Forensic Sci. 2010, 55 (4), 924-943. https://doi.org/10.1111/j.1556-4029.2010.01370.x.

(23) Chang, K. H.; Jayaprakash, P. T.; Yew, C. H.; Abdullah, A. F. L. Gunshot Residue Analysis and It Evidential Values: A Review. Aust. J. Forensic Sci. 2013, 45 (1), 3-23. https://doi.org/10.1080/00450618.2012.691546.

(24) Koons, R. D.; Havekost, D. G.; Peters, C. A. Analysis of Gunshot Primer Residue Collection Swabs Using Flameless Atomic-Absorption Spectrophotometry and Inductively Coupled Plasma-Atomic Emission-Spectrometry-Effects of a Modified 
Extraction Procedure and Storage of Standards. J. Forensic Sci. 1989, 34 (1), 218-221. https://doi.org/10.1520/jfs12624j.

(25) Koons, R. D. Flameless Atomic Absorption Spectrophotometric Determination of Antimony and Barium in Gunshot Residue Collection Swabs: A Collaborative Study. Crime Lab. Dig. 1993, 20 (1), 19-23.

(26) Comanescu, M. A.; Millett, T. J.; Kubic, T. A. A Study of Background Levels of Antimony, Barium, and Lead on Vehicle Surface Samples by Graphite Furnace Atomic Absorption. J. Forensic Sci. 2019, 64 (2), 565-569. https://doi.org/10.1111/15564029.13899.

(27) Vanini, G.; Souza, R. M.; Destefani, C. A.; Merlo, B. B.; Piorotti, T. M.; de Castro, E. V. R.; Carneiro, M.; Romao, W. Analysis of Gunshot Residues Produced by .38 Caliber Handguns Using Inductively Coupled Plasma-Optical Emission Spectroscopy (ICP OES). Microchem. J. 2014, 115, 106-112. https://doi.org/10.1016/j.microc.2014.03.003.

(28) Costa, R. A.; Motta, L. C.; Destefani, C. A.; Rodrigues, R. R. T.; Santo, K. S. D.; Aquije, G.; Boldrini, R.; Athayde, G. P. B.; Carneiro, M.; Romao, W. Gunshot Residues (GSR) Analysis of Clean Range Ammunition Using SEM/EDX, Colorimetric Test and ICP-MS: A Comparative Approach between the Analytical Techniques. Microchem. J. 2016, 129, 339-347. https://doi.org/10.1016/j.microc.2016.07.017.

(29) Abrego, Z.; Grijalba, N.; Unceta, N.; Maguregui, M.; Sanchez, A.; Fernandez-Isla, A.; Goicolea, M. A.; Barrio, R. J. A Novel Method for the Identification of Inorganic and Organic Gunshot Residue Particles of Lead-Free Ammunitions from the Hands of Shooters Using Scanning Laser Ablation-ICPMS and Raman Micro-Spectroscopy. Analyst 2014, 139 (23), 6232-6241. https://doi.org/10.1039/c4an01051e.

(30) Castellanos, A.; Bell, S.; Fernandez-Lima, F. Characterization of Firearm Discharge Residues Recovered from Skin Swabs Using Sub-Micrometric Mass Spectrometry Imaging. Anal. Methods 2016, 8 (21), 4300-4305. https://doi.org/10.1039/c6ay00096g.

(31) Coumbaros, J.; Kirkbride, K. P.; Klass, G.; Skinner, W. Characterisation of 0.22 Caliber Rimfire Gunshot Residues by Time-of-Flight Secondary Ion Mass Spectrometry (TOFSIMS): A Preliminary Study. Forensic Sci. Int. 2001, 119 (1), 72-81. https://doi.org/https://doi.org/10.1016/S0379-0738(00)00421-7.

(32) Szynkowska, M. I.; Parczewski, A.; Szajdak, K.; Rogowski, J. Examination of Gunshot 
Residues Transfer Using ToF-SIMS. Surf. Interface Anal. 2013, 45 (1), 596-600. https://doi.org/10.1002/sia.5142.

(33) Seyfang, K. E.; Lucas, N.; Redman, K. E.; Popelka-Filcoff, R. S.; Kobus, H. J.; Kirkbride, K. P. Glass-Containing Gunshot Residues and Particles of Industrial and Occupational Origins: Considerations for Evaluating GSR Traces. Forensic Sci. Int. 2019, 298, 284 297. https://doi.org/https://doi.org/10.1016/j.forsciint.2019.03.010.

Ritchie, N. W. M.; DeGaetano, D.; Edwards, D.; Niewoehner, L.; Platek, F.; Wyatt, J. M. Proposed Practices for Validating the Performance of Instruments Used for Automated Inorganic Gunshot Residue Analysis. Forensic Chem. 2020, 20, 100252. https://doi.org/https://doi.org/10.1016/j.forc.2020.100252.

(35) Project FORESIGHT Annual Report, 2017-2018; West Virginia University, 2019.

(36) Feeney, W.; Vander Pyl, C.; Bell, S.; Trejos, T. Trends in Composition, Collection, Persistence, and Analysis of IGSR and OGSR: A Review. Forensic Chem. 2020, 19. https://doi.org/10.1016/j.forc.2020.100250.

Dona-Fernandez, A.; de Andres-Gimeno, I.; Santiago-Toribio, P.; Valtuille-Fernandez, E.; Aller-Sanchez, F.; Heras-Gonzalez, A. Real-Time Detection of GSR Particles from Crime Scene: A Comparative Study of SEM/EDX and Portable LIBS System. Forensic Sci. Int. 2018, 292, 167-175. https://doi.org/10.1016/j.forsciint.2018.09.021. Pyl, C. Vander; Ovide, O.; Ho, M.; Yuksel, B.; Trejos, T. Spectrochemical Mapping Using Laser Induced Breakdown Spectroscopy as a More Objective Approach to Shooting Distance Determination. Spectrochim. Acta Part B At. Spectrosc. 2019, 152, 93-101. https://doi.org/https://doi.org/10.1016/j.sab.2018.12.010. Ott, C. E.; Dalzell, K. A.; Calderon-Arce, P. J.; Alvarado-Gamez, A. L.; Trejos, T.; Arroyo, L. E.; Calderón-Arce, P. J.; Alvarado-Gámez, A. L.; Trejos, T.; Arroyo, L. E. Evaluation of the Simultaneous Analysis of Organic and Inorganic Gunshot Residues Within a Large Population Data Set Using Electrochemical Sensors*(,)Dagger. J. Forensic Sci. 2020, 65 (6), 1935-1944. https://doi.org/10.1111/1556-4029.14548.

(40) Koons, R. D. Analysis of Gunshot Primer Residue Collection Swabs by Inductively Coupled Plasma-Mass Spectrometry. J. Forensic Sci. 1998, 43 (4), 748-754. https://doi.org/10.1520/jfs14301j.

(41) McKenzie-Coe, A.; Bell, S.; Fernandez-Lima, F. Detection of Firearm Discharge Residue 
from Skin Swabs Using Trapped Ion Mobility Spectrometry Coupled to Mass Spectrometry. Anal. Methods 2018, 10 (35), 7. https://doi.org/10.1039/c8ay00658j.

(42) Taudte, R. V.; Beavis, A.; Blanes, L.; Cole, N.; Doble, P.; Roux, C. Detection of Gunshot Residues Using Mass Spectrometry. BioMed. Res. Int. 2014, 2014 (3), 1-16. https://doi.org/10.1155/2014/965403.

(43) Eckenrode, B. A. Environmental and Forensic Applications of Field-Portable GC-MS: An Overview. J. Am. Soc. Mass. Spectrom. 2001, 12 (6), 683-693. https://doi.org/10.1016/S1044-0305(01)00251-3.

(44) Morelato, M.; Beavis, A.; Ogle, A.; Doble, P.; Kirkbride, P.; Roux, C. Screening of Gunshot Residues Using Desorption Electrospray Ionisation-Mass Spectrometry (DESIMS). Forensic Sci. Int. 2012, 217 (1-3), 101-106. https://doi.org/10.1016/j.forsciint.2011.10.030.

(45) Mason, K.; Wuhrer, R. Detection and Characterization of Heavy-Metal-Free (HMF) Gunshot Residues Using CL, EDS and Raman Together with XRD. Mircoscopic Microanal. 2018, 24 (1), 1174-1175. https://doi.org/10.1017/S1431927618006359.

(46) Bueno, J.; Lednev, I. K. Advanced Statistical Analysis and Discrimination of Gunshot Residue Implementing Combined Raman and FT-IR Data Electronic Supplementary Information (ESI) Available. See DOI: 10.1039/C3ay40721g. Anal. Methods 2013, 5 (22), 6292-6296. https://doi.org/10.1039/c3ay40721g.

(47) López-López, M.; Delgado, J. J.; García-Ruiz, C. Ammunition Identification by Means of the Organic Analysis of Gunshot Residues Using Raman Spectroscopy. Anal. Chem. 2012, 84 (8), 3581-3585. https://doi.org/10.1021/ac203237w.

(48) Doty, K. C.; Lednev, I. K. Raman Spectroscopy for Forensic Purposes: Recent Applications for Serology and Gunshot Residue Analysis. Trends Anal. Chem. 2018, 103, 215-222. https://doi.org/10.1016/j.trac.2017.12.003.

(49) Rinke-Kneapler, C. N.; Sigman, M. E. Applications of Laser Spectroscopy in Forensic Science. In Laser Spectroscopy for Sensing: Fundamentals, Techniques and Applications; Baudelet, M., Ed.; Woodhead Publ Ltd: Cambridge, 2014; pp 461-495. https://doi.org/10.1533/9780857098733.3.461.

(50) Rosenberg, M. B.; Dockery, C. R. Determining the Lifetime of Detectable Amounts of Gunshot Residue on the Hands of a Shooter Using Laser-Induced Breakdown 
Spectroscopy. Appl. Spectrosc. 2008, 62 (11), 1238-1241.

https://doi.org/10.1366/000370208786401473.

(51) Lopez-Lopez, M.; Alvarez-Llamas, C.; Pisonero, J.; Garcia-Ruiz, C.; Bordel, N. An Exploratory Study of the Potential of LIBS for Visualizing Gunshot Residue Patterns. Forensic Sci. Int. 2017, 273, 124-131. https://doi.org/10.1016/j.forsciint.2017.02.012.

(52) Tarifa, A.; Almirall, J. R. Fast Detection and Characterization of Organic and Inorganic Gunshot Residues on the Hands of Suspects by CMV-GC-MS and LIBS. Sci. Justice 2015, 55 (3), 168-175. https://doi.org/10.1016/j.scijus.2015.02.003.

(53) Trejos, T.; Vander Pyl, C.; Menking-Hoggatt, K.; Alvarado, A. L.; Arroyo, L. E. Fast Identification of Inorganic and Organic Gunshot Residues by LIBS and Electrochemical Methods . Forensic Chem. 2018, 8, 146-156. https://doi.org/doi.org/10.1016/j.forc.2018.02.006.

(54) Silva, M. J.; Cortez, J.; Pasquini, C.; Honorato, R. S.; Paima, A. P. S.; Pimentel, M. F.; Paim, A. P. S.; Pimentel, M. F. Gunshot Residues: Screening Analysis by Laser-Induced Breakdown Spectroscopy. J. Braz. Chem. Soc. 2009, 20 (10), 1887-1894. https://doi.org/10.1590/S0103-50532009001000017.

(55) Martin, M. Z.; Labbe, N.; Andre, N.; Harris, R.; Ebinger, M.; Wullschleger, S. D.; Vass, A. A. High Resolution Applications of Laser-Induced Breakdown Spectroscopy for Environmental and Forensic Applications. Spectrochim. Acta Part B-Atomic Spectrosc. 2007, 62 (12), 1426-1432. https://doi.org/10.1016/j.sab.2007.10.046.

(56) Dockery, C. R.; Goode, S. R. Laser-Induced Breakdown Spectroscopy for the Detection of Gunshot Residues on the Hands of a Shooter. Appl. Opt. 2003, 42 (30), 6153-6158. https://doi.org/10.1364/ao.42.006153.

(57) Menking-Hoggatt, K.; Arroyo, L.; Curran, J.; Trejos, T. Novel LIBS Method for MicroSpatial Chemical Analysis of Inorganic Gunshot Residue. J. Chemom. 2019, e3208, 13.

(58) Hearns, N. G. R.; Lafleche, D. N.; Sandercock, M. L. Preparation of a Ytterbium-Tagged Gunshot Residue Standard for Quality Control in the Forensic Analysis of GSR. J. Forensic Sci. 2015, 60 (3), 737-742. https://doi.org/10.1111/1556-4029.12729.

(59) Schumacher, R.; Barth, M.; Neimke, D.; Niewoehner, L. Investigation of Gunshot Residue Patterns Using Milli-XRF-Techniques: First Experiences in Casework; 2010; Vol. 7729. https://doi.org/10.1117/12.853852. 
(60) Charles, S.; Dodier, T.; Kaindl, M.; Kastéropoulos, A.; Knijnenberg, A.; Larsson, M.; Lauper, S.; Merat, N.; Niewoehner, L.; Scholz, T.; Simon, L. Conduction of a RoundRobin Test on a Real Sample for the Identification of Gunshot Residues by SEM/EDX. Forensic Sci. Int. 2020, 309. https://doi.org/10.1016/j.forsciint.2020.110183.

(61) Planotec GSR \& Particle Analysis Calibration Kit https://www.tedpella.com/calibration_html/Gunshot_Particle_Analysis_Calibration_Kit.ht m.

(62) GSR and particle analysis calibration kit http://www.agarscientific.com/particle-analysisstandard.

(63) Menking-Hoggatt, K.; Martinez, C.; Vander Pyl, C.; Heller, E.; Pollock, E. “Chip”; Arroyo, L.; Trejos, T. Development of Tailor-Made Inorganic Gunshot Residue (IGSR) Microparticle Standards and Characterization with a Multi-Technique Approach. Talanta 2021. https://doi.org/10.1016/j.talanta.2020.121984. 


\subsection{Expanded Characterization of the Microparticle IGSR Standards}

\subsubsection{Characterization of Tailor-Made IGSR Standards}

The 2020 Talanta manuscript ${ }^{63}$ served as a proof of principle for the development of tailormade materials with physical and chemical composition of gunshot residues. The preliminary characterization of ten tailor-made IGSR standard was expanded to twenty-five standard materials, representing pGSR from 15 leaded and 10 lead-free ammunition, and a full list can be found in Table 5. The method of characterization and analysis remained the same as described in section 1.3. The characterization was also continued to 24 and 52 weeks for 15 out of 20 primer with three stock replicate suspensions collected for each primer. One set of five lead-free primers was characterized for only 24 weeks due to the original primers purchased not being representative of their lead-free advertised information.

As discussed previously, the study was designed to assess the stability of the inorganic elemental composition of the p-GSR standards once they are suspended in the organic solution and being stored in the laboratory for extended periods. When the primer-only GSR microparticles were produced, each primer type was collected three times, each one originating from the firing of a separate cartridge/primer (replicate A first, B second, and C third). The general chemical profile between each cartridge/primer of the same ammunition is anticipated to be similar. However, because the number of particles recovered can vary between firings, each replicate (A, B, C) is treated as a single stock suspension with each own characterized composition and particle counts. The stock microparticle samples are characterized and in analyzed by ICP-MS in triplicates, resulting in nine samples per ammunition type. The triplicate samples are repeated at the week intervals specified for the stability study. Once ICP-MS analysis is completed, the concentrations calculated by the ICP-MS Mass Hunter 4.4 software (version C.01.04) are exported for further statistical analysis using both Excel (Microsoft 365) and JMP Pro 14.0.0.

Analysis of Variance (ANOVA) was performed on the estimated concentrations to determine the stability of the elemental composition for the in-house primer-only standards while being stored over the one-year experiment. In this study, the data was grouped by a factor of interest (i.e., week $0,2,6,12,24$, and 52 ) to evaluate the between and within group variation. A $99 \%$ confidence interval ( $\alpha$ value 0.01 ) was selected for the stability assessment. The primer-only standards were 
considered stable if no significant difference in the elemental concentrations was observed over time. If a significant difference was observed, then a post-HOC Tukey-Kramer test was used to determine which of the samples varied in the collection.

Figure 8 is an example of one primer replicate (B) of a standard ammunition (TULAMMO) and a lead-free ammunition (Inceptor) evaluated over the one-year. The line in the center of the graphs represents the overall mean between the groups, the line in the center of the diamonds represents the individual group mean, and the vertical span represents the $99 \%$ confidence interval. As previously explained, similar size circles confirm comparable variance across weeks, and the overlapping circles aids to visualize the variation between time snapshots. All the elements above LOQ showed no significant difference in the elemental concentrations over time. Interesting to note, we observed some trends in the variation of concentration over time within the same ammunition stock suspension as illustrated in Figure 8 (i.e., $\mathrm{Pb}$ and $\mathrm{Sb}$ in the TUL and Ti and $\mathrm{Zn}$ in the INC). The common variation patterns may be indicative of uncontrolled variables such as slight solvent evaporation, settling of some particles, or minor differences in the number or size of particles collected per aliquot between sampled weeks, causing small increases or decreases on the composition. Nonetheless, the correlation of elements on each formulation, indicates that relative ratios (e.g., $\mathrm{Pb} / \mathrm{Sb}, \mathrm{Ti} / \mathrm{Zn}$ ) can be used as a normalization method to minimize the between-week variability.

This analysis was performed for thirty leaded and thirty non-leaded stock primer suspensions for the elemental concentrations above the LOQ and the average results for the oneyear study is shown in Table 3. The elements had between 4-20\% inter-week variation, except aluminum, which has shown more variation in both the ICP-MS data and in the particle's composition observed by SEM-EDS (section 4.4.2.). SEM-EDS has shown that some IGSR particles contain aluminum or copper, while others do not. These elements are seen at concentrations ranging between the LOD and LOQ by ICP-MS, meaning the presences of the elements in the IGSR standards are variable and in low concentration.

Table 6 displays only the replicates measurement for one primer-only sample replicate (B) for each of the collected ammunition type. Five of the lead-free primers are only at week 24 in the stability study, as marked by asterisks by the primer type. Inter-week reproducibility between week $0,6,12,24$, and 52 was below $20 \%$ for most elements of interest. The study demonstrates that 
Table 5: List of the different primer types collected for characterization of the tailor-made IGSR standards. Each primer type was collected in triplicate.

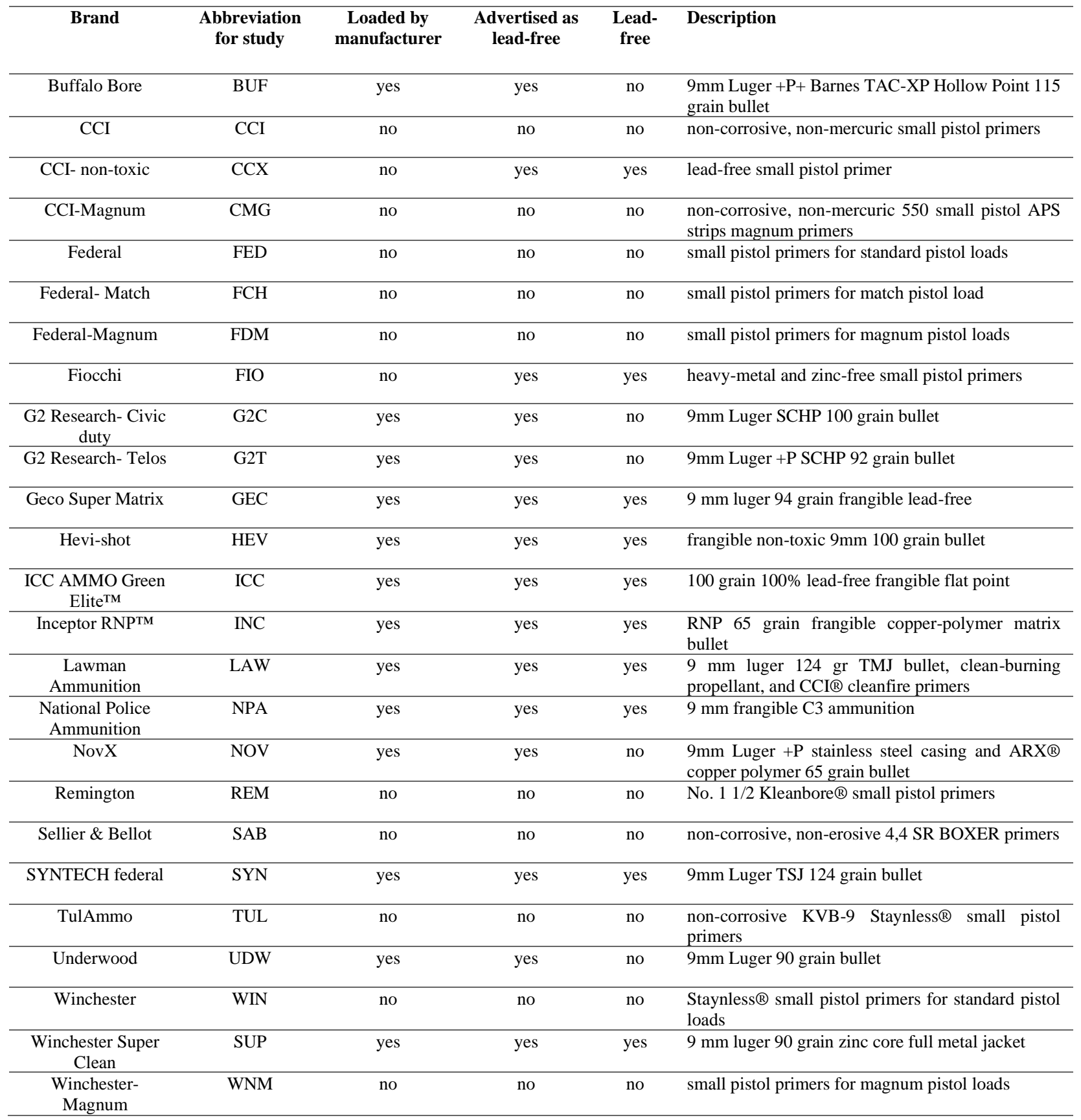


TULAMMO primer-only

Replicate B
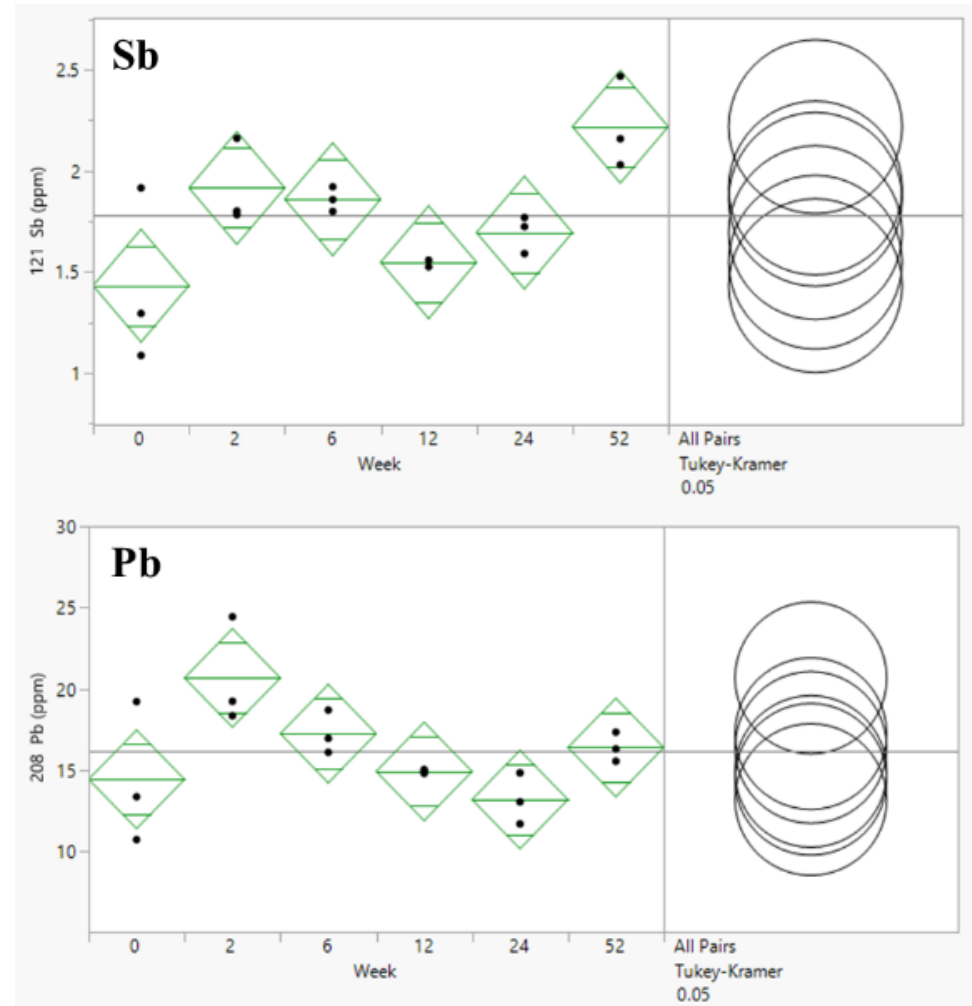

Inceptor primer-only

Replicate B
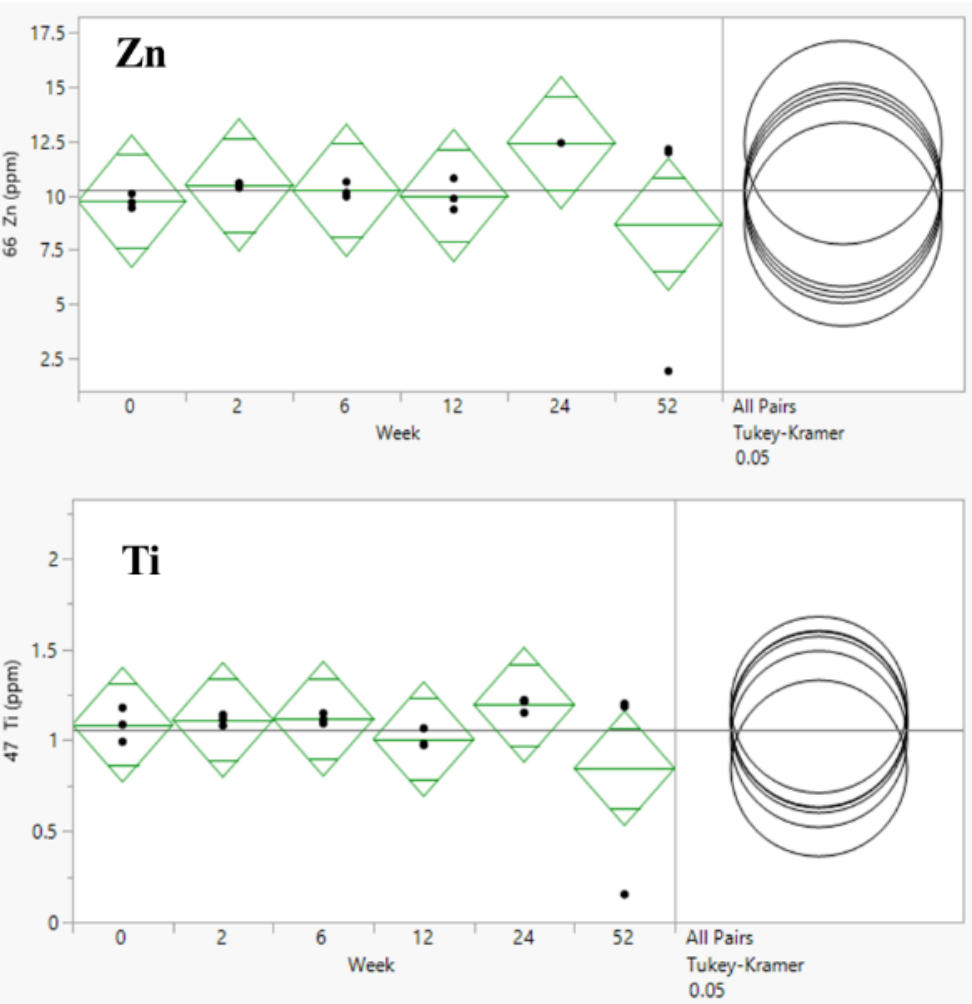

Figure 8: ANOVA and Tukey-Kramer for two IGSR in-house primer-only standards. The results confirm the elements of interest (possible GSR markers) are remaining stable during the one-year storage period. 
each stock suspension of microparticles remain stable at room temperature for up to one year. It was observed however, that ANOVA found a statistical difference between the replicates of the same primer type from different primer discharges (i.e. replicate stock suspensions A, B, C). This is to be expected because the process of discharging the primer can vary due to the uncontrolled variables. The difference in the firing pin striking the primer, both in angle and force, can affect the discharging process. It is experimentally difficult, if not improbable, to reproduce the number of microscopic GSR particles produced in a firing event and then deposited on the collecting flask.

Also, the individual boxes of primers do not necessarily always originate from the same manufacturing lot. When stored properly, a primer has a very long shelf-life, so large batches can be made and stored until needed. The downside to this is that primers of different batches can be mixed into the same box of ammunition. However, within each stock microparticle suspension the composition and particles remain stable. Regardless of the between-cartridges variation of the number of IGSR particles collected during the in-house standard method, the stability observed on a single cartridge/primer standard solution serves as proof of principle that the proposed method can be used to create leaded and lead-free IGSR standards. From a practical perspective, only few microliters of the standard are needed for instrumental detection and therefore each stock suspension can generate thousands of dry-standards and/or be used in many experiments in its liquid form. As a result, these suspensions fit for use as standard laboratory and have the potential to be used in future method validations, as quality controls, and for interlaboratory studies.

Overall, the lead-free primers are found in various elemental combinations, while the standard ammunition have relatively similar elemental profiles, as can be seen in Table 6. For example, common profiles observed in lead-free ammunition were tricomponent $\mathrm{Al} / \mathrm{Si} / \mathrm{K}$ particles, binary $\mathrm{Zn} / \mathrm{Ti}$ particles or single element particles containing, $\mathrm{Ba}, \mathrm{Bi}$, Sr or K. In contrast, leaded ammunition presented the expected $\mathrm{Pb} / \mathrm{Ba} / \mathrm{Sb}$ composition, with the exception of Tulamo $(\mathrm{Pb} / \mathrm{Sb})$. These preliminary results highlight the relevance of characterizing IGSR primers to provide better tools for the interpretation of GSR evidence, as the composition of modern ammunition is relatively variable.

Deposition and analysis by SEM-EDS of the in-house primer-only standards was performed at $0,6,12$, and 52-weeks after the acetone suspension was created for each of the ten leaded and five lead-free collected primers monitored for a year, and only to week 12 for the five 
Table 6: Summary of the elemental concentration for the ten leaded and five lead-free IGSR-primer standards collected and values represent mean concentrations observed over 52-weeks. Those with an asterisk (*) have only been monitored for 24-weeks and are still being monitored.

\begin{tabular}{|c|c|c|c|c|c|c|c|c|c|c|}
\hline \multirow[b]{2}{*}{ Element } & \multicolumn{10}{|c|}{ Lead-free primer types and concentrations for replicate $B(\mathbf{p p m})$} \\
\hline & CCX & FIO & HEV & INC & SYN & GEC * & ICC* & LAW* & NPA* & SUP* \\
\hline B & - & - & - & - & - & - & - & - & - & - \\
\hline $\mathbf{K}$ & - & $8.5 \pm 1.2$ & - & - & - & - & - & - & $8.4 \pm 0.9$ & $3.5 \pm 0.7$ \\
\hline $\mathbf{C a}$ & - & - & - & - & - & - & - & $5.6 \pm 0.5$ & - & - \\
\hline $\mathbf{T i}$ & - & - & - & $1.2 \pm 0.1$ & - & $0.88 \pm 0.19$ & $1.0 \pm 0.2$ & - & - & - \\
\hline $\mathbf{C u}$ & - & $0.27 \pm 0.03$ & - & - & - & - & - & - & - & - \\
\hline Zn & - & - & - & $10.7 \pm 0.9$ & - & $6.5 \pm 1.2$ & $7.9 \pm 0.8$ & - & - & - \\
\hline $\mathbf{S r}$ & - & - & - & - & - & - & - & $5.7 \pm 0.4$ & - & - \\
\hline Sn & & & & & & - & - & - & - & - \\
\hline $\mathbf{B a}$ & $3.3 \pm 0.4$ & - & $5.9 \pm 0.8$ & - & - & - & - & - & - & - \\
\hline \multirow[t]{2}{*}{$\mathbf{B i}$} & - & $0.05 \pm 0.01$ & $0.01 \pm 0.01$ & $0.01 \pm 0.00$ & $0.69 \pm 0.14$ & - & - & - & - & - \\
\hline & \multicolumn{10}{|c|}{ Leaded primer types and concentrations for replicate $B(p p m)$} \\
\hline Element & FED & REM & SAB & TUL & WIN & CCI & CMG & $\mathrm{FCH}$ & FDM & WNM \\
\hline Sb & $3.4 \pm 0.5$ & $1.5 \pm 0.2$ & $1.1 \pm 0.2$ & $1.8 \pm 0.3$ & $3.0 \pm 0.4$ & $2.8 \pm 0.3$ & $3.2 \pm 0.5$ & $3.4 \pm 0.4$ & $3.1 \pm 0.4$ & $2.2 \pm 0.2$ \\
\hline $\mathbf{B a}$ & $12.0 \pm 1.9$ & $8.1 \pm 1.5$ & $3.7 \pm 0.6$ & - & $6.0 \pm 1.1$ & $8.0 \pm 1.0$ & $9.6 \pm 1.1$ & $8.4 \pm 1.0$ & $10.1 \pm 1.6$ & $6.6 \pm 1.0$ \\
\hline $\mathbf{P b}$ & $7.6 \pm 1.6$ & $2.6 \pm 0.5$ & $2.5 \pm 0.5$ & $16.1 \pm 3.2$ & $4.7 \pm 1.1$ & $2.6 \pm 0.4$ & $3.3 \pm 0.3$ & $8.3 \pm 0.7$ & $7.2 \pm 1.1$ & $2.4 \pm 0.2$ \\
\hline
\end{tabular}


remaining lead-free primers. One of the disadvantages of SEM-EDS is the time-consuming nature and has proven to be the bottle neck of our research study. Therefore, only one primer stock replicate (e.g. replicate B) in the collection process was analyzed. The analysis consisted of four deposits for each of the 20 primer types which resulted in a total of 80 samples to be analyzed by SEM-EDS. To date, fifteen primers have been monitored by SEM-EDS by imaging and acquiring EDS spectra for ten particles per stub, instead of performing a full automated mapping. This first stage allowed the chemical and morphological characterization of the microparticle standards. The second step of this study consists of the particle count of the various suspensions. The chemical mapping and particle count experiment will be performed preliminarily on one leaded and one lead-free primer type.

When analyzing the primer-only samples, the SEM-EDS data was reviewed by the analyst. This allowed the elemental composition of the primer-only samples to be determined and the morphology of the particles to be evaluated. An important part of GSR analysis is the confirmation of the elemental composition within a micrometer-sized spheroid particle. The spheroid morphology is important because of how GSR is created during the firing process. When a firearm is discharged, the ammunition undergoes a rapid, high temperature and pressure thermodynamic process as it expels the bullet from the barrel. The plume of the created GSR is subjected to rapid heating and cooling, causing the molten components to condense and form spherical particles. This process of spherical particle formation can be explained by the cohesive forces applied as the particle cools. ${ }^{54}$

The in-house primer-only standard exhibits the same spherical morphology that is expected when analyzing GSR. Figure 9 provides an example of a leaded ammunition with the typical GSR morphology; interestingly, the particles in this standard ammunition did not have barium as part of the elemental composition, which was corroborated by ICP-MS and LIBS. If this type of ammunition is treated as evidence, the interpretation would result in a "consistent with" result since it does not have the right combination of elements expected $(\mathrm{Pb}, \mathrm{Ba}, \mathrm{Sb})$ for a higher confidence classification of "characteristic with GSR" standard ammunition. So, even though the TULAMMO ammunition is known to originate from a leaded ammunition in this study, the composition is not characteristic of a GSR particle.

On the other hand, Figure 10 provides an example of a lead-free (lead-free) primer with an irregular morphology and no elements that would identify the particle as characteristic or 
consistent with GSR. An item of evidence using this type of ammunition would not be identified as GSR based on the current ASTM standard criteria, demonstrating the relevance of this study in increasing the knowledge of morphology and chemical composition of diverse modern ammunition.

The results from the particles identified by SEM-EDS analysis are also in agreement with the elemental profiles observed in task 1.2 by ICP-MS, providing a greater level of confidence in the conclusions for the different primers. While ICP-MS cannot provide morphology information, the elemental composition can be determined quantitatively with high sensitivity, reproducibility, and accuracy. These figures of merit are critical for the characterization of these microparticle suspensions for its use as matrix-matched quality control standards. Also, the use of complementary methods is important when analyzing the modern ammunition to expand the information of GSR composition commonly reported in literature. Table 7 provides a summary of the elements identified by SEM-EDS for the different types of ammunition.

Lastly, LIBS was completed to provide another set of complementary elemental information and to demonstrate the application of the in-house IGSR microparticle standards. A $100 \mathrm{uL}$ aliquot of one replicate for each type of IGSR microparticle standard was spiked on a typical GSR stub and left to dry, leaving only microparticles behind. The stubs were analyzed with the rapid micro-spot LIBS method validated in our research group last year. Each spectrum collected was cropped, background subtracted, and integrated for the elements of interest and the signal to noise for each element was calculated with a background close to each emission line of interest.

Table 8 is a summary of the elements detected by each analytical method. There was agreement in the elemental compositions across the different techniques. One difference seen in the LIBS data when comparing the elemental compositions was in the detection of bismuth. SEMEDS and ICP-MS have lower LOD than LIBS (single particle 1\% w/w, 1 ppb, and $\sim 1 \mathrm{ppm}$ respectively); therefore, LIBS was unable to detect bismuth in the SYN ammunition. This study constitutes the first step to provide the scientific community with access to an IGSR standard. Our next steps will be to determine reproducible particle count and to conduct interlaboratory studies with our collaborators. 


\section{TULAMMO}

a standard ammunition without $\mathrm{Ba}$

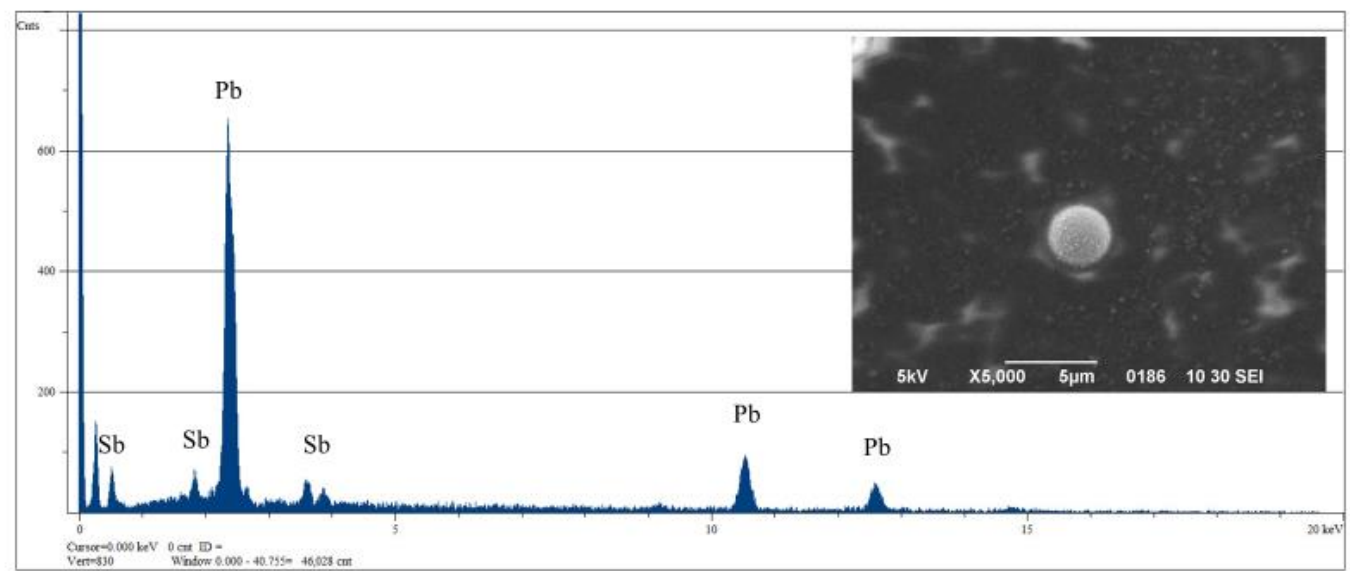

Figure 9: Example of a primer-only particle generated by TULAMMO: standard, leaded ammunition that lacks barium.

Fiocchi

lead-free ammunition with only light elements

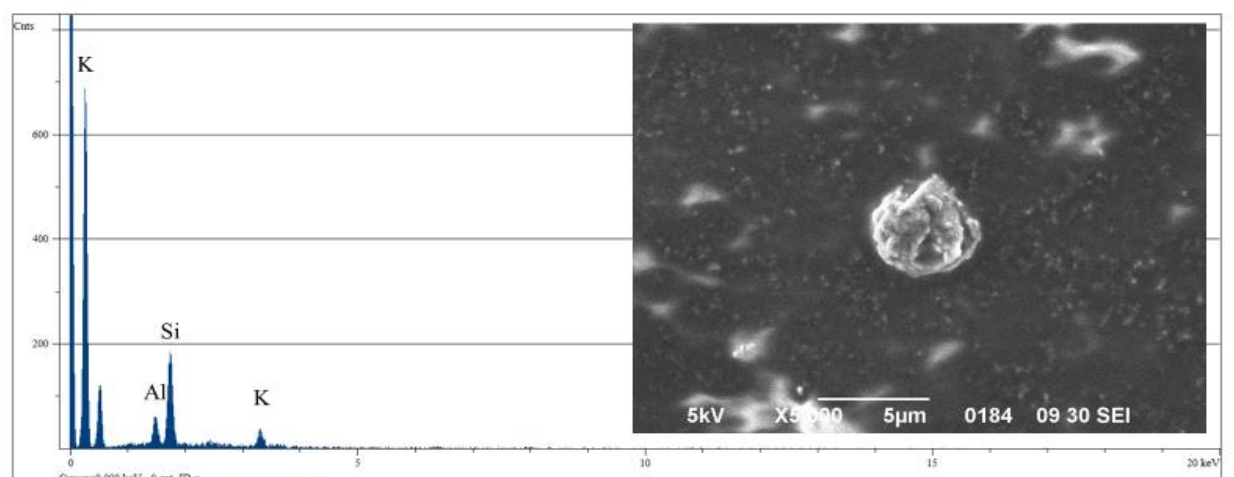

Figure 10: Example of particle generated by Fiocchi: nontoxic ammunition that lacks any characteristic GSR markers. 
Table 7: Summary of the elements observed by SEM-EDS analysis.

\begin{tabular}{|c|c|c|c|c|c|c|c|}
\hline \multicolumn{4}{|c|}{ Lead-free Ammunition } & \multicolumn{4}{|c|}{ Standard Ammunition } \\
\hline Primer type & $\begin{array}{l}\text { Present in all } \\
\text { Particles }\end{array}$ & $\begin{array}{l}\text { Present in Some } \\
\text { Particles }\end{array}$ & $\begin{array}{l}\text { Confirmed by } \\
\text { ICP-MS }\end{array}$ & Primer Type & $\begin{array}{l}\text { Present in all } \\
\text { Particles }\end{array}$ & $\begin{array}{l}\text { Present in Some } \\
\text { Particles }\end{array}$ & $\begin{array}{l}\text { Confirmed by } \\
\text { ICP-MS }\end{array}$ \\
\hline CCX & $\mathrm{Ba}$ & $\mathrm{Al}, \mathrm{Si}, \mathrm{K}$ & yes & $\mathrm{CCI}$ & $\mathrm{Sb}, \mathrm{Pb}, \mathrm{Ba}$ & $\mathrm{Al}$ & yes \\
\hline FIO & $\mathrm{Al}, \mathrm{Si}, \mathrm{K}$ & $\mathrm{Bi}$ & yes & CMG & $\mathrm{Sb}, \mathrm{Pb}, \mathrm{Ba}$ & $\mathrm{Al}$ & yes \\
\hline HEV & $\mathrm{Ba}$ & $\mathrm{Al}, \mathrm{Si}, \mathrm{K}, \mathrm{W}$ & yes & FCH & $\mathrm{Sb}, \mathrm{Pb}, \mathrm{Ba}$ & - & yes \\
\hline INC & $\mathrm{Zn}, \mathrm{Ti}$ & - & yes & FDM & $\mathrm{Sb}, \mathrm{Pb}, \mathrm{Ba}$ & $\mathrm{Al}, \mathrm{Si}$ & yes \\
\hline SYN & $\mathrm{Bi}$ & $\mathrm{Al}$ & yes & FED & $\mathrm{Sb}, \mathrm{Pb}, \mathrm{Ba}$ & - & yes \\
\hline GEC & $\mathrm{Ti}, \mathrm{Zn}$ & - & yes & REM & $\mathrm{Sb}, \mathrm{Pb}, \mathrm{Ba}$ & $\mathrm{Al}$ & yes \\
\hline ICC & $\mathrm{Ti}, \mathrm{Zn}$ & - & yes & SAB & $\mathrm{Sb}, \mathrm{Pb}, \mathrm{Ba}$ & - & yes \\
\hline LAW & $\mathrm{Sr}$ & $\mathrm{Ca}$ & yes & TUL & $\mathrm{Sb}, \mathrm{Pb}$ & $\mathrm{Al}, \mathrm{Si}$ & yes \\
\hline NPA & $\mathrm{K}$ & - & yes & WIN & $\mathrm{Sb}, \mathrm{Pb}, \mathrm{Ba}$ & - & yes \\
\hline SUP & K & $\mathrm{Sn}$ & yes & WNM & $\mathrm{Sb}, \mathrm{Pb}, \mathrm{Ba}$ & $\mathrm{Al}$ & yes \\
\hline
\end{tabular}


Table 8: Summary of the elements detected in the IGSR microparticle solutions by all techniques for different primers.

\begin{tabular}{|c|c|c|c|c|c|}
\hline Primer Brand Name & $\begin{array}{c}\text { Primer } \\
\text { Abbreviation }\end{array}$ & $\begin{array}{c}\text { Non- } \\
\text { Leaded }\end{array}$ & ICP-MS & SEM-EDS & LIBS \\
\hline CCI- lead-free & CCX & yes & $\mathrm{Ba}$ & $\mathrm{Ba}$ & $\mathrm{Ba}$ \\
\hline Fiocchi & FIO & yes & $\mathrm{K}, \mathrm{Cu}$ & $\mathrm{K}, \mathrm{Cu}$ & $\mathrm{K}, \mathrm{Cu}$ \\
\hline Hevi-shot & $\mathrm{HEV}$ & yes & $\mathrm{Ba}$ & $\mathrm{Ba}$ & $\mathrm{Ba}$ \\
\hline Inceptor RNPTM & INC & yes & $\mathrm{Ti}, \mathrm{Zn}$ & $\mathrm{Ti}, \mathrm{Zn}$ & $\mathrm{Ti}, \mathrm{Zn}$ \\
\hline SYNTECH federal & SYN & yes & $\mathrm{Bi}$ & $\mathrm{Bi}$ & - \\
\hline Geco Super Matrix & GEC & yes & $\mathrm{Ti}, \mathrm{Zn}$ & $\mathrm{Ti}, \mathrm{Zn}$ & $\mathrm{Ti}, \mathrm{Zn}$ \\
\hline $\begin{array}{l}\text { ICC AMMO Green } \\
\text { Elite }^{\mathrm{TM}}\end{array}$ & $\mathrm{ICC}$ & yes & $\mathrm{Ti}, \mathrm{Zn}$ & $\mathrm{Ti}, \mathrm{Zn}$ & $\mathrm{Ti}, \mathrm{Zn}$ \\
\hline $\begin{array}{l}\text { Lawman } \\
\text { Ammunition }\end{array}$ & LAW & yes & $\mathrm{Ca} \mathrm{Sr}$ & $\mathrm{Sr}$ & $\mathrm{Sr}$ \\
\hline $\begin{array}{l}\text { National Police } \\
\text { Ammunition }\end{array}$ & NPA & yes & $\mathrm{K}$ & $\mathrm{K}$ & $\mathrm{K}$ \\
\hline $\begin{array}{l}\text { Winchester Super } \\
\text { Clean }\end{array}$ & SUP & yes & K Sn & $\mathrm{K}$ & K \\
\hline Buffalo Bore & BUF & no & $\mathrm{Sb}, \mathrm{Pb}, \mathrm{Ba}$ & $\mathrm{Sb}, \mathrm{Pb}, \mathrm{Ba}$ & $\mathrm{Sb}, \mathrm{Pb}, \mathrm{Ba}$ \\
\hline CCI & CCI & no & $\mathrm{Sb}, \mathrm{Pb}, \mathrm{Ba}$ & $\mathrm{Sb}, \mathrm{Pb}, \mathrm{Ba}$ & $\mathrm{Sb}, \mathrm{Pb}, \mathrm{Ba}$ \\
\hline CCI-Magnum & CMG & no & $\mathrm{Sb}, \mathrm{Pb}, \mathrm{Ba}$ & $\mathrm{Sb}, \mathrm{Pb}, \mathrm{Ba}$ & $\mathrm{Sb}, \mathrm{Pb}, \mathrm{Ba}$ \\
\hline Federal & FED & no & $\mathrm{Sb}, \mathrm{Pb}, \mathrm{Ba}$ & $\mathrm{Sb}, \mathrm{Pb}, \mathrm{Ba}$ & $\mathrm{Sb}, \mathrm{Pb}, \mathrm{Ba}$ \\
\hline Federal- Match & $\mathrm{FCH}$ & no & $\mathrm{Sb}, \mathrm{Pb}, \mathrm{Ba}$ & $\mathrm{Sb}, \mathrm{Pb}, \mathrm{Ba}$ & $\mathrm{Sb}, \mathrm{Pb}, \mathrm{Ba}$ \\
\hline Federal-Magnum & FDM & no & $\mathrm{Sb}, \mathrm{Pb}, \mathrm{Ba}$ & $\mathrm{Sb}, \mathrm{Pb}, \mathrm{Ba}$ & $\mathrm{Sb}, \mathrm{Pb}, \mathrm{Ba}$ \\
\hline $\begin{array}{l}\text { G2 Research- Civic } \\
\text { duty }\end{array}$ & $\mathrm{G} 2 \mathrm{C}$ & no & $\mathrm{Sb}, \mathrm{Pb}, \mathrm{Ba}$ & $\mathrm{Sb}, \mathrm{Pb}, \mathrm{Ba}$ & $\mathrm{Sb}, \mathrm{Pb}, \mathrm{Ba}$ \\
\hline G2 Research- Telos & $\mathrm{G} 2 \mathrm{~T}$ & no & $\mathrm{Sb}, \mathrm{Pb}, \mathrm{Ba}$ & $\mathrm{Sb}, \mathrm{Pb}, \mathrm{Ba}$ & $\mathrm{Sb}, \mathrm{Pb}, \mathrm{Ba}$ \\
\hline NovX & NOV & no & $\mathrm{Sb}, \mathrm{Pb}, \mathrm{Ba}$ & $\mathrm{Sb}, \mathrm{Pb}, \mathrm{Ba}$ & $\mathrm{Sb}, \mathrm{Pb}, \mathrm{Ba}$ \\
\hline Remington & REM & no & $\mathrm{Sb}, \mathrm{Pb}, \mathrm{Ba}$ & $\mathrm{Sb}, \mathrm{Pb}, \mathrm{Ba}$ & $\mathrm{Sb}, \mathrm{Pb}, \mathrm{Ba}$ \\
\hline Sellier \& Bellot & SAB & no & $\mathrm{Sb}, \mathrm{Pb}, \mathrm{Ba}$ & $\mathrm{Sb}, \mathrm{Pb}, \mathrm{Ba}$ & $\mathrm{Sb}, \mathrm{Pb}, \mathrm{Ba}$ \\
\hline TulAmmo & TUL & no & $\mathrm{Sb}, \mathrm{Pb}$ & $\mathrm{Sb}, \mathrm{Pb}$ & $\mathrm{Sb}, \mathrm{Pb}$ \\
\hline Underwood & UDW & no & $\mathrm{Sb}, \mathrm{Pb}, \mathrm{Ba}$ & $\mathrm{Sb}, \mathrm{Pb}, \mathrm{Ba}$ & $\mathrm{Sb}, \mathrm{Pb}, \mathrm{Ba}$ \\
\hline Winchester & WIN & no & $\mathrm{Sb}, \mathrm{Pb}, \mathrm{Ba}$ & $\mathrm{Sb}, \mathrm{Pb}, \mathrm{Ba}$ & $\mathrm{Sb}, \mathrm{Pb}, \mathrm{Ba}$ \\
\hline Winchester-Magnum & WNM & no & $\mathrm{Sb}, \mathrm{Pb}, \mathrm{Ba}$ & $\mathrm{Sb}, \mathrm{Pb}, \mathrm{Ba}$ & $\mathrm{Sb}, \mathrm{Pb}, \mathrm{Ba}$ \\
\hline
\end{tabular}




\section{Section 5}

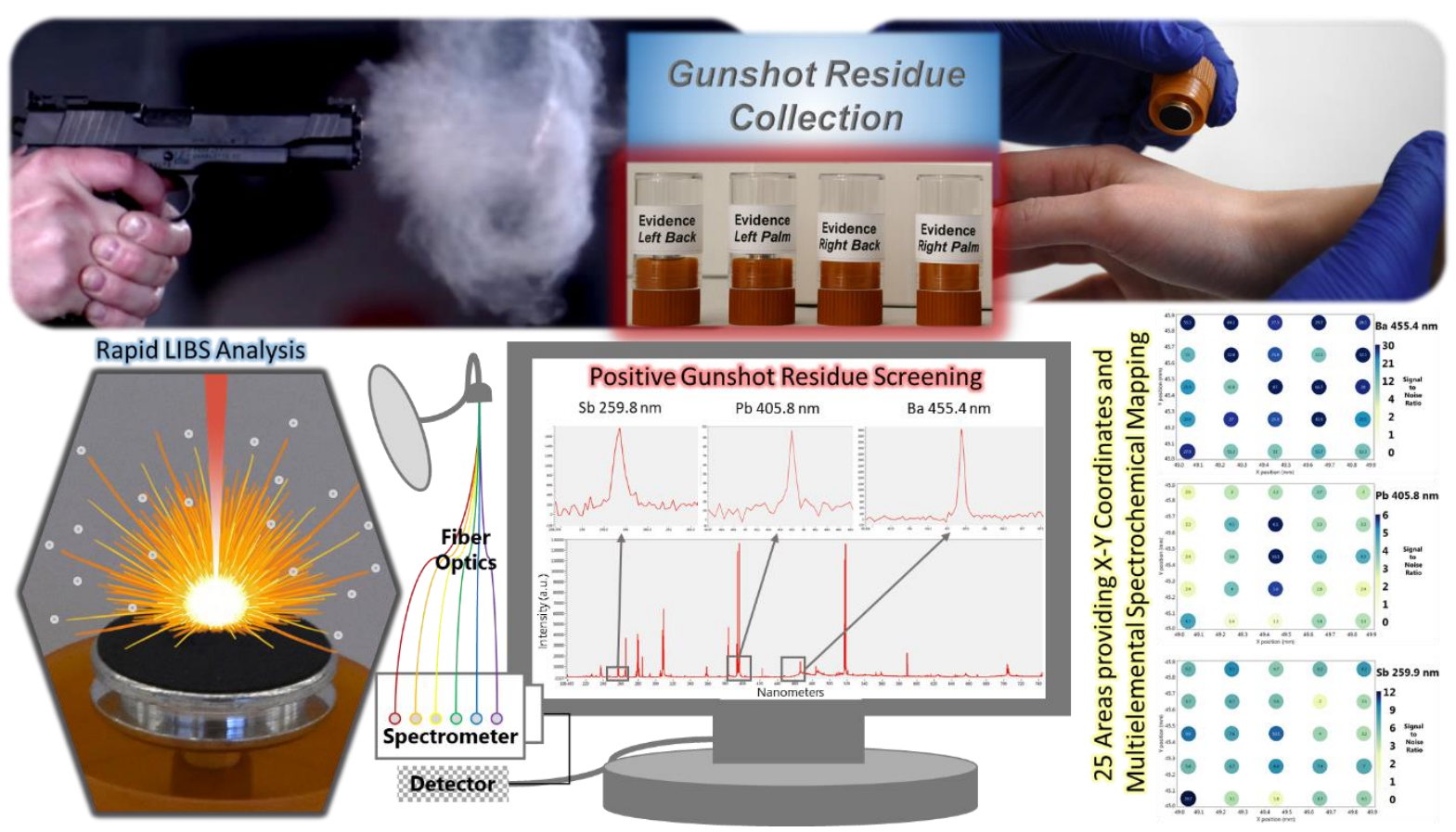

Chapter 3

Development of the Rapid LIBS Micro-Spatial Method for the Detection of IGSR 


\section{Chapter 3: Development of the Rapid LIBS Micro-Spatial Method for the Detection of IGSR}

\subsection{Overview}

The criminal justice system could benefit from faster detection of Gunshot Residue (GSR) evidence, and the combination of Laser Induced Breakdown Spectroscopy (LIBS) and chemometrics can provide a solution to that need. Detection of GSR is crucial during the investigation of firearm-related crimes, but there is a large discrepancy in the time it takes to respond to the crime, and the forensic examiner's ability to process the evidence collected: the former can take minutes, while the latter can take months. The goal of this study was to develop a reliable LIBS screening approach capable of detecting GSR in just a few minutes with minimal damage to the sample, high specificity, and sensitivity. Moreover, a novel micro-sampling method was developed to gather 3-dimensional data about the simultaneous occurrence of IGSR markers from a discrete space. The method is capable of micro-spatial chemical analysis from just two laser shots fired at an area of 100um diameter. The performance of the micro-spot method is compared to a previously published bulk-line method. Superior accuracy, spatial information of IGSR distribution in the sample, and a less invasive sampling, are some of the advantages of the newly proposed method. A benefit afforded by this approach is the use of the universal hand's collection method currently used by practitioners, while leaving over $99 \%$ of the stub left unaltered for further analysis.

Machine learning algorithms were used for the classification of samples derived from shooters' hands versus non-shooters hands, based on their LIBS spectrochemical data. Four different approaches - critical threshold, Logistic Regression, Naïve Bayes, and Neural Networks -were applied to examine the performance and accuracy of two different ablation patterns (microspot and bulk-line mode). A validation set of 326 samples originated from 51 non-shooters and 56 known shooters resulted in an overall accuracy between $87-100 \%$, depending on the ablation pattern and the type of prediction model applied. The incorporation of this rapid screening and statistical decision-making approach could offer more efficient case management in firearmrelated investigations. An advantage of the machine learning classification methods is they provide probabilistic outputs that can be utilized for a more objective interpretation of the forensic evidence. 
The following chapter is an adaptation of a previously published article (C2019:

K Menking-Hoggatt, L Arroyo, J Curran and T Trejos. Novel LIBS method for chemical micromapping of inorganic gunshot reside collected from hand samples, Journal of Chemometrics, Dec 2019. https://doi.org/10.1002/cem.3208

\subsection{Introduction}

Laser Induced Breakdown Spectroscopy (LIBS) provides spectrochemical information with the additional benefit of micrometer scale resolution, which is advantageous to Gunshot Residue (GSR) identification. Finding specific elemental markers in a small area provides more discrimination for the presence or absence of GSR and is indicative of the chemical and physical changes that occur during a firing event. When a gun is fired, a series of thermodynamic and chemical processes occur, depositing inorganic and organic residues near the firing event. The forceful striking of the primer by the firing pin causes sparks, which ignite the propellant, and generate a plume of fine particles and materials containing Inorganic and Organic GSR (IGSR, and OGSR, respectively). ${ }^{1}$ The discharged plume will contain vaporized and partially molten particles, which are created at high temperatures $\left(1500-2000{ }^{\circ} \mathrm{C}\right)$, pressures $(\sim 104 \mathrm{kPa})$, and within one ten-thousandth of a second. ${ }^{2}$ The rapid heating and cooling of the vaporized materials form spherical particles due to the cohesive properties of the compounds. ${ }^{3}$ Studies have also confirmed a range of sizes and morphologies due to variations in chemical composition and the mechanical stress the particles may undergo after being deposited on a surface. ${ }^{4,5}$ Current standard practice relies on the identification of IGSR particles exhibiting distinctive elemental composition and spherical shape, typically ranging in size from 0.5-5 $\mu \mathrm{m} .{ }^{6}$ Nonetheless, the current standard acknowledges that GSR particles can be irregular, so agreement of elemental composition within the micrometer sized particles is necessary for identification.

A majority of standard ammunition utilizes the compounds lead styphnate as the initiator, barium nitrate as the oxidant, and antimony sulfide as the fuel, all contained within the primer. ${ }^{1,7,8}$ Therefore, standard IGSR elements, such as lead $(\mathrm{Pb})$, barium $(\mathrm{Ba})$, and antimony (Sb), are generated and then used to identify GSR. Some additional $\mathrm{Pb}$ can be produced by the bullet and other elements such as aluminum $(\mathrm{Al})$, copper $(\mathrm{Cu})$, and zinc $(\mathrm{Zn})$ can be contributed by the bullet and the cartridge case. ${ }^{1,9-11}$ Non-toxic ammunition manufacturers can replace the standard heavymetal compounds with other inorganic elements, such as strontium $(\mathrm{Sr})$ or potassium $(\mathrm{K})$ in the 
oxidizers, or other heavy-metal free ingredients, such as diazo dinitrophenol or calcium silicide. ${ }^{12-}$ ${ }^{14}$ Consequently, the ability of an analytical instrument like LIBS to identify multiple elements within a micrometer area is beneficial to GSR identification and interpretation.

As the current standard for IGSR analysis, Scanning Electron Microscopy-Energy Dispersive X-ray Spectroscopy (SEM-EDS) is versatile and employs multiple types of detectors well-catered to the purpose of analysis. The SEM portion scans a high-energy and finely-focused electron beam in an $\mathrm{x}-\mathrm{y}$ raster pattern to build a composite gray-scale image of the sample surface, using either secondary or backscatter electrons. ${ }^{15,16}$ This allows the morphology of the IGSR particle to be imaged. X-rays are also produced when the electron beam interacts with the sample. ${ }^{16,17}$ Characteristic X-rays can be used to identify and quantify elements in a discrete area ranging from $100 \mathrm{~nm}$ to $1 \mu \mathrm{m}$, depending on composition of the sample and beam energy. ${ }^{15}$ This allows the elemental composition of an IGSR particle to be measured. Yet, the emerging market of nontoxic ammunition will challenge the current technique since different parameters are required for automated analysis and accurate detection of lower atomic number elements. ${ }^{13}$ Regardless of its widespread use, the shortcomings of SEM-EDS are visible in the high cost of the instrument and the extensive time to complete the analysis which is typically several hours per sample. $^{18}$

Color tests have often been used for preliminary screening of suspected GSR during the firearm investigation because they offer rapid testing combined with the ease of use. ${ }^{19}$ The Griess color test produces an orange color in the presence of nitrites, which is a common OGSR byproduct of the propellant. ${ }^{2,12,20,21}$ Another frequently used color test is sodium rhodizonate, with a positive result for lead being a deep-purple color. To a lesser extent barium can be identified if a pink color is also present before the addition of the acid., ${ }^{21-23}$ While the color tests are still used to determine shooting distance, they are ineffective for detection of GSR on an individual's hands due to the lack of specificity and prevention of further confirmatory testing due to their destructive nature. ${ }^{3,12,23}$ Also, the presence of these compounds and elements are common in the environment, so a suspect or surface can test positive, yet never have handled or have been in the vicinity a firearm discharge event. Consequently, most departments and agencies have stopped using color tests for analyzing suspects hands due to high false positive rates. ${ }^{12}$

LIBS is an attractive alternative to the analysis of IGSR because it provides a solution to some of the challenges faced by current instrumentation; speed of analysis, multi-elemental 
detection, superior selectivity and sensitivity, and the application of micrometer scale spatial resolution on the surface of a standard GSR collection stub. First, a laser is focused on an area ranging from 4-200 $\mu \mathrm{m}$ in diameter. The laser-induced breakdown process occurs when a temporal plasma forms on the surface of the sample, causing vaporization and excitation of ions, atoms, and molecules as high irradiance levels are reached, typically greater than $10^{7} \mathrm{~W} \mathrm{~cm}^{-2} \cdot{ }^{24}$ The elementspecific atomic and ionic emissions are rapidly collected and dispersed by a spectrometer to obtain a spectrum representative of the sample's elemental composition. ${ }^{25}$ A detector, such as a charge coupled device (CCD) or an intensified charge coupled device (iCCD), measures the spectrometer's output signal. ${ }^{24}$

LIBS technology also offers field portability since there is no sample preparation involved, which is an attractive feature for environmental and forensic science applications. ${ }^{11,26-28}$ DoñaFernández and colleagues performed a comparison between current SEM-EDS technology and a portable LIBS using 135 samples collected from shooters, non-shooters, and the clothing of police officers. The portable LIBS instrument was found to have over $90 \%$ correct classification. $^{11}$ Additional studies have further demonstrated the potential of LIBS to simultaneously provide both multielemental and spatial information around bullet orifices on large surface areas using different ablation patterns. ${ }^{22,23,29-31}$ The results of these studies demonstrate the promise and versatility of a technique such as LIBS to strengthen the value of GSR evidence. ${ }^{1,2,11,32}$

Besides the need for rapid identification of GSR, the forensic community also faces the challenge of interpretation, which can partially be addressed through large population studies and statistical analysis. A variety of statistical approaches have been studied to enhance objectivity on decision thresholds, classification, and prediction of sample groups, ${ }^{33,34}$ while this study focused on the use of machine learning algorithms. ${ }^{35,36}$ These methods are attractive because they can handle large amounts of data and classify samples, which is useful for the wide spectral range and additional spatial information generated during LIBS analysis. Rapid evidence processing and fast decision-making are crucial to the investigation process due to the transitory nature of GSR,, $37-39$ and the inherent threat to public safety of firearm-related crimes. Our study proposes a LIBS method and subsequent classification using objective statistical modeling as a solution for rapid detection of IGSR from hands of suspects of interest. A novelty of the proposed approach is the incorporation of micro-sampling methods that allow for simultaneous confirmation of multiple IGSR markers along with micrometer scale spectrochemical spatial information. The research 
presented in this article also compares statistical methods, determines performance measures of ground-truth known samples, and demonstrates the applicability of LIBS for firearm-related investigations.

\subsection{Materials and Methods}

\subsubsection{Sample Preparation for Method Optimization}

Samples were prepared using the current GSR stub design employed by law enforcement and forensic practitioners for the collection and subsequent analysis of GSR evidence by SEMEDS. The GSR stubs consisted of $12 \mathrm{~mm}$ diameter, double-sided adhesive carbon conductive tabs (Pelco ${ }^{\mathrm{TM}}$, TedPella, CA) mounted in a double layer on a $12.7 \mathrm{~mm}$ aluminum pin mount (Zeiss specimen mounts, Al). Each stub was placed in an individual plastic storage tube (TedPella, CA). The use of these materials demonstrated viability in a previous paper study by this research group. ${ }^{32}$

An in-house IGSR particle standard was produced to simulate morphology and composition of primer residues. The standard was characterized by ICP-MS and SEM-EDS to determine the concentration of characteristic GSR elements $(\mathrm{Pb}, \mathrm{Ba}$, and $\mathrm{Sb})$ and the number of particles in a given volume. The in-house particle standard was used for optimization of the LIBS parameters in this study and contained $8.7 \pm 1.1 \mathrm{ppm}$ of $\mathrm{Pb}, 3.5 \pm 0.6 \mathrm{ppm}$ of $\mathrm{Sb}, 10.5 \pm 1.7 \mathrm{ppm}$ of $\mathrm{Ba}$, and approximately 400 particles per $1 \mathrm{uL}$.

Six stubs were spiked with a $100 \mu \mathrm{L}$ each of the in-house GSR primer suspension solution, and the spike amount covered the entire surface of the substrate. Three stubs were also spiked with acetone only and used as blanks in the experiments. The samples were prepared in a negative pressure chemical hood and allowed to dry for 20 minutes to ensure that all the acetone evaporated. The samples were then covered with the storage tube lids and stored in cardboard boxes until analysis.

\subsubsection{Firearms and Ammunition Used for the Validation Study}

Control-shooter samples were collected at the WVU indoor ballistics lab on multiple occasions using either a Springfield XD (Croatia) chambered in a $9 \mathrm{~mm}$ caliber or a Taurus .38 
special revolver. The $9 \mathrm{~mm}$ ammunition was reloaded using the same brand of brass cartridge cases, total metal jacket bullets, and smokeless powder. Small pistol primers from Remington were used for 21 sample sets and TULAMMO primers were used for 15 sample sets. The same Remington primers were also used in the reloaded .38 special ammunition, but due to the caliber differences a different type of bullet and cartridge case were used, and 20 sample sets were collected. A full list of the ammunition and firearms used is found in Table 1.

Table 1: Summary of ammunition and firearms used in the study.

\begin{tabular}{|c|c|c|c|c|c|c|}
\hline Firearm & $\begin{array}{l}\text { Type of } \\
\text { firearm }\end{array}$ & $\begin{array}{c}\text { Type of } \\
\text { Ammunition }\end{array}$ & $\begin{array}{l}\text { Bulle } \\
\text { t type }\end{array}$ & Gunpowder type & Primer type & Caliber \\
\hline $\begin{array}{c}\text { Sccy } \\
\text { CPX2 TT }\end{array}$ & pistol & specialty loaded & $\begin{array}{l}\text { Speer } \\
\text { TMJ }\end{array}$ & Winchester 231 & $\begin{array}{l}\text { Remington } 1 / 2 \text { small pistol } \\
\text { primers Kleanbore } C\end{array}$ & $9 \mathrm{~mm}$ \\
\hline $\begin{array}{c}\text { Sccy } \\
\text { CPX2 TT }\end{array}$ & pistol & specialty loaded & $\begin{array}{l}\text { Speer } \\
\text { TMJ }\end{array}$ & Winchester 231 & $\begin{array}{l}\text { TULAMMO KVB-9 small } \\
\text { pistol primers }\end{array}$ & $9 \mathrm{~mm}$ \\
\hline $\begin{array}{c}\text { Taurus } \\
.38 \\
\text { special }\end{array}$ & revolver & reloaded & $\begin{array}{l}\text { Berry' } \\
\text { s HP }\end{array}$ & Accurate \#2 powder & $\begin{array}{c}\text { Remington } 1 / 2 \text { small pistol } \\
\text { primers Kleanbore }(C)\end{array}$ & 0.38 special \\
\hline
\end{tabular}

\subsubsection{Sample Preparation and Collection of Residues from Shooter and Non-Shooter's Hands}

The same GSR stubs described in section 2.1 were used for collection of either controlshooter samples or background populations from the hands of individuals. A critical aspect of this study was to design the analysis to be compatible with the current protocol of GSR evidence collection. With this approach, a total of 51 sets of background individuals (non-shooters) were collected, along with 56 sets of control-shooter samples collected after firing a gun five times. A background set consisted of either a set of two stubs from both the left and right hand, or a single stub for only the dominant hand from individuals who had not handled a firearm within the last 24 hours. A control-shooter set consisted of four samples collected from the left palm, right palm, left back, and right back of the hands. The control-shooter samples and the background samples were collected at either the West Virginia University Indoor Ballistics Laboratory (FIS, WVU) or at the Summit Bechtel Reserve National Scout Jamboree in WV. Nitrile gloves (Fisher Scientific, NH) and a lab coat were worn during all collections. Samples were stored in a GSR stub holder, inside cardboard boxes, placed in self-sealing plastic bags and then stored in plastic storage containers. During the process of collection and analysis, routine blanks and negative controls were collected to ensure there was no cross contamination. 


\subsubsection{LIBS Experimental Setup}

A J200 Tandem Model LIBS (Applied Spectra, CA) was used for analysis of the stubs in both the optimization and validation study. The instrument has a $266 \mathrm{~nm}$ high-power, Q-switched, Neodymium doped Yttrium Aluminum Garnet (Nd YAG) laser with a 10-nanosecond pulse, a sixchannel Czerny-Turner spectrometer with a spectral range from 190 to $1040 \mathrm{~nm}$, and a CCD-based broadband detector. The prepared stubs were placed in the ablation chamber using a custom stage platform with a hole in the center that securely held the pin mount and ensured the surface remained even during analysis to maintain proper focus on the surface. All samples were analyzed in the ablation chamber one at a time and the chamber was purged for 45 seconds with $1 \mathrm{~L} / \mathrm{min}$ of argon (ultrahigh purity, Matheson, WV) before performing analysis.

Optimization was conducted on 6 stubs spiked with $100 \mathrm{uL}$ of in-house GSR standard and 3 blank stubs as negative controls. Each stub was used multiple times to collect different parameters for the Box-Behnken experimental design. This method is suited for LIBS parameter optimization due to the design's ability to analyze multiple levels and factors with a minimal number of experimental runs, and whilst still considering interactions. Also, a total of six replicates with the previously validated LIBS bulk line method ${ }^{32}$ were collected for method comparison. The following four factors at three different levels (low, medium, and high) were tested: laser frequency $(5,7.5,10 \mathrm{~Hz})$, number of shots $(1,2,3)$, spot size $(100,125,150 \mu \mathrm{m})$, and laser energy $(50 \%$, $75 \%, 100 \%$ ). The central measurement points (all parameters at medium, or middle level) were also repeated in triplicate as an estimate of error in the experimental design. An experimental run order can be found in Table 2. The optimization design was set to maximize the Signal-to-noise Ratio (SNR) of lead $(\mathrm{Pb})$, barium $(\mathrm{Ba})$, and antimony $(\mathrm{Sb})$. Negative controls were performed in triplicate using the stubs spiked with blank acetone. Once completed, the GSR stubs were stored in the stub storage containers inside cardboard boxes and in the laboratory for possible further analysis.

Optimization and validation were performed for the micro-spot and bulk line method on the same samples, and a list of the optimum parameters is shown in Table 3. The validation set consisted of samples collected from 51 background non-shooters (up to 102 subsamples) and 56 control-shooters (up to 224 subsamples). For the purpose of this study, a "sample" is defined as the collective stubs recovered from the hands of a donor. Therefore, a true positive result was recorded if any of the subsamples (i.e., any of the 4 stubs) resulted in positive identification of 
GSR markers. On the other hand, a true negative result was recorded if none of the subsamples produced a signal for more than one GSR marker above the threshold values.

Table 2: Box-Behnken experimental design for micro-spot method optimization.

\begin{tabular}{|c|c|c|c|c|c|}
\hline Run order & Pattern & $\begin{array}{l}\text { Frequency } \\
\text { (Hz) }\end{array}$ & $\begin{array}{l}\text { Spot size } \\
(\mu \mathrm{m})\end{array}$ & $\begin{array}{l}\text { Number of } \\
\text { laser shots }\end{array}$ & Laser (\%E) \\
\hline 1 & $-0+0$ & 5 & 75 & 3 & 75 \\
\hline 2 & $0--0$ & 7.5 & 50 & 1 & 75 \\
\hline 3 & $-00-$ & 5 & 75 & 2 & 50 \\
\hline 4 & $00+-$ & 7.5 & 75 & 3 & 50 \\
\hline 5 & --00 & 5 & 50 & 2 & 75 \\
\hline 7 & $+0+0$ & 10 & 75 & 3 & 75 \\
\hline 8 & +-00 & 10 & 50 & 2 & 75 \\
\hline 9 & $0+-0$ & 7.5 & 100 & 1 & 75 \\
\hline 10 & ++00 & 10 & 100 & 2 & 75 \\
\hline 11 & $+0-0$ & 10 & 75 & 1 & 75 \\
\hline 12 & $-0-0$ & 5 & 75 & 1 & 75 \\
\hline 13 & $0++0$ & 7.5 & 100 & 3 & 75 \\
\hline 14 & 0000 & 7.5 & 75 & 2 & 75 \\
\hline 15 & 0000 & 7.5 & 75 & 2 & 75 \\
\hline 16 & 0000 & 7.5 & 75 & 2 & 75 \\
\hline 17 & $00++$ & 7.5 & 75 & 3 & 100 \\
\hline 18 & $0-0-$ & 7.5 & 50 & 2 & 50 \\
\hline 19 & $0+0+$ & 7.5 & 100 & 2 & 100 \\
\hline 20 & $+00-$ & 10 & 75 & 2 & 50 \\
\hline 21 & -+00 & 5 & 100 & 2 & 75 \\
\hline 22 & $0-0+$ & 7.5 & 50 & 2 & 100 \\
\hline 23 & $+00+$ & 10 & 75 & 2 & 100 \\
\hline 24 & $0-+0$ & 7.5 & 50 & 3 & 75 \\
\hline 25 & $00--$ & 7.5 & 75 & 1 & 50 \\
\hline 26 & $00-+$ & 7.5 & 75 & 1 & 100 \\
\hline 27 & $0+0-$ & 7.5 & 100 & 2 & 50 \\
\hline 28 & $-00+$ & 5 & 75 & 2 & 100 \\
\hline
\end{tabular}


Table 3: LIBS optimized parameters used for the ablation methods validation study

\begin{tabular}{rcc}
\hline Parameter & $\begin{array}{c}\text { Bulk-Line } \\
\text { Method }\end{array}$ & $\begin{array}{c}\text { Micro-Spot } \\
\text { Method }\end{array}$ \\
\hline Spot Size $(\boldsymbol{\mu m})$ & 100 & 100 \\
\hline Gate delay $(\boldsymbol{\mu} \mathbf{)})$ & 0.5 & 0.5 \\
\hline Laser Pulse Frequency $(\mathbf{H z})$ & 10 & 5 \\
\hline Stage Velocity $(\mathbf{m m} / \mathbf{s})$ & 0.15 & 1 \\
\hline Line Length $(\mathbf{m m})$ & 7 & $\mathrm{n} / \mathrm{a}$ \\
\hline Average Laser Output Energy & 14.7 & 15.5 \\
$(\mathbf{m J})$ & & 1 \\
\hline Gas Flow Rate $(\mathbf{L} / \mathbf{m i n})$ & 1 & 2 \\
\hline Number of Shots & 469 & Yes \\
\hline Accumulate Data & Yes & 25 \\
\hline Total Spectra Collected & 1 & 91 \\
\hline Acquisition Time $(\mathbf{s e c})$ & 55 &
\end{tabular}

\subsubsection{Data Preprocessing and Statistical Analysis}

Aurora software (Applied Spectra, CA) was used to crop the collected spectrum to lie within selected spectral regions and then background subtraction, peak identification, and peak integration on the peaks of interest, were performed in that order. The integrated peak areas were imported into Excel 2016 (version 15.24, Microsoft Corporation) and combined into one workbook. SNR for Si $(251.6 \mathrm{~nm}, 288.1 \mathrm{~nm}), \mathrm{Al}(308.2 \mathrm{~nm}, 396.1 \mathrm{~nm}), \mathrm{Sb}(252.8 \mathrm{~nm}, 259.8 \mathrm{~nm})$, $\mathrm{Cu}(324.7 \mathrm{~nm}, 327.4 \mathrm{~nm}), \mathrm{Pb}(405.8 \mathrm{~nm}, 374.0 \mathrm{~nm})$, and Ba (455.4 nm, $493.3 \mathrm{~nm}, 553.4 \mathrm{~nm}, 614.1$ $\mathrm{nm}$, and $705.9 \mathrm{~nm}$ ) was calculated by using the background of the spectrum in proximity to the peak of interest. The SNR for the most intense peaks of Sb (259.8 nm), Cu (324.7 nm), Al (396.1 $\mathrm{nm}), \mathrm{Pb}(405.8 \mathrm{~nm})$, and $\mathrm{Ba}(455.4 \mathrm{~nm})$ were imported into JMP Pro 14 software (SAS, Cary, NC) and R Studio (open source, 1.2.1335) for method optimization and further statistical analysis and comparison of the two LIBS ablation patterns.

The performance rates for the bulk and micro-spot methods were evaluated using four approaches. First, an exploratory critical threshold method was used to evaluate the data and examine the variance and mean of the different groups. This was calculated in the same manner as in Trejos et. al. ${ }^{32}$ For the micro-spot method, analysis was performed on one stub from each control-shooter sample and each analysis produced 25 spectra per stub due to the $5 \times 5$ spot grid pattern. For the bulk line method, analysis was performed on four stubs per individual, but only one accumulated spectrum was produced per stub. 
Three classifier methods were used in this study to evaluate their ability to predict class membership: Logistic Regression (LR), Naïve Bayes (NB) and Neural Networks. Both LR and NB were performed using open-source R software and the statistical package caret. Two-fold cross validation was repeated ten times. This means that approximately half of the data was used for validation and the other half for testing. The training and test data sets are selected at random. Therefore, to avoid the results being swayed by a single random selection, the procedure was repeated ten times, and the average performance reported. The results of the algorithms provided confusion matrices, which were used to calculate the performance rates.

A neural network was also used as a supervised prediction method. To account for the relatively small sample size, the k-fold option divided the data into three sub-sets $(60 \%$ of the data for training, $20 \%$ for validation, and $20 \%$ for testing). The k-fold option accomplished this by processing the data 5 times with different combinations of data sets to ensure the algorithm was being properly trained. In neural networks, an activation function considers the interaction effects in the parameters and transform the values between each layer as part of the artificial learning process. After the transformation, the network decides which neuron passes the value into the next layer. Given the simple layer structure of the neural network used in this dataset (Figure 1), a nonlinear sigmoid, hyperbolic function (TanH) was applied to each node. The sigmoid TanH function is more adaptable to small differences in the data and better differentiates the output than a linear function. Other non-linear functions were also tested (Sigmoid and Rectified Linear Unit) but the TanH function performed best for this dataset. We anticipate that ReLU function may work better when the model becomes more complex, with more parameters and hidden layers. The TanH function performed a recombination of the response depending on the training data used, hence the need to run the neural network five different times to assess the training adequacy. The functions produced an output of the classification (shooter vs.

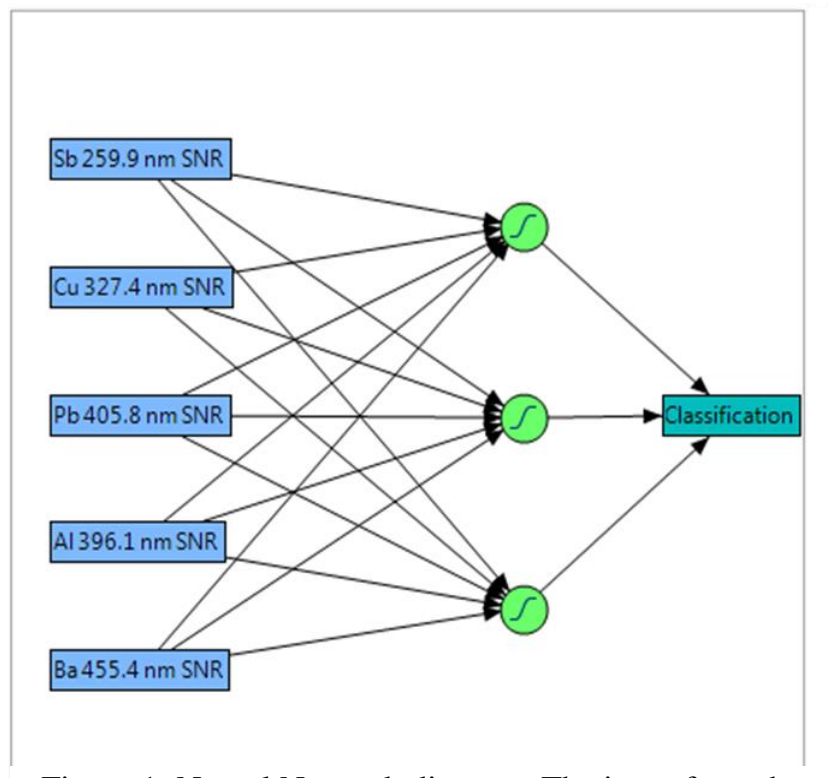

Figure 1: Neural Network diagram. The input from the five elements is processed by each node (green circles) within the single hidden layer. The output is the form classification of shooter vs non-shooter. 
non-shooter) and the results of the confusion matrices were used to calculate the misclassification rates and accuracy.

\subsection{Results and Discussion}

\subsubsection{Spot Micro-Sampling Method Optimization}

LIBS has the capability to provide multi-elemental and spatial information, which is an advantageous trait due to the complexity of GSR's elemental composition. A previous study conducted by our research group ${ }^{32}$ validated the bulk-line method, in which one accumulated spectrum was collected for a $100 \mu \mathrm{m}$ width by $7 \mathrm{~mm}$ length line pattern using 469 shots. This previous method demonstrated no less than $87 \%$ accuracy for GSR classification. Although the line method was efficient, a decision was made to exploit the $x$ and y coordinates recorded by the software in combination with finely focused laser spot size afforded by LIBS. In this study, we aimed to further push further the capabilities of LIBS by moving away from a "micro-bulk" ablation line model to a "micro-spot" model capable of producing chemical information from only 2 shot ablations in a reduced space. This mapping method allows simultaneous detection of GSR markers (i.e. $\mathrm{Pb}, \mathrm{Ba}$, and $\mathrm{Sb}$ ) on a fixed spatial area only $100 \mu \mathrm{m}$ in diameter and producing 25 individual spectra, yielding an accuracy of no less than $99.5 \%$ for the evaluated datasets.

A central aspect of GSR evidence analysis is the ability to find the characteristic elements in specific combination within a single small particle. So, from a forensic perspective, chemical micro-spot has several benefits. First, when we can narrow down the area from where signal is produced, this increases the confidence that all inorganic markers are originating from a single particle, or an isolated group of particles in a reduced area. A bulk analysis, on the other hand, could be the sum of numerous particles, or worse, the product of contaminants in the sample that mimic the IGSR composition. In either scenario, we do not have the added benefit of knowing where in the bulk-line ablation pattern a combination of the GSR markers were found simultaneously.

Second, since GSR particles are randomly distributed on the substrate during collection, micro-spot allows for a more comprehensive analysis on the surface while still maintaining the integrity of the sample. The 25 ablation spots results in a smaller amount of the stub being ablated - about $0.2 \%$ as opposed to the bulk method at about $0.6 \%$ - and causes less damage to 
the surface of the stub since the grid pattern only requires two laser shots per spot versus the 469 shots of the bulk method. Therefore, this study assessed if micro-spot LIBS method is fit for purpose and produces reliable results similar to or superior to the bulk line ablation.

Both methods can provide the multielement information, but only one spectrum is obtained for the entire line pattern. Although LIBS cannot ablate a single GSR particle, the micro-spot method allows for spatial resolution by combining the spectrum collected with a $100 \mu \mathrm{m}$ diameter area paired with its $x, y$, and $z$ coordinates on the surface of the GSR stub. The grid pattern developed contains 25 areas of analysis, generating an individual spectrum with the respective spatial coordinates and spectral information for multiple elements and emission lines, therefore increasing confidence in the results with multiple spectra to analyze. A visual comparison of the two methods can be found in Figure 2. For illustration purpose, only one of the spots is highlighted in the Figure 2 (spot 13), but each spot has a similar set of associated spectra spanning a spectral range of wavelengths from 190-1054 nm.

The same type of carbon adhesive stubs in this study were used in our previous study and demonstrated that the substrate did not create background interference for LIBS signals. ${ }^{32}$ Optimization of the laser parameters was conducted by a surface response experimental design. The method was then validated and compared to the micro-bulk method using datasets collected from the hands of shooters and non-shooters.

A Box Behnken response surface design was utilized on the micro-spot method to determine the optimal ablation and detection parameters for maximum signal-to-noise as discussed in section 2.4. The $\mathrm{SNR}$ of $\mathrm{Pb}, \mathrm{Ba}$, and $\mathrm{Sb}$ at major emission lines - atomic (I) line at $405.8 \mathrm{~nm}$, ionic (II) line at $455.4 \mathrm{~nm}$, and atomic (I) line at $259.8 \mathrm{~nm}$, respectively-was input into in the response surface design to maximize signal-to-noise ratio of these elements. Additional emission lines were monitored to confirm the presence of these elements of interest; $\mathrm{Ba} 493.3 \mathrm{~nm}$ (I), Ba 553.4 nm (I), Ba $614.1 \mathrm{~nm}$ (II), Ba 705.9 nm (I), Pb 368.3 nm (I), and Sb 252.8 nm (I). Other elements often associated with GSR were monitored, such as Al, Ca, Zn, Ti, Sn, Sr, and Cu. The method's design incorporated detection of numerous elements other than the standard $\mathrm{Pb}, \mathrm{Ba}$, and $\mathrm{Sb}$, as is one of the many benefits of using LIBS instrumentation. Also, the ability to detect these other elements will be beneficial in the future analysis of nontoxic IGSR.

The respective contour maps and critical values obtained from the optimization experiment allowed the determination of the ideal parameters for the micro-spot method. Parameters were 
chosen that resulted in the highest increase of the SNR for both $\mathrm{Pb}$ and $\mathrm{Ba}$. When the data is a good fit for the Box-Behnken Design, then the mean square of lack of fit should reflect only the random error in the model and not be statistically different from the mean square of the pure error; in other words the two errors should be comparable. ${ }^{40}$ The $p$-value for the design was greater than the alpha level of 0.05 and indicated the difference between the model data and the experimental data was not significantly different. This provided insight into which independent variables had the greatest influence on the signal-to-noise ratio of the elements measured. The chosen parameters were carried onto the validation step and a comparison of the parameter for two methods can be found in Table 3.

The micro-spot method's area of analysis is confined to a space of $1 \mathrm{~mm}^{2}$ and only uses two laser shots per spot analyzed, causing very little ablation of the adhesive. The ablation interaction provides minimally damage that is only visible at high magnification, such as SEM images, and leaves over $99.8 \%$ of the stub unablated for any further analysis. The spatial information of the spots collected, and the individual spectra, assist to increase the certainty in the presence of the elements of interest within a micrometer sized area.

\subsubsection{Performance Comparison of Ablation Methods and Classification Approach}

The performance of the micro-spot method was compared to the bulk-line method on samples from both controlled background (non-shooters) and shooter samples. The size of the sampling substrate allowed for both methods to be performed on the same stub for direct comparison. Performance rates of the respective statitical methods are summarized in Table 4.

A critical threshold method was used as an exploratory technique to examine the overall performance of the line versus micro-spot method. A result was considered positive when a signalto-noise ratio was three times higher than the standard deviation of the background mean for each element of interest, while a negative result was determined when the signal-to-noise ratio was below the threshold. Calculating this critical threshold value allowed for an understanding of the mean signal-to-noise ratio and variation observed on the true positives and true negative sets. The mean and standard deviation also allowed the calculation of the samples set power, which was over $95 \%$ for both methods.

The micro-spot ablation method consistently provided superior performance than the bulkline method as observed in the performance rates listed in Table 4. Moreover, the micro-spot 
method provided spatial information not available in the bulk-line method; increase in the number of spectra collected and less destruction of the sample substrate's background. Obtaining 25 spectra with spatial information increases the chances to detect GSR since each spot can be analyzed separately for the identification of characteristic GSR markers. Accumulation of the signal is still performed in the spot mapping method, but only 2 shots per area. This improved the SNR because when the laser ablates a GSR particle(s) — single or multiple particles can exist within the $100 \mu \mathrm{m}$ spot size - there is less dilution of the signal from the substrate. On the other hand, in the line method, the accumulation of spectra is done throughout the 469 shots. If only a percent of those shots hit GSR particles, the overall signal is diminished by background noise. As a result, the micro-spot method allowed a more efficient sampling than the bulk-line method.

Table 4: Comparison of the statistical analysis and performance measures for validation study.

\begin{tabular}{|c|c|c|c|c|c|c|c|c|}
\hline \multirow[b]{2}{*}{$\begin{array}{r}\text { Performance } \\
\text { measure }\end{array}$} & \multicolumn{2}{|c|}{ Critical Threshold } & \multicolumn{2}{|c|}{$\begin{array}{c}\text { Logistic } \\
\text { Regression }\end{array}$} & \multicolumn{2}{|c|}{ Naïve Bayes } & \multicolumn{2}{|c|}{ Neural Networks } \\
\hline & $\begin{array}{l}\text { Bulk- } \\
\text { line }\end{array}$ & $\begin{array}{l}\text { Micro- } \\
\text { spot }\end{array}$ & $\begin{array}{l}\text { Bulk- } \\
\text { line }\end{array}$ & $\begin{array}{l}\text { Micro- } \\
\text { spot }\end{array}$ & $\begin{array}{l}\text { Bulk- } \\
\text { line }\end{array}$ & $\begin{array}{l}\text { Micro- } \\
\text { spot }\end{array}$ & $\begin{array}{c}\text { Bulk- } \\
\text { line }\end{array}$ & Micro-spot \\
\hline False positive & $0.0 \%$ & $0.0 \%$ & $5.5 \%$ & $0.3 \%$ & $11.5 \%$ & $0.2 \%$ & $0.0 \%$ & $0.0 \%$ \\
\hline False negative & $27.0 \%$ & $0.0 \%$ & $2.7 \%$ & $0.3 \%$ & $3.6 \%$ & $0.9 \%$ & $0.0 \%$ & $0.0 \%$ \\
\hline $\begin{array}{r}\text { True negative } \\
\text { (Specificity) }\end{array}$ & $100.0 \%$ & $100.0 \%$ & $94.5 \%$ & $99.7 \%$ & $88.5 \%$ & $99.8 \%$ & $100.0 \%$ & $100.0 \%$ \\
\hline $\begin{array}{r}\text { True positive } \\
\text { (Sensitivity) }\end{array}$ & $73.0 \%$ & $100.0 \%$ & $97.3 \%$ & $99.7 \%$ & $96.4 \%$ & $99.1 \%$ & $100.0 \%$ & $100.0 \%$ \\
\hline Accuracy & $87.0 \%$ & $100.0 \%$ & $96.4 \%$ & $99.7 \%$ & $93.7 \%$ & $99.5 \%$ & $100.0 \%$ & $100.0 \%$ \\
\hline
\end{tabular}

In this particular application, we investigated the ability of machine learning classifiers to distinguish between two groups, which we named here non-shooters (samples from background individuals) and shooters (samples from individuals in the vicinity of a firing). In this regard, we are interested in a categorical response that has only two possible outcomes, labeled with values of zero (non-shooters group) and one (shooters group). The independent variables used as data input were the signal-to-noise ratios of five elements associated with GSR ( $\mathrm{Sb}, \mathrm{Pb}, \mathrm{Ba}, \mathrm{Cu}$ and Al). A wide variety of classification algorithms exist in the literature. Given the number of LIBS' independent variables used here to identify gunshot residue markers, the number of samples in our 
dataset, and the dichotomous nature of the dependent variable, we have selected three supervised learning statistical methods to evaluate the performance of the LIBS methods: Binary Logistic Regression, Naïve Bayes classifier, and Neural Networks.

Logistic regression is a classification method appropriate for binary outcomes that searches for the optimal decision boundary that best separates the classes by learning the probability of a sample belonging to a given group. The algorithm focuses on maximizing the likelihood of observing the sample values and adjusts the linear separation based on the observation. In logistic regression, the predictions are transformed using the logistic function that models the probability of class membership and transforms it into a binary value (0 or 1$)$. Among the strengths of logistic regression is that the method is widely known, and the parameter's estimates are easy to interpret in probabilistic terms. The results describe the relationship between a dependent binary variable (e.g., shooter) and the independent variables ${ }^{41}$ (e.g., chemical profile of GSR markers represented as elements of interest) and a confusion matrix is generated. Using this approach, the accuracy of the micro-bulk line data was $96.4 \%$ and the micro- spot data was $99.7 \%$ (Table 4)

Naïve Bayes uses the Bayes' theorem in the classifier's decision rule assuming independence between variables (Equation 1). Given a set of variables, $\mathrm{X}=\left\{x_{1}, x_{2}, \ldots, x_{n}\right\}$, the algorithm can be expressed as:

\section{Equation 1: Formula for the Naïve Bayes Formula.}

$\operatorname{Pr}\left(y_{i}=y \mid x_{1}, x_{2}, \ldots, x_{n}\right)=\frac{\prod_{j=1}^{n} \operatorname{Pr}\left(x_{j} \mid y_{i}=y\right) \operatorname{Pr}(y)}{\prod_{j=1}^{n} \operatorname{Pr}\left(x_{j} \mid y_{i}=y\right) \operatorname{Pr}(y)+\prod_{j=1}^{n} \operatorname{Pr}\left(x_{j} \mid y_{i}=\bar{y}\right) \operatorname{Pr}(\bar{y})}$

where $y=\in\{$ shooter, non - shooter $\}$

Where the $x$ variables represent the features, or signal-to-noise ratio of the five elements, and y is the dependent variable, or class, and in our case with only two outcomes (non-shooter or shooter). As a result, Naïve Bayes derives the probability of the given feature vector being associated with a class.

One disadvantage of Naïve Bayes is that the assumption that the predictor variables are independent is not always met. However, this assumption is still practical because it reduces multidimensional tasks, simplifies the classification, and permits the class conditional densities to be calculated separately for each variable. Moreover, in this dataset correlation among variables does not seem to affect the posterior probabilities of class membership significantly. An advantage 
of Naïve Bayes is that the size of training data to estimate the classification parameters can be relatively small, and the predictions are scalable. ${ }^{42}$ From the confusion matrix generated, the performance measures were calculated (Table 4) rendering accuracy of $93.7 \%$ for the bulk-line and $99.5 \%$ for the micro-spot method, respectively.

The third machine learning method tested in this study was a neural network, another type of supervised classification algorithm. Neural Networks are models inspired by the structure of biologic neural networks. They consist of highly interconnected nodes, and the connection between these nodes is used to predict an output, or classification. It can adapt to small differences in training data sets, providing the benefit of more accurate group classifications due to neural network's adaptability and training capacity. Since there are only five elements currently being monitored, the neural network is simple, consisting of only five input variables, and three nodes within one hidden layer. A diagram of the neural network can be found in Figure 1. The preliminary results for assessment of the model were promising with both ablation methods providing $100 \%$ correct classification of the samples into shooter versus non-shooter groups.

An advantage of Neural Network analysis is their ability to learn more complex, functions, and use a combination of both linear and non-linear functions. Neural networks can be adapted quickly for more complex data sets simply by increasing the number of hidden layers or nodes, and also applying different functions to each layer to accommodate larger data sets and provide more class predictions. Therefore, the ability of neural networks to handle large, multivariate data sets make this algorithm appealing as the number of elements and classification groups are expanded in our future datasets. Another advantage of a neural network is that the algorithm does not make assumptions about normality, linearity, or variable independence when modeling the data. One disadvantage of Neural Networks is the understanding of how the prediction works, tends to not be as straightforward as the coefficients or odds ratios given in regression models, which complicates interpretation of the model and makes it difficult to explain. The lack of interpretability of individual predictors may be perceived as a limitation within the forensic context, as it does not provide enough transparency of the data presented in court, even though neural network computing is widely accepted for other purposes.

Although all the algorithms mentioned above are used to predict if a sample belongs to a particular class, their assumptions and learning mechanism are slightly different. For instance, logistic regression is a discriminative model that directly models the posterior probability by 
minimizing the error. Naive Bayes is generative and models the joint distribution of the feature and output and then predicts the posterior probability. In other words, logistic regression directly estimates the parameters of $\mathrm{P}(\mathrm{Y} \mid \mathrm{X})$, whereas Naive Bayes directly estimates parameters for $\mathrm{P}(\mathrm{Y})$ and $\mathrm{P}(\mathrm{X} \mid \mathrm{Y})$, where $\mathrm{X}$ is the variables and $\mathrm{Y}$ is the dependent variable or binary classification.

Neural networks are somewhat related to logistic regression. Indeed, logistic sigmoid functions are often used as activation functions in the hidden layer of a neural network, and therefore each layer of a neural network can be thought of as logistic regression. Neural Network is also a discriminative model but estimates many more parameters and permutations of parameters than the logistic regression, allowing for the discovery of more complex functions. Although both Logistic Regression and Naïve Bayes assumes that the input features are independent, this assumption is not as relevant in Logistic Regression as it can be accounted for in the loss function. Neural networks, on the other hand, do not make assumptions of feature independence and model the data based on the training set.

The results of this study indicate that all predictive models provide an accurate classification of samples derived from shooters' hands versus non-shooters hands, based on the LIBS elemental profiles. As expected, Logistic Regression, Naïve Bayes, and Neural Networks outperformed the critical threshold method increasing the accuracy from $87 \%$ to $100 \%$, depending on the ablation mode and classifier. Regardless of minor differences in the misclassification rates and overall accuracy, all classifiers consistently revealed a superior performance of the micro-spot over the micro-bulk line ablation mode. (Table 4) 


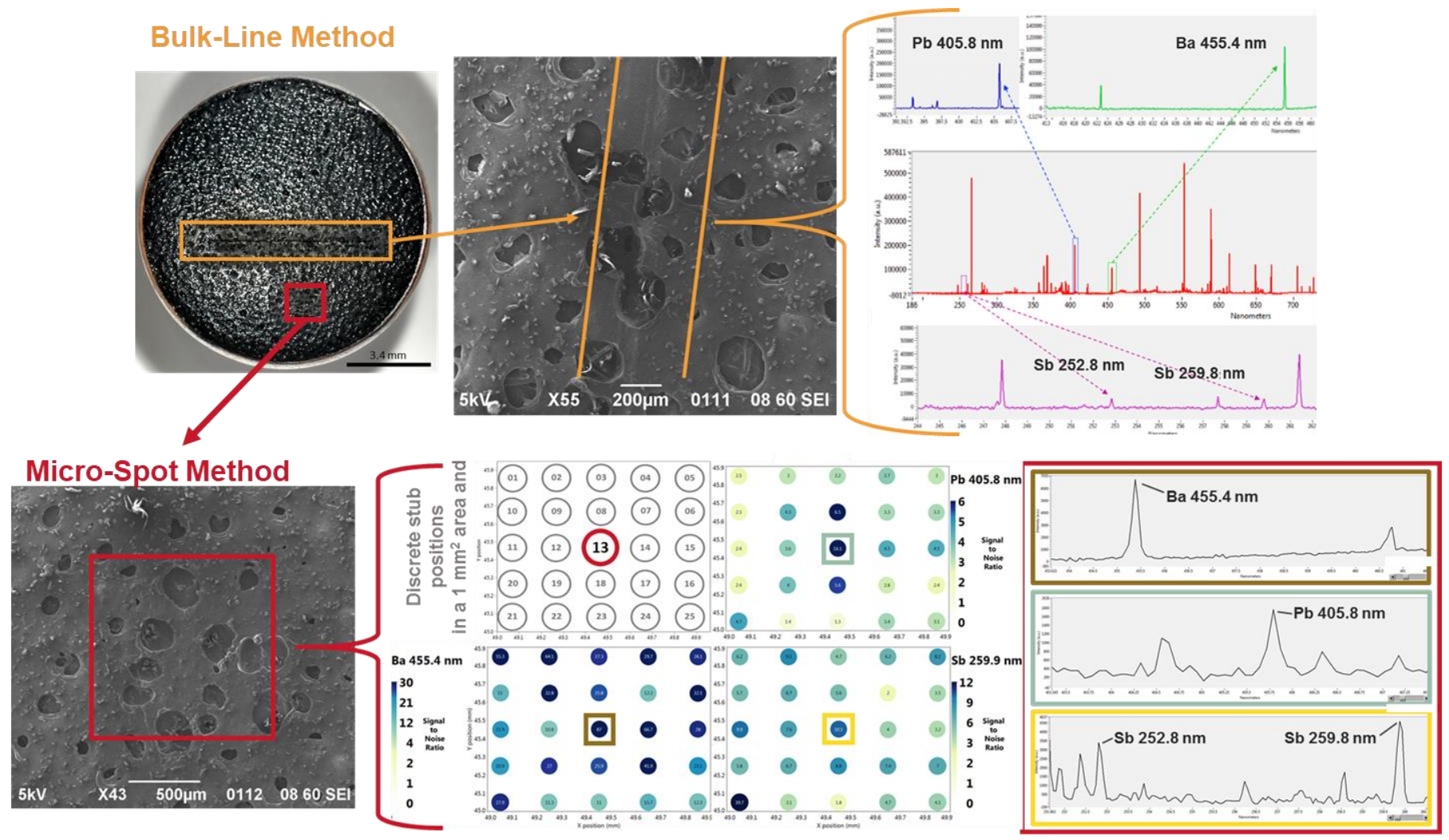

Figure 2: Comparison of the pattern and spectral information for the two ablation methods. The line method only produces one accumulated spectrum for the $7 \mathrm{~mm}$ by $100 \mathrm{um}$ line pattern. The spot method produces one spectrum for each $100 \mathrm{um}$ in diameter spot result in 25 spectra per pattern. The darker blue the square in the heat map, the higher signal-to-noise ratio (SNR) for that element. 


\subsection{Conclusion}

The results of this study demonstrated the performance of LIBS as a rapid and reliable technique for IGSR detection and the application of statistical models for the prediction of shooting activity. The study further demonstrated the usefulness of adding spatial information to confirm the simultaneous occurrence of IGSR markers in a confined area of the sample, adding more confidence in the results. The performance measures had high accuracy ranging from 87 to $100 \%$ for all the techniques tested.

The micro-spot method consistently outperformed the previously reported bulk-line method in many different aspects: the performance measures were superior, data collection increased from one spectrum to twenty-five spectra per sample, and multielement spectrochemical IGSR markers can be isolated to discrete locations on the GSR stub. Also, more accurate classification of the samples into non-shooter versus shooter groups occurred when spatial and spectral data were incorporated into the analysis processes.

In our research, Logistic Regression, Naïve Bayes, and Neural Network models showed a similar predictive ability to distinguish between shooter and non-shooter classes, and they were relatively easy to build. Although performance rates were slightly in favor of the neural network, more straightforward Logistic Regression and Naïve Bayes methods demonstrated a fit for purpose method for the interpretation of gunshot residues LIBS data. One advantage of the tested methods is that they provide probabilistic outputs that can be utilized for further interpretation of the weight of the evidence.

On-going efforts of our research group include increasing the number of samples and testing different types of backgrounds and ammunition. For example, additional populations currently under investigation include individuals with hobbies or professions that produce false positives and more modern ammunition that may not contain the typical heavy-metal GSR markers. These results of this study support a solid foundation for the expansion of future research and the ability of LIBS to provide rapid, accurate analysis of IGSR. The ability to use machine learning algorithms to calculate performance measures for the test increases the scientific validity of analysis in crime laboratories. Also, the probabilities obtained from the statistical analysis are anticipated to assist with the interpretation and presentation of evidence in the courtroom. 


\subsection{References}

(1) Dockery, C. R.; Goode, S. R. Laser-Induced Breakdown Spectroscopy for the Detection of Gunshot Residues on the Hands of a Shooter. Appl. Opt. 2003, 42 (30), 6153-6158. https://doi.org/10.1364/ao.42.006153.

(2) Silva, M. J.; Cortez, J.; Pasquini, C.; Honorato, R. S.; Paima, A. P. S.; Pimentel, M. F.; Paim, A. P. S.; Pimentel, M. F. Gunshot Residues: Screening Analysis by Laser-Induced Breakdown Spectroscopy. J. Braz. Chem. Soc. 2009, 20 (10), 1887-1894. https://doi.org/10.1590/S0103-50532009001000017.

(3) Hannigan, T. J.; McDermott, S. D.; Greaney, C. M.; O’Shaughnessy, J.; O’Brien, C. M. Evaluation of Gunshot Residue (GSR) Evidence: Surveys of Prevalence of GSR on Clothing and Frequency of Residue Types. Forensic Sci. Int. 2015, 257, 177-181.

(4) Basu, S. Formation of Gunshot Residues. J. Forensic Sci. 1982, 27 (1), 72-91. https://doi.org/10.1520/jfs11453j.

(5) Kara, I.; Sarikavak, Y.; Lisesivdin, S. B.; Kasap, M. Evaluation of Morphological and Chemical Differences of Gunshot Residues in Different Ammunitions Using SEM/EDS Technique. Environ. Forensics 2016, 17 (1), 68-79. https://doi.org/10.1080/15275922.2015.1133729.

(6) Standard Guide for Gunshot Residue Analysis by Scanning Electron Microscopy / Energy Dispersive X-Ray Spectrometry 1; West Conshohocken, PA, 2009. https://doi.org/10.1520/E1588-20.2.

(7) Mason, K.; Wuhrer, R. Detection and Characterization of Heavy-Metal-Free (HMF) Gunshot Residues Using CL, EDS and Raman Together with XRD. Mircoscopic Microanal. 2018, 24 (1), 1174-1175. https://doi.org/10.1017/S1431927618006359.

(8) Fambro, L. A.; Miller, E. T.; Vanderbos, D. D. Characterization of Lead-Free Gunshot Residue Analogs. Anal. Methods 2016, 8, 3132-3139. https://doi.org/10.1039/c6ay00725b.

(9) Maitre, M.; Kirkbride, K. P.; Horder, M.; Roux, C.; Beavis, A. Current Perspectives in the Interpretation of Gunshot Residues in Forensic Science: A Review. Forensic Sci. Int. 
2017, 270, 1-11.

(10) Hogg, S. R.; Hunter, B. C.; Smith, R. W. Elemental Characterization and Discrimination of Nontoxic Ammunition Using Scanning Electron Microscopy with Energy Dispersive X-Ray Analysis and Principal Components Analysis. J. Forensic Sci. 2016, 61 (1), 35-42. https://doi.org/10.1111/1556-4029.12881.

(11) Dona-Fernandez, A.; de Andres-Gimeno, I.; Santiago-Toribio, P.; Valtuille-Fernandez, E.; Aller-Sanchez, F.; Heras-Gonzalez, A. Real-Time Detection of GSR Particles from Crime Scene: A Comparative Study of SEM/EDX and Portable LIBS System. Forensic Sci. Int. 2018, 292, 167-175. https://doi.org/10.1016/j.forsciint.2018.09.021.

(12) Dalby, O.; Butler, D.; Birkett, J. W. Analysis of Gunshot Residue and Associated Materials: A Review. J. Forensic Sci. 2010, 55 (4), 924-943. https://doi.org/10.1111/j.1556-4029.2010.01370.x.

(13) Brozek-Mucha, Z. Trends in Analysis of Gunshot Residue for Forensic Purposes. Anal. Bioanal. Chem. 2017, 409, 5803-5811.

(14) Abrego, Z.; Grijalba, N.; Unceta, N.; Maguregui, M.; Sanchez, A.; Fernandez-Isla, A.; Goicolea, M. A.; Barrio, R. J. A Novel Method for the Identification of Inorganic and Organic Gunshot Residue Particles of Lead-Free Ammunitions from the Hands of Shooters Using Scanning Laser Ablation-ICPMS and Raman Micro-Spectroscopy. Analyst 2014, 139 (23), 6232-6241. https://doi.org/10.1039/c4an01051e.

(15) Goldstein, J.; Newbury, D. E.; Michael, J. R.; Ritchie, N. W. M.; Scott, J. H. J.; Joy, D. C. Scanning Electron Microscopy and X-Ray Microanalysis. Fourth edi. Springer: New York, NY 2018. https://doi.org/10.1007/978-1-4939-6676-9.

(16) Ul-Hamid, A. A Beginners' Guide to Scanning Electron Microscopy. Springer: Cham, Switzerland 2018. https://doi.org/10.1007/978-3-319-98482-7.

(17) Rakovan, J. A Word to the Wise: Energy Dispersive Spectrometry (EDS). Rocks Miner. 2004, 79 (3), 194-195.

(18) Castellanos, A.; Bell, S.; Fernandez-Lima, F. Characterization of Firearm Discharge Residues Recovered from Skin Swabs Using Sub-Micrometric Mass Spectrometry 
Imaging. Anal. Methods 2016, 8 (21), 4300-4305. https://doi.org/10.1039/c6ay00096g.

(19) Andrasko, J.; Maehly, A. C. Detection of Gunshot Residues on Hands by Scanning Electron Microscopy. J. Forensic Sci. 1977, 22 (2), 279-287.

(20) Brozek-Mucha, Z. Distribution and Properties of Gunshot Residue Originating from a Luger 9 Mm Ammunition in the Vicinity of the Shooting Gun. Forensic Sci. Int. 2009, 183 (1-3), 33-44. https://doi.org/10.1016/j.forsciint.2008.10.010.

(21) Chang, K. H.; Jayaprakash, P. T.; Yew, C. H.; Abdullah, A. F. L. Gunshot Residue Analysis and It Evidential Values: A Review. Aust. J. Forensic Sci. 2013, 45 (1), 3-23. https://doi.org/10.1080/00450618.2012.691546.

(22) Lopez-Lopez, M.; Alvarez-Llamas, C.; Pisonero, J.; Garcia-Ruiz, C.; Bordel, N. An Exploratory Study of the Potential of LIBS for Visualizing Gunshot Residue Patterns. Forensic Sci. Int. 2017, 273, 124-131. https://doi.org/10.1016/j.forsciint.2017.02.012.

(23) Pyl, C. Vander; Ovide, O.; Ho, M.; Yuksel, B.; Trejos, T. Spectrochemical Mapping Using Laser Induced Breakdown Spectroscopy as a More Objective Approach to Shooting Distance Determination. Spectrochim. Acta Part B At. Spectrosc. 2019, 152, 93-101. https://doi.org/https://doi.org/10.1016/j.sab.2018.12.010.

(24) Musazzi, S.; Perini, U. Laser-Induced Breakdown Spectroscopy : Theory and Applications. Springer: Berlin, Heidelberg 2014. https://doi.org/10.1007/978-3-64245085-3.

(25) Noll, R. Laser-Induced Breakdown Spectroscopy: Fundamentals and Applications; Springer-Verlag Berlin Heidelberg: Heidelberg ;, 2012. https://doi.org/10.1007/978-3-64220668-9.

(26) Rakovský, J.; Čermák, P.; Musset, O.; Veis, P. A Review of the Development of Portable Laser Induced Breakdown Spectroscopy and Its Applications. At. Spectrosc. 2014, 101 (C), 269-287.

(27) Yang, P.; Zhou, R.; Zhang, W.; Yi, R.; Tang, S.; Guo, L.; Hao, Z.; Li, X.; Lu, Y.; Zeng, X. High-Sensitivity Determination of Cadmium and Lead in Rice Using Laser-Induced Breakdown Spectroscopy. Food Chem. 2019, 272, 323-328. 
https://doi.org/https://doi.org/10.1016/j.foodchem.2018.07.214.

(28) Motto-Ros, V.; Syvilay, D.; Bassel, L.; Negre, E.; Trichard, F.; Pelascini, F.; El Haddad, J.; Harhira, A.; Moncayo, S.; Picard, J.; Devismes, D.; Bousquet, B. Critical Aspects of Data Analysis for Quantification in Laser-Induced Breakdown Spectroscopy. Spectrochim. Acta Part B-Atomic Spectrosc. 2018, 140, 54-64. https://doi.org/10.1016/j.sab.2017.12.004.

(29) Martin, M. Z.; Labbe, N.; Andre, N.; Harris, R.; Ebinger, M.; Wullschleger, S. D.; Vass, A. A. High Resolution Applications of Laser-Induced Breakdown Spectroscopy for Environmental and Forensic Applications. Spectrochim. Acta Part B-Atomic Spectrosc. 2007, 62 (12), 1426-1432. https://doi.org/10.1016/j.sab.2007.10.046.

(30) Fambro, L. A.; Vandenbos, D. D.; Rosenberg, M. B.; Dockery, C. R. Laser-Induced Breakdown Spectroscopy for the Rapid Characterization of Lead-Free Gunshot Residues. Appl. Spectrosc. 2017, 71 (4), 699-708. https://doi.org/10.1177/0003702816689099.

(31) Tarifa, A.; Almirall, J. R. Fast Detection and Characterization of Organic and Inorganic Gunshot Residues on the Hands of Suspects by CMV-GC-MS and LIBS. Sci. Justice 2015, 55 (3), 168-175. https://doi.org/10.1016/j.scijus.2015.02.003.

(32) Trejos, T.; Vander Pyl, C.; Menking-Hoggatt, K.; Alvarado, A. L.; Arroyo, L. E. Fast Identification of Inorganic and Organic Gunshot Residues by LIBS and Electrochemical Methods . Forensic Chem. 2018, 8, 146-156. https://doi.org/doi.org/10.1016/j.forc.2018.02.006.

(33) El Haddad, J.; Canioni, L.; Bousquet, B. Good Practices in LIBS Analysis: Review and Advices. Spectrochim. Acta Part B-Atomic Spectrosc. 2014, 101, 171-182. https://doi.org/10.1016/j.sab.2014.08.039.

(34) Yañez, J.; Paz Farías, M.; Zúñiga, V.; Soto, C.; Contreras, D.; Pereira, E.; Mansilla, H. D.; Saavedra, R.; Castillo, R.; Sáez, P. Differentiation of Two Main Ammunition Brands in Chile by Regularized Discriminant Analysis (RDA) of Metals in Gunshot Residues. Microchem. J. 2012, 101, 43-48. https://doi.org/https://doi.org/10.1016/j.microc.2011.10.003. 
(35) Bell, S.; Seitzinger, L. From Binary Presumptive Assays to Probabilistic Assessments: Differentiation of Shooters From Non-Shooters Using IMS, OGSR, Neural Networks, and Likelihood Ratios. Forensic Sci. Int. 2016, 263, 176-185.

(36) Gallidabino, M.; Weyermann, C.; Romolo, F. S.; Taroni, F. Estimating the Time since Discharge of Spent Cartridges: A Logical Approach for Interpreting the Evidence. Sci. Justice 2013, 53 (1), 41-48. https://doi.org/10.1016/j.scijus.2011.12.004.

(37) French, J.; Morgan, R. An Experimental Investigation of the Indirect Transfer and Deposition of Gunshot Residue: Further Studies Carried out with SEM-EDX Analysis. Forensic Sci. Int. 2015, 247, 14-17. https://doi.org/10.1016/j.forsciint.2014.10.023.

(38) Arndt, J.; Bell, S.; Crookshanks, L.; Lovejoy, M.; Oleska, C.; Tulley, T.; Wolfe, D. Preliminary Evaluation of the Persistence of Organic Gunshot Residue. Forensic Sci. Int. 2012, 222 (1-3), 137-145. https://doi.org/10.1016/j.forsciint.2012.05.011.

(39) Maitre, M.; Horder, M.; Kirkbride, K. P.; Gassner, A.-L.; Weyermann, C.; Roux, C.; Beavis, A. A Forensic Investigation on the Persistence of Organic Gunshot Residues. Forensic Sci. Int. 2018, 292, 1-10. https://doi.org/10.1016/j.forsciint.2018.08.036.

(40) Costa, V. C.; Nascimento Guedes, W.; de Santana Santos, A.; Moreira Nascimento, M. Multivariate Optimization for the Development of a Fast and Simple Ultrasound-Assisted Extraction Procedure for Multielemental Determination in Tea Leaves by Inductively Coupled Plasma Optical Emission Spectrometry (ICP OES). Food Anal. Methods 2018, 11 (7), 2004-2012. https://doi.org/10.1007/s12161-018-1171-8.

(41) Mohri, M.; Rostamizadeh, A.; Talwalkar, A. Foundations of Machine Learning. MIT Press: Cambridge, MA 2012.

Bali, R.; Sarkar, D.; Lantz, B.; Lesmeister, C. R : Unleash Machine Learning Techniques : Find out How to Build Smarter Machine Learning Systems with R : Follow This Three Module Course to Become a More Fluent Machine Learning Practitioner : A Course in Three Modules. Packt Publishing: Birmingham, UK 2016. 


\section{Section 6}

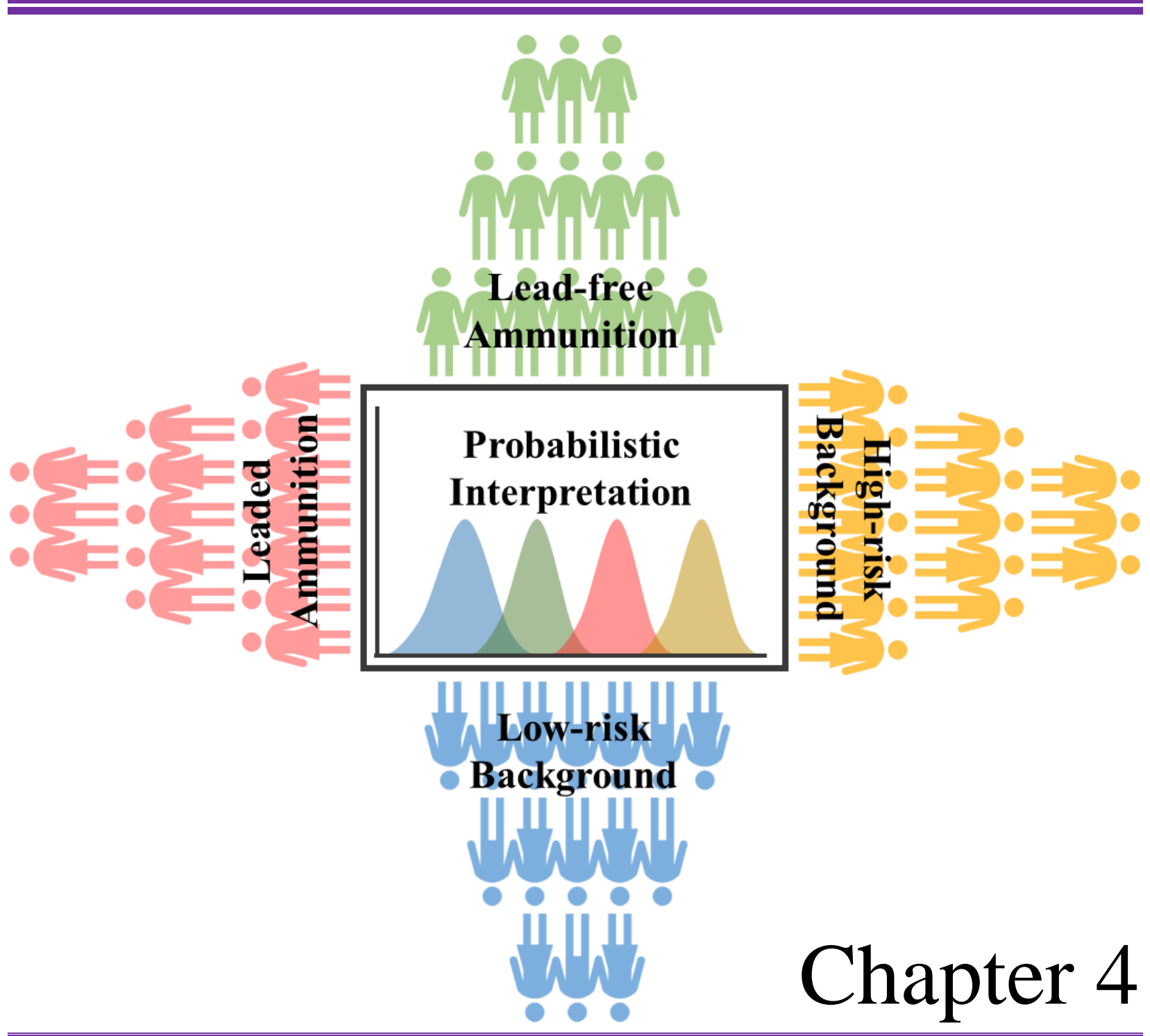

Gunshot Residue Population Study of Hands 


\section{Chapter 4: Gunshot Residue Population Study of Hands}

\subsection{Overview}

The field of GSR analysis faces many challenges, from the time it takes to complete the analysis to interpretation of the evidence. Time constraints of current methods makes a large population study difficult to perform, which is a critical factor for the statistical interpretation of the data. In response to this challenge, a rapid, micro-spatial LIBS method was applied to a large dataset consisting of residues collected from hands from over 900 individuals (>2,900 samples) from various populations. The samples were grouped into five major sets. The groups included two background populations of non-shooter individuals, one from those with at a low-risk for having GSR-like residues on their hands, and those who may pose a high-risk of GSR-like residues due to their profession or hobbies. Also, three known-shooter populations consisted of samples collected after firing only leaded, only lead-free, or a mixture of both type of ammunition. All the samples were characterized by LIBS, then a smaller subset was confirmed by the current standard for IGSR analysis using SEM-EDS. Finally, the collected data set was analyzed using multiple classification techniques and machine learning algorithms to calculate performance and error rates, with overall accuracies of $90 \%$ or higher. In addition, the probabilities generated by the machine learning algorithms were used to construct probability density functions and likelihood ratios to assist in a probabilistic interpretation of the collected data. The study conducted anticipates a beneficial impact on the forensic science community by providing a deeper understanding of GSR elements in the general background population as well as residues from the discharge of modern ammunition, and a more objective approach to the interpretation and presentation of evidence in court.

This chapter is part of a larger study in preparation for publication. The anticipated submission of the full article is summer of 2021 and will include electrochemical characterization in combination to the LIBS and SEM-EDS findings discussed here. 


\subsection{Introduction}

Gun violence is a global issue affecting public health. In the United States, the alarming rise in the number of deaths by homicide, suicide, and mass shooting is becoming a pandemic. Improvement of approaches from prevention, prediction, and policy is necessary to address this growing crisis. From law enforcement and criminal justice perspective, technological advances to achieve faster responses and more efficient evidence interpretation can significantly contribute to the fighting for safer communities.

Gunshot residue (GSR) is a commonly submitted form of trace evidence in crime laboratories, as it can provide valuable information about possible links between an individual of interest and a firearm-related event. ${ }^{1,2}$ One of the goals of these types of examinations is first to assess the uncertainty of classifying the recovered traces as GSR residue rather than residues originating from other environmental sources. Second, the examiner should evaluate the significance of the findings, considering how the traces could have been transferred and persisted given the contextual circumstances of the case. For instance, the relevance of the evidence has to be evaluated to assess the possibility of the residues being produced by a direct transfer from the firearm discharge as opposed to secondary or tertiary transferred mechanisms. Finally, these findings should be communicated to the trier of fact in a clear manner to assist in the proper interpretation of the weight of the evidence.

Different approaches have been proposed to interpret GSR evidence; some are limited to addressing questions at the source level, while others attempt to provide more comprehensive frameworks, including activity aspects. For instance, the ASTM E1588 focuses only on the analytical approach for attributing the origin of residues as firearm discharge rather than from any other source. The current standard for GSR analysis utilizes Scanning Electron MicroscopyEnergy Dispersive X-Ray Spectrometry (SEM-EDS) to automatically scan the sample's surface and flag possible IGSR particles of interest. ${ }^{3}$ Then a forensic scientist manually reviews the discovered particles to confirm if they meet the standard criteria for classification of IGSR based on their elemental composition and morphology. The standard provides specific combinations of elements for leaded and lead-free ammunition to classify the particles according to the confidence on the compositions originating from a gunshot residue as opposed to other residues not related with the discharge of a firearm (Table 1 in section 1.2 of the introduction). For example, a "characteristic" particle is the most discriminating classification category since it contains a 
combination of elements specific to IGSR and not as common in the environment. According to the standard, a "characteristic" leaded IGSR particle contains $\mathrm{Pb}$-Sb-Ba, and a lead-free particle can contain various combinations, like Gd-Ti-Zn and Ga-Cu-Sn. The next classification is "consistent with GSR" and is slightly less discriminating since fewer or less distinctive element profiles are considered. Finally, the "commonly associated" is the least discriminating types of particles with only one element being present. Also, the addition of spheroid particle shape strengthens the confidence in the particle being IGSR, due to the nature of formation where the particles cool rapidly after discharge and their cohesive forces cause the particle to form a sphere. ${ }^{3}$ While the morphology is an important aspect of GSR detection, the standard acknowledges that IGSR can be irregular, so the more important aspect of identification is the specific combination of elements detected in the particle. Although the established criteria provide standardization in the reporting, the process still depends on human judgement, particularly when expressing the significance of the findings. This becomes particularly challenging when considering the complex mechanisms of transfer and persistence of the microscopic residues. As a result, the field can benefit from the incorporation of more comprehensive statistical approaches.

While SEM-EDS is currently the gold standard for IGSR analysis, the method does face some challenges. The turn-around-time for analysis and case reporting can take weeks to months, therefore delaying leads and investigation of these firearm-related crimes that could pose a risk to public safety. ${ }^{1}$ Also, the analysis chamber is limited to certain types of samples and requires highvacuum. ${ }^{4,5}$ This poses a risk for other forms of GSR, such as OGSR, to be lost once the sample is placed under vacuum. ${ }^{6}$ Due to these challenges, the forensic community is looking for rapid, complementary techniques to increase the workflow efficiency of GSR analysis.

One emerging technique that has gained attention in the field is Laser Induced Breakdown Spectroscopy (LIBS). ${ }^{7-16}$ LIBS is an attractive complementary technique because it is rapid $(<2$ minutes a sample), provide multielemental detection of emission lines from the infrared to ultraviolet region, allows particles analysis with spatial information on the micrometer scales, does not require sample preparation, and does minimal destruction to the sample so further analysis can be performed. An added advantage of the micro-spatial LIBS technique developed by our laboratory is the sample collection utilizes the same collection technique currently employed in the field and used for SEM-EDS analysis. ${ }^{16}$ Lastly, recent advancements in technology make the 
use of portable LIBS in the field a not so distant possibility for firearm-related crime investigation. ${ }^{17-20}$

In addition to the instrumental challenges facing the detection of IGSR, there is also the challenge of certain professionals and hobbies complicating the detection of IGSR due to the activities creating IGSR-like residues or actually coming into contact with IGSR common on the surfaces around them. Mechanics have been studied extensively because the residues created from brake pads contain spheroid particles of $\mathrm{Ba}$ and $\mathrm{Sb}$ arising from the a heat stabilizer (barium sulfate) and a solid lubricant (antimony trisulfide). ${ }^{21,22}$ Firework users have also been sampled in previous research since the conditions that fireworks undergo (high temperature and pressure, then rapid cooling) along with the types of elements used to create them ( $\mathrm{Sb}, \mathrm{Ba}, \mathrm{Pb}, \mathrm{S}, \mathrm{Ti}, \mathrm{Zn}$ ) can mimic IGSR, particularly the lead-free elemental combinations. ${ }^{22-24}$ Lastly, police officers and the stations can be a potential source of contamination since there are background levels of IGSR on the surfaces they regularly come into contact with during their shift. ${ }^{25-30}$ All these potential sources of false positives must be included in a large population study in order to build more robust classification methods and gain a more complete understanding of elemental levels present in the environment.

The scientific community is also researching the application of probabilistic approaches and to assist with more objective interpretation of scientific data. ${ }^{31-33}$ Our study uses machine learning tools to classify a sample as GSR or no GSR based on their elemental compositions and using the probability output to further inform about the weight of the evidence. This can be exploited to create probability density functions and likelihood ratios to provide the community with more objective interpretation approaches. ${ }^{34-37}$ Naïve Bayes and logistic regression are commonly used in other disciplines to assist with automated classification of data, such as emails or medical diagnosis. ${ }^{38,39}$ A more recent, but powerful, classification method are neural networks, while they are more complicated computationally, they are effective classifiers that recognize the underlaying information of the data to separate the data between possible groups of interest using methods that mimics the communication of neurons in the human brain. ${ }^{36,40,41}$ These machine learning methods combined with representative datasets can greatly assist the detection and interpretation of GSR evidence. 


\subsubsection{Current versus Bayesian Interpretation for GSR Evidence}

As discussed in the previous section, the current ASTM standards for IGSR identification is based on a three-tiered categorical classification system. ${ }^{3}$ Particles detected during automated discovery via an SEM-EDS are classified based on the elemental combinations detected and reviewed by an analyst to confirm the elements and morphology of the particle. The strongest level of classification (characteristic) contains at least three elements detected within a single particle. The next strongest classification (consistent with) is when a particle contains two elements within a single particle, exception of $\mathrm{Sr}$ in lead-free ammunition, and the weakest classification (commonly associated) is when a particle only contains one element with compositions that are also commonly found in environmental particles from numerous sources. Classification is further based on the shape of the particle, since the spheroid shape is indicative of the high temperature and pressure of formation combined with the rapid cooling as the particles travel through the air. ${ }^{42}$ Still, the current standard cautions analysts that spheroid shape alone does not indicate GSR, and the elemental combinations are necessary and the more discriminating feature for classifying a particle as GSR. Moreover, due to random process of particle formation, authentic GSR samples also contain a large portion of irregular particles.

The current standard is based on decades of IGSR research and sound scientific practice, but it is only a guide for detecting IGSR and does not provide a full guide for interpreting the collected sample as evidence. There are additional factors to consider when interpreting trace evidence beyond only identifying a submitted sample for the presence or absence of GSR. An analyst should consider the formation, collection, and transfer of the trace, such as possible source of true GSR contamination, GSR-like particles present in the environment, and the transfer and persistence nature of GSR. ${ }^{33}$ For this reason, researchers have begun to look at more robust statistics and methods to strengthen interpretation of GSR through the use of probablities. ${ }^{35-37,43-}$ ${ }^{45}$ When applying the probability to interpretation, there is a degree of belief attached that a certain fact occurred. ${ }^{45}$

Bayesian interpretation is a useful approach to better communicate the statistical weight of evidence and conveying the weight appropriately to the stakeholders and triers of fact. An analyst has the ability to express the probability of evidence given specific hypotheses, and then weigh the two hypothesis in the form of a likelihood ratio with a numerical value. ${ }^{35,46-48}$ This is important in forensic science because the court system and triers of fact want to understand the importance and 
weight of a piece of evidence, which can greatly influence the charges or verdict. ${ }^{49,50}$ Bayesian interpretation also allows the consideration of the nature of GSR creation and its transfer and persistence. ${ }^{48}$ We can assess the evidence at multiple levels when considering these factors, also referred to as the hierarchy of propositions, the mutually exclusive and exhaustive hypothesis from the prosecutor $\left(\mathrm{H}_{p}\right)$ and the defense $\left(\mathrm{H}_{d}\right)$ (Table 1). ${ }^{51-53}$ As we progress from level I to II to III, greater expertise and background information is required. ${ }^{54}$ Even though this adds some level of complexity to data analysis, there is also an increased benefit in providing a more encompassing assessment value of the evidence. Most importantly, the Bayesian frameworks are more aligned with questions of interest to the trier of fact or leading information for an ongoing investigation.

Our study in this chapter focuses on the source level I, where we are studying the two different hypothesis of attributing the source as GSR (from a leaded, lead-free, or mixed ammunition, $\mathrm{H}_{p}$ ) or the source is not GSR (from a low or high-risk background population, $\mathrm{H}_{d}$ ). Further research is underway to being to address the activity level II. However, this is out of the scope of this work, since the research in this chapter did not examine any transfer or persistence of the GSR after it was deposited, but instead focus on the elemental composition and morphology to determine if the source was or was not GSR. The findings and models proposed here are anticipated to serve as a basis of activity inferences. Also, it should be stated that level III is for the trier of fact and not on the forensic analyst to state.

Bayesian interpretation can help a scientist assess and communicate the significance of an item of evidence. This is possible by utilizing the likelihood ratio, which correlates to the probability of $\mathrm{H}_{p}$ divided by the probability of $\mathrm{H}_{d .}{ }^{35,37,41,43,44,55-57}$ In this study, we can generate the probabilities from the classification outputs of the different types of machine learning methods we chose for this study and calculate the likelihood ratio using the resulting probabilities from the algorithms. Then, further examination into the separation of the different populations can be performed by generating histograms for the different populations and the subsequent Tippet Plots for evaluating the rates of misleading evidence (i.e. false positive and false negative rates). Also, density functions displaying the spread of the likelihood ratios for the different populations can assist in learning more about the underlying data. ${ }^{46,47,58}$ The further examination of the evidence provides us with the ability to generate performance and error rates for the developed method and further strengthen the presentation of this evidence to the criminal justice system. 
Table 1: Example of hierarchy of proposition for GSR evidence. Adapted from Maitre et al. ${ }^{33}$

\begin{tabular}{|c|c|c|}
\hline Level & Type & Example of proposition \\
\hline III & Offense & $\begin{array}{l}\mathbf{H}_{p}: \text { The Person of Interest (POI) murdered the victim } \\
\mathbf{H}_{d}: \text { An unknown person murdered the victim }\end{array}$ \\
\hline II & Activity & $\begin{array}{l}\mathbf{H}_{p} \text { : The POI discharged a firearm or the reference combination of } \\
\text { firearm-ammunition } \\
\mathbf{H}_{d} \text { : An unknown person discharged a firearm or the reference } \\
\text { combination of firearm-ammunition }\end{array}$ \\
\hline I & Source & $\begin{array}{l}\mathbf{H}_{p} \text { : The trace sample came from a discharged firearm and is GSR } \\
\mathbf{H}_{d}: \text { The trace sample came from a non-related environmental } \\
\text { source and is not GSR }\end{array}$ \\
\hline
\end{tabular}

\subsubsection{Goals of the Population Study}

This study aims to assist in understanding the common elemental background of the general population, as compared to high-risk background population, and samples from known shooters originating from both leaded and lead-free ammunition. To the best of our knowledge, this study represents one of the largest population studies on gunshot residues, with nearly 3,000 samples and over 80,000 spectral datafiles used to train and test the classification algorithms. All the samples were analyzed first by LIBS as a screening method, and then confirmed by SEM-EDS using a subset of the stubs. The LIBS results were then analyzed using different classification techniques and performance rates were calculated. The probability outputs of the machine learning algorithms open the opportunity for comprehensive probabilistic interpretation of the data as a proxy for the weight of the evidence.

\subsection{Materials and Methods}

\subsubsection{Sample Collection for the Population Study}

During completion of this population study, over 2,900 samples from 975 individuals were collected and analyzed by LIBS and a smaller subset were also confirmed by SEM-EDS (208 samples, 54 low-risk, 52 lead-free, and 52 leaded completed, with 50 high-risk samples in 
progress). Each shooter set consists of four samples (left, right, palm, and back hand areas) while each background set consists of two samples (left and right hand). The collection included a population of low-risk background, leaded ammunition shooter, and lead-free ammunition samples. In addition, a data set of mixed ammunition (standard and lead-free) was collected and it is used to expand the classification capabilities of the method. Collection was performed by the individuals included on the human subject sampling protocol approved for our research by the Institutional Review Board (WVU IRB protocol \# 1506706336). Collection of the high-risk background group (mechanics, agriculture, and people who handle firearms as a part of their profession) was delayed due to COVID restrictions, so preliminary analysis by LIBS has been completed and confirmation by SEM-EDS is in progress. Figures 1 and 2 provide a breakdown of the data sets collected in this study.

All data sets were collected using the standard GSR practice for evidence collection. Residues were collected using a typical GSR stub with carbon adhesive tape on an aluminum SEM stub/mount and stored in a plastic holder. Each set of shooter samples consisted of four stubs: left palm, left back, right palm, and right back. Each set of non-shooter/background set consists of two stubs: left hand and right hand. Also, blank negative control samples were collected at the beginning and end of each collection day from the individuals who were conducting the sampling protocol.

The known-shooter collection included a variety of samples collected in different locations. The collection at the WVU ballistics laboratory (OGH) shooting range was a more controlled environment with the ability to load specialty ammunition and perform the collection indoors. Shooters would fire five rounds of ammunition inside the ballistics lab under the supervision of the range officer. The volunteers would exit the laboratory to have hand samples collected by IRBapproved collectors who were wearing Tyvek suits and gloves to reduce cross-contamination.

The specifics of the location, population, and when applicable, the type of ammunition collected can be found in Table 2. The type of ammunition fired was a combination of lead-free, leaded, specialty loaded, and manufactured ammunition, and either a pistol or revolver were used as the firearm. The specialty loaded ammunition was made with some of the primers characterized and described in chapter 2 . When the ammunition type was changed during collection, the firearm was cleaned using a solvent to remove build-up from the barrel and decease contamination from 
the previous type of ammunition. Also, a completely different barrel was used when shooting leadfree ammunition to reduce the memory effects from previous shooting events.

The second major collection site was at the World Scout Jamboree (WSJ), which was conducted in an outdoor setting with different type of ammunition defined by the organizers of the event, but out of our control. Also, in this shooting site, the number of shots being fired, the last time participants had washed their hands, knowledge of post-shooting activities, or the demographic of the subjects being sampled was not known or documented. These conditions therefore represented a set of realistic situations that may be involved in a case. The outdoor range consisted of multiple pistol ranges where the participants could fire anywhere from 10 to 40 shots using either a .22 caliber or $9 \mathrm{~mm}$ pistol or both. The large volume of people at the event allowed us to collect samples from many individuals as the they were leaving the pistol ranges. Each collected sample originated from a different individual. This sample set also had the additional challenge of being a mixture of leaded and lead-free ammunition. The participants could fire both types of firearm and ammunition or just one, so the collection process could not be controlled for this variable. Nonetheless, the sample set represents valuable information about mixed ammunition and was used for training and testing the machine learning algorithms.

The backgrounds were collected using the same protocol. All the participants were asked if they had fired a gun within the last 24 hours and no handwashing took place before collection (exception of 10 additional sets collected in Oglebay Hall for sample collection comparison purposes). The samples without handwashing provide a better representation of the background elements on the hands of the low-risk population. The samples collected on the WVU downtown campus $(\mathrm{OGH})$ were collected both indoors and outdoors, while the WSJ samples were collected outdoors in a WVU tent located in the visitor's area several miles away from the shooting ranges. The participants were not asked when they had last washed their hands, but they did have access to handwashing facilities at both locations.

The high-risk background samples were collected from multiple locations around Morgantown, WV. Samples were only collected from those who confirmed they had not fired a weapon within the last 24 hours. The professions and hobbies chosen were based on existing literature and our ability to access diverse groups of interest. Three different groups were sampled: people working with soils and fertilizers, officers and researchers who handle firearms or come in contact with possible GSR transfer due to their jobs, and mechanics who come in contact with 
brake pad residues and tires. A total of 21, 65, and 19 sets were collected for each group respectively to establish the elemental profiles on their hand due to the type of residues they come into contact throughout the day. 


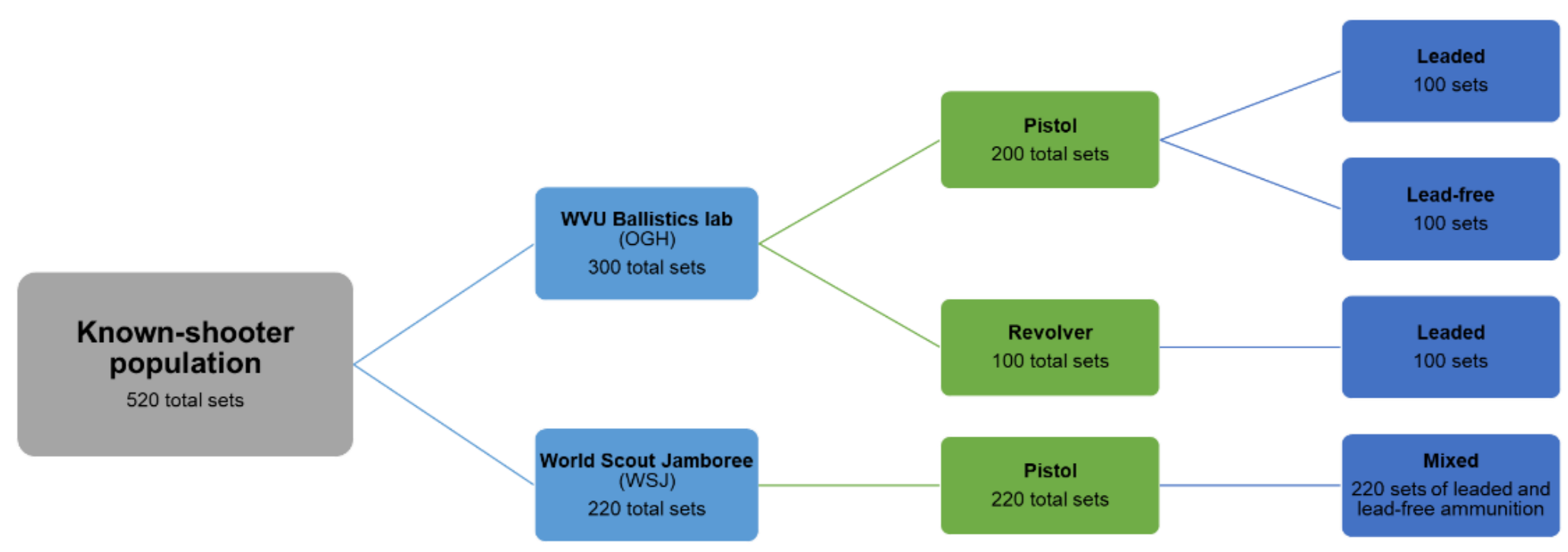

Figure 1: Flowchart of the samples collected for the known-shooter population of the study. Each set consists of four samples: right back, right palm, left back, and left palm. 


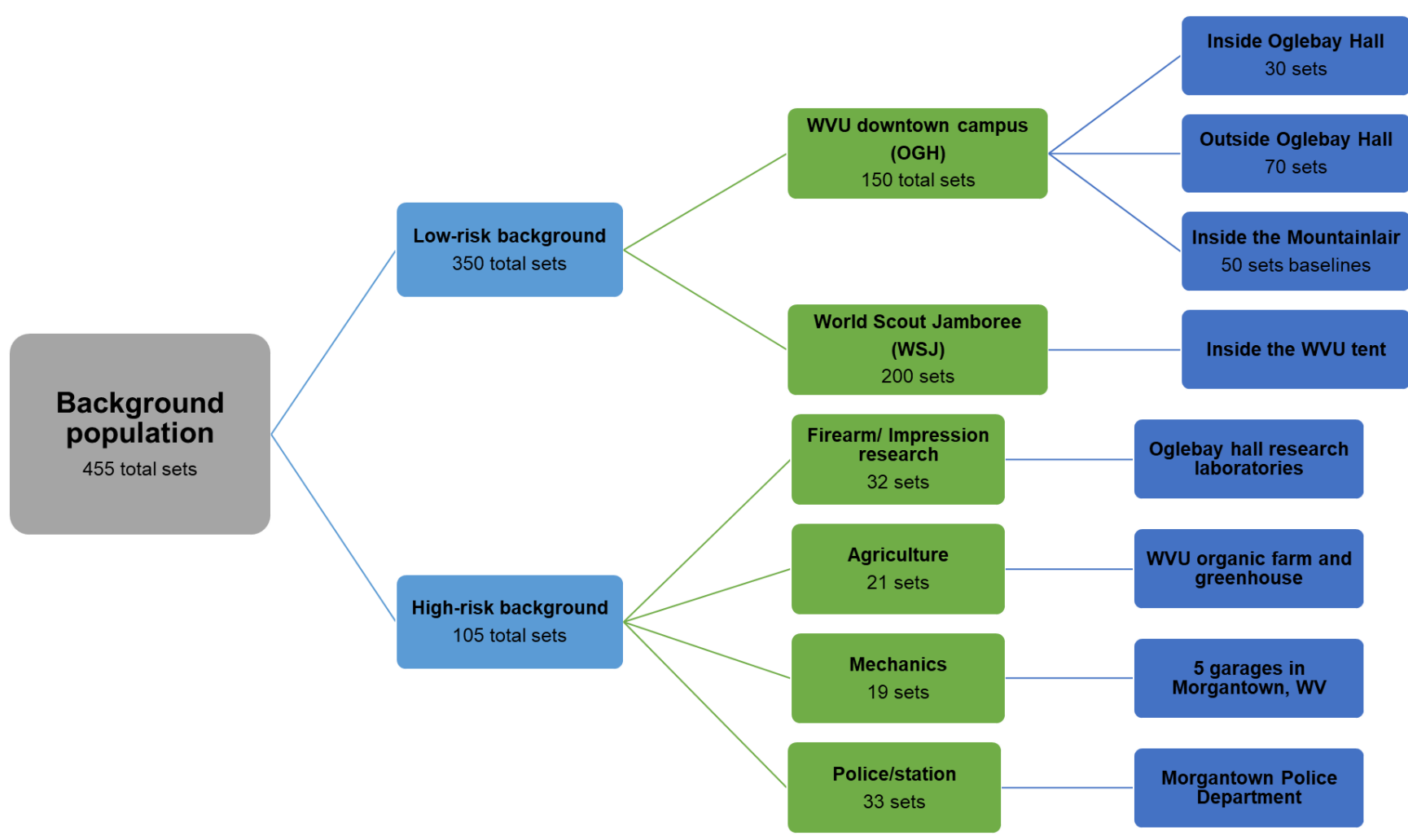

Figure 2: Flowchart of the samples collected for the background population of the study. Each set consists of two stubs: right hand and left hand, except for the 50 samples collected in the Mountainlair from only the dominant hand. 
Table 2: Summary of the different types of firearm and ammunition used for the standard ammunition population portion of the study.

\begin{tabular}{|c|c|c|c|c|c|c|c|c|}
\hline Firearm & $\begin{array}{l}\text { Type of } \\
\text { Firearm }\end{array}$ & Type of Ammunition & $\begin{array}{l}\text { Lead- } \\
\text { free }\end{array}$ & Caliber & $\begin{array}{c}\text { Sets } \\
\text { Collected }\end{array}$ & $\begin{array}{c}\text { Set } \\
\text { Number }\end{array}$ & $\begin{array}{c}\text { Location } \\
\text { (abbreviation) }\end{array}$ & $\begin{array}{c}\text { Date of } \\
\text { Collection }\end{array}$ \\
\hline N/A & N/A & $\begin{array}{c}\text { Background samples } \\
\text { (low-risk) }\end{array}$ & N/A & N/A & 20 & 001:020 & $\begin{array}{l}\text { Oglebay Hall } \\
\text { (OGH) }\end{array}$ & 052419 \\
\hline $\begin{array}{l}\text { Springfield } \\
\text { XD9 }\end{array}$ & Pistol & $\begin{array}{l}\text { Reloaded and } \\
\text { unknown }\end{array}$ & No & $9 \mathrm{~mm}$ & 20 & 001:020 & $\begin{array}{l}\text { WVU Ballistic } \\
\text { Lab } \\
(\mathrm{OGH})\end{array}$ & 052419 \\
\hline $\begin{array}{l}\text { Springfield } \\
\text { XD9 }\end{array}$ & Pistol & $\begin{array}{c}\text { Manufacturer loaded } \\
\text { Blazer }\end{array}$ & No & $9 \mathrm{~mm}$ & 20 & 021:040 & $\begin{array}{l}\text { WVU Ballistic } \\
\text { Lab } \\
(\mathrm{OGH})\end{array}$ & 060319 \\
\hline N/A & N/A & $\begin{array}{c}\text { Background samples } \\
\text { (low-risk) }\end{array}$ & N/A & N/A & 10 & 021:030 & $\begin{array}{l}\text { Oglebay Hall } \\
(\mathrm{OGH})\end{array}$ & 060319 \\
\hline $\begin{array}{l}\text { Springfield } \\
\text { XD9 }\end{array}$ & Pistol & $\begin{array}{l}\text { Specialty loaded } \\
\text { Remington }\end{array}$ & No & $9 \mathrm{~mm}$ & 40 & 041:080 & $\begin{array}{l}\text { WVU Ballistic } \\
\text { Lab } \\
(\mathrm{OGH})\end{array}$ & 061119 \\
\hline N/A & N/A & $\begin{array}{c}\text { Background samples } \\
\text { (low-risk) }\end{array}$ & N/A & N/A & 38 & 031:068 & $\begin{array}{c}\text { Outside Oglebay } \\
\text { Hall (OGH) }\end{array}$ & 062019 \\
\hline N/A & N/A & $\begin{array}{l}\text { Background samples } \\
\text { (low-risk) }\end{array}$ & N/A & N/A & 32 & 069:100 & $\begin{array}{c}\text { Outside Oglebay } \\
\text { Hall (OGH) }\end{array}$ & 062419 \\
\hline $\begin{array}{l}\text { SIG SAUER } \\
\text { P320 and } \\
\text { Ruger Mark } \\
\text { IV }\end{array}$ & Pistols & $\begin{array}{c}\text { Manufacturer loaded } \\
\text { Federal and } \\
\text { SYNTECH }\end{array}$ & $\underset{\text { d }}{\text { Mixe }}$ & $\begin{array}{c}.22 \mathrm{LR} \\
\text { and } 9 \\
\mathrm{~mm}\end{array}$ & 220 & $081: 328$ & $\begin{array}{c}\text { World Scout } \\
\text { Jamboree (WSJ) }\end{array}$ & $\begin{array}{l}072919 \text { and } \\
073019\end{array}$ \\
\hline N/A & N/A & $\begin{array}{l}\text { Background samples } \\
\text { (low-risk) }\end{array}$ & N/A & N/A & 200 & $101: 300$ & $\begin{array}{c}\text { World Scout } \\
\text { Jamboree (WSJ) }\end{array}$ & $\begin{array}{c}072919 \text { and } \\
073019\end{array}$ \\
\hline $\begin{array}{l}\text { Springfield } \\
\text { XD9 }\end{array}$ & Pistol & $\begin{array}{l}\text { Specialty loaded } \\
\text { Winchester }\end{array}$ & No & $9 \mathrm{~mm}$ & 20 & $329: 348$ & $\begin{array}{c}\text { WVU Ballistic } \\
\text { Lab (OGH) }\end{array}$ & 091119 \\
\hline $\begin{array}{c}\text { Taurus } \\
\text { Model } 608\end{array}$ & Revolver & $\begin{array}{l}\text { Specialty loaded } \\
\text { Remington }\end{array}$ & No & $9 \mathrm{~mm}$ & 50 & $349: 398$ & $\begin{array}{c}\text { WVU Ballistic } \\
\text { Lab (OGH) }\end{array}$ & 112119 \\
\hline $\begin{array}{c}\text { Taurus } \\
357 \text { Magnum }\end{array}$ & Revolver & $\begin{array}{c}\text { Manufacturer loaded } \\
\text { American Eagle } \\
\text { Federal }\end{array}$ & No & $\begin{array}{c}38 \\
\text { Special }\end{array}$ & 50 & 399:448 & $\begin{array}{c}\text { WVU Ballistic } \\
\text { Lab (OGH) }\end{array}$ & 121019 \\
\hline N/A & N/A & $\begin{array}{l}\text { Background samples } \\
\text { (low-risk) }\end{array}$ & N/A & N/A & 50 & $301: 350$ & $\begin{array}{c}\text { Outside Oglebay } \\
\text { Hall (OGH) }\end{array}$ & 121219 \\
\hline $\begin{array}{l}\text { Springfield } \\
\text { XD9 }\end{array}$ & Pistol & $\begin{array}{l}\text { Specialty loaded } \\
\text { Fiocchi lead-free }\end{array}$ & Yes & $9 \mathrm{~mm}$ & 20 & 041:060 & $\begin{array}{c}\text { WVU Ballistic } \\
\text { Lab (OGH) }\end{array}$ & 062220 \\
\hline $\begin{array}{l}\text { Springfield } \\
\text { XD9 }\end{array}$ & Pistol & $\begin{array}{l}\text { Specialty loaded CCI } \\
\text { lead-free }\end{array}$ & Yes & $9 \mathrm{~mm}$ & 20 & 061:080 & $\begin{array}{c}\text { WVU Ballistic } \\
\text { Lab (OGH) }\end{array}$ & 062220 \\
\hline $\begin{array}{l}\text { Springfield } \\
\text { XD9 }\end{array}$ & Pistol & $\begin{array}{l}\text { Specialty loaded CCI } \\
\text { lead-free }\end{array}$ & Yes & $9 \mathrm{~mm}$ & 14 & 090:103 & $\begin{array}{c}\text { WVU Ballistic } \\
\text { Lab (OGH) }\end{array}$ & 062920 \\
\hline
\end{tabular}


Table 2 continued

\begin{tabular}{|c|c|c|c|c|c|c|c|c|}
\hline Firearm & $\begin{array}{c}\text { Type of } \\
\text { Firearm }\end{array}$ & Type of Ammunition & $\begin{array}{l}\text { Lead- } \\
\text { free }\end{array}$ & Caliber & $\begin{array}{c}\text { Sets } \\
\text { Collected }\end{array}$ & $\begin{array}{c}\text { Set } \\
\text { Number }\end{array}$ & $\begin{array}{c}\text { Location } \\
\text { (abbreviation) }\end{array}$ & $\begin{array}{c}\text { Date of } \\
\text { Collection }\end{array}$ \\
\hline $\begin{array}{l}\text { Springfield } \\
\text { XD9 }\end{array}$ & Pistol & $\begin{array}{c}\text { Manufacturer loaded } \\
\text { Hevi-Shot }\end{array}$ & Yes & $9 \mathrm{~mm}$ & 2 & 031:032 & $\begin{array}{l}\text { WVU Ballistic } \\
\text { Lab (OGH) }\end{array}$ & 062920 \\
\hline $\begin{array}{l}\text { Springfield } \\
\text { XD9 }\end{array}$ & Pistol & $\begin{array}{l}\text { Manufacturer loaded } \\
\text { SYNTECH }\end{array}$ & Yes & $9 \mathrm{~mm}$ & 30 & $\begin{array}{c}\text { 033:040: } \\
\text { 081:089; } \\
\text { 104:116 }\end{array}$ & $\begin{array}{l}\text { WVU Ballistic } \\
\text { Lab (OGH) }\end{array}$ & 071620 \\
\hline $\begin{array}{l}\text { Springfield } \\
\text { XD9 }\end{array}$ & Pistol & $\begin{array}{l}\text { Specialty loaded } \\
\text { Fiocchi lead-free }\end{array}$ & Yes & $9 \mathrm{~mm}$ & 14 & 117:130 & $\begin{array}{l}\text { WVU Ballistic } \\
\text { Lab (OGH) }\end{array}$ & 071620 \\
\hline N/A & N/A & $\begin{array}{c}\text { Background samples } \\
\text { (high-risk } \\
\text { firearm/impression } \\
\text { research) }\end{array}$ & N/A & N/A & 32 & 001:032 & $\begin{array}{l}\text { Oglebay Hall } \\
\text { research } \\
\text { laboratory } \\
(\mathrm{OGH})\end{array}$ & $\begin{array}{c}030921 \text { and } \\
031021\end{array}$ \\
\hline N/A & N/A & $\begin{array}{l}\text { Background samples } \\
\text { (high-risk agriculture) }\end{array}$ & N/A & N/A & 21 & $\begin{array}{c}033: 046 \\
\text { and } \\
066: 072\end{array}$ & $\begin{array}{l}\text { WVU organic } \\
\text { farm and } \\
\text { greenhouse }\end{array}$ & $\begin{array}{c}031821 \text { and } \\
033021\end{array}$ \\
\hline N/A & N/A & $\begin{array}{l}\text { Background samples } \\
\text { (high-risk mechanics) }\end{array}$ & N/A & N/A & 19 & 047:065 & $\begin{array}{l}5 \text { mechanic } \\
\text { garages around } \\
\text { Morgantown, } \\
\text { WV }\end{array}$ & 033021 \\
\hline N/A & N/A & $\begin{array}{c}\text { Background samples } \\
\text { (high-risk } \\
\text { police/station) }\end{array}$ & N/A & N/A & 33 & 073:105 & $\begin{array}{l}\text { Morgantown } \\
\text { Police } \\
\text { Department }\end{array}$ & 033121 \\
\hline
\end{tabular}

\subsubsection{Quality Control Samples for Instrument Daily Performance}

Every automated GSR run by SEM-EDS included four stubs of interest (either shooter or background), one blank, and one synthetic GSR QC sample. The blank served as a negative control to demonstrate there was no GSR or GSR-like residues present in the laboratory or SEM chamber. The GSR QC sample was a positive control included between each analyzed stub to ensure the performance of the instrument remained constant during the long, automated analysis time. This was demonstrated by analyzing the same area on the QC stub and receiving the same number and classification of particles in the area.

Before performing LIBS analysis, a series of tailor-made $p$ GSR standards were analyzed to demonstrate the instrument's performance for the detection of IGSR. Three tailor-made $p$ GSR standards were analyzed, one with $\mathrm{Pb}$ but no $\mathrm{Ba}$, one with lower $\mathrm{Pb}$ and more $\mathrm{Ba}$, and one with $\mathrm{Ti}$ and $\mathrm{Zn}$ for lead-free ammunition types. These intensities were compared with control charts, and the number of positive spots based on the elements present was monitored during data analysis. Also, a blank of only carbon adhesive was run to ensure there was no interference coming from 
the adhesive surface or environmental contamination in the laboratory and LIBS ablation cell. Negative control samples gathered during the hands' sampling were also monitored to demonstrate clean conditions during the actual collection steps.

\subsubsection{Instrumental Analysis}

Inorganic GSR (IGSR) particles were identified and analyzed by both the micro-spatial LIBS method presented in chapter $3^{16}$ and SEM-EDS using the American Society for Testing and Materials standards as a guideline for classification of particles. ${ }^{3}$ SEM-EDS analysis was conducted on a JEOL 6490LV (Peabody, MA) in accordance with ASTM 1588-20 standard for the analysis of GSR. The manufacturer SEM user interface software was version 8.14. The instrumental parameters used during analysis and spectra collection consisted of an accelerating voltage of $25 \mathrm{kV}$, a spot size of $60 \mu \mathrm{m}$, a working distance of approximately $18 \mathrm{~mm}$, and a magnification of 500x. A backscatter and a secondary electron detector were used to image particles, while an Oxford Instrument INCAx-sight 7623 Energy Dispersive X-ray Spectroscopy (EDS, England) detector collected elemental information about the particles of interest. After consulting with our crime laboratory collaborators, a cutoff of 10 characteristic particles was included in the run to simulate protocols used by some laboratories and decrease the amount of analysis time per stub.

A J200 Tandem LIBS system (Applied Spectra, CA) equipped with a $266 \mathrm{~nm}$, high-power, Q-switched, Neodymium doped Yttrium Aluminum Garnet (Nd YAG) nanosecond laser was used for analysis. The instrument detector optics and sensor consisted of a six-channel Czerny-Turner spectrometer with a spectral range from 190 to $1040 \mathrm{~nm}$ and a Charge Coupled Device (CCD) based broadband detector, respectively. A second J200 tandem LIBS instrument almost identical to the one described was also used on a sub-set of the samples at one of our industry collaborator's site. The only difference was a second Intensified CCD (ICCD) based detector in the UV range of the spectrum was used for increased resolution and sensitivity.

The prepared samples were placed in the ablation chamber using a custom stage platform with a hole in the center that securely held the pin mount; this ensured that the surface remained even during analysis to maintain a consistent focus on the stub surface. The chamber was purged for 45 seconds with $1 \mathrm{~L} / \mathrm{min}$ of argon (ultrahigh purity, Matheson, WV) before performing analysis. 


\subsubsection{Data Analysis}

The SEM-EDS experiment was designed to monitor the elemental composition and morphology of the GSR particles. The analysis was automated and organized by INCAx-sight software (Microanalysis Suite Issue 17b+SP2 and Version 4.09: Concord, MA), which is designed for the management of image and spectral data. Images of represented features, and their respective spectra, were collected for each stage of the study. Particle count and size was also documented as metadata in the study.

The data analysis software Aurora (Applied Spectra, CA) was used to crop the collected spectra in order to isolate the desired spectral regions. Background subtraction, peak identification, and peak integration on the peaks of interest were subsequentially performed. The integrated peak areas were imported into Excel ProPlus (Microsoft 365 MSO 16.9.13127.21336 64-bit) and combined into one workbook. Signal-to-noise ratios (SNR) for all the emission lines of interest (Iatomic and II- ionic) - Al $394.4 \mathrm{~nm}$ (I), Al $396.1 \mathrm{~nm}$ (I), Ba $455.4 \mathrm{~nm}$ (II), Ba $493.4 \mathrm{~nm}$ (II), Ba 614.2 nm (II), Ba 649.7 nm (II), Bi 293.8 nm (I), Bi 412.1 nm (I), Bi 472.2 nm (I), Cu 324.7 nm (I), Cu 327.4 nm (I), Cu 515.3 nm (I), K 766.4 nm (I), K 769.9 nm (I), Pb 368.3 nm (I), Pb 405.8 nm (I), Sb 252.8 nm (I), Sb 259.8 nm (I), Sb 287.8 nm (I), Sb 326.7 nm (I), Si 251.4 nm (I), Si $288.1 \mathrm{~nm}$ (I), Si $390.5 \mathrm{~nm}$ (I), Sn $284.0 \mathrm{~nm}$ (I), Sn $317.5 \mathrm{~nm}$ (I), Sn $326.2 \mathrm{~nm}$ (I), Ti 334.9 nm (II), Ti 368.5 nm (II), Ti 375.9 nm (II), Ti 376.1 nm (II), Zn 328.2 nm (I), Zn 334.5 nm (I), Zn 481.0 $\mathrm{nm}$ (I)-were calculated by using the background of the spectrum in proximity to the peak of interest. The SNR for the most informative peaks of Al $(396.1 \mathrm{~nm}), \mathrm{Ba}(455.4 \mathrm{~nm}), \mathrm{Bi} 472.2 \mathrm{~nm}$ (I), Cu (324.7 nm), K 766.4 nm (I), Pb (405.8 nm), Sb (259.8 nm), Si $288.1 \mathrm{~nm}$ (I), Sn $326.2 \mathrm{~nm}$ (I), Ti $334.9 \mathrm{~nm}$ (II), and Zn $481.0 \mathrm{~nm}$ (I) were imported into Excel for data pre-processing before statistical testing.

\subsubsection{Statistical Analysis}

The performance rates for the micro-spatial LIBS methods were evaluated using four approaches. First, an exploratory critical threshold method was used to evaluate the data and examine the variance and mean of the different groups. This was calculated in the same manner as in Trejos et. al. ${ }^{14}$ For the micro-spot method, analysis was performed on one stub from each control-shooter sample and each analysis produced 25 spectra per stub due to the $5 \times 5$ spot grid pattern as reported in Menking Hoggatt et al. ${ }^{16}$ Three classifier methods were used in this study to 
evaluate their ability to predict class membership: Logistic Regression (LR), Naïve Bayes (NB) and Neural Networks. The software JMP®Pro 15.1.0 (version 426298) provided the results of the algorithms in the form of confusion matrices, which were used to calculate the performance rates.

All three machine learning models were supervised prediction methods, and the data was separated into $60 \%$ of the data for training, $20 \%$ for validation, and $20 \%$ for testing. The selection of the data was randomly assigned and repeated 5-10 times. Logistic regression classifies the data based on a threshold determined by the training data set and logistic function. Naïve Bayes provides the probability of a data vector belonging to a specific group by using the training data to determine the class and assuming that the predictor variables are independent. The neural network used in this study consisted of 16 emission lines of interest and two layers, one linear layer for data reductions and one TanH layer (similar to LR sigmoid threshold shape) to classify the data. The performance rates were compared to determine accuracy of each of the machine learning methods studied.

The machine learning algorithms also provided an output in the form of a probability, which were used to estimate kernel density functions and likelihood ratios as a proxy to assess the weight of the evidence. Probabilities for the logistic regression and neural network machine learning were exported from the JMP Pro 15 and read into R (version 4.0.5) and user interface RStudio (version 1.2.1335). Histograms, Tippet plots, and Kernel Density Functions (KDF) were plotted using the $\log 10$ of the likelihood ratios and assisted in visualizing the performance of the methods and the amount of misleading evidence. ${ }^{46}$ Two likelihood ratios were calculated: one considered each shooter population (leaded, lead-free, and mixed ammunition) separately (Equation 1) and one considered all the shooter populations combined (Equation 2). The rationale of using two approaches to estimate the LR is in real casework, the examiner would not always be able to know the type of ammunition used to generate the GSR residue.

The data from the ground truth shooter population is considered for $\mathrm{H}_{p}$ and changes based on the likelihood ratio being calculated as explained above, where the low-risk population is considered for $\mathrm{H}_{d}$ and remains constant in the denominator. The high-risk population is in progress and currently not included in the probabilistic interpretation. 
Equation 1: Likelihood ratio formula 1 where the shooter changes based on the population being considered (leaded, lead-free, or mixed).

$$
\text { Likelihood Ratio } 1=\frac{P\left(E \mid H_{p \text { shooter }}\right)}{P\left(E \mid H_{\text {d low-risk }}\right)}
$$

Equation 2: Likelihood ratio formula 2 where all three shooter populations are considered simultaneously.

$$
\text { Likelihood Ratio } 2=\frac{\left[P\left(E \mid H_{\text {pleaded }}\right), P\left(E \mid H_{\text {plead-free }}\right), P\left(E \mid H_{\text {p mixed }}\right)\right] / 3}{P\left(E \mid H_{\text {d low-risk }}\right)}
$$

\subsection{Results and Discussion}

This study aimed to evaluate and validate the capabilities and limitations of a novel LIBS technique as a fast-screening tool for GSR examinations. The method was developed and optimized as described in our recent publication and section 5 (chapter 3 ) of this dissertation. ${ }^{16}$ In this research, we expanded the validation of the method to a larger population of nearly 3,000 authentic samples collected from individuals who have not fired a gun recently and from those who have discharged a firearm. The various ammunition and firearms were selected to represent those commonly encountered in casework. SEM-EDS was applied as a cross-validation tool, following the current ASTM standard practice to a subset of samples analyzed by LIBS beforehand. The SEM-EDS data serve as the "ground truth" for this study to confirm the results observed by LIBS.

SEM-EDS was conducted after LIBS and electrochemical (EC) analysis on the same specimens to assess the feasibility of incorporating screening testing into existing examination protocols. The electrochemical analysis has demonstrated to provide orthogonal information to the LIBS data. The combined application of LIBS/EC is part of more extensive research funded by NIJ to develop practical, fast screening analytical and chemometric approaches for firearm discharge residues detection (award \# 2018-DU-BX-0186). The electrochemistry research is part of another doctoral dissertation (Colby Ott, Dr. Luis Arroyo research group) and therefore out of the scope of this section. This chapter focuses on reporting the performance of the LIBS examination as basic foundation of the broader research objectives. However, it is important to note that the overall sequence of analysis consisted of LIBS screening followed by EC, providing information on IGSR and OGSR in under 5 minutes per sample. Due to the minimally destructive 
nature of these methodologies, the same specimen can then be analyzed by SEM-EDS if necessary. The ability to preserve the sample for confirmatory methods was essential in the early developmental and experimental design of the research.

As such, the LIBS method was envisioned as a screening tool that can lead to more effective case management, capable of aiding in fast decision-making processes at the crime scene and laboratory and informing the selection of items that may undergo SEM-EDS confirmation. Also, since the LIBS examination is completed in just a tiny fraction of the time it takes to run SEM-EDS, it can expand to other items considered time-prohibitive by current protocols, such as the face, clothing, and other surfaces, strengthening the evidence interpretation. In the following sections, we will discuss first the findings of SEM-EDS on a sub-group of samples to establish the knowledge of particle compositions on the authentic items, followed by a comprehensive description of the LIBS results and the proposed approaches for the statistical interpretation of gunshot residues.

\subsubsection{SEM-EDS Analysis on the Population}

The ASTM standard E1588-20 identifies the SEM-EDS as the gold standard for IGSR analysis. This technique is ideally suited to provide elemental and morphological information of the samples, using the SEM for imaging the particles and EDS for detecting their elemental composition. The ASTM standard establishes that characteristic or consistent GSR particles are often spheroid and ranging from $0.5 \mu \mathrm{m}$ to $5 \mu \mathrm{m}$ in diameter. The elemental composition of those particles provides additional confidence for GSR identification. When beginning to search for characteristic IGSR particles, an analyst may examine particles that appear brighter, since characteristic elements like lead, barium, and antimony, have a higher atomic weight and produce more backscatter electrons. Also, the standard practice classifies the elemental information for IGSR particles into three categories depending on the confidence to assign the sources as GSR (characteristic, consistent with, or commonly associated with GSR). In this study, we followed the traditional categorical classification of GSR particles when analyzing SEM-EDS data to gain knowledge of observed profiles on the datasets, to use the SEM-EDS information as "ground truth" for LIBS comparison, and to evaluate if the ability of SEM-EDS detection was compromised by preliminary screening testing on the same sample. 
As a result, for leaded ammunition samples, we considered particles to be "characteristic" of IGSR if they contained lead, barium, and antimony. This specific combination is rarely found in particles from any source other than GSR. Characteristic particles demonstrate the presence of IGSR, but do not indicate that the sampled individual had fired the weapon, only that they could have been in the vicinity of a shooting event. Particles that are classified as "consistent with GSR" contain only two of the characteristic elements, with silicon or calcium also sometimes being detected. Also, those that are classified as "consistent with GSR" particles may be attributed to sources other than firearm ammunition, so morphology must also be considered during analysis. Commonly associated particles contain only one of the characteristic elements, in addition to the presence of other more common elements such as potassium, zinc, or aluminum. This classification is the weakest ranking for GSR identification since these particles can derive from numerous environmental sources and its sole presence in a sample does not provide support of GSR origin.

Lead-free ammunition is challenging because it does not contain the same elements of interest as leaded ammunition and the ASTM standard does not contain a comprehensive list of elements for identification of these IGSR. As a result, categorization was based on a tailor-made microparticle standard (section 5: chapter 2), which composition was previously characterized using three different instruments (ICP-MS, LIBS, and SEM-EDS). The research of modern, leadfree ammunitions using tailor-made IGSR suspension that were characterized by our research group allowed for the creation of custom classifications for lead-free ammunition and elements not included in the ASTM method. Lead-free ammunition brands that were utilized in the discharge of firearms for the shooter's hands sets were Fiocchi, SYNTECH, and CCI.

Elements of interest targeted for classification in the Fiocchi ammunition were potassium, copper, and sometimes bismuth. When characterizing this ammunition though, bismuth was not consistently detected by Inductively Coupled Plasma-Mass Spectrometry (ICP-MS) and Laser Induced Breakdown Spectroscopy (LIBS) since it was too low in concentration (<1 ppm). Bismuth was the only consistently characteristic element in the SYNTECH ammunition, while CCI was classified based only on particles containing barium. While the ASTM standard does not even list bismuth as a possible GSR element, it does define an elemental combination of titanium and zinc as "consistent with" classification for lead-free ammunition. The Ti-Zn combination can be indicative of the elemental composition of the cartridge case, and this was also included in the 
automated IGSR classification recipe for lead-free ammunition. In the study, only a few particles contained this combination, and the elements of interest were more consistent with the elements identified by ICP-MS and previously described for each type of ammunition.

\subsubsection{Low-risk Background Dataset}

In congruence with LIBS analysis, the incidence of false positive particles by SEM-EDS on background samples was very low. Figure 3 displays the total number of particles identified by SEM-EDS for the low-risk population, originating from 56 stubs previously analyzed by LIBS. Profiles of single, binary and ternary compositions of leaded and lead-free residues were monitored for the background samples. While five particles were originally classified as characteristic and numerous other particles were given "consistent with" rankings, further data analysis excluded the particles as their morphology were not indicative of GSR. Moreover, the few particles initially considered characteristic had a combination of $\mathrm{Sb}-\mathrm{Sn}-\mathrm{Ba}$ or $\mathrm{Ti}-\mathrm{Zn}-\mathrm{Cu}$ that was established in our protocol but are not considered characteristic for leaded ammunition according to the newest ASTM standard. The background samples contain several particles of Ti-Zn, Sb-Ba, Sb-Pb, and $\mathrm{Ba}-\mathrm{Pb}$; all of these compositions classify as the "consistent" category. Interesting to note is the relatively high incidence of $\mathrm{Sr}$ and $\mathrm{Ce}-\mathrm{Fe}$ particles in the background samples, which was not observed in the shooter sets. The additional elemental combinations of the commonly associated compositions were observed in relatively similar frequency in the low-risk background samples and the leaded shooter samples (Figure 3 and section 1.4.3), yet the morphology of these particles was recorded and none of the particles were spheroid in the low-risk background, so the particles were not confirmed as GSR. The exception can be found in Figure 4. While the particle contains the typical characteristic elements, this was the only particle characteristic of GSR found on the entire stub, which could weaken the overall evidential value of an identified GSR.

Another interesting observation during the confirmation of hands samples by SEM-EDS was discovering of non-GSR micrometer-sized particles that were spheroid (Figure 5). While they lack the typical leaded characteristic elements, a particle such as this could be mistaken for a GSR particle originating from lead-free ammunition (Figure 6). Ongoing research into the changing composition of modern ammunition is essential, but equally important is the background population's characterization. These spherical microparticles from non-GSR sources are a perfect example of how important this research is to the forensic community. 


\subsubsection{Shooter hands' Residues from Leaded Ammunition Dataset}

The discovery of GSR particles on the leaded shooter population was straightforward, allowing GSR confirmation on all samples, even after analyzing the same stub by LIBS and EC. All leaded shooter samples had at least 6 characteristic particles, with a dominant typical particle composition of Sb-Ba-Pb and spheroid morphology. In the "consistent with" category, most particles were combinations of $\mathrm{Sb}-\mathrm{Pb}, \mathrm{Sb}-\mathrm{Ba}$, or $\mathrm{Ba}-\mathrm{Pb}$. Finally, in the commonly associated class, the most prominent were particles containing only $\mathrm{Ba}$ or Fe. A summary of all the elemental combinations found during confirmation can be found in Figure 7. Also, out of the 52 stubs analyzed, 47 reached the threshold of 10 potential characteristic particles not requiring monitoring the entire stub's surface. Only five stubs required scanning the whole stub because the software did not flag 10 GSR characteristic particles, but in the end of the mapping 6 characteristic particles were identified and confirmed by the examiner.

\subsubsection{Shooter hands' residues from lead-free ammunition dataset}

A challenge during the confirmation of hand samples from lead-free ammunition was the amount of "only barium" particles observed in the different populations. Barium is considered a commonly associated element in the ASTM guidelines. Figure 3 and Figure 7 show a high particle count for barium present in the leaded shooter's samples and the backgrounds. What makes this tricky is that $\mathrm{Ba}$ is one of the few elements that permit elemental identification of a GSR for some lead-free ammunition. For instance, the CCI ammunition was determined to produce GSR particles with only barium as the major IGSR element of interest in the primer (Figure 8). This is problematic for GSR identification because the ASTM standard classifies the presence of Ba as a commonly associated feature, so identifying an IGSR particle from a CCI primer could be quite challenging for interpretation and not exclusive of lead-free formulations. Moreover, in a real case, the ground truth of the ammunition type would not necessarily be known, complicating the criteria for identification of GSR residues. Upon analysis of the CCI ammunition, the morphology was analyzed to determine if particles containing barium had spherical or irregular morphology to classify as characteristic GSR for leaded-free. Only then was the sample considered positive if the particles were spheroid. Both types of morphology were observed by the analyst (Figure 9). 


\section{Low-risk Classes}

Overall

$\mathrm{n}=56$ stubs

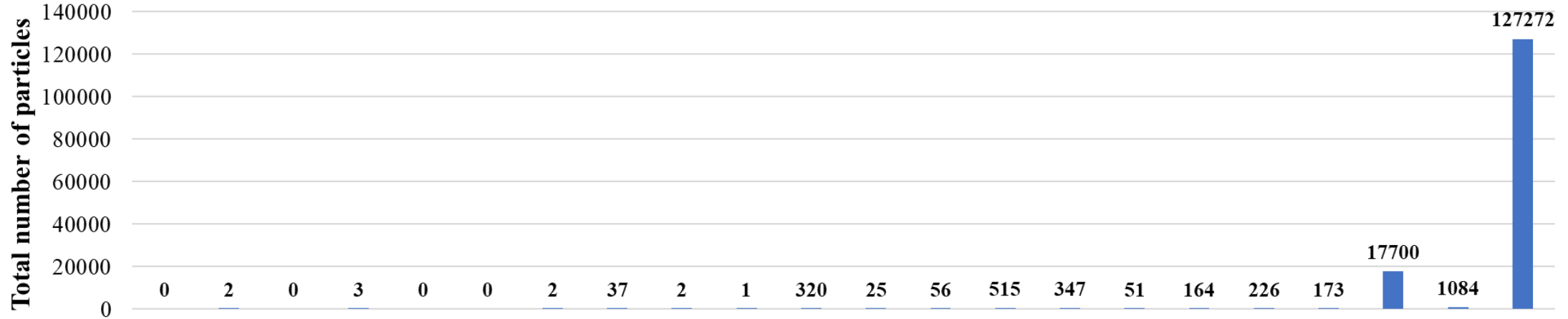

Adjusted y-axis

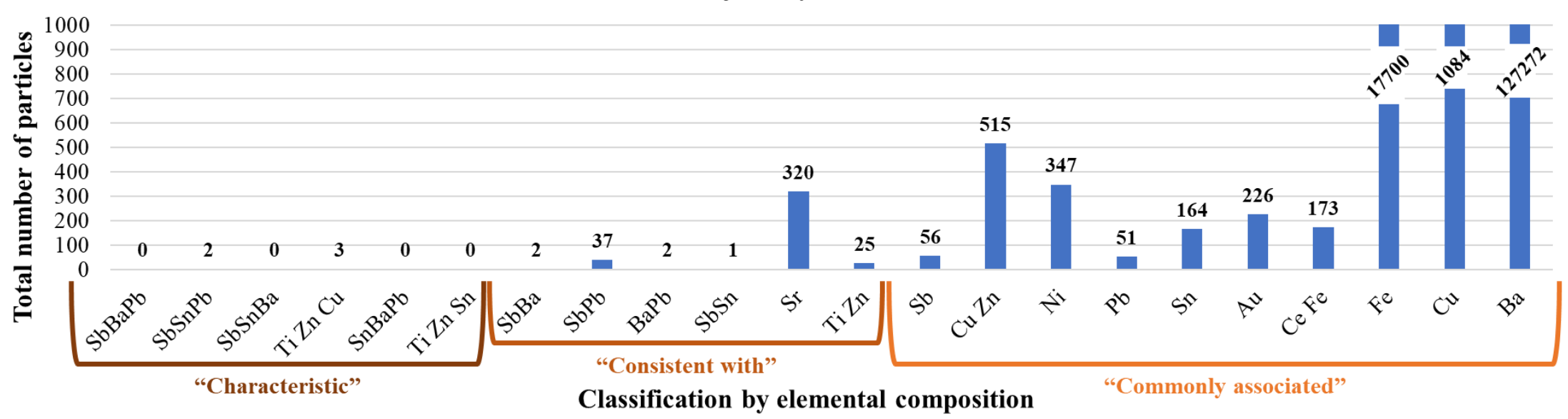

Figure 3: Low-risk background population and the classification with rankings for the total number of particles found on all the stubs analyzed $(\mathrm{n}=56)$. 


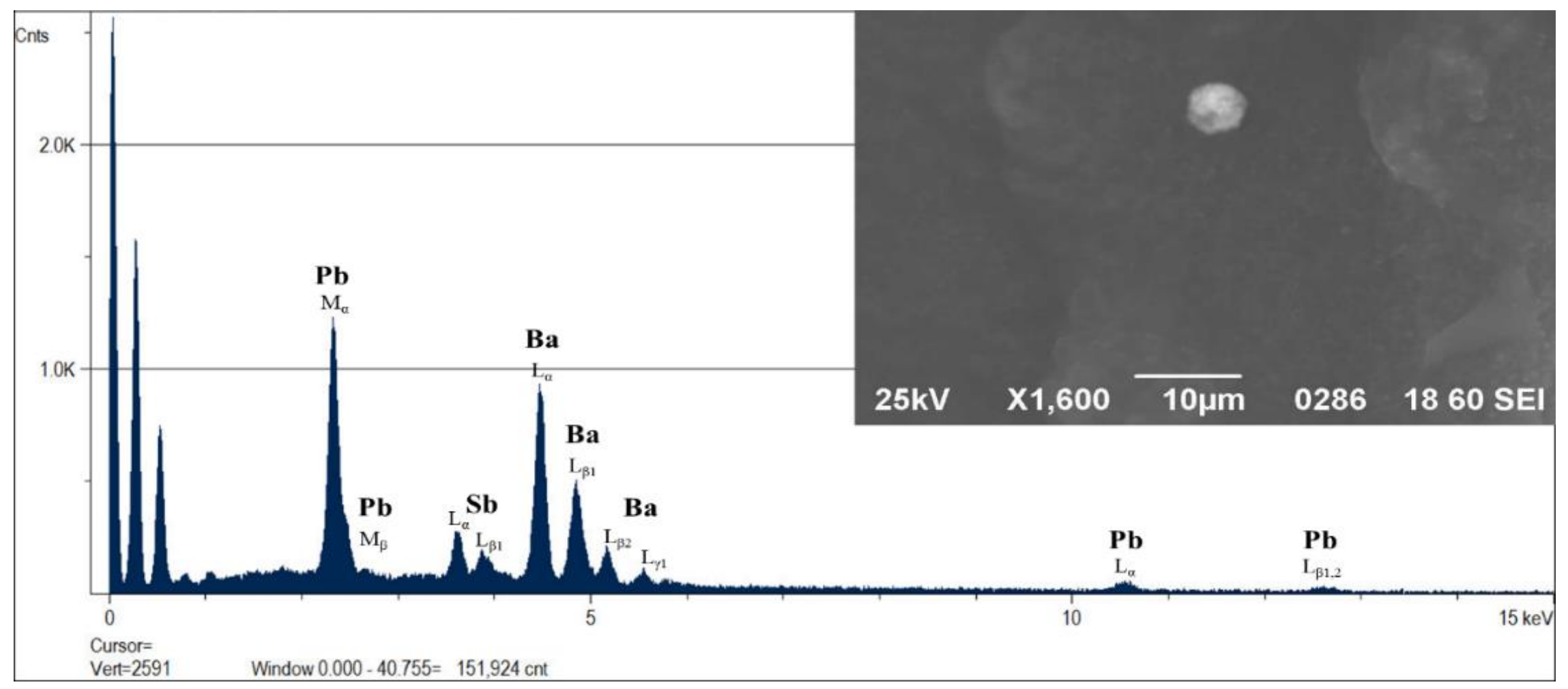

Figure 4: SEM-EDS spectrum and image of the only characteristic GSR particle identified during the confirmation of low-risk background hand samples. 


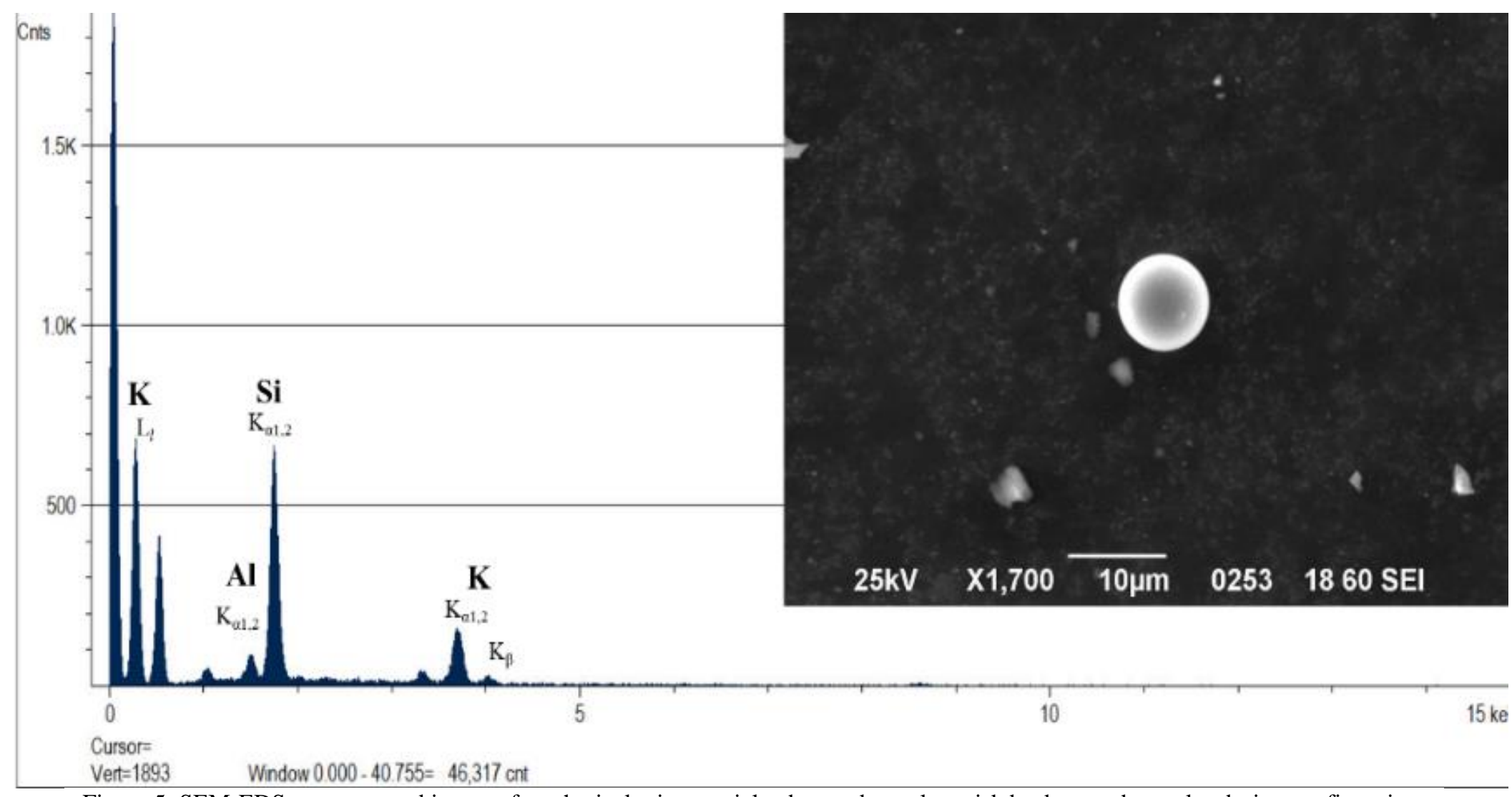

Figure 5: SEM-EDS spectrum and image of a spherical microparticle observed on a low-risk background samples during confirmation. 


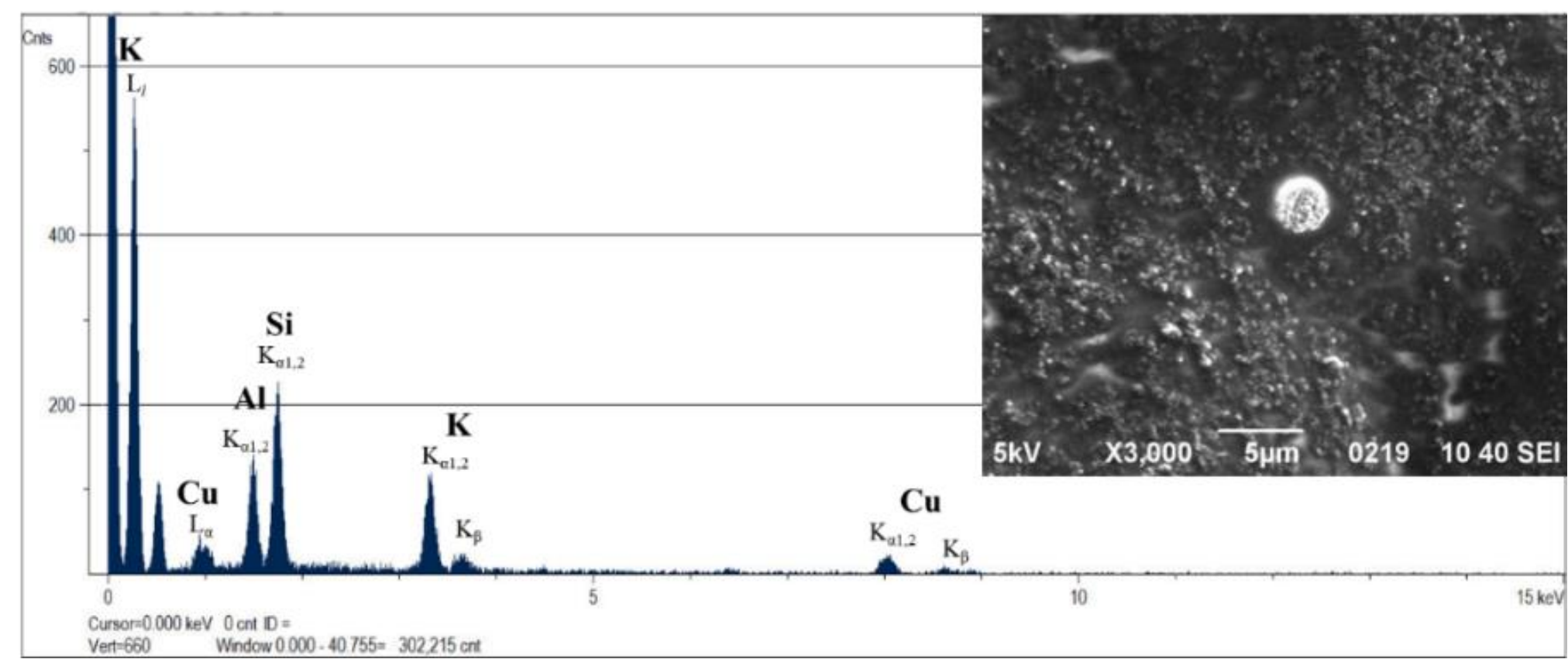

Figure 6: SEM-EDS spectrum and image of a Fiocchi shooter sample observed during the confirmation of the lead-free known shooter samples. 


\section{Leaded Shooter Classes}

Overall

$\mathrm{n}=52$ stubs

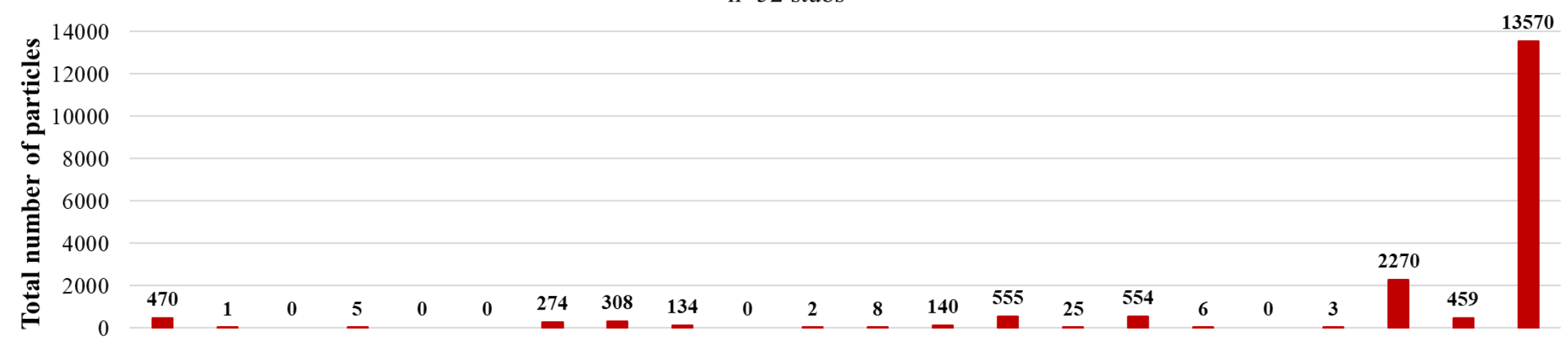

Adjusted y-axis

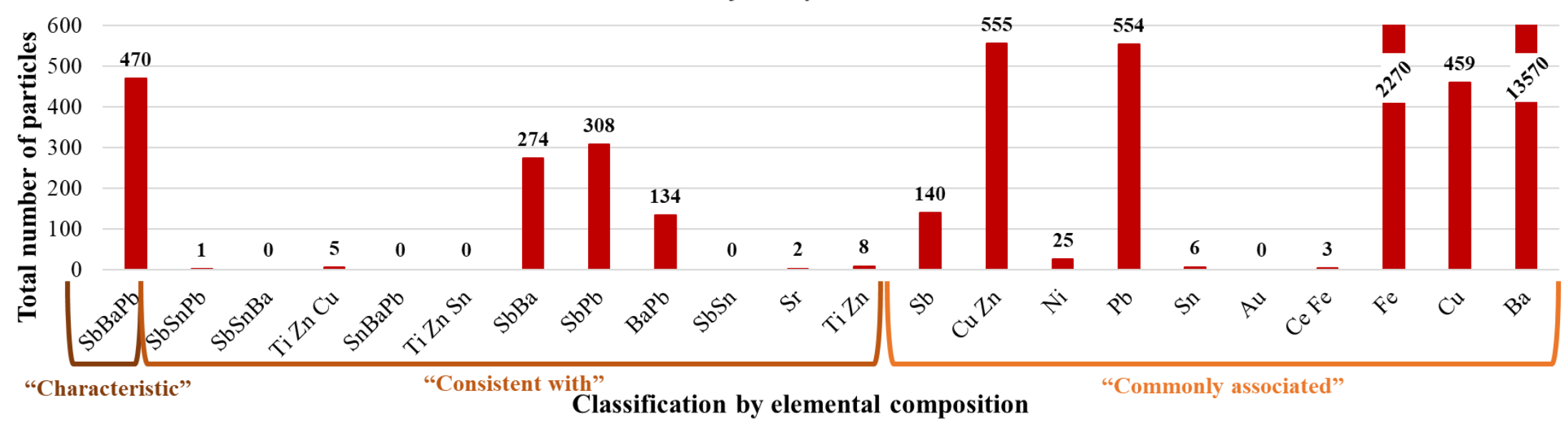

Figure 7: Leaded known shooter population and the classification with rankings for the total number of particles found on all the stubs analyzed $(\mathrm{n}=52)$. 
The other two types of lead-free ammunition analyzed were SYNTECH with only bismuth present as an IGSR element of interest (Figure 10) and Fiocchi with binary particles of copper and potassium, both lighter atomic weight elements (Figure 11). Particles that were considered characteristic were identified with customized elemental combinations based on a combined criterion from the anticipated compositions from the characterized tailor-made IGSR primers and the ASTM standard guidelines. Despite the challenges of only one GSR marker of interest in the SYNTECH ammunition and lighter elements in the Fiocchi ammunition, the automated SEM-EDS discovery for IGSR analysis was able to identify the particles based on these adapted combinations correctly. So, despite the changing elemental composition of modern ammunition, it is possible to identify IGSR by SEM-EDS's current gold standard when using custom-made classification recipes. Studies like this help increasing the body of knowledge on compositions observed in contemporary ammunitions.

Lastly, it is important to note that although morphology and single-particle EDS capabilities are considered one of the SEM-EDS strengths, in authentic specimens, the GSR residues are not predominantly the ideal spheroid/characteristic type ${ }^{59}$ Indeed, we observed that most of the particles created during the discharge were irregular and/or non-characteristic compositions. Demystifying this concept is critical to understand why SEM-EDS is timeconsuming. The automated mapping step requires precisely programmed recipes for identifying those characteristic particles among a large universe of other particles in the stub with less distinctive features. The technological advances make this process conveniently automated, running unattended overnight or while the analyst focuses on other tasks. Once the computerized routine is finalized, the particles flagged by the software must be confirmed by the examiner. In the end, human expertise and judgment are used to form the opinion and draw conclusions. This process delivers accurate results but takes time. Moreover, in the presence of lighter elements formulations, the optimal automated parameters change drastically. The contrast and other variables need to be adjusted accordingly, and some spheroid features are less prominent. 


\section{Lead-free Classes}

Overall

$\mathrm{n}=19$ stubs

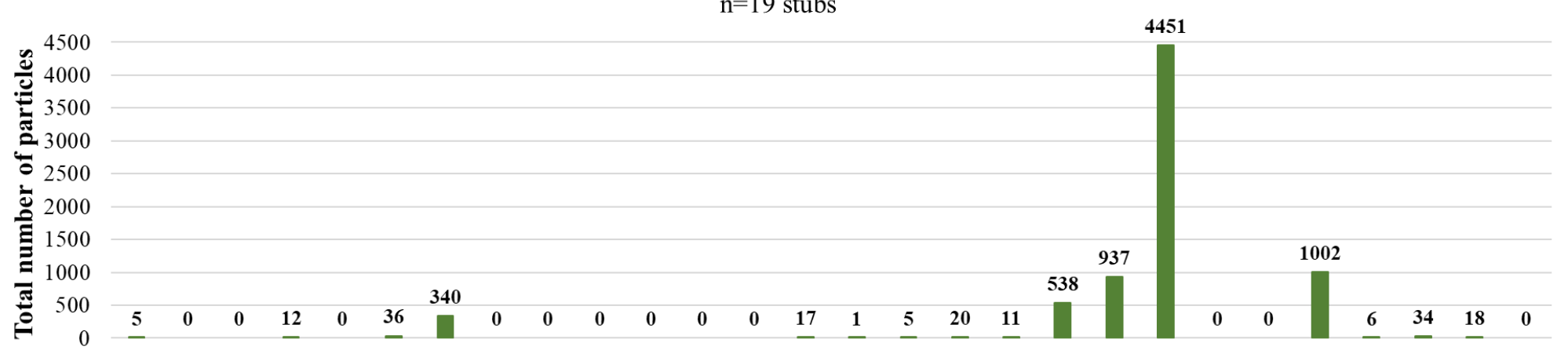

Adjusted y-axis

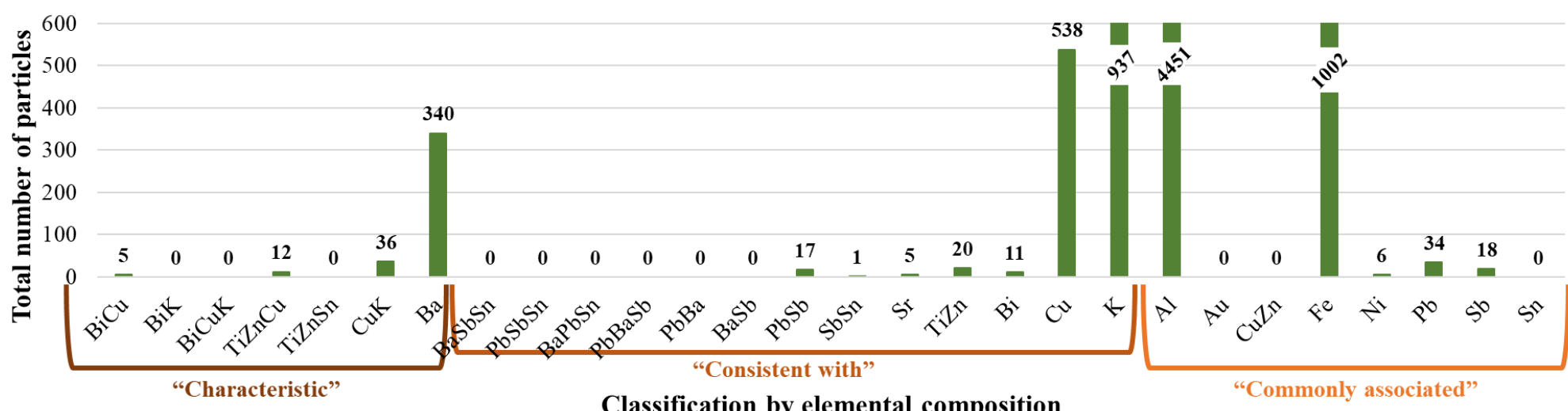

Figure 8: Lead-free known shooter from CCI ammunition and the classification with rankings for the total number of particles found on all the stubs analyzed $(n=19)$. 


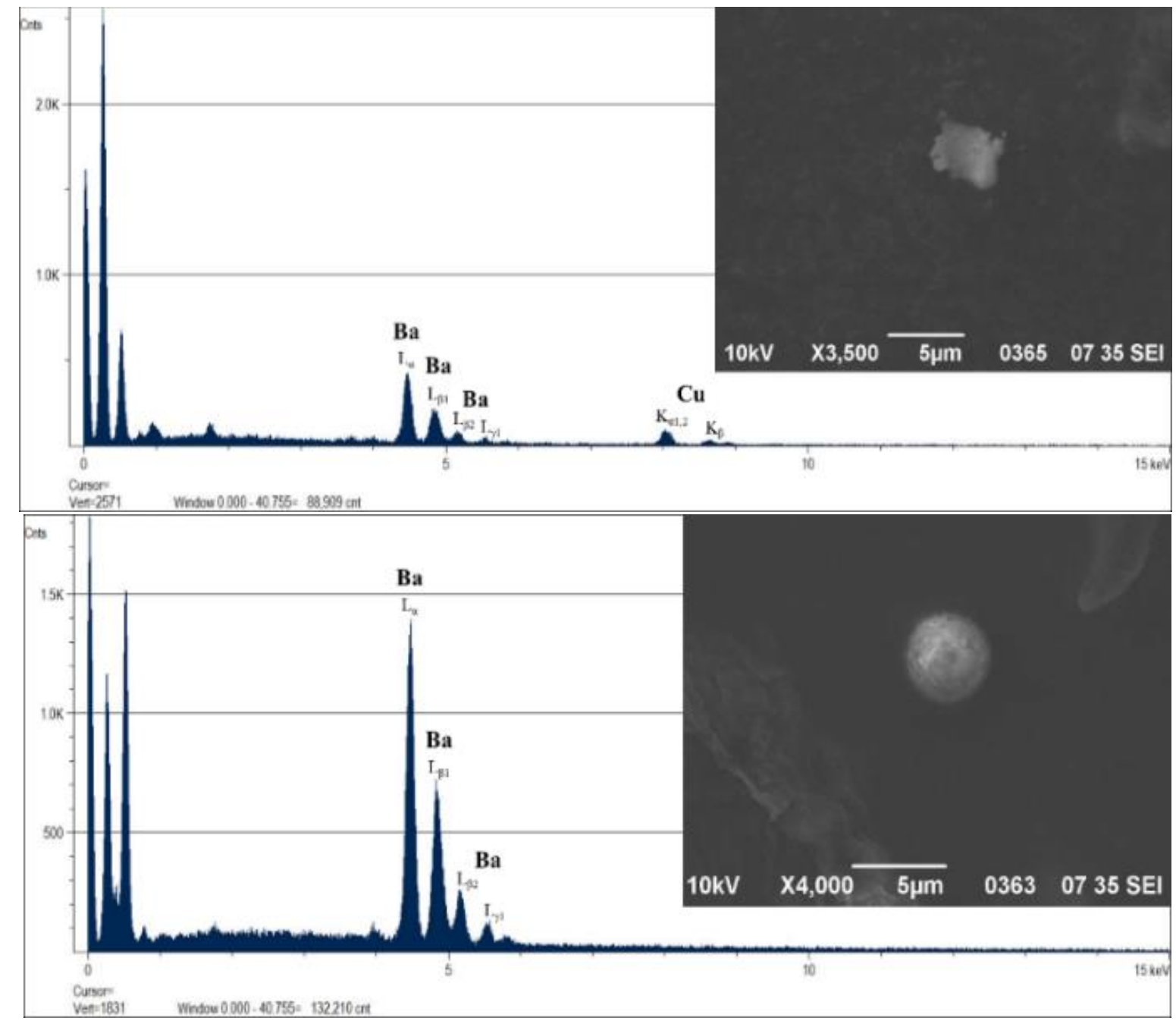

Figure 9: SEM-EDS spectrum and image of irregular (top) and spherical (bottom) CCI GSR particles identified during the confirmation of lead-free known shooter hand samples. 


\section{SYNTECH Lead-free Classes}

Overall

$\mathrm{n}=14$ stubs

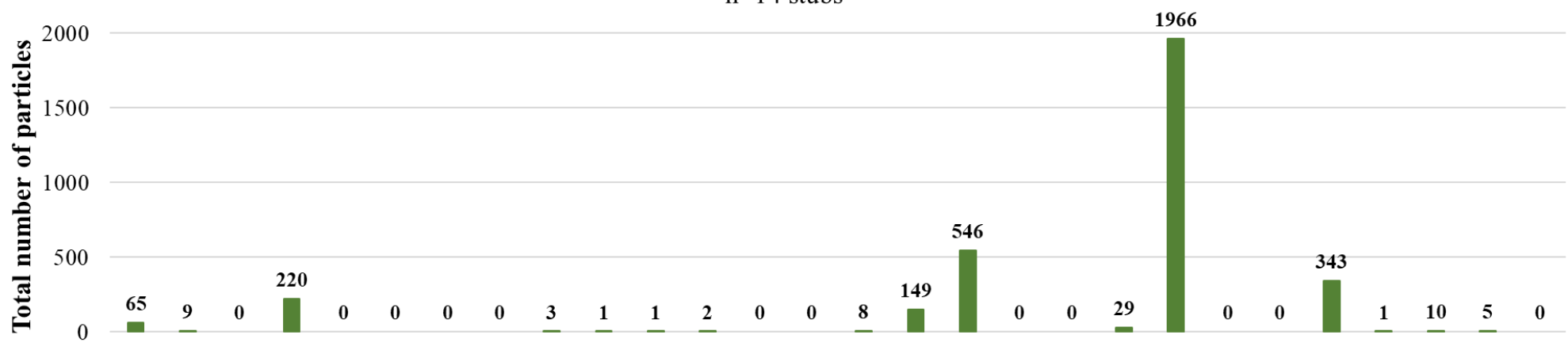

Adjusted y-axis

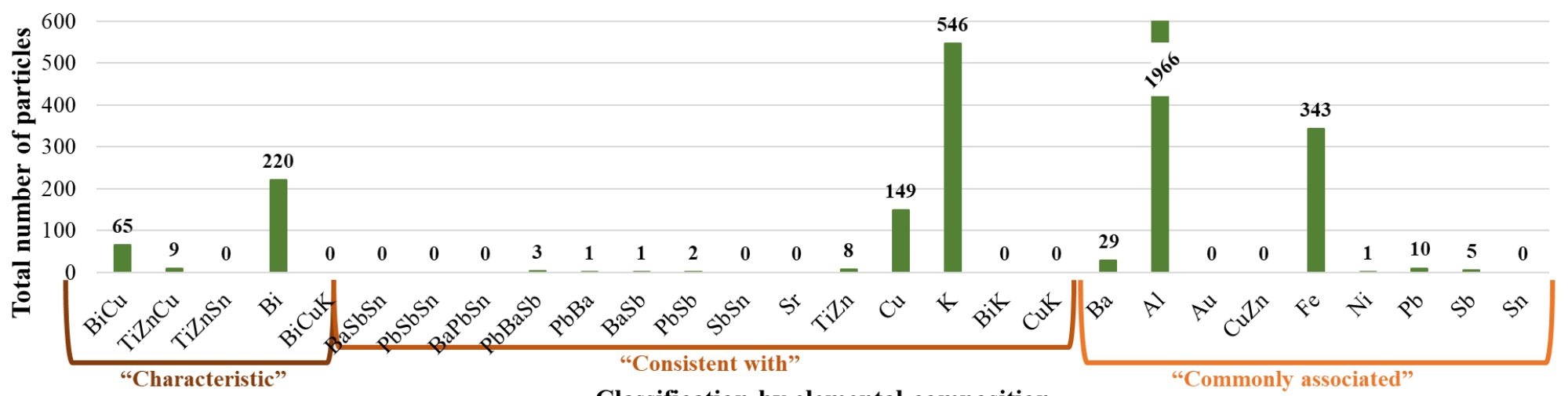

Classification by elemental composition

"Commonly associated"

Figure 10: Lead-free known shooter from SYNTECH ammunition and the classification with rankings for the total number of particles found on all the stubs analyzed $(n=14)$. 


\section{Fiocchi Lead-free Classes}

Overall

$\mathrm{n}=17$ stubs

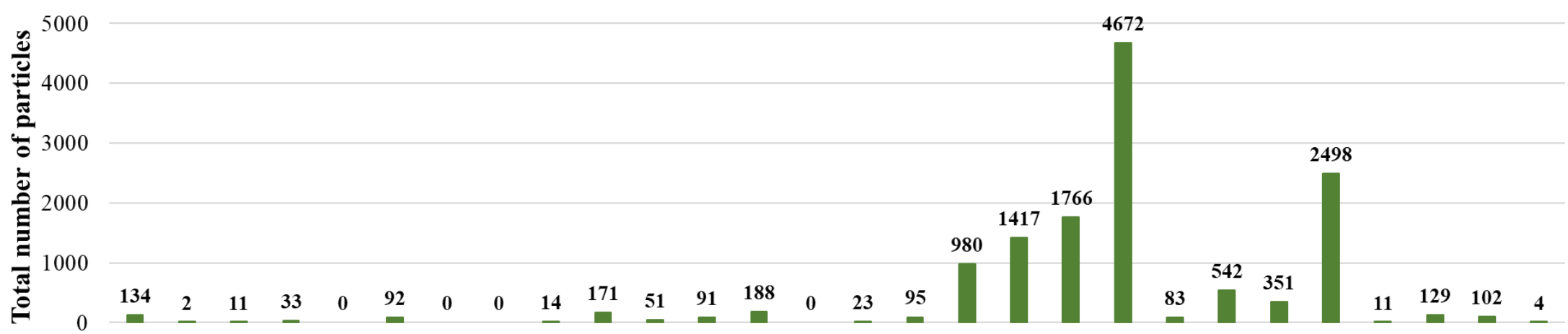

Adjusted y-axis

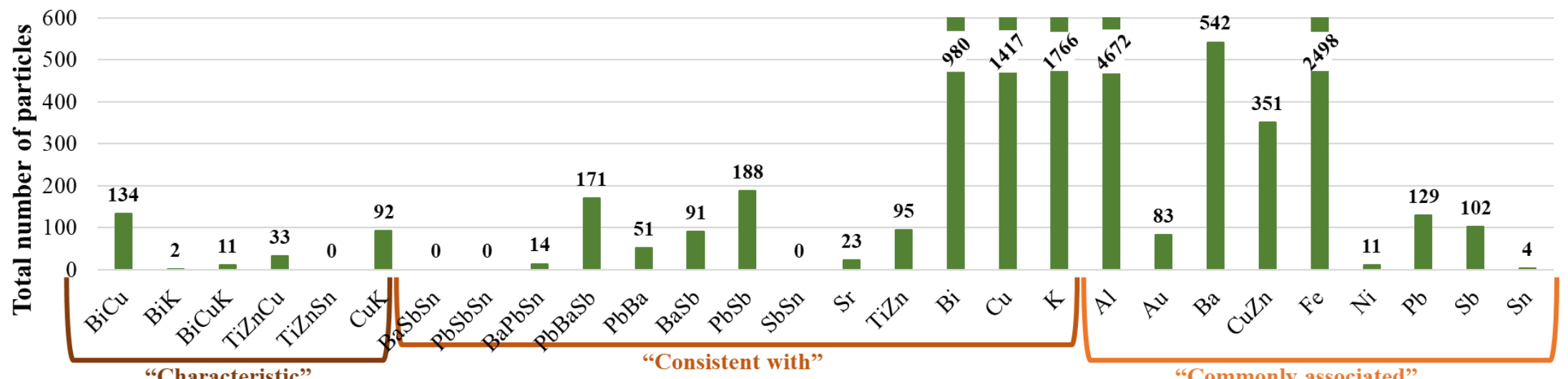

Classification by elemental composition

"Commonly associated"

Figure 11: Lead-free known shooter from Fiocchi ammunition and the classification with rankings for the total number of particles found on all the stubs analyzed $(n=17)$. 
The SEM-EDS findings in this research confirm its validity and numerous advantages. It also highlights the need for modernizing current practice with complementary tools that can assist the practitioners with more overarching case interpretation protocols. For instance, LIBS can help by providing an option of conducting fast screening before SEM-EDS confirmation, otherwise not available. Benefits are three-fold; first, it helps gain knowledge of the expected compositions on the specimens, saving time preparing beforehand the recipe search protocol accordingly. This becomes particularly useful if no conventional $\mathrm{Pb}-\mathrm{Ba}-\mathrm{Pb}$ ammunition was used. Second, it can help to prioritize which specimens need to undergo confirmation, to improve response times, and to reduce backlogs. Finally, due to the speed of analysis, LIBS can be used for alternative surfaces of items that, together with the conventional hand-specimens, can help to answer questions related to transfer and persistence hypothesis, providing more overarching interpretation models. The section below will discuss the feasibility of LIBS for such approaches.

A critical proof of principle in this study was to demonstrate that SEM-EDS GSR detection was possible after LIBS screening. Also, it was important to demonstrate that adding a LIBS step would not pose risk of contamination. Both, the risk for loss or contamination of GSR by incorporating LIBS screening before SEM-EDS was proved to be insignificant. This was demonstrated on a dataset of over 158 samples as discussed before with another 50 high-risk samples in progress.

\subsubsection{LIBS Analysis on the Population Sets}

\subsubsection{Critical Threshold}

The samples collected over the course of this project provided a large data set to evaluate different statistical methods. Data preprocessing cropping the collected spectra to selected regions, perform background subtraction, peak identification, and integration of the peaks of interest, in that order. The integrated peak files were imported into Excel where the signal-to-noise ratio (SNR) was calculated for 45 different emission lines (Table 3). Multiple emission lines were monitored to increase the confidence in the presence of each element. The emission lines were chosen based on elements used in the ASTM 1588-20 standard and the characterized ammunition described in chapter 2. The SNR of the major emission lines were exported and used for further statistical analysis. 
Table 3: List of the monitored emission lines per element.

\begin{tabular}{|c|c|c|c|}
\hline \multicolumn{4}{|c|}{ Monitored Element Emission Lines and their Wavelength (nm) } \\
\hline Al_394.4 & $\mathrm{Cu} \_324.8$ & $\mathrm{Na} \_589.0$ & Si_390.6 \\
\hline Al_396.2 & $\mathrm{Cu}$ _327.4 & Na_589.6 & Sn_284.0 \\
\hline Ba_455.4 & Cu_515.3 & $\mathrm{Na} \_819.5$ & Sn_317.5 \\
\hline Ba_493.4 & Fe_259.9 & Pb_368.3 & $\mathrm{Sn} \_326.2$ \\
\hline Ba_614.2 & $\mathrm{Fe} \_274.7$ & Pb_405.8 & Ti_334.9 \\
\hline Ba_649.7 & $\mathrm{Fe} \_275.6$ & Sb_252.9 & Ti_368.5 \\
\hline Bi_293.8 & K_766.5 & $\mathrm{Sb} \_259.8$ & Ti_375.9 \\
\hline Bi_412.2 & K_769.9 & Sb_287.8 & Ti_376.1 \\
\hline Bi_472.3 & Mg_279.6 & $\mathrm{Sb} \_326.8$ & $\mathrm{Zn} \_328.2$ \\
\hline Ca_315.9 & Mg_280.3 & Si_251.4 & $\mathrm{Zn \_ 334.5}$ \\
\hline Ca_393.4 & Mg_518.4 & Si_288.2 & $\mathrm{Zn} \_481.1$ \\
\hline Ca_396.9 & & & \\
\hline
\end{tabular}

The critical threshold method was the first step in the data analysis; it was used as an exploratory technique to learn about the relevant element variables, examine the performance of the method, understand the variability of the data, and evaluate criteria for a positive and negative threshold. An experimental confidence interval was established at approximately $99.7 \%$ (three standard deviations, assuming normal distribution). A result was considered positive when a SNR was higher than the mean plus three times the standard deviation of the background set (nonshooters) for each element of interest. A negative result corresponded to a SNR below the threshold cutoff. A different threshold was calculated depending on the data set (i.e., OGH or WSJ) according to their respective background sets and relative environmental conditions. All positive and negative results were included to train the prediction methods with real data variability.

The threshold method also allowed pre-processing or filtering of the data, which improves the training of the machine learning algorithms. Due to the random distribution of the GSR particles on a surface of the stub, the laser is not expected to interact with a GSR particle in all the 25 spatial locations measured (25 per stub time 4 stubs: 100 sampled locations for each individual hand-set). Therefore, for each shooter sample, out of the 100 data files, only the discrete spots that detected GSR markers above the threshold were kept. In the case of any false negative results from a shooter sample, only one of the negative spot from the left palm is kept for each sample set. This improves the performance because the algorithm is not being trained with data for shooter samples where the laser did not interact with GSR particles or did not detect GSR. For background samples 
though, all 25 spatial locations were kept for model training purposes, as it is hypothesized that no GSR particles will be present on the backgrounds and if they do, the numbers are critical to establish realistic cut off thresholds.

At the beginning of this study, we started with the collection of only leaded shooter and low-risk background hand samples. The classification of leaded IGSR has been studied extensively and has shown that a particle containing $\mathrm{Pb}-\mathrm{Ba}-\mathrm{Sb}$ is quite uncommon in the background environment as represented by the low-risk background hand samples analyzed by LIBS and SEMEDS. Moreover, in all the GSR specimens generated from the discharge of leaded ammunition, the LIBS method was able to detect particles with the characteristic $\mathrm{Pb}-\mathrm{Ba}-\mathrm{Sb}$ composition. For this reason, classification of the leaded shooter versus low-risk background had an accuracy of 93.5\% (Table4) in this subset. A relatively low critical threshold calculated from the low-risk background samples showed that these elements are not common residues found on people's hand, so the presence of two or more of these elements detected in a single micro-spatial area is indicative of IGSR. The detection of IGSR elements of interest on the hands of individuals who have not discharges a firearm assists in increasing our understanding of the presence of these elements in the environment and differentiate between IGSR and non-IGSR.

The addition of lead-free modern ammunition to the population study has been challenging because of the lack of standard criteria to identify positive GSR profiles. The current ASTM standard for lead-free GSR provides possible elemental combinations and the subsequent classifications. Table 1 in section 1.2 in the introduction provided a summary of the current identification and interpretation scheme. However, the list of elements characteristic of GSR is not exhaustive. For instance, according to the standard, none of the lead-free samples that we analyzed would be considered characteristic of IGSR, and only one type of ammunition collected (INC) would be classified as consistent with GSR. Moreover, none of our sampled lead-free ammunition had the mentioned gadolinium or gallium compositions, indicating once more the dynamic variations in the market. As a result, we developed an in-house scheme for classifying lead-free samples as positive for GSR, using the characterization of the primer-only study of tailor-made IGSR suspensions as a ground truth of its primer composition. A summary of the element(s) considered for a positive GSR using the critical threshold and a categorical approach can be found in Table 5. 
Table 4: Critical threshold results for the leaded shooter $(n=150)$ and low-risk background $(n=110)$ for a subset of the shooter-leaded population by LIBS.

\begin{tabular}{cc}
\hline $\begin{array}{c}\text { Performance } \\
\text { measure }\end{array}$ & $\begin{array}{c}\text { Critical } \\
\text { Threshold }\end{array}$ \\
\hline False positive & $2.0 \%$ \\
False negative & $12.7 \%$ \\
True negative & $98.8 \%$ \\
(Specificity) & \\
True positive & $87.3 \%$ \\
(Sensitivity) & \\
Accuracy & $93.5 \%$
\end{tabular}

Table 5: Summary of the positive calls criteria for the known shooter samples collected. A set was considered positive if the GSR marker combination specified was identified within a single ablation spot.

\begin{tabular}{cc}
\hline Ammunition type & Lead-free GSR markers \\
\hline INC & $\mathrm{Ti}$ and $\mathrm{Zn}$ \\
$\mathrm{SYN}$ & $\mathrm{Bi}$ \\
$\mathrm{FIO}$ & $\mathrm{K}$ or $\mathrm{Cu}$ or both \\
$\mathrm{CCX}$ & $\mathrm{Ba}$ \\
$\mathrm{HEV}$ & $\mathrm{Ba}$ and $\mathrm{Bi}$ \\
NOTE: Leaded ammunition- $\mathrm{Pb}, \mathrm{Ba}$, and $\mathrm{Sb}$. If two or more were \\
present in a single spot, the set was considered positive.
\end{tabular}

When reporting the presence of elements for lead-free primers, multiple factors were considered, and the data was analyzed in different ways using the critical threshold method. Some types of ammunition only have one GSR marker and the threshold for the element is high in the background population. For example, barium and potassium have high background thresholds, 12.2 and 45.2 SNR respectively. When considering the critical threshold, the number of samples determined to be positive is less than if we would use a constant analytical detection cut off of SNR of 3. Some elements of interest present in the samples are not in concentrations high enough to be differentiated from the low-risk background samples used to set the critical thresholds. A summary of these differences can be found in Table 6. This indicates that a critical threshold may 
not be an effective approach for identification of leaded-free GSR. Alternative algorithms may improve the classification efficiency.

Table 6: Summary table of the difference between the number of positive sets depending on the positive criteria set.

\begin{tabular}{cccc}
\hline $\begin{array}{c}\text { Collection date and } \\
\text { ammunition type }\end{array}$ & Total number of sets & $\begin{array}{c}\text { Above the critical } \\
\text { threshold }\end{array}$ & $\begin{array}{c}\text { Above } 3 \text { times the } \\
\text { noise }\end{array}$ \\
\hline 61520_SYN & 15 & 15 & 15 \\
\hline 61520_INC & 15 & 15 & 15 \\
\hline 62220_FIO & 20 & 5 & 20 \\
\hline 62220_CCX & 20 & 20 & 2 \\
\hline 62920_HEV & 2 & 0 & 14 \\
\hline 62920_CCX & 14 & 12 & \\
\hline
\end{tabular}

The 105 high-risk samples collected have been preliminary analyzed by using the critical threshold method, but not yet incorporated into the large population set. From the samples collected, 58 out of 105 have been positive for GSR elements of interest that are used in this study. A complete list of the false positive samples and the elements that were detected can be found in Table 7. The elements detected are indicative of GSR based on the classification criteria developed in our laboratory using the tailor-made $p$ GSR standards to help us better understand the changes in elemental composition of modern ammunition. Numerous samples could be classified as characteristic or consistent with GSR, so further SEM-EDS analysis is imperative to understanding the results. Once completed, a more through discussion of the results will be added and the highrisk population will be incorporated into the performance rates and machine learning algorithms. 
Table 7: List of all the positive samples from the high-risk population and the elements observed (false positive). While $\mathrm{Cu}$ is not typically used to identify GSR, it is a major element in lead-free FIO and was monitored in this study. These samples are awaiting confirmation by SEM-EDS. Legend; FRA is firearm/impression research laboratory, AGL is farm and greenhouse, MEC is mechanic, and POL is police/station.

\begin{tabular}{|c|c|c|c|}
\hline Positive Stubs & $\begin{array}{l}\text { Positive } \\
\text { elements }\end{array}$ & Positive Stubs & $\begin{array}{l}\text { Positive } \\
\text { elements }\end{array}$ \\
\hline 030921_013_BGH_FRA_HR_LH & $\mathrm{Sb}, \mathrm{Pb}$ & 033021_056_BGH_MEC_HR_both & $\mathrm{Sb}, \mathrm{Ba}, \mathrm{Ti}$ \\
\hline 030921_014_BGH_FRA_HR_LH & $\mathrm{Sb}, \mathrm{Pb}, \mathrm{Cu}, \mathrm{Ti}$ & 033021_057_BGH_MEC_HR_both & $\mathrm{Sb}, \mathrm{Ba}, \mathrm{Ti}$ \\
\hline 030921_015_BGH_FRA_HR_LH & $\mathrm{Sb}, \mathrm{Pb}, \mathrm{Ba}, \mathrm{Cu}$ & 033021_058_BGH_MEC_HR_both & $\mathrm{Sb}, \mathrm{Ba}, \mathrm{Ti}$ \\
\hline 030921_016_BGH_FRA_HR_LH & $\mathrm{Cu}$ & 033021_059_BGH_MEC_HR_both & $\mathrm{Sb}, \mathrm{Ba}, \mathrm{Ti}$ \\
\hline 030921_019_BGH_FRA_HR_LH & $\mathrm{Cu}$ & 033021_060_BGH_MEC_HR_both & $\mathrm{Sb}, \mathrm{Ba}, \mathrm{Ti}$ \\
\hline 031021_021_BGH_FRA_HR_LH & $\mathrm{Sb}, \mathrm{Pb}, \mathrm{Ba}, \mathrm{Ti}$ & 033021_061_BGH_MEC_HR_both & $\mathrm{Sb}, \mathrm{Ba}, \mathrm{Ti}$ \\
\hline 031021_022_BGH_FRA_HR_LH & $\mathrm{Cu}$ & 033021_062_BGH_MEC_HR_both & $\mathrm{Sb}, \mathrm{Ba}, \mathrm{Ti}$ \\
\hline 031021_023_BGH_FRA_HR_RH & $\mathrm{Sb}, \mathrm{Pb}$ & 033021_063_BGH_MEC_HR_both & $\mathrm{Sb}, \mathrm{Ba}, \mathrm{Ti}$ \\
\hline 031021_026_BGH_FRA_HR_LH & $\mathrm{Cu}$ & 033021_065_BGH_MEC_HR_RH & $\mathrm{Sb}$ \\
\hline 031021_027_BGH_FRA_HR_LH & $\mathrm{Sb}, \mathrm{Pb}, \mathrm{Ba}, \mathrm{Cu}$ & 033021_066_BGH_AGL_HR_RH & $\mathrm{Sb}, \mathrm{Pb}, \mathrm{Ti}$ \\
\hline 031021_028_BGH_FRA_HR_LH & $\mathrm{Sb}, \mathrm{Pb}, \mathrm{Ba}$ & 033021_068_BGH_AGL_HR_LH & $\mathrm{Sb}, \mathrm{Pb}, \mathrm{Ba}$ \\
\hline 031021_031_BGH_FRA_HR_LH & $\mathrm{Cu}$ & 033021_069_BGH_AGL_HR_RH & $\mathrm{Sb}, \mathrm{Pb}$ \\
\hline 031821_033_BGH_AGL_HR_LH & $\mathrm{Sb}, \mathrm{Ba}$ & 033021_070_BGH_AGL_HR_RH & $\mathrm{Sb}, \mathrm{Pb}, \mathrm{Ba}$ \\
\hline 031821_034_BGH_AGL_HR_LH & $\mathrm{Sb}, \mathrm{Ba}$ & 033021_071_BGH_AGL_HR_LH & $\mathrm{Sb}, \mathrm{Pb}$ \\
\hline 031821_036_BGH_AGL_HR_RH & $\mathrm{Sb}, \mathrm{Ba}$ & 033121_074_BGH_POL_HR_LH & $\mathrm{Cu}$ \\
\hline 031821_037_BGH_AGL_HR_RH & $\mathrm{Sb}, \mathrm{Ba}$ & 033121_075_BGH_POL_HR_both & $\mathrm{Sb}, \mathrm{Ba}, \mathrm{Cu}$ \\
\hline 031821_040_BGH_AGL_HR_RH & $\mathrm{Sb}, \mathrm{Ba}$ & 033121_076_BGH_POL_HR_LH & $\mathrm{Sb}, \mathrm{Pb}, \mathrm{Ba}, \mathrm{Ti}$ \\
\hline 031821_041_BGH_AGL_HR_LH & $\mathrm{Sb}, \mathrm{Ba}$ & 033121_078_BGH_POL_HR_LH & $\mathrm{Cu}$ \\
\hline 031821_043_BGH_AGL_HR_RH & $\mathrm{Sb}, \mathrm{Ba}$ & 033121_080_BGH_POL_HR_RH & $\mathrm{Cu}$ \\
\hline 031821_045_BGH_AGL_HR_LH & $\mathrm{Sb}, \mathrm{Ba}$ & 033121_083_BGH_POL_HR_RH & $\mathrm{Cu}, \mathrm{Ti}$ \\
\hline 033021_047_BGH_MEC_HR_both & $\mathrm{Sb}, \mathrm{Ba}, \mathrm{Ti}$ & 033121_084_BGH_POL_HR_LH & $\mathrm{Sb}, \mathrm{Pb}, \mathrm{Ti}$ \\
\hline 033021_048_BGH_MEC_HR_both & $\mathrm{Sb}, \mathrm{Ba}, \mathrm{Ti}$ & 033121_090_BGH_POL_HR_RH & $\mathrm{Cu}$ \\
\hline 033021_049_BGH_MEC_HR_both & $\mathrm{Sb}, \mathrm{Ba}, \mathrm{Ti}$ & 033121_093_BGH_POL_HR_LH & $\mathrm{Sb}, \mathrm{Pb}, \mathrm{Ba}$ \\
\hline 033021_050_BGH_MEC_HR_both & $\mathrm{Sb}, \mathrm{Ba}, \mathrm{Ti}$ & 033121_095_BGH_POL_HR_LH & $\mathrm{Cu}$ \\
\hline 033021_051_BGH_MEC_HR_both & $\mathrm{Sb}, \mathrm{Ba}, \mathrm{Ti}$ & 033121_095_BGH_POL_HR_both & $\mathrm{Sb}, \mathrm{Pb}, \mathrm{Ba}$ \\
\hline 033021_052_BGH_MEC_HR_both & $\mathrm{Sb}, \mathrm{Ba}, \mathrm{Ti}$ & 033121_098_BGH_POL_HR_LH & $\mathrm{Sb}, \mathrm{Pb}, \mathrm{Ti}$ \\
\hline 033021_053_BGH_MEC_HR_both & $\mathrm{Sb}, \mathrm{Ba}, \mathrm{Ti}$ & 033121_099_BGH_POL_HR_RH & $\mathrm{Cu}, \mathrm{Ti}$ \\
\hline 033021_054_BGH_MEC_HR_both & $\mathrm{Sb}, \mathrm{Ba}, \mathrm{Ti}$ & 033121_103_BGH_POL_HR_both & $\mathrm{Sb}, \mathrm{Pb}, \mathrm{Ba}$ \\
\hline 033021_055_BGH_MEC_HR_both & $\mathrm{Sb}, \mathrm{Ba}, \mathrm{Ti}$ & 033121_105_BGH_POL_HR_RH & $\mathrm{Sb}, \mathrm{Pb}, \mathrm{Ba}$ \\
\hline
\end{tabular}




\subsubsection{Machine Learning Algorithms for Classification of GSR and Non-GSR Residues}

After the critical threshold was used for classification and exploratory purposes, we moved onto machine learning algorithms. Naïve Bayes, logistic regression, and a neural network were applied to the data to classify the samples into either GSR being present (known shooter populations) or GSR not being present (low-risk background population). The algorithms were previously tested during the development and validation of the micro-spatial LIBS method with promising results. ${ }^{16}$ These algorithms were chosen primarily since the data is classified based on the probability of it belonging to a specific group based on their spectral profiles, and the probabilities are critical for Bayesian interpretation and probabilistic assessment discussed in the next section. Besides the probabilities, these algorithms can be trained with ground-truth data sets like the one we collected and assist in automatic classification of the data with little user input, therefore providing assessment of the sample faster, more objectively, and requires less time for the analyst to interpret the results. Also, the algorithms can handle multiple variables and classification outputs, which is beneficial to the large population with multiple variables being considered and the large amount of data being collected per sample ( 25 spectra for every stub, resulting in up to 100 spectra for shooter and 50 spectra for non-shooter sets). Machine learning algorithms are quite robust and can analyze thousands of lines of data in the matter of seconds.

When machine learning was applied to the data, eight elements and two different wavelengths for each element were used. The selection of elements was based on the ground truth from previous primer characterization and the recommendations of ASTM. Additional elements are monitored during LIBS, but only the elements listed in Table 8 were used when training the machine learning algorithms. The components included the leaded elements of interest $(\mathrm{Pb}, \mathrm{Ba}$, and $\mathrm{Sb}$ ) and additional elements to assist in classifying lead-free ammunition ( $\mathrm{Cu}, \mathrm{Ti}, \mathrm{Zn}, \mathrm{Bi}, \mathrm{K})$. The additional variables also increased the overall accuracy of the classification methods, from accuracies better than $\sim 80 \%$ when only leaded elements are used to better than $\sim 90 \%$ with the additional elements are considered on potential elemental profiles. The false negative rate greatly decreased, but the false positive rate did increase, but not by as much (Table 9). While this increase in the false positive rate is not ideal, it is manageable for a screening test unlike a high false negative rate due to the following confirmatory stage. Any positive sample will be further analyzed by confirmatory SEM-EDS, while negative samples might not be if this technique is used as a tool to increase efficiency in a crime laboratory, make informed decisions and triage, and decrease the 
number of samples being analyzed. The comparison of the accuracy using the different number of elements is displayed in Table 9.

Table 8: Element and monitored wavelength $(\mathrm{nm})$ used in the training of the machine learning algorithms and separated by the type of the ammunition the elements assist in classifying. * Mixed: leaded and lead-free group elements in addition to the elements listed here

\begin{tabular}{ccccccc}
\hline Ammunition type & \multicolumn{5}{c}{ Element and monitored wavelength (nm) } \\
\hline Leaded & $\mathrm{Pb} \mathrm{405.8}$ & $\mathrm{Pb} \mathrm{368.3}$ & $\mathrm{Sb} \mathrm{259.8}$ & $\mathrm{Sb} \mathrm{252.8}$ & $\mathrm{Ba} \mathrm{455.4}$ & Ba 493.4 \\
\hline Mixed* $^{*}$ & $\mathrm{Cu} \mathrm{327.4}$ & $\mathrm{Cu} \mathrm{324.7}$ & Ti 334.9 & Ti 376.1 & Zn 334.5 & Zn 481.0 \\
\hline Lead-free & Bi 293.8 & Bi 472.2 & K 766.5 & K 769.9 & & \\
\hline
\end{tabular}

Table 9: Comparison of performance rates with the increase in the number of variables (elements and wavelengths) monitored to assist in the classification of modern, lead-free ammunition (350 low-risk backgrounds, 200 leaded shooters, 100 lead-free shooters, and 220 mixed shooters).

\begin{tabular}{ccccccccc}
\hline Data input & \multicolumn{4}{c}{ Three leaded elements of interest } & \multicolumn{3}{c}{ Eight leaded/ lead-free elements of interest } \\
\hline $\begin{array}{c}\text { Performance } \\
\text { measure (\%) }\end{array}$ & $\begin{array}{c}\text { Critical } \\
\text { Threshold }\end{array}$ & $\begin{array}{c}\text { Naïve } \\
\text { Bayes }\end{array}$ & $\begin{array}{c}\text { Logistic } \\
\text { Regression }\end{array}$ & $\begin{array}{c}\text { Neural } \\
\text { Networks }\end{array}$ & $\begin{array}{c}\text { Critical } \\
\text { Threshold }\end{array}$ & $\begin{array}{c}\text { Naïve } \\
\text { Bayes }\end{array}$ & $\begin{array}{c}\text { Logistic } \\
\text { Regression }\end{array}$ & $\begin{array}{c}\text { Neural } \\
\text { Networks }\end{array}$ \\
\hline False positive & 3.0 & 3.0 & 3.7 & 5.3 & 0.0 & 8.7 & 25.2 & 26.1 \\
\hline $\begin{array}{c}\text { False } \\
\text { negative }\end{array}$ & 36.0 & 50.9 & 58.3 & 37.1 & 8.1 & 10.0 & 2.2 & 2.0 \\
$\begin{array}{c}\text { True } \\
\text { negative }\end{array}$ & 97.0 & 97.0 & 96.3 & 94.7 & 100.0 & 91.3 & 74.8 & 73.9 \\
\hline $\begin{array}{c}\text { True positive } \\
\text { (Sensitivity) }\end{array}$ & 64.0 & 49.1 & 41.7 & 62.9 & 91.9 & 90.0 & 97.8 & 98.0 \\
\hline \begin{tabular}{c} 
Accuracy \\
\hline Sc.
\end{tabular} & 80.5 & 82.2 & 79.4 & 84.8 & 95.2 & 90.2 & 95.6 & 95.8 \\
\hline
\end{tabular}

\subsubsection{Probabilistic Interpretation of the Populations}

Once comparison of the different classification methods was completed, then probabilistic interpretation of the populations was conducted. We chose to only proceed with logistic regression and the neural network. Naïve Bayes performed well but had an accuracy $~ 5 \%$ lower than all the other classification techniques. In addition, the assumption of independence between variables might not be true when considering GSR and its formulations. The identification of $\mathrm{Pb}, \mathrm{Ba}$, and $\mathrm{Sb}$ are tied to the initiator, oxidizer, and fuel respectively, all of which need to be present to identify 
leaded IGSR, with the same being true of lead-free but with different initial compounds. Still, the probabilities from the other two algorithms could be applied to populations using Bayesian statistics and reasoning, which can provide a logical and robust framework by providing a more impartial evidence interpretation using the assessment of the two mutually exclusive and exhaustive hypothesis $\left(\mathrm{H}_{p}\right.$ and $\left.\mathrm{H}_{d}\right) .{ }^{35,60}$ At this stage in the study, we are only able to speak to the chemical and morphological information in the population, so we are assessing the evidence at the source level with our two hypothesis being that the trace is GSR from a firearm discharge $\left(\mathrm{H}_{p}\right)$ or the trace is from another source other than GSR.

When analyzing any forensic evidence, there is a level of experience and subjectivity involved. ${ }^{33}$ Bayesian frameworks provide a powerful approach to evidence interpretation that blends the analytical results with contextual information to update beliefs. At the source level, we want to assess the probability of observing the evidence (chemical profile) given the trace came from a firearm discharge $\left(\mathrm{P}\left[\mathrm{E} / \mathrm{H}_{p}\right]\right)$ versus the probability of the evidence given the trace came from an non-related environmental source or is not GSR $\left(\mathrm{P}\left[\mathrm{E} / \mathrm{H}_{d}\right]\right)$. This can be numerically assessed using the likelihood ratio and considering the probability of the evidence as a ratio using the two hypotheses. ${ }^{47}$ From this calculation, we can speak to the likelihood ratio for the evidence analyzed and use the ground truth knowledge $\left(\mathrm{H}_{p}\right.$ true, or $\mathrm{H}_{d}$ true)to determine the distribution of the likelihood ratio values for the different populations. Currently, we can only speak to the likelihood ratio at the source level, but this can also be applied to future work at the activity and this research can serve as a foundation.

In order to evaluate the likelihood ratio distributions of the different populations (Equation 1 and 2), histograms of the different likelihood ratio calculations were created to visualize the likelihood ratios and separation between the populations when $\mathrm{H}_{p}$ or $\mathrm{H}_{d}$ are true. Histograms provide the frequency at which specific likelihood ratios were observed and places them into bins to see the distribution of the data considering a given hypothesis. The taller a bin, the more that specific likelihood ratio was observed. Also, the $\log 10$ of the likelihood ratio was used to better visualize the data and scale the data to a more interpretable number where negative values tend to support $\mathrm{H}_{d}$ and positive values support $\mathrm{H}_{p}{ }^{46}$ Figure 12 shows a comparison of the leaded shooter and low-risk non-shooter population, while also comparing the differences between the likelihood ratio distributions of the two classification algorithms. Both distributions show support for $\mathrm{H}_{p}$, or $\mathrm{H}_{1}$, when the $\log 10$ likelihood ratio is above zero, and the opposite for $\mathrm{H}_{d}$, or $\mathrm{H}_{2}$, and the area of 
overlap can be connected to the discriminating power. The more overlap between the histograms, the less discrimination power is observed.

There is also a clear difference between the likelihood ratios of the two algorithms. Logistic regression likelihood ratios for the shooter set have a large spike at around log LR of 10, but the neural network has an evenly spread distribution between 5 and 10. Same for the low-risk nonshooter where more of the data for logistic regression clusters around -2.5, but the neural network is more spread between -5 and 0 . There is also less overlap between the two distributions when using $\mathrm{NN}$, demonstrating that the neural network has a higher discriminating power. It is worth nothing, than in the machine learning algorithm outputs some probabilities smaller than $10^{-10}$ were observed for the non-GSR group. In such cases, unrealistically large LR were observed for $\mathrm{H}_{1}$. Moreover, the computer precision is often limited to 10 decimal places. Therefore, a decision was made to truncate the probability values to a minimum of $10^{-10}$ before estimating the LRs. This will also help later when calibration LR methods would be applied.

Another way to visualize and empirically measure the performance of likelihood ratios is in the form of Tippet plots. The plots are cumulative versions of the histograms and represent the cumulative proportion of likelihood ratio values. ${ }^{46}$ In one graph, we can observe both likelihood ratio values for when $\mathrm{H}_{p} / \mathrm{H}_{1}$ and $\mathrm{H}_{d} / \mathrm{H}_{2}$ is true and evaluate the rate of misleading evidence at the same time. Where the line plotting the likelihood ratio of the $\mathrm{H}_{p} / \mathrm{H}_{1}$ crosses the dotted line at $\log$ LR 0 , the integrated area between the line and dotted line at the top represents the rate of misleading evidence for that hypothesis, or also called the false negative rate. The opposing hypothesis is where the line plotting the likelihood ratio of the $\mathrm{H}_{d} / \mathrm{H}_{2}$ crosses the dotted line at 0 , the integrated area between the line and dotted line at the bottom is the rate of misleading evidence for that hypothesis, or also called the false positive rate. Figure 13 are Tippet plots for the same histograms shown in Figure 12. As with the histograms, the sharp drop on the Tippet plot for logistic regression for $\mathrm{H}_{1}$ correlates to the sharp spike in frequency of the likelihood ratios at 10 . Interestingly, the Tippet plots show that logistic regression had lower false negative rates, but the neural network has lower false negative rates, all be it both were very small in either (Logistic regression false negative $0.41 \%$, false positives $1.85 \%$; neural network false negative $0.56 \%$, false positive $1.23 \%$ ). The gap between the two lines is also smaller for the neural network, once again confirming a higher discriminating power of the algorithm and subsequent likelihood ratios. 

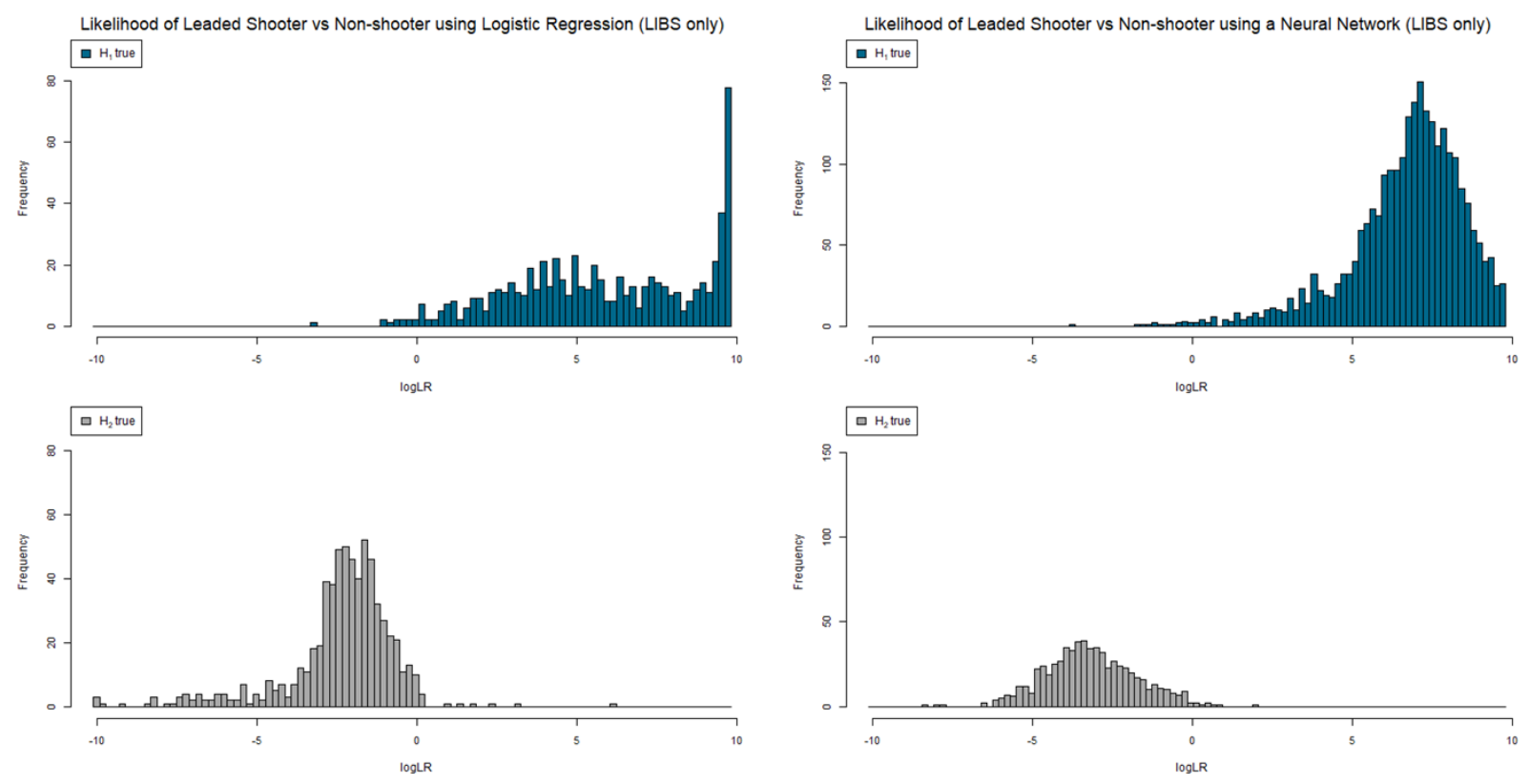

Figure 12: Histograms displaying the distribution of likelihood ratio calculated from logistic regression (left) and a neural network (right). The likelihood ratio 1 equation was used for calculation. $\mathrm{H}_{1}$ is the prosecutor's hypothesis and $\mathrm{H}_{2}$ is the defense hypothesis.

Since our data could not be simply modeled using a parametric model, we applied a Kernel Density Function (KDF) to the histogram distributions to model this data using an estimation. The end result is a continuous curve for the distributions similar in shape to the histograms and displays both distributions in the same graph. Figure 14 is an example of all histograms and corresponding KDF using the neural network outputs for the likelihood ratio calculations (equation 1) for the three different shooter populations (leaded, lead-free and mixed) and the one likelihood ratio calculation (equation 2) using all shooter populations combined. The differences in the distribution of $\mathrm{Hp} / \mathrm{H}_{1}$ likelihood ratios and the relative peak heights is due to the differences in the number of data points and samples within each population considered. Nonetheless, the histograms and KDF distributions are comparable and provide a continuous curve model to apply to additional likelihood ratio calibration in the future. KDF curves provide the likelihood ratio at each point on the curve.

Another interesting observation is the differences in the shapes and distributions of the populations analyzed and likelihood ratio calculation equation. As expected, the leaded versus low-risk background has the least amount of overlap between the two. The likelihood ratio of a non-shooter sample was around -6 to 0 and a leaded shooter around 2 to 12 . The mixed shooter set had the least separation, a non-shooter likelihood ratio from -4 to 2 , and a mixed shooter likelihood 
ratio -1 to 5 with a left skew to it. The lead-free distribution for the non-shooter was comparable to the leaded distribution for non-shooters, but the likelihood ratios were spread between 1 and 10 . There is also humps in the distribution, possibly due to a larger variety of possible GSR elemental profiles of particles of interest contributing to the probability and subsequent likelihood ratio. The last interesting observation is the distribution of the likelihood ratio using equation 2 where all three shooter populations are considered together. We can see two clearly defined peaks in the data, one for the mixed shooter and one for the leaded shooter, with lead-free falling in either area. The distribution for the non-shooter likelihood ratio is also from 0 to 12 , instead of the lower values seen in the other distributions.
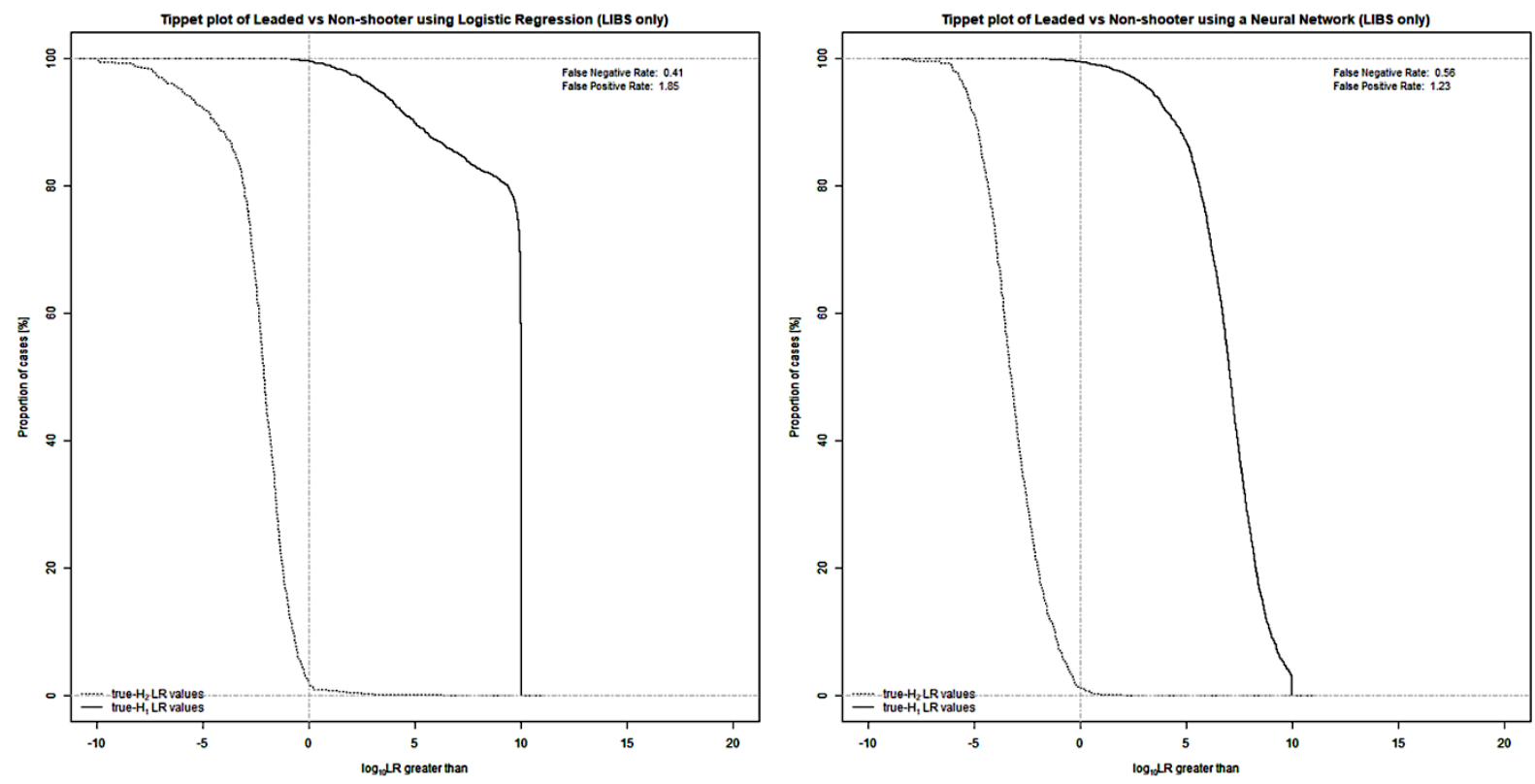

Figure 13: Tippet plots displaying the proportion of misleading cases from logistic regression (left) and a neural network (right). The likelihood ratio 1 equation was used for calculation. $\mathrm{H}_{1}$ is the prosecutor's hypothesis and $\mathrm{H}_{2}$ is the defense hypothesis. Where the line plotting the likelihood ratio of the $\mathrm{H}_{p} / \mathrm{H}_{1}$ crosses the dotted line at log LR 0 , the integrated area between the line and dotted line at the top is the rate of misleading evidence for that hypothesis, or also called the false negative rate. The opposing hypothesis is where the line plotting the likelihood ratio of the $\mathrm{H}_{d} / \mathrm{H}_{2}$ crosses the dotted line at 0 , the integrated area between the line and dotted line at the bottom is the rate of misleading evidence for that hypothesis, or also called the false positive rate.

The results presented here will greatly assist GSR interpretation and practitioners in the future. Understand the distribution of likelihood ratios using the large number of samples in this population study (>2,900 stubs) provides a comparison point for likelihood ratios calculated in the future as a proxy to estimate the weight of the evidence. This studied demonstrated that non-GSR 
samples tend in fact to have lower likelihood ratios $(\log \mathrm{LR}<0)$ and true GSR samples have a higher likelihood ratio $(\log L R>0)$. Our data shows that the more distinctive the elemental profiles and presence of characteristic particles in a sample, the larger the LR, demonstrating LR can serve to inform the uncertainty in reporting GSR on an item of interest.

The minimal histogram overlaps, and the outputs of the tippet plots provided a preliminary assessment of the LR in terms of the distribution of LR values within populations, their discrimination power, and misleading rates. Nonetheless, we believe that LR above $10^{6}$ may be still unrealistic and could provide a misleading certainty belief to the end-users. Therefore, the next step in this study is to explore calibration algorithms to calibrate the LRs, as successfully applied to other forensic disciplines. ${ }^{46,61}$ Post hoc calibration steps can aid in providing more realistic LR while testing the robustness and performance of the LR system. Several approaches have been proposed to evaluate the accuracy of the LR system, such as the use of empirical cross entropy (ECE), or empirical upper and lower bound method, (ULB) to mention some. ${ }^{57,61,62}$

The ULB method uses a known dataset to calibrate the LR by fitting empirically density models to the $\log _{10}$ LRs under Hp and Hd. Then, an independent dataset, or a subset using leaveone-out cross-validation procedure, is selected to confirm the transformed LRs are well calibrated. Using the method reported by Van Es et al, The KDF applied to the histograms mimics the distribution of the original calculated $\log 10$ likelihood ratios. From the new KDF, we can calculate a new likelihood by dividing the likelihood of $\mathrm{H}_{1}$ by $\mathrm{H}_{2}$ at each $\mathrm{x}$ value, which is the newly calibrated likelihood ratio. ${ }^{57}$ The results of the calibrated LR will be compared to the original likelihood ratios calculated by plotting the new calibrated likelihood ratio to the old likelihood ratios to determine if the values are close to an identity relation. ${ }^{57}$ Calibration of the likelihood ratios ensures that the likelihood ratios calculated do not lead to misleading evidence in the form of likelihood ratios that are unrealistically too large or too small. ${ }^{61} \mathrm{~A}$ ill-calibrated likelihood ratio can lead to putting unseasonable weight to the evidence being analyzed, therefore not properly representing the analysis and interpretation being reported. This calibration step is out of the scope of this dissertation but is currently in progress and part of an ongoing more extensive research project in our group. The study conducted here will serve as a basis to evaluate different calibration methods and will serve as a basis to expand evaluating the GSR evidence at activity level propositions. 


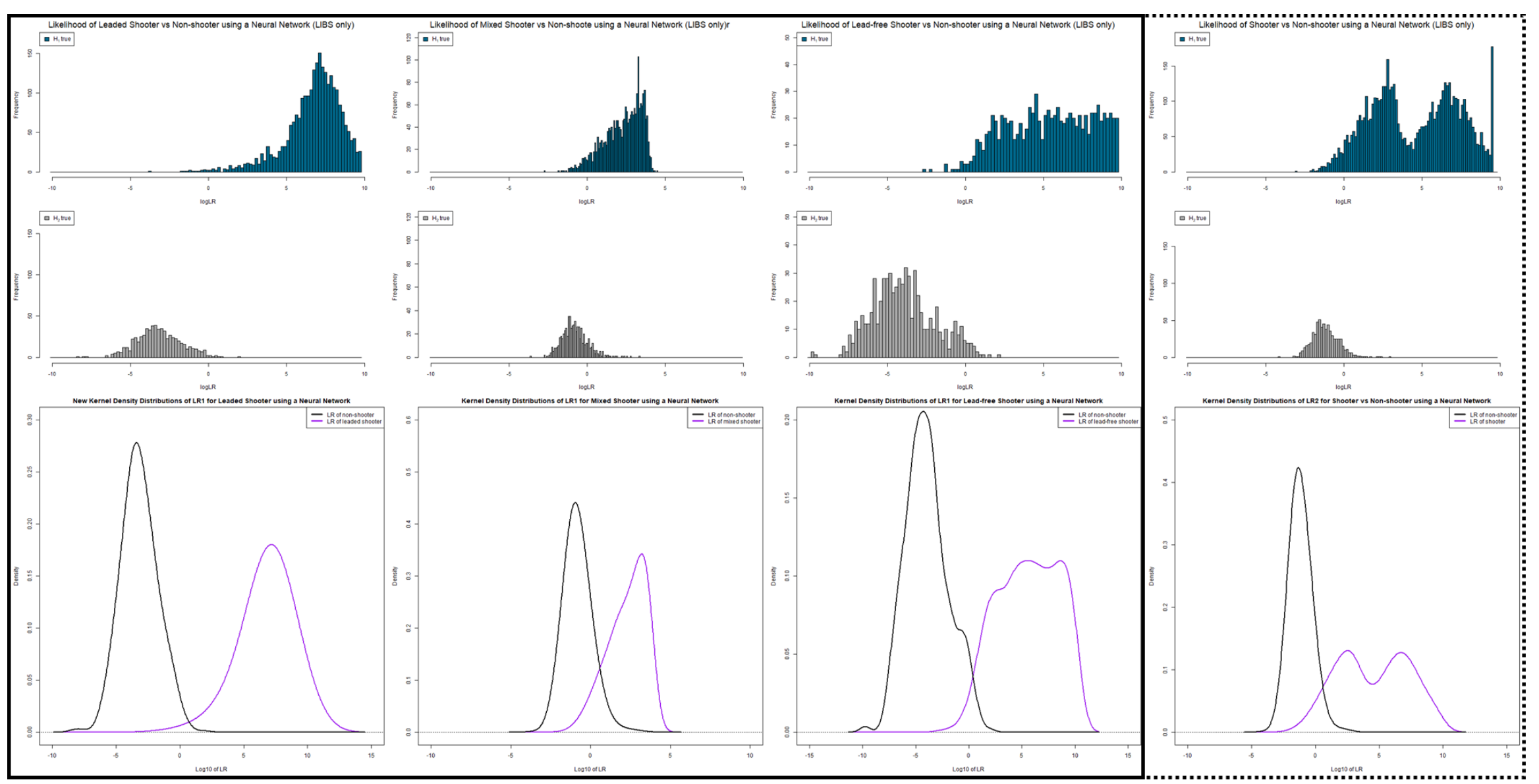

Figure 14: Histograms (top) with the corresponding KDF below. From left to right: leaded shooter and low risk populations, mixed shooter and low risk populations, lead free and low risk populations, and all shooter and low risk populations. 


\subsection{Conclusion}

The results from this large population have served to demonstrate LIBS's utility for the rapid classification of samples based on the presence or absence of GSR elemental profiles. The method showed it fits for purpose, detecting GSR from leaded and non-leaded ammunition fired with various firearms. Accuracy better than $90 \%$ was observed for datasets where more than eight variables were used for training the machine learning algorithm. An advantage of the machine learning methods is they provide probabilistic outputs that can be used to evaluate the weight or significance of the findings. An advantage afforded by LIBS is its speed of analysis, taking only over one minute to collect rich spectrochemical information from 25 different micro-areas. After analysis, we were also able to successfully confirm the samples by SEM-EDS since LIBS analysis consumes less than $0.5 \%$ of the sample. Elemental profiles observed by LIBS were successfully corroborated by SEM-EDS, demonstrating the utility of LIBS to evaluate elemental compositions of GSR particles in a quick and accurate approach.

In addition to the successful classification of the samples using machine learning techniques, the probabilistic outputs were exploited for determining the likelihood ratios for the collected data. Histograms and KDF demonstrated a reasonable separation between the two different ground truth hypotheses ant their respective populations of non-shooter and shooter samples (leaded, lead-free, and mixed). The likelihood ratio of $\mathrm{H}_{p}$, or $\mathrm{H}_{1}$, was consistently above zero and $\mathrm{H}_{d}$, or $\mathrm{H}_{2}$, was predominantly below zero, offering support to the respective propositions. Tippet plots showed low rates of misleading evidence and good discrimination power. Objective interpretation of GSR evidence can greatly assist the forensic community with future analysis and increased confidence of the results.

Use of a large population of samples arising from multiple types of individuals and ammunition has expanded our knowledge of not only modern, lead-free ammunition, but also the background levels of possible IGSR elements of interest. A greater understanding of the frequency of these elements on the hands of individuals who were not involved in a firing event improves our ability to differentiate between actual IGSR created from a firing event and IGSR-like elements commonly present in the background population, which will hopefully reduce the false detection of IGSR. In turn, this will improve the error rates of IGSR detection and increase public confidence when IGSR is actually detected. All these steps will improve the forensic community with modern alternatives for IGSR detection, interpretation, and assistance to the trier of fact. 


\subsection{References}

(1) Speaker, P. J. Project FORESIGHT Annual Report, 2018-2019; West Virginia University, 2020.

(2) Burch, A. M.; Durose, M. R.; Walsh, K. A.; Tiry, E.; Statistics, B. of J. Publicly Funded Forensic Crime Laboratories: Resources and Services, 2014; 2016.

(3) Standard Guide for Gunshot Residue Analysis by Scanning Electron Microscopy / Energy Dispersive X-Ray Spectrometry 1; West Conshohocken, PA, 2009. https://doi.org/10.1520/E1588-20.2.

(4) Ul-Hamid, A. A Beginners' Guide to Scanning Electron Microscopy. Springer: Cham, Switzerland 2018. https://doi.org/10.1007/978-3-319-98482-7.

(5) Ritchie, N. W. M.; DeGaetano, D.; Edwards, D.; Niewoehner, L.; Platek, F.; Wyatt, J. M. Proposed Practices for Validating the Performance of Instruments Used for Automated Inorganic Gunshot Residue Analysis. Forensic Chem. 2020, 20, 100252. https://doi.org/https://doi.org/10.1016/j.forc.2020.100252.

(6) Feeney, W.; Vander Pyl, C.; Bell, S.; Trejos, T. Trends in Composition, Collection, Persistence, and Analysis of IGSR and OGSR: A Review. Forensic Chem. 2020, 19. https://doi.org/10.1016/j.forc.2020.100250.

(7) Fambro, L. A.; Vandenbos, D. D.; Rosenberg, M. B.; Dockery, C. R. Laser-Induced Breakdown Spectroscopy for the Rapid Characterization of Lead-Free Gunshot Residues. Appl. Spectrosc. 2017, 71 (4), 699-708. https://doi.org/10.1177/0003702816689099.

(8) Rinke-Kneapler, C. N.; Sigman, M. E. Applications of Laser Spectroscopy in Forensic Science. In Laser Spectroscopy for Sensing: Fundamentals, Techniques and Applications; Baudelet, M., Ed.; Woodhead Publ Ltd: Cambridge, 2014; pp 461-495. https://doi.org/10.1533/9780857098733.3.461.

(9) Rosenberg, M. B.; Dockery, C. R. Determining the Lifetime of Detectable Amounts of Gunshot Residue on the Hands of a Shooter Using Laser-Induced Breakdown Spectroscopy. Appl. Spectrosc. 2008, 62 (11), 1238-1241. https://doi.org/10.1366/000370208786401473.

(10) Pyl, C. Vander; Ovide, O.; Ho, M.; Yuksel, B.; Trejos, T. Spectrochemical Mapping Using Laser Induced Breakdown Spectroscopy as a More Objective Approach to Shooting Distance Determination. Spectrochim. Acta Part B At. Spectrosc. 2019, 152, 93-101. 
https://doi.org/https://doi.org/10.1016/j.sab.2018.12.010.

(11) Dockery, C. R.; Goode, S. R. Laser-Induced Breakdown Spectroscopy for the Detection of Gunshot Residues on the Hands of a Shooter. Appl. Opt. 2003, 42 (30), 6153-6158. https://doi.org/10.1364/ao.42.006153.

(12) Silva, M. J.; Cortez, J.; Pasquini, C.; Honorato, R. S.; Paima, A. P. S.; Pimentel, M. F.; Paim, A. P. S.; Pimentel, M. F. Gunshot Residues: Screening Analysis by Laser-Induced Breakdown Spectroscopy. J. Braz. Chem. Soc. 2009, 20 (10), 1887-1894. https://doi.org/10.1590/S0103-50532009001000017.

(13) Vander Pyl, C.; Morris, K.; Arroyo, L.; Trejos, T. Assessing the Utility of LIBS in the Reconstruction of Firearm Related Incidents. Forensic Chem. 2020, 19 (May), 100251. https://doi.org/10.1016/j.forc.2020.100251.

(14) Trejos, T.; Vander Pyl, C.; Menking-Hoggatt, K.; Alvarado, A. L.; Arroyo, L. E. Fast Identification of Inorganic and Organic Gunshot Residues by LIBS and Electrochemical Methods . Forensic Chem. 2018, 8, 146-156. https://doi.org/doi.org/10.1016/j.forc.2018.02.006.

(15) Lopez-Lopez, M.; Alvarez-Llamas, C.; Pisonero, J.; Garcia-Ruiz, C.; Bordel, N. An Exploratory Study of the Potential of LIBS for Visualizing Gunshot Residue Patterns. Forensic Sci. Int. 2017, 273, 124-131. https://doi.org/10.1016/j.forsciint.2017.02.012.

(16) Menking-Hoggatt, K.; Arroyo, L.; Curran, J.; Trejos, T. Novel LIBS Method for MicroSpatial Chemical Analysis of Inorganic Gunshot Residue. J. Chemom. 2019, e3208, 13.

(17) Rammelkamp, K.; Schröder, S.; Ortenzi, G.; Pisello, A.; Stephan, K.; Baqué, M.; Hübers, H.-W.; Forni, O.; Sohl, F.; Thomsen, L.; Unnithan, V. Field Investigation of Volcanic Deposits on Vulcano, Italy Using a Handheld Laser-Induced Breakdown Spectroscopy Instrument. Spectrochim. Acta Part B At. Spectrosc. 2021, 177, 106067. https://doi.org/https://doi.org/10.1016/j.sab.2021.106067.

(18) Rakovský, J.; Čermák, P.; Musset, O.; Veis, P. A Review of the Development of Portable Laser Induced Breakdown Spectroscopy and Its Applications. At. Spectrosc. 2014, 101 (C), 269-287.

(19) Senesi, G. S.; Harmon, R. S.; Hark, R. R. Field-Portable and Handheld Laser-Induced Breakdown Spectroscopy: Historical Review, Current Status and Future Prospects. Spectrochim. Acta Part B At. Spectrosc. 2021, 175, 106013. 
https://doi.org/https://doi.org/10.1016/j.sab.2020.106013.

(20) Dona-Fernandez, A.; de Andres-Gimeno, I.; Santiago-Toribio, P.; Valtuille-Fernandez, E.; Aller-Sanchez, F.; Heras-Gonzalez, A. Real-Time Detection of GSR Particles from Crime Scene: A Comparative Study of SEM/EDX and Portable LIBS System. Forensic Sci. Int. 2018, 292, 167-175. https://doi.org/10.1016/j.forsciint.2018.09.021.

(21) Tucker, W.; Lucas, N.; Seyfang, K. E.; Kirkbride, K. P.; Popelka-Filcoff, R. S. Gunshot Residue and Brakepads: Compositional and Morphological Considerations for Forensic Casework. Forensic Sci. Int. 2017, 270, 76-82.

(22) Cardinetti, B.; Ciampini, C.; D’Onofrio, C.; Orlando, G.; Gravina, L.; Ferrari, F.; Di Tullio, D.; Torresi, L. X-Ray Mapping Technique: A Preliminary Study in Discriminating Gunshot Residue Particles from Aggregates of Environmental Occupational Origin. Forensic Sci. Int. 2004, 143 (1), 1-19. https://doi.org/10.1016/j.forsciint.2004.01.019.

(23) Seyfang, K. E.; Lucas, N.; Redman, K. E.; Popelka-Filcoff, R. S.; Kobus, H. J.; Kirkbride, K. P. Glass-Containing Gunshot Residues and Particles of Industrial and Occupational Origins: Considerations for Evaluating GSR Traces. Forensic Sci. Int. 2019, 298, 284 297. https://doi.org/https://doi.org/10.1016/j.forsciint.2019.03.010.

(24) Grima, M.; Butler, M.; Hanson, R.; Mohameden, A. Firework Displays as Sources of Particles Similar to Gunshot Residue. Sci. Justice 2012, 52 (1), 49-57. https://doi.org/10.1016/j.scijus.2011.04.005.

(25) Ali, L.; Brown, K.; Castellano, H.; Wetzel, S. J. A Study of the Presence of Gunshot Residue in Pittsburgh Police Stations Using SEM/EDS and LCMS/MS. J. Forensic Sci. 2016, 61 (4), 928-938. https://doi.org/10.1111/1556-4029.13077.

(26) Cook, M. Gunshot Residue Contamination of the Hands of Police Officers Following Start-of-Shift Handling of Their Firearm. Forensic Sci. Int. 2016, 269, 56-62. https://doi.org/10.1016/j.forsciint.2016.11.002.

(27) Charles, S.; Geusens, N. A Study of the Potential Risk of Gunshot Residue Transfer from Special Units of the Police to Arrested Suspects. Forensic Sci. Int. 2012, 216 (1), 78-81. https://doi.org/https://doi.org/10.1016/j.forsciint.2011.08.022.

(28) Berk, R. E.; Rochowicz, S. A.; Wong, M.; Kopina, M. A. Gunshot Residue in Chicago Police Vehicles and Facilities: An Empirical Study. J. Forensic Sci. 2007, 52 (4), 838841. https://doi.org/10.1111/j.1556-4029.2007.00457.x. 
(29) Anders, D. H.; Miller, S. A.; Graziano, C. R.; Castellano, J.; Conte, J. Technical Note: Presence of Gunshot Residue in and around a Police Station. Int. J. Legal Med. 2020, 134 (6), 2195-2198. https://doi.org/10.1007/s00414-020-02357-2.

(30) Lucas, N.; Cook, M.; Kirkbride, K. P.; Kobus, H. Gunshot Residue Background on Police Officers: Considerations for Secondary Transfer in GSR Evidence Evaluation. Forensic Sci. Int. 2019, 297 (2019), 293-301. https://doi.org/10.1016/j.forsciint.2019.02.017.

(31) Głomb, P.; Romaszewski, M.; Cholewa, M.; Domino, K. Application of Hyperspectral Imaging and Machine Learning Methods for the Detection of Gunshot Residue Patterns. Forensic Sci. Int. 2018, 290, 227-237.

https://doi.org/https://doi.org/10.1016/j.forsciint.2018.06.040.

(32) Mohri, M.; Rostamizadeh, A.; Talwalkar, A. Foundations of Machine Learning. MIT Press: Cambridge, MA 2012.

Maitre, M.; Kirkbride, K. P.; Horder, M.; Roux, C.; Beavis, A. Current Perspectives in the Interpretation of Gunshot Residues in Forensic Science: A Review. Forensic Sci. Int. 2017, 270, 1-11.

(34) Gallidabino, M.; Weyermann, C.; Romolo, F. S.; Taroni, F. Estimating the Time since Discharge of Spent Cartridges: A Logical Approach for Interpreting the Evidence. Sci. Justice 2013, 53 (1), 41-48. https://doi.org/10.1016/j.scijus.2011.12.004.

(35) Biedermann, A.; Bozza, S.; Taroni, F. Probabilistic Evidential Assessment of Gunshot Residue Particle Evidence (Part I): Likelihood Ratio Calculation and Case Pre-Assessment Using Bayesian Networks. Forensic Sci. Int. 2009, 191 (1-3), 24-35. https://doi.org/10.1016/j.forsciint.2009.06.004.

(36) Bell, S.; Seitzinger, L. From Binary Presumptive Assays to Probabilistic Assessments: Differentiation of Shooters From Non-Shooters Using IMS, OGSR, Neural Networks, and Likelihood Ratios. Forensic Sci. Int. 2016, 263, 176-185.

(37) Biedermann, A.; Bozza, S.; Taroni, F. Probabilistic Evidential Assessment of Gunshot Residue Particle Evidence (Part II): Bayesian Parameter Estimation for Experimental Count Data. Forensic Sci. Int. 2011, 206 (1-3), 103-110. https://doi.org/10.1016/j.forsciint.2010.07.009.

(38) Ott, C. E.; Dalzell, K. A.; Calderon-Arce, P. J.; Alvarado-Gamez, A. L.; Trejos, T.; Arroyo, L. E.; Calderón-Arce, P. J.; Alvarado-Gámez, A. L.; Trejos, T.; Arroyo, L. E. 
Evaluation of the Simultaneous Analysis of Organic and Inorganic Gunshot Residues Within a Large Population Data Set Using Electrochemical Sensors*(,)Dagger. J. Forensic Sci. 2020, 65 (6), 1935-1944. https://doi.org/10.1111/1556-4029.14548.

(39) Kleinbaum, D. G.; Klein, M. Logistic Regression : A Self-Learning Text. 3rd ed. Springer: New York 2010. https://doi.org/10.1007/978-1-4419-1742-3.

(40) Marini, F. Artificial Neural Networks in Foodstuff Analyses: Trends and Perspectives A Review. Anal. Chim. Acta 2009, 635 (2), 121-131. https://doi.org/10.1016/j.aca.2009.01.009.

(41) Wasserman, P. D. Neural Computing : Theory and Practice; Van Nostrand Reinhold: New York, 1989.

(42) Basu, S. Formation of Gunshot Residues. J. Forensic Sci. 1982, 27 (1), 72-91. https://doi.org/10.1520/jfs11453j.

(43) Gauriot, R.; Gunaratnam, L.; Moroni, R.; Reinikainen, T.; Corander, J. Statistical Challenges in the Quantification of Gunshot Residue Evidence. J. Forensic Sci. 2013, 58 (5), 1149-1155. https://doi.org/10.1111/1556-4029.12179.

(44) Damary, N. K.; MandelY, M.; Levin, N.; Izraeli, E. Calculation of Likelihood Ratios for Gunshot Residue Evidence-Statistical Aspects. Law, Probab. Risk 2016, 15 (2), 107-125. https://doi.org/10.1093/lpr/mgw001.

(45) Taroni, F.; Biedermann, A.; Bozza, S.; Garbolino, P. 1955-; Aitken, C. G. G. T. A.-T. T.-Bayesian Networks for Probabilistic Inference and Decision Analysis in Forensic Science. Second edi. Wiley: Chichester, West Sussex 2014. https://doi.org/10.1002/9781118914762 LK https://libwvu.on.worldcat.org/oclc/883246797.

(46) Zadora, G.; Martyna, A.; Ramos, D.; Aitken, C. T. A.-T. T.-. Statistical Analysis in Forensic Science : Evidential Value of Multivariate Physicochemical Data LK Https://Libwvu.on.Worldcat.Org/Oclc/858778356. NV-1 o. John Wiley \& Sons Inc.: Chichester, West Sussex 2014.

(47) Aitken, C. G. G.; Taroni, F. Statistics and the Evaluation of Evidence for Forensic Scientists LK - Https://Libwvu.on.Worldcat.Org/Oclc/54953145, 2nd ed.; Wiley: Chichester, England; SE - xxx, 509 pages : illustrations ; 24 cm., 2004.

(48) Curran, J. M.; Hicks, T. N.; Buckleton, J. S. Forensic Interpretation of Glass Evidence LK 
- Https://Libwvu.on.Worldcat.Org/Oclc/43936726; CRC Press: Boca Raton SE - 178 pages : illustrations ; $24 \mathrm{~cm}, 2000$.

(49) Woodman, P. A.; Spiranovic, C.; Julian, R.; Ballantyne, K. N.; Kelty, S. F. The Impact of Chemical Trace Evidence on Justice Outcomes: Exploring the Additive Value of Forensic Science Disciplines. Forensic Sci. Int. 2020, 307, 110121. https://doi.org/https://doi.org/10.1016/j.forsciint.2019.110121.

(50) Peterson, J. L.; Crim, D.; Hickman, M. J.; Strom, K. J.; Johnson, D. J. Effect of Forensic Evidence on Criminal Justice Case Processing. J. For. Sci. 2013, 58, S78-S90.

(51) Cook, R.; Evett, I. W.; Jackson, G.; Jones, P. J.; Lambert, J. A. A Hierarchy of Propositions: Deciding Which Level to Address in Casework. Sci. Justice 1998, 38 (4), 231-239. https://doi.org/10.1016/s1355-0306(98)72117-3.

(52) Vergeer, P.; Leegwater, A. J.; Slooten, K. Evaluation of Glass Evidence at Activity Level: A New Distribution for the Background Population. Forensic Sci. Int. 2020, 316, 110431. https://doi.org/https://doi.org/10.1016/j.forsciint.2020.110431.

(53) Jackson, G.; Jones, S.; Booth, G.; Champod, C.; IW, E. The Nature of Forensic Science Opinion--a Possible Framework to Guide Thinking and Practice in Investigations and in Court Proceedings. LK - Https://Libwvu.on.Worldcat.Org/Oclc/111036408. Sci. justice J. Forensic Sci. Soc. TA - TT - 2006, 46 (1), 33-44.

(54) Curran, J. M.; Hicks, T. N. Glass Evidence: Bayesian Approach To. Wiley Encycl. Forensic Sci. 2009, 1. https://doi.org/10.1002/9780470061589.fsa136.

(55) Bolck, A.; Stamouli, A. Likelihood Ratios for Categorical Evidence; Comparison of LR Models Applied to Gunshot Residue Data. Law Probab. Risk 2017, 16 (3), 19. Cardinetti, B.; Ciampini, C.; Abate, S.; Marchetti, C.; Ferrari, F.; Di Tullio, D.; D’Onofrio, C.; Orlando, G.; Gravina, L.; Torresi, L.; Saporita, G. A Proposal for Statistical Evaluation of the Detection of Gunshot Residues on a Suspect. Scanning 2006, 28 (3), 142-147. https://doi.org/10.1002/sca.4950280302.

(57) van Es, A.; Wiarda, W.; Hordijk, M.; Alberink, I.; Vergeer, P. Implementation and Assessment of a Likelihood Ratio Approach for the Evaluation of LA-ICP-MS Evidence in Forensic Glass Analysis. Sci. Justice 2017, 57 (3), 181-192. https://doi.org/10.1016/j.scijus.2017.03.002.

(58) Prusinowski, M.; Brooks, E.; Trejos, T. Development and Validation of a Systematic 
Approach for the Quantitative Assessment of the Quality of Duct Tape Physical Fits.

Forensic Sci. Int. TA - TT - 2020, 307, 110103.

https://doi.org/10.1016/j.forsciint.2019.110103 LK -

https://libwvu.on.worldcat.org/oclc/8465129067.

(59) Kara, I.; Sarikavak, Y.; Lisesivdin, S. B.; Kasap, M. Evaluation of Morphological and Chemical Differences of Gunshot Residues in Different Ammunitions Using SEM/EDS Technique. Environ. Forensics 2016, 17 (1), 68-79.

https://doi.org/10.1080/15275922.2015.1133729.

(60) Jackson, G. The Scientist and the Scales of Justice. Sci. Justice TA - TT - 2000, 40 (2), 81-85. https://doi.org/10.1016/S1355-0306(00)71947-2 LK https://libwvu.on.worldcat.org/oclc/4932963963.

(61) Corzo, R.; Hoffman, T.; Weis, P.; Franco-Pedroso, J.; Ramos, D.; Almirall, J. The Use of LA-ICP-MS Databases to Calculate Likelihood Ratios for the Forensic Analysis of Glass Evidence. Talanta TA - TT - 2018, 186, 655-661. https://doi.org/10.1016/j.talanta.2018.02.027 LK https://libwvu.on.worldcat.org/oclc/7986023288.

(62) Vergeer, P.; van Es, A.; de Jongh, A.; Alberink, I.; Stoel, R. Numerical Likelihood Ratios Outputted by LR Systems Are Often Based on Extrapolation: When to Stop Extrapolating? Sci. Justice 2016, 56 (6), 482-491. https://doi.org/https://doi.org/10.1016/j.scijus.2016.06.003. 


\section{Section 7}

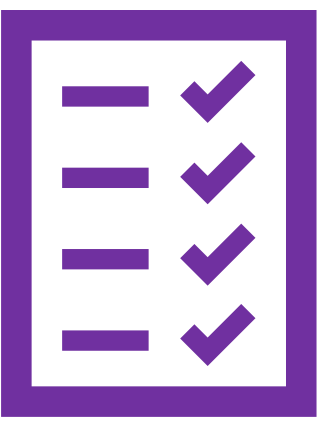

Summary

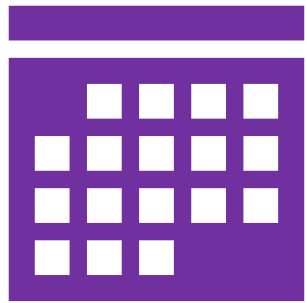

Future

work

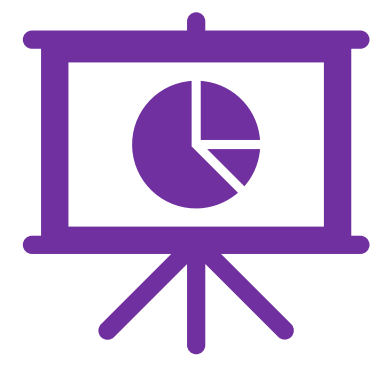

Impact

Overall Conclusions and Future Directions 


\section{Overall Conclusions and Future Directions}

\subsection{Summary of the Study}

This study met our overall goal of enhancing the reliability of GSR evidence through the development of rapid analytical tools that provided an increased body of knowledge on the chemical composition of modern ammunition residues and a novel interpretation framework for the quantitative assessment of the evidential value of GSR. We anticipate that the methodologies, standard materials, and the large population dataset will become accessible to forensic practitioners to complement and modernize current practice. The main findings and achievements of this study are discussed in the following sections.

\subsubsection{Objective 1: Qualitative and Quantitative Characterization of Multiple Types of Standard and Lead-Free Primers using a Novel Collection and Analytical Approach}

The goal of this objective was to qualitatively and quantitatively characterize different leaded and lead-free primers from modern ammunition using three analytical techniques and determine their viability as $p$ GSR standards for future development and validation of other methods for GSR detection. ICP-MS was utilized to qualitatively and quantitatively determine the bulk concentration of IGSR elements of interest. SEM-EDS was utilized to provide complementary elemental information and examine the morphology of the particles suspended in the $p \mathrm{GSR}$ standard. Both techniques were also used to monitor stability of the standard over time to determine the shelf life and stability of the suspended particles. Lastly, LIBS was utilized as another level of complementary elemental information, but also as a proof of application for use as a daily quality control sample to ensure proper instrument performance.

A full validation of the bulk ICP-MS digestion method was successfully completed, and the method was used to evaluate the stability of the bulk concentration of the $p$ GSR standards over time. Robustness testing was necessary to ensure the close monitoring of critical parameters for obtaining reliable and reproducible data. These factors were determined to be acid concentration, time between preparation and digestion, and time between digestion and analysis, all of which were closely monitored using control samples throughout the process. The validation showed the 
LOD and LOQ were in the low ppb range and the linear working range of the calibration curve was able to quantify these low concentrations accurately. Bias for most elements was less than $10 \%$, with recovery rates between $90-110 \%$, intraday variability better than $5 \%$, and interday variability around $15 \%$. With the results of the validation, the digestion method was considered fit for the purpose of monitoring up to 34 elements in low concentrations over time.

Once the validation was completed, the method was incorporated into the multi-analytical scheme for the characterization of the $p \mathrm{GSR}$ standards by ICP-MS, SEM-EDS, and LIBS. At the end of the study, 10 leaded and 5 lead-free primers were tested for a full year of stability (3 replicates each for 45 total stock suspensions), 5 additional lead-free primers were test for 24weeks ( 3 replicates each for 15 total suspensions), and 5 primers advertised as lead-free were only analyzed to 12 -weeks ( 3 replicates each for 15 total) due to the primer composition actually being leaded and containing lead, barium, and antimony. All the samples listed were tested by ICP-MS and demonstrated bulk concentration stability over the course of their stability study using ANOVA and post-HOC Tukey Kramer testing with an alpha value of 0.5. While some were not tested for a full year due to purchasing them later in the study or the primer composition not being truly lead-free, we can infer from the similarities in elemental composition that the $p$ GSR standards should also demonstrate the same stability as the standards studied for a full year.

Additionally, SEM-EDS was completed on replicate B for each of the different collected primer types and was tested at week 0,6, 12, and 52. Analysis of the pGSR standards complemented the elemental information provided by ICP-MS and also captured the morphological information that other methods of characterization could not provide. SEM-EDS confirmed the presence of the same elements and also provided the possible elemental combinations that could be found in a single particle. We also saw that elements that were above the LOD, but not the LOQ by ICP-MS, tended to be the elements that were not consistently found in every particle, while the elements above the LOQ were almost always detected in the particles. As for morphology, both spheroid and irregular particles were detected, and when compared to authentic samples later, this pattern was also observed. Therefore, the $p \mathrm{GSR}$ created proved to mimic authentic IGSR.

Lastly, LIBS was successfully applied as a proof of application. All the collected samples and replicates were analyzed by LIBS to provide another set of complementary elemental information for the standards. All the elements above the LOD for LIBS were observed, further 
strengthening our confidence in the concentrations determined by ICP-MS. As the proof of application, the samples were used as reference materials for the development of the micro-spatial rapid LIBS method for IGSR detection and daily quality control. Prior to routine analysis, a few $p$ GSR samples were tested to ensure the instrument's performance. Standards such as the one developed here provided an invaluable addition to the reliability of our developed method.

The success of this first objective provided critical groundwork for the second objective. Information about the elemental composition of the different leaded and lead-free primers provided "ground truth" knowledge for the next step of the project. While all the leaded primers had lead, barium, and antimony, we determined that one only contained lead and antimony. Also, the elemental composition of lead-free primers was very diverse, some had only light elements such as copper and potassium, other contain titanium and zinc, while others only had barium or bismuth. Without this knowledge, the next step of the project would have been difficult since the elemental composition was necessary to detect IGSR and many of the elemental combinations determined here were not included in the standard criteria for IGSR detection. In addition to laying the groundwork for the population study, this study also presents the forensic community with new information of IGSR elements of interest to include in the future in their interpretation of GSR evidence.

\subsubsection{Objective 2: Validation of the Reliability of LIBS and SEM-EDS by the Statistical Analysis of a Large Population}

The goal of this objective was to validate the reliability of LIBS and SEM-EDS through the statistical analysis of a large population set that included hand residues from known shooters and non-shooters using the characterized leaded and lead-free ammunitions, along with low and high-risk background populations. At the end of this study, 350 low-risk background sets, 105 high-risk background sets, 200 leaded shooter sets, 100 lead-free shooter sets, and 220 mixed shooter sets were analyzed. This collection resulted in a total of over 2,900 stubs analyzed by LIBS and currently over 150 confirmed by SEM-EDS, with an additional 50 high-risk stubs in progress. From this we would be able to provide the forensic science community valuable information about IGSR. Study such as this lay the groundwork for interpretation of evidence at the source level and future studies to incorporate the activity aspects of transfer, persistence and background occurrence. 
Prior to completing the large population study, we developed and validated a micro-spatial LIBS method for the rapid detection of IGSR using an additional 51 low-risk background sets (102 stubs) and 56 leaded shooter sets (224 stubs). A critical threshold method and three types of machine learning were applied to classify the data and served as the basis for justification of the classification methods used in the larger population study. Accuracies of the classification methods for this preliminary pilot set ranged from 87-100\%, showing promising performance. Other benefits of the method were the addition of spatial information in the form of 100 um diameter spots, the increase in spectral information collected (1 spectrum for the line method and 25 spectra with spatial information for the micro-spatial method), and even less consuming of the stub since each spot only required 2 laser shots and barely damaged the carbon substrate. The use of two laser shots also improved the signal to noise because the analysis was more superficial and not as much of the carbon adhesive background contributed to the noise.

Lastly, we completed the large population study using the micro-spatial LIBS method, we obtain accuracies over $90 \%$, depending on the classification method applied. We were also able to perform SEM-EDS confirmation on the same sample analyzed beforehand by LIBS, on approximately 200 stubs from the various populations. Since SEM-EDS is a time-consuming technique of analysis, a batch of four stubs took at least a day to complete and showed to be the bottleneck of this project. The LIBS method was able to analyze hundreds of stubs per day and quickly turn around the results (same day), therefore demonstrating the rapid response of the LIBS and the superior efficiency it could provide to firearm investigations.

Data gathered from the population study was also analyzed by logistic regression and a neural network to provide probabilistic interpretation of the data. The two machine learning methods were trained and provided probabilities for the data classification within seconds. The probabilities were used to calculate the likelihood ratios for the different populations. From the calculated likelihood ratios, histograms, Tippet plots, and Kernel Density Functions were plotted to evaluate the data. These graphs and plots provided empirical measurements of the performance of likelihood ratio and assisted in visualizing the separation between the different population groups and the discrimination of the method. There was a clear separation between the responses observed for low-risk, non-shooter background population ( $\log 10$ likelihood ratio <0) and the different known-shooter population ( $\log 10$ likelihood ratio $>0$ ). This study showed the ability to use LIBS data to perform further Bayesian interpretation of GSR. 
All the research and data collected for this large population study lays the groundwork for future studies about GSR. We have been able to provide performance and error rates for our different classification methods. Preliminary statistical analysis using machine learning demonstrates the power of implementing objective algorithms into decision making and the ability to calculate likelihood ratios for Bayesian interpretation. A study this large provides future researchers with valuable insight into the detection of GSR in a forensic setting.

\subsection{Future Work}

The study completed has only begun to touch the surface of research needed about GSR. The first objective of the study tested the elemental concentrations of different types of leaded and lead-free primers. Out of the ten lead-free primers studied, we saw a variety of elements present, some of them not yet included in the current standards or reported in the literature. As with any manufactured item, formulations change over time and as trace evidence analysts, we need to keep updated on how the market and formulations are changing. Also, the stability of the already collected tailor-made suspensions will continue to be monitored to determine the shelf-life.

The $p$ GSR standards were successfully used in our laboratory, and the next stage includes an interlaboratory study to demonstrate the reliability and reproducibility of the collected standards on other instruments and analysts. Thankfully, the project has gained the attention of crime laboratories and academic institutions all over the country, who have reached out to participate in such collaborative exercise.

While this study collected a large number of samples, there is still much research to be completed in order to strengthen GSR analysis. Analysis of the high-risk samples is currently in progress and will be included in the statistical interpretation in the near future. Bigger picture though, this study has only focused on hand samples but would benefit for the testing of different items, such as clothing and surfaces. Additionally, samples such as these are not compatible with SEM-EDS due to chamber size requirements and time restrictions.

Also, the study could continue to grow by adding more hand samples from different populations and ammunition over time. With the addition of more samples, we can increase our confidence in the ability of the method to differentiate between the groups. We can also include different background populations to determine if different locations effect the critical threshold for 
any possible IGSR elements. This would provide location specific background information to GSR analysts about the elements common present in their environment.

The most challenging and beneficial next stage is the implementation of the probabilistic interpretation. High-risk background samples need to be included to determine where they fall in the distributions. Also, the likelihood ratios calculated need to be calibrated to ensure they offer realistic numbers and returning values that would prevent misleading perceptions of the weight of the evidence. We anticipate using this base knowledge, to apply Bayesian networks in the future, which can take into account deposition, transfer, persistence, activity, and the analytical process. Bayesian Networks can provide the forensic community and the triers of fact with a visual of how all the different parts of trace evidence interact and their effect on the final outcome. Overall, the study completed here will help future researchers understand the gaps in GSR analysis and the future work that needs to be completed.

\subsection{Impact on the Criminal Justice System}

Over the course of this project results have been disseminated and presented to the forensic and scientific community and received very positive feedback. This had led to multiple collaborators reaching out to our research group with interest in interlaboratory studies and pilot programs for testing the method. The development of the rapid LIBS method could fulfill a great need for faster GSR evidence processing and therefore provide law enforcement officers with investigative leads sooner.

In order to develop new methods, a GSR standard was validated and optimized to serve as quality control tests. Currently, only a synthetic GSR SEM-EDS standard is available on the market, but the sample is not consumable. Our study on the tailor-made $p$ GSR standard provided a foundation for the development of a IGSR standard that can be used for testing diverse analytical methods, including the traditional SEM-EDS analysis. Moreover, the study of 25 primers types (3 replicates resulting in a total of 75 standard suspensions) for objective increased knowledge about the elemental composition of GSR originating from modern primers.

Another impact of the project are the results of the population study and probabilistic interpretation. During this project, over 2,900 GSR stubs were analyzed by LIBS. This large sample set would have taken several years to be analyzed by SEM-EDS. Therefore, for the first time, we can complete a population study large enough to begin drawing meaningful statistical 
conclusions. A study of this kind could help to enhance the interpretation of evidence and strengthen the analyst conclusions when writing reports and presenting evidence in court. 
Korina Menking-Hoggatt

$304-444-3827$

Morgantown, $W V$

klmenkinghoggatt@mix.wvu.edu

\section{Education}

\section{West Virginia University}

Doctorate of Philosophy in Forensic Science

$$
\text { Morgantown, } W V
$$

2018-present

Current GPA- 3.72

- Anticipated Dissertation defense May 2021, expected graduation Summer 2021

- Funded through graduate assistantship by the FIS Department from Jan 2018- Dec 2018

- Funded through NIJ STEM Graduate Fellowship Award Jan 2019-June 2021

West Virginia University

Morgantown, $W V$

2014-2017

\section{Master's of Science in Forensic Science}

GPA- 3.67

- Thesis topic on the economic benefits of portable instrumentation

- Graduated

- Funded through graduate teaching assistantship from the FIS Department

West Virginia State University $\quad$ Charleston, WV 2004-2009
Bachelor's of Science in Chemistry
Graduated Magna Cum Laude
GPA- 3.59

- Four-year recipient of the Promise Scholarship

- Member of National Honor Society

\section{PhD Dissertation Topic}

Characterization of Modern Ammunitions and Background Profiles: A Novel Approach and Probabilistic Interpretation of Inorganic Gunshot Residue.

\section{MS Thesis Topic}

The Economic Benefits of Portable Instrumentation on the Criminal Justice System:

A Comprehensive Return-on-Investment Analysis. 


\section{Work Experience}

\section{West Virginia University- Graduate Fellowship Research Assistant}

$$
\text { 304-293-2453 Morgantown, } W V
$$

1/2018-present

Graduate Research Assistant for the NIJ fellowship 2018-R2-CX-0009

Primary Investigator: Dr. Tatiana Trejos

- Organizes experimental design and collection of samples

- Performs instrumental and data analysis of collected samples

- Disseminates results in the form of peer-reviewed articles, oral presentations, and posters

- Creates training documents and standard operating procedures for developed methods

- Trains undergraduate and graduate students on developed methods

- Participates in collaborations with outside agencies, universities, and industrial partners

- Tracks and replaces inventory while monitoring the budget for the project

- Performs maintenance and necessary troubleshooting of instruments in the laboratory

- Effectively communicates with group and department through online and in-person platforms

- Regularly provides updates of research progress and challenges

\section{West Virginia University- Crime Scene Training Complex/ Teaching Assistant} 304-293-2453 Morgantown, $W V$

Graduate Assistant for the Crime Scene Training Complex

Supervisor: Casper Venter

- Primary teaching assistant for Crime Scene 1, Crime Scene 2, Firearms, and Bloodstain

- Secondary teaching assistant for Trace Evidence and Informatics

- Assisted in the daily operation of the WVU crime scene training complex

- Managed inventory and organization of training materials and courses

- Provided support to teachers, classes, and groups that use the training complex

- Designed and stages mock crime scenes and lab activities for the students

- Assisted in hands on training and lesson design for students

\section{Ruby Hospital- Clinical Laboratory}

304-598-4000 Morgantown, $W V \quad 1 / 2013-12 / 2015$

Microbiology Lab Technician

Supervisor: Amanda Murray ext. 4235

- Ordered, received, and processed microbiology specimens

- Held licensed to result moderately complicated microbiology tests in the laboratory

- Maintained instruments and performed quality control

- Answered questions and tracked specimens for other departments 


\section{Laboratory Corporation of America- Cytology Branch}

304-348-2100

Charleston, $W V$

$3 / 2007-8 / 2012$

Specialty Lab Assistant

Supervisor: Rita Affolter ext-2143

- Prepared and processed specimens on testing instruments

- Performed maintenance and troubleshooting on testing instruments

- Tracked specimens and results using multiple computer systems

- Assisted in the quality control and assurance process

- Trained in handling, shipping, and disposing of hazardous materials

\section{Special Professional Skills}

- Expertise in the fundamentals, operation, and maintenance of highly specialized analytical instrumentation such as Scanning Electron Microscopy-Energy Dispersive Electron Spectroscopy (SEM-EDS), Inductively Coupled Plasma-Mass Spectroscopy (ICP-MS), Laser AblationInductively Coupled Plasma-Mass Spectroscopy (LA-ICP-MS), and Laser Induced Breakdown Spectroscopy (LIBS)

- Proficient in the fundamentals and operation of analytical tools such as microscopy, FourierTransform Infrared Spectroscopy (FTIR), Raman Spectroscopy, Glass Refractive Index Measurement (GRIM) instrument, Gas Chromatography-Mass Spectrometry (GC-MS), Matrix Assisted Laser Desorption Ionization-Time of Flight Mass Spectrometry (MALDI-TOF MS), Nuclear magnetic resonance (NMR), DNA analysis (extraction, quantitation, amplification by Polymerase Chain Reaction (PCR), Capillary Electrophoresis (CE), and interpretation of allele calls), Thin-layer chromatography (TLC), numerous color tests used for drug screening (Cobalt Thiocyanate, Marquis test, Duquenois-Levine, etc), biological samples screening (Phenolphthalein, Luminol, Acid Phosphatase, etc), and gunshot residue distance determination (Sodium Rhodizonate and Griess test), Alternative Light Sources (ALS), and numerous immunoassay tests (Rapid Stain Identification, or RSID, and screening tests used by clinical laboratories)

- Experience in mentoring junior research students under several programs such as undergraduate research in the chemistry (CHEM 497) and forensic department (FIS 497), Summer Undergraduate Research Experience (SURE), undergraduate Research Apprenticeship Program (RAP), the forensic internship program (FIS 386), and masters graduate researchers (FIS 697)

- Experience in research design and validation of analytical methods

- Experience co-leading an externally funded project

- Excellent writing and communication skills as demonstrated by the submission of fellowship proposal that was awarded, and numerous publications and oral presentations at scientific venues listed in the sections below

- Experience as independent researcher in the areas of analytical and forensic chemistry, with a focus on elemental analysis techniques, chemometrics, machine learning algorithms, and development and management of large datasets

- Advanced photography skill for crime scene documentation and high-quality evidence for both documentation and comparison purposes 
- Experience with writing and updating Material Safety Data Sheets (MSDS) and Standard Operating Procedures (SOPs), and validation plans for laboratories

- Experience with understanding and interpreting the efficiency of laboratories based on metrics collected by LIMS systems, along with a basic understanding of laboratory economics and their application in publicly funded crime laboratories (Project FORESIGHT at WVU)

- Computational skills include Microsoft Office Suite, R programming language (RStudio), JMP Pro 15 by Statistical Analysis System (SAS), CrimePad (an application for crime scene management), ImageJ, Automated Fingerprint Identification System (AFIS), and numerous Laboratory Information Management System (LIMS)

\section{Professional Development}

\section{Publications}

1. Menking-Hoggatt, K.; Martinez, C.; Vander Pyl, C.; Heller, E.; Pollock, E. "Chip"; Arroyo, L.; Trejos, T. Development of Tailor-Made Inorganic Gunshot Residue (IGSR) Microparticle Standards and Characterization with a Multi-Technique Approach. Talanta 2021. https://doi.org/10.1016/j.talanta.2020.121984.

2. Menking-Hoggatt K.; Arroyo L.; Curran J.; Trejos T. Novel LIBS Method for Microspatial Chemical Analysis of Inorganic Gunshot Residues. Journal of Chemometrics 2019. https://doi.org/10.1002/cem.3208.

3. Trejos, T.; Vander Pyl, C.; Korina Menking-Hoggatt, K.; Alvarado, L.; Arroyo, L., Fast Identification of Inorganic and Organic Gunshot Residues by LIBS and Electrochemical Methods. Forensic Chemistry 2018. doi.org/10.1016/j.forc.2018.02.006.

\section{Presentations at Scientific Conferences}

1. March 11 ${ }^{\text {th }}, \mathbf{2 0 2 1}$. PITTCON 2021 virtual conference during the NIJ (National Institute of Justice) -Emerging Analytical Methods for Chemical and Biological Forensic Evidence Session. Tatiana Trejos, Luis Arroyo, Korina Menking-Hoggatt and Courtney Vander Pyl. LIBS as an emerging method for the detection of firearm discharge residues. PITTCON 2021 (co-author of an invited speaker)

2. March $9^{\text {th }}, \mathbf{2 0 2 1}$. PITTCON 2021 virtual conference. Luis Arroyo, Korina Menking Hoggatt, Colby Ott, Courntey Vander Pyl, Kourtney Dalzell, Bill Feeney. Detection of gunshot residues from leaded and non-leaded ammunition by electrochemical sensors and LIBS. PITTCON 2021 (co-author of an invited speaker)

3. February $\mathbf{1 8}^{\text {th }}, \mathbf{2 0 2 1}$. American Association of Forensic Science criminalistics virtual poster session. Kourtney A. Dalzell, Colby E. Ott, Korina Menking-Hoggatt, Tatiana Trejos, and Luis E. Arroyo. Detection of Lead-Free Inorganic and Organic Gunshot Residue Using LIBS, Electrochemistry, and Machine Learning. AAFS virtual meeting. (poster co-author)

4. February $\mathbf{1 8}^{\text {th }}, \mathbf{2 0 2 1}$. American Association of Forensic Science criminalistics virtual poster session. Courtney Vander Pyl, Korina Menking-Hoggatt, Claudia Martinez, and Tatiana Trejos. Application of Laser-Based Methods for the Analysis of Gunshot Residue Originating from Modern Ammunition. AAFS virtual meeting. (poster co-author)

5. January $\mathbf{2 5}^{\text {th }}, \mathbf{2 0 2 1}$. Crossing Forensic Borders: Event \#4. Korina Menking-Hoggatt, Colby Ott, Kourtney A. Dalzell, Courtney Vander Pyl, Luis E. Arroyo-Mora, and Tatiana 
Trejos. Novel Rapid Detection of Inorganic and Organic Gunshot Residues using LIBS and Electrochemistry: A Population Study. Online webinar. (co-presenter oral online presentation)

6. November $11^{\text {th }}, \mathbf{2 0 2 0} .7^{\circ}$ National Meeting of Forensic Chemistry $/ 4^{\circ}$ Meeting of the Brazilian Society of Forensic Sciences (ENQFor/SBCF) online Joint Congress. Korina Menking-Hoggatt and Luis Arroyo. Feasibility Study of Rapid Emerging Methods for the Analysis of Inorganic and Organic Gunshot Residues. (oral online presentation)

7. October 2020. FACSS SCIX 2020. Tatiana Trejos, Luis Arroyo, Colby Ott, Courtney Vander Pyl, Korina Menking-Hoggatt and Kourtney Dalzell. Investigative leads using LIBS and orthogonal methods in crime laboratories and in the field. Virtual conference and on demand oral abstracts. (co-author of oral presentation)

8. September 2020. Mid-Atlantic Association of Forensic Scientists conference 2020 Scholarship winner. Korina Menking-Hoggatt, Edward "Chip" Pollock, Emily Heller, Courtney Vander Pyl, Claudia Martinez, Tatiana Trejos. Inorganic Gunshot Residue (IGSR) Micro-particle Standard with Application to Method Development and Understanding Modern Ammunition. Online recording of the presentation due to COVID cancelation. (oral online presentation)

9. July 31 ${ }^{\text {st }}$ 2020. Current Trends in Forensic Trace Analysis online forensic symposium. Korina Menking-Hoggatt, Luis Arroyo, Colby Ott, and Tatiana Trejos. Characterizing Inorganic and Organic Gunshot Residue by Laser Induced Breakdown Spectroscopy and Electrochemistry. Online symposium. (poster online presentation)

10. July 31 ${ }^{\text {st }}, \mathbf{2 0 2 0}$. Current Trends in Forensic Trace Analysis online forensic symposium. Korina Menking-Hoggatt, Luis Arroyo, Colby Ott, and Tatiana Trejos. Characterizing Inorganic and Organic Gunshot Residue by Laser Induced Breakdown Spectroscopy and Electrochemistry. Current Trends in Forensic Trace Analysis online forensic symposium. Online symposium. (poster online presentation)

11. May $1^{\text {st }}, \mathbf{2 0 2 0}$. Online Forensic Graduate Symposium hosted by the Department of Forensic and Investigative Science at West Virginia University. Korina MenkingHoggatt, Claudia Martinez, Edward "Chip" Pollock, Emily Heller, Courtney Vander Pyl, Tatiana Trejos. Characterization of Modern Inorganic Gunshot Residue Micro-Particles for Enhancement of Forensic Analysis. ${ }^{\text {st }}$ WVU online symposium. (oral online presentation and symposium organizer)

12. May 31 ${ }^{\text {st }}, \mathbf{2 0 2 0}$. Online Forensic Graduate Symposium hosted by the Department of Forensic and Investigative Science at West Virginia University. Courtney Vander Pyl, Korina Menking-Hoggatt, Claudia Martinez, Tatiana Trejos. Fast Spectrochemical Methods and Micro-Particle Standards to Facilitate Transfer and Persistence Studies of Inorganic and Organic Gunshot Residues. Received Award for "Best Research e-Poster Presentation-First Place. $1^{\text {st }} \mathrm{WVU}$ online symposium. (co-author on poster)

13. April 2020. WVU Undergraduate Research Online Symposium. Emily Heller, Korina Menking-Hoggatt, Claudia Martinez-Lopez, and Tatiana Trejos. Analysis of Inorganic GSR Microparticles using Laser Induced Breakdown Spectroscopy (LIBS). (poster online presentation)

14. March $3^{\text {rd }}$, 2020. PITTCON conference. Korina Menking-Hoggatt, Edward Pollock, Emily Heller, Courtney Vander Pyl, Claudia Martinez, and Tatiana Trejos. Development and Characterization of Inorganic Gunshot Residue Micro-particles to Enhance Understanding of Modern Ammunition. Chicago, IL. (poster presentation) 
15. February $\mathbf{2 0}^{\text {th }}, \mathbf{2 0 2 0}$. American Association of Forensic Science. Korina MenkingHoggatt, James Curran, Courtney Vander Pyl, Colby Ott, Luis Arroyo, Tatiana Trejos. The Power of Statistics and Machine Learning Applied to Orthogonal Rapid Methods for the Identification of Inorganic Gunshot Residue (IGSR) and Organic Gunshot Residue (OGSR) Markers. Anaheim, CA. (oral presentation)

16. February $\mathbf{2 0}^{\mathbf{h}}, \mathbf{2 0 2 0}$. American Association of Forensic Science. Korina MenkingHoggatt, Edward Pollock, and Tatiana Trejos. A Novel Approach for the Collection and Characterization of Inorganic Gunshot Residue (IGSR) Standards. Anaheim, CA. (poster presentation)

17. February $\mathbf{1 9}^{\text {th }}, \mathbf{2 0 2 0}$. American Association of Forensic Science. Colby Ott, Pedro Calderon-Arce, Korina Menking-Hoggatt, Courtney Vander Pyl, Ana Alvarado-Gamez, Tatiana Trejos, Luis Arroyo. Edward Pollock, Tatiana Trejos. An Analysis of Organic and Inorganic Gunshot Residues (OGSR and IGSR) Via Electrochemical Methods with Screen-Printed Carbon Electrodes and Nanoparticle Modifications. Anaheim, CA. (poster presentation)

18. October $\mathbf{1 7}^{\text {th }}$, 2019. SciX Conference. Korina Menking-Hoggatt, Luis Arroyo, Colby Ott, and Tatiana Trejos. Characterizing Inorganic and Organic Gunshot Residue by Laser Induced Breakdown Spectroscopy and Electrochemistry. Palms Springs, CA. (poster presentation)

19. October $\mathbf{1 6}^{\text {th }}$, 2019. SciX Conference. Tatiana Trejos, Luis Arroyo, Emily Haase, Courtney Vander Pyl, Korina Menking-Hoggatt. Using LIBS for Elemental Signature Discovery in Forensic Applications. Palm Springs, CA (co-author of invited speaker)

20. May $9^{\text {th }}, 2019$. Mid-Atlantic Association of Forensic Science. Korina Menking-Hoggatt, Tatiana Trejos, and Luis Arroyo. Modern Fast Screening of Inorganic and Organic Gunshot Residue (GSR) by Laser-Induced Breakdown Spectroscopy and Electrochemistry. Morgantown, WV. (oral presentation)

21. February 21 ${ }^{\text {st }}$, 2019. American Association of Forensic Science. Korina Menking-Hoggatt and Tatiana Trejos. Laser-Induced Breakdown Spectroscopy as a Rapid Detection Technique for Gunshot Residue. Baltimore, MD. (poster presentation)

22. February $\mathbf{2 0}^{\text {th }}, 2019$. American Association of Forensic Science. Luis Arroyo, Korina Menking-Hoggatt and Tatiana Trejos. The Fusion of Electrochemical and Spectrochemical Data for the Detection of Organic and Inorganic Gunshot Residues (GSR). Baltimore, MD. (poster coauthor)

23. October 2018. SCIX annual meeting. Tatiana Trejos, Korina Menking-Hoggatt, Luis Arroyo. Chemical analysis and statistical interpretation of gunshot residues using LIBS and electrochemical sensors. Atlanta, GA. (co-author of invited speaker)

24. September 2018. Collaboration and technical scientific visit to the Sacramento County District Attorney Crime Lab. Korina Menking-Hoggatt, Luis Arroyo and Tatiana Trejos. Identification of Organic and Inorganic Gunshot Residues by Electrochemical and Spectrochemical Methods. Sacramento, CA. (five-day technical visit)

25. September 2018. Collaboration and technical scientific visit to the Sacramento County District Attorney Crime Lab. Korina Menking-Hoggatt and Tatiana Trejos. Characterization of Modern Ammunition and Background Profiles: A Novel Approach and Probabilistic Interpretation of Inorganic Gunshot Residue. Sacramento, CA. (five-day technical visit)

26. September 2018. Collaboration and technical scientific visit to the Sacramento County District Attorney Crime Lab. Korina Menking-Hoggatt, Courtney Vander Pyl and Tatiana Trejos. LIBS Applications for Firearm Distance Determinations. Sacramento, CA. (five-day technical visit) 
27. September 2018. Collaboration and technical scientific visit to the Sacramento County District Attorney Crime Lab. Korina Menking-Hoggatt and Tatiana Trejos. Return on Investment for Rapid LIBS Analysis. Sacramento, CA. (five-day technical visit)

28. July 2018. Annual Undergraduate Research Symposium. Oriana Ovide, Courtney Vander Pyl, Bayram Yuksel, Korina Menking-Hoggatt and Tatiana Trejos. Distance Determination Using Firearm Discharge Residues: Challenges of Color Tests and Benefits of LIBS. Morgantown, WV. (cop-author for the undergraduate SURE program poster presentation)

29. April $7^{\text {th }}, \mathbf{2 0 1 8}$. Attendee and $1^{\text {st }}$ place poster at the Chesapeake Bay DivisionInternational Association of Identification. Korina Menking-Hoggatt, Courtney Vander Pyl, Tatiana Trejos. Versatility of Laser Induced Breakdown Spectroscopy for Fast Detection of Gunshot Residues. Morgantown, WV. (poster presentation)

30. September 21 ${ }^{\text {st }}$, 2017. Mid-Atlantic Association of Forensic Scientists. Korina MenkingHoggatt, Luis Arroyo, and Tatiana Trejos. Fast Identification of Inorganic and Organic Gunshot Residues by LIBS and Electrochemical Methods. Cincinnati, OH. (poster presentation)

31. September 21 ${ }^{\text {st }}$, 2017. Combined MAFS, SAFS, ASTEE meeting. Tatiana Trejos, Korina Menking-Hoggatt, Luis Arroyo. Analysis of Gunshot Residues by laser-based spectrochemical methods and electrochemical sensors. Cincinnati, $\mathrm{OH}$. (co-author of an invited oral presentation)

32. April 22 ${ }^{\text {nd }}$ 2017. Chesapeake Bay Division- International Association of Identification. Korina Menking-Hoggatt and Bob O'Brien. The Benefits and Challenges of Crime Pad in the Field. Williamsburg, VA. (poster presentation)

\section{Awards and Funding Opportunities}

1. Recipient of Mid-Atlantic Association of Forensic Scientists conference 2020 Scholarship winner in September 2020.

2. Recipient of NIJ travel grant to attend and present at PITTCON May $1-4^{\text {th }}, 2020$ in the NIJ poster session.

3. Recipient of National Institute of Justice fellowship for STEM in Forensic Science; award number 2018-R2-CX-0009.

4. Recipient of the 2018 Blaney Fellowship. The award was used as travel funding to start a collaboration with the Sacramento County District Attorneys' Crim Laboratory (SCDACL) during the week of September 17 $7^{\text {th }}-20^{\text {th }}, 2018$.

5. $1^{\text {st }}$ place poster winner at the Chesapeake Bay Division- International Association of Identification on April 17 $7^{\text {th }}, 2018$ for poster titled Versatility of Laser Induced Breakdown Spectroscopy for Fast Detection of Gunshot Residues.

\section{Professional Development}

1. Attended and presented at American Academy of Forensic Science (AAFS) on February $15^{\text {th }}-19^{\text {th }}, 2021$, in virtual meeting format.

2. Attended and presented at the Crossing Forensic Borders: Event \#4 on January 25th, 2021, online.

3. Attended ASCLD-FRC Lightning Talk- Strategies or Organic and Inorganic GSR Analysis on December $3^{\text {rd }}, 2020$, online.

4. Attended the Forensics@ NIST virtual symposium on November $5^{\text {th }}-6^{\text {th }}, 2020$, online. 
5. Attended the 2020 Online Forensic Symposium for Current Trends in Forensic Trace Analysis five-day symposium on July $27^{\text {th }}-31^{\text {st }}, 2020$. Part of my dissertation research was presented by Dr. Luis Arroyo in a presentation titled Development of a Versatile IGSR Microparticle Standard.

6. Attended an Applied Spectra webinar titled Laser Ablation (LA-ICP-MS and LIBS) Application in Geology and Geochemistry online on May $8^{\text {th }}, 2020$.

7. Attended and presented at PITTCON conference on March $2^{\text {nd }}-5^{\text {th }}, 2020$, in Chicago, IL.

8. Visited Applied Spectra and SCDACL on February $24^{\text {th }}-27^{\text {th }}-, 2020$, to collaborate on the implementation of the rapid LIBS method developed by the Dr. Trejos Research Group at WVU in Sacramento, CA.

9. Attended and presented at American Academy of Forensic Science (AAFS) on February $17^{\text {th }}-21^{\text {st }}, 2020$, in Anaheim, CA.

10. Attended and presented at SCIX conference on October $15^{\text {th }}-18^{\text {th }}$, 2019, in Palm Springs, CA.

11. Attended an Agilent ICP-OES and MS webinar titled Removing Interferences with Automated Background Corrections online on November $14^{\text {th }}, 2019$.

12. Attended an Agilent ICP-OES and MS webinar titled Picking the best ICP-MS Cones online on November $7^{\text {th }}, 2019$.

13. Attended and presented at SCIX conference on October $15^{\text {th }}-18^{\text {th }}$, 2019, in Palm Springs, CA.

14. Attended an Agilent ICP-OES and MS webinar titled Remote Instrument Control: ICP$M S$ online on October $10^{\text {th }}, 2019$.

15. Attended an Agilent ICP-OES and MS webinar titled Removal of Spectral Interferences online on September $26^{\text {th }}, 2019$.

16. Attended an Agilent ICP-OES and MS webinar titled Polyatomic Interference Correction online on September 12 ${ }^{\text {th }}, 2019$.

17. Attended the World Scout Jamboree on July $29^{\text {th }}-30^{\text {th }}$, 2019, in Mt. Hope, WV to collect hundreds of background and shooter samples for a large GSR population study.

18. Poster author and secondary mentor for the WVU Summer Undergraduate Research Experience mentee in July 2019.

19. Attended and presented at the Mid-Atlantic Association of Forensic Science (MAAFS) conference on May $9^{\text {th }}-10^{\text {th }}, 2019$ in Morgantown, WV.

20. Completed an online course from Stanford University named Writing in the Sciences on May $17^{\text {th }}, 2019$.

21. Attended JMPro webinar titled Data Mining and Predictive Modeling online on March $3^{\text {rd }}, 2019$.

22. Attended JMPro webinar titled Building Dashboards to Access and Share Updated Analysis online on March 1 $1^{\text {st }}, 2019$.

23. Attended and presented at American Academy of Forensic Science (AAFS) on February $19^{\text {th }}-22^{\text {nd }}, 2019$, in Baltimore, MD.

24. Attended an Agilent webinar titled ICPGO-Fast, Easy, GO!- Elemental analysis made easy online on January $29^{\text {th }}, 2019$.

25. Attended the Forensics@NIST symposium on October $7^{\text {th }}-8^{\text {th }}, 2018$, in Gaithersburg, MD.

26. Trained by the Sacramento Country District Attorneys' Crime Laboratory (SCDACL) in the trace evidence department on the procedure for automated gunshot residue (GSR) 
detection and I, in turn, provided an overview of the rapid methods for GSR detection developed by our laboratory, which lead to further collaboration between our two laboratories (September $17^{\text {th }}-20^{\text {th }}, 2018$, in Sacramento, CA).

27. Technical training from Perkin Elmer for micro-FTIR instrument on June $20^{\text {th }}, 2018$.

28. Technical training on Applied Spectra J200 Tandem LIBS instrument on May 22-23 ${ }^{\text {rd }}$, 2018.

29. Attended the West Virginia University Workshop on Laser Ablation ICP-MS and LIBS Confirmation on May $22^{\text {nd }}-23^{\text {rd }}, 2018$ in Morgantown, WV.

30. Attended the Chesapeake Bay Division- International Association of Identification conference on April $26^{\text {th }}-27^{\text {th }}, 2018$, in Morgantown, WV.

31. Technical training on Agilent 7800 ICP-MS instrument on March $7^{\text {th }}-9^{\text {th }}, 2018$.

32. Attended Midwestern Association of Forensic Science (MAFS)/ American Society of Trace Evidence Examiners (ASTEE) conference on September 19 1th $^{\text {th }} 2^{\text {nd }}, 2017$ in Columbus, $\mathrm{OH}$.

33. Assisted Dr. Tatiana Trejos with the research preparation and organization for the Organization of Scientific Area Committees for Forensic Science for Trace Evidence.

34. Lead female counselor for the two-week WVU Next-Generation Forensic Science Camp on June $12^{\text {th }}-24^{\text {th }}, 2017$.

35. Attended the Chesapeake Bay Division- International Association of Identification conference on April 21 $1^{\text {st }}-22^{\text {nd }}, 2017$, in Fredrick, MD.

36. Attended the Law Symposium on Flawed Forensic Science and Innocence on March $4^{\text {th }}$, 2017 to understand forensic science from the point of view of the criminal justice system.

37. Project team leader to design and create a mock crime scene using the Matterport 3D camera system in collaboration with the journalism department at WVU.

38. Attendee of the Chesapeake Bay Division- International Association of Identification one-day conference on October 15, 2016, in Morgantown, WV.

39. Attended the Chesapeake Bay Division- International Association of Identification conference on April $22^{\text {nd }}-23^{\text {rd }}, 2016$, in Williamsburg, VA.

40. Trained in Latent Print Photography by Ken Bauer at a continuing education training for working professionals in the forensic and law enforcement field in March $21^{\text {st }}-23^{\text {rd }}, 2016$. 